\title{
NUMERICAL MODELING OF DYNAMIC COMPACTION IN COHESIVE SOILS
}

\author{
A Dissertation \\ Presented to \\ The Graduate Faculty of The University of Akron \\ In Partial Fulfillment \\ of the Requirements for the Degree \\ Doctor of Philosophy
}

Khaled Mostafa

December, 2010 


\title{
NUMERICAL MODELING OF DYNAMIC COMPACTION IN COHESIVE SOILS
}

\author{
Khaled Mostafa
}

Dissertation

Approved:

Accepted:

Advisor

Dr. Robert Liang

Committee Member

Dr. Ping Yi

Committee Member

Dr. Ala Abbas

Committee Member

Dr. Zhenhai Xia

Committee Member

Dr. Kevin Kreider
Department Chair

Dr. Wieslaw Binienda
Dean of the College

Dr. George K. Haritos

Dean of the Graduate School

Dr. George R. Newkome

Date 


\begin{abstract}
ABASTRACT
Dynamic Compaction (DC) has been used as soil improvement techniques for decades. The technique involves subsequent drops (tamps) of heavy weights onto the ground surface to densify the underlying soil. The technique is widely spreading because of its economical advantage and technical ease. DC is relatively cheap since no soil replacement or material addition is required. In addition, the equipment used for the process is simple. Therefore, shortly after the successful application of the DC on cohesion-less soils the technique has been extended for cohesion soils.

Usually, a crane is used to lift the tamper; Tamper weight ranges from 10 to 20 tons and drope height ranges from 10 to 20 meters. Then, the tamper is released to free fall and strike soil surface. The process is done in rounds. At each round, tamping takes place over a pre-defined grid. A decision for a second round of tamping is made when the depth of the induced crater is greater than the tamper height. A shifted grid is used for the second round of tamping after soil surface is bulldozed. In-situ tests (CPT or SPT) to estimate soil properties after compaction and to decide if further treatment is needed.

Empirical guidance on the zone of influence (e.g., the depth of improvement and the degree of improvement) as a function of impact energy has been summarized in the literature. Furthermore, there have been a limited number of numerical simulation techniques that were developed specifically for modeling the impact phenomenon of dynamic compaction on soil medium. However, the past numerical simulations were
\end{abstract} iii 
limited to the use of a simplified elastic perfectly plastic model to represent soils, which may not capture the highly plastic and nonlinear behavior of cohesive soils under dynamic compaction.

The work presented throughout this report is concerned with studying the dynamic compaction in cohesive soils. Two-dimension and three-dimension finite element models were developed. Modified Cam-Clay soil constitutive model, which captures the highly nonlinear behavior of soil, has been used throughout the presented work. A parametric study by mean of changing each Cam-Clay parameter at a time was conducted. Correlation between the soil properties and tamping energy per blow and the zone of influence is presented. In addition the amplitude of the wave velocity, which is propagating outward the compaction spot, is estimated as a function of tamping energy and Cam-Clay parameters. Utilizing the three dimensional model, the interaction between nearby drops was studied. Finally design charts for a step-by-step design methodology for DC in cohesive soils were presented. 


\section{ACKNOWLEGEMENTS}

I want to express my gratitude to a lot of people without their help I would not be able to finish this dissertation. I would like to thank Prof. Robert Y. Liang for all the support he has given me. He was so understanding and encouraging. He helped me for long hours in his office giving advice and recommendation to improve the work. I would like to thank him on the personal level for the friendly way he treats his research team. It has been pleasure working with him.

A special thanks for my family for their help during my course work as well as the thesis work. I really appreciate their understanding of how busy I used to get in the defense days. They were encouraging and helpful. Finally, I would like to thank all the University of Akron staff for all the support they gave me to finish this work. 


\section{TABLE OF CONTENSTS}

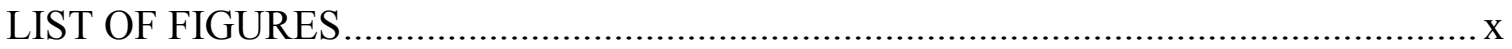

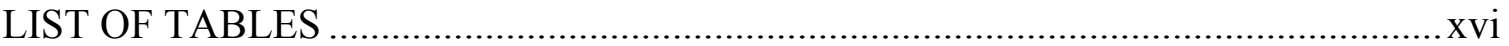

\section{CHAPTER}

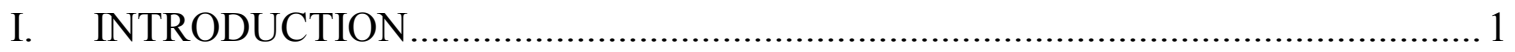

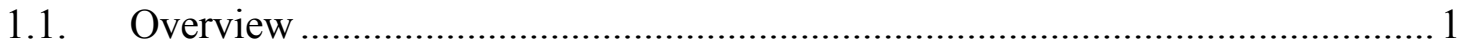

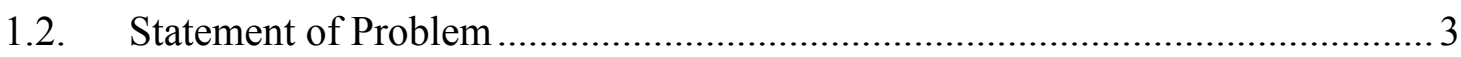

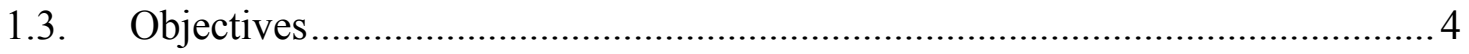

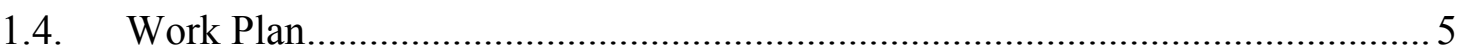

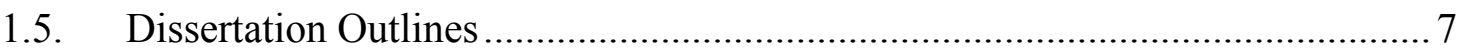

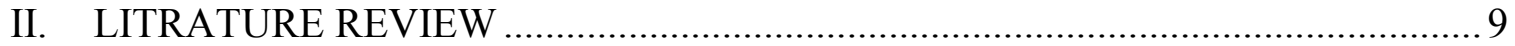

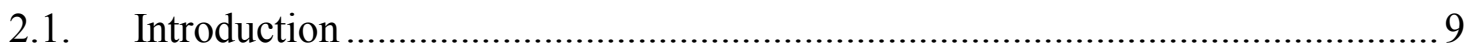

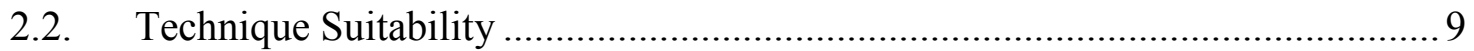

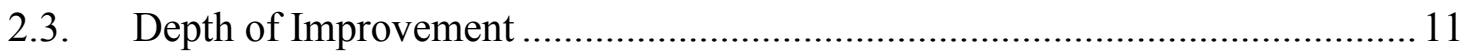

2.4. Lateral Extent of Improvement ………………......................................... 15

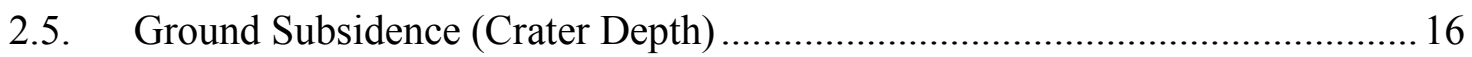

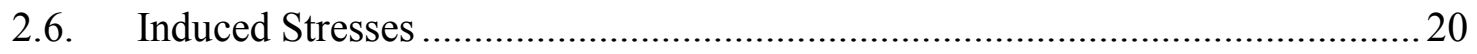

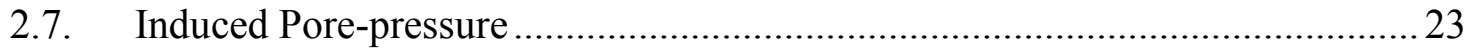




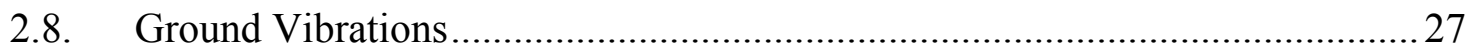

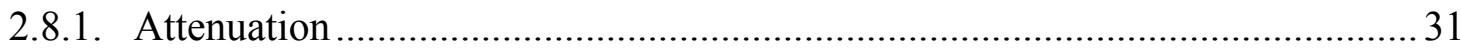

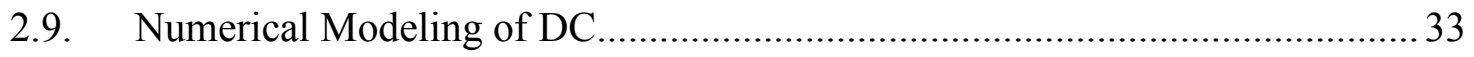

2.10. Soil Constitutive Models Used by Previous Finite Element Modeling ............. 38

III. DEVELOPMENT OF MODELING TECHNIQUES WITH VALIDATIONS ....... 41

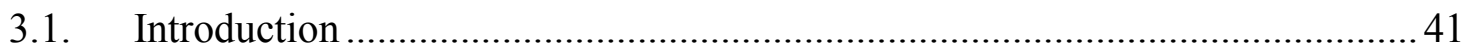

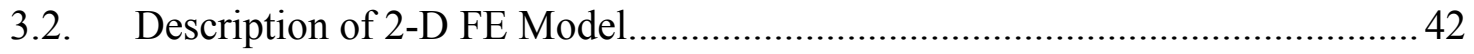

3.2.1. Constitutive Model ................................................................................. 43

3.2.2. Geostatic Step and Dynamic Integration Scheme ....................................... 44

3.2.3. Mesh Convergence and Model Dimensions............................................... 47

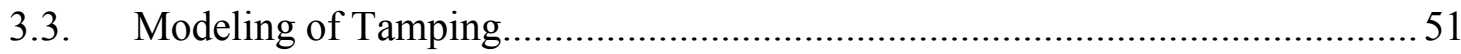

3.3.1. Tamping with Initial Velocity ........................................................... 51

3.3.2. Tamping with Force-time Function........................................................ 52

3.3.3. Improving Numerical Performance of Multiple Drops Model .......................55

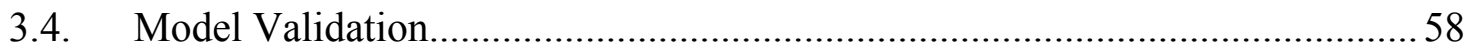

3.4.1. Validation of FE Model with Cam-Clay Constitutive Model ..........................59

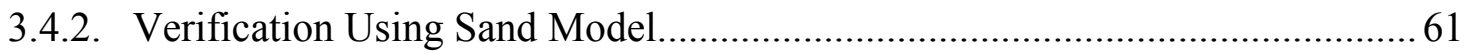

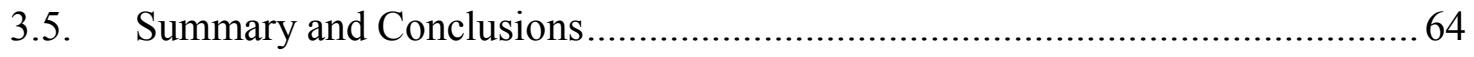

IV. MODELING RESULTS OF DYNAMIC COMPACTION IN COHESIVE SOILS 67

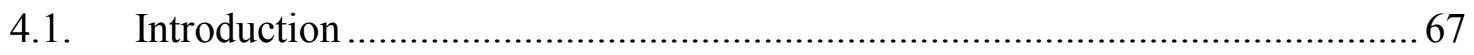

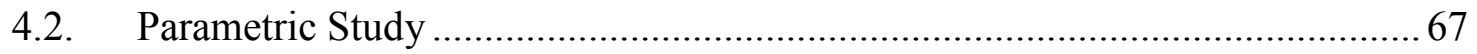

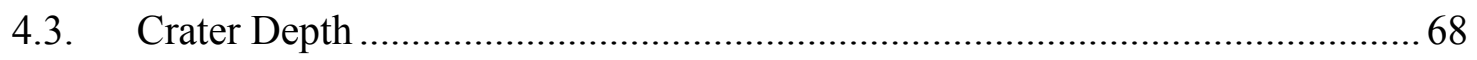




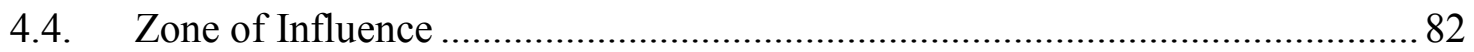

4.5. Peak Particle Velocity ............................................................................ 107

4.5.1. Frequencies Content of The Associated Waves ....................................... 123

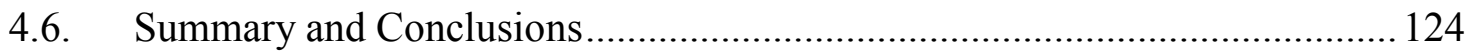

V. EFFECT OF MULTIPLE IMPACTS FROM ADJACENT IMPACT SPOTS ...... 126

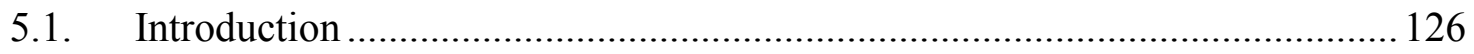

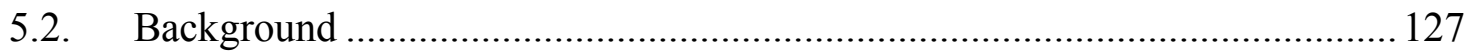

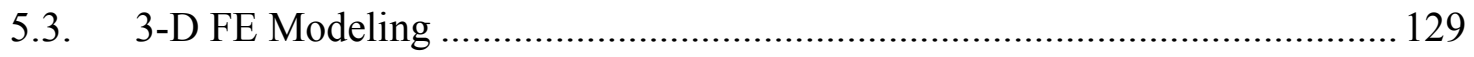

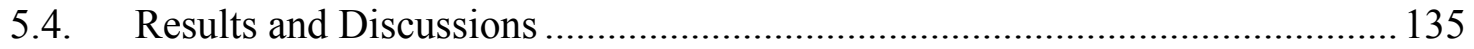

5.4.1. Results for Model of 3R Footprint Spacing $(\mathrm{R}=1.25 \mathrm{~m})$.......................... 136

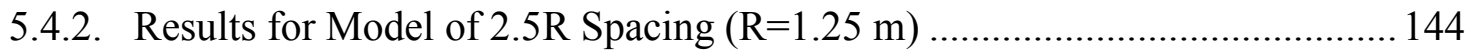

5.5. Conclusion and Summary ................................................................. 150

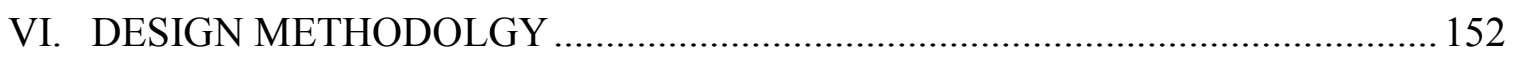

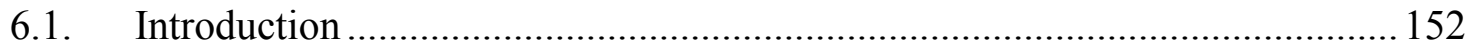

6.2. Crater Depth Effect on Void Ratio Distribution ......................................... 153

6.3. Classification of Soil Suitability for DC …............................................... 158

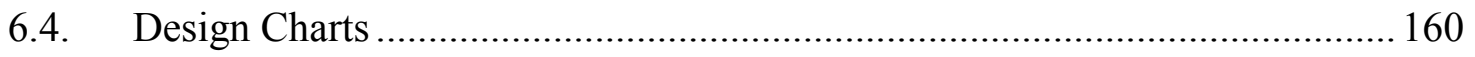

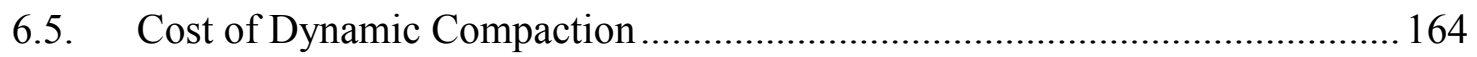

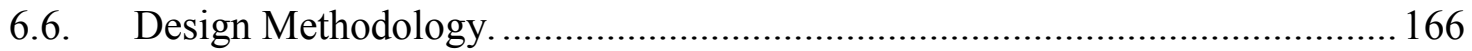

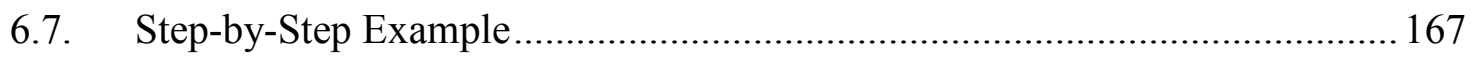

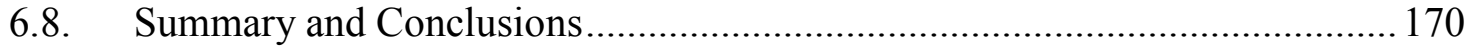

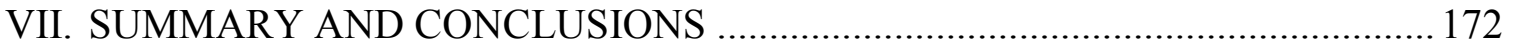

viii 


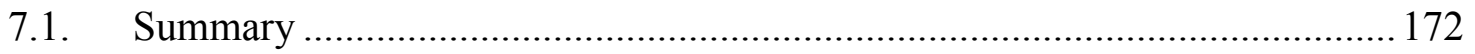

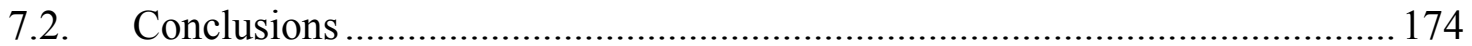

7.3. Recommendations for Future Studies .................................................. 177

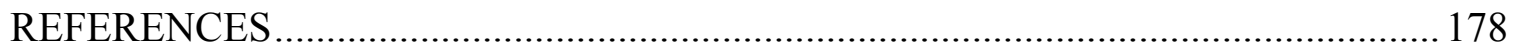




\section{LIST OF FIGURES}

Figure

Page

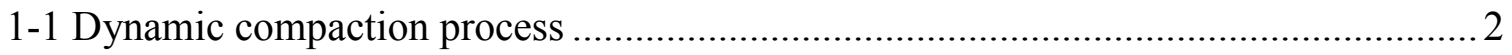

1-2 Flowchart depicting the general work plan..................................................... 7

2-1 Range of soil gradation of deposits suitable for DC (Lukas 1986).......................... 10

2-2 Maximum depth of influence versus drop energy (Mitchell 1981) .......................... 12

2-3 Maximum depth of influence versus drop energy (Mayne 1984)............................. 13

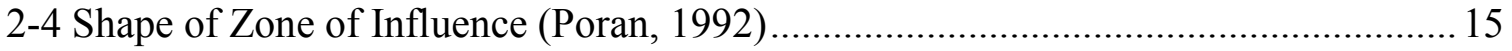

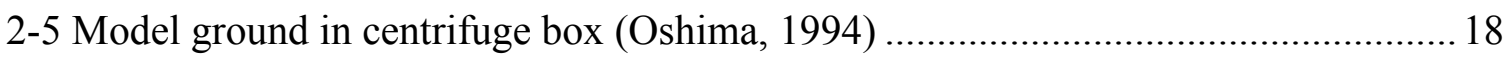

2-6 Effect of tamping energy on crater depth (Mayne et al. 1984) ................................ 19

2-7 Normalized crater depth for collapsible soils (Rollins and Kim 1994) .................... 19

2-8 Measured tamper (ram) acceleration (Oshima, 1994) ........................................... 20

2-9 Comparison between predicated and measured dynamic stresses (Mayne,1983) ...... 22

2-10 Comparison of predicted and measured pore pressure Gunaratne, 1996) ...............25

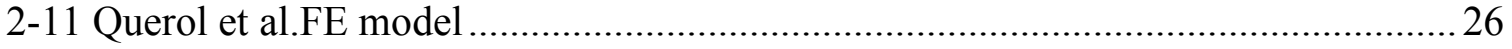

2-12 Waves generated during dynamic compaction, (Richart et al, 1970) ...................... 27

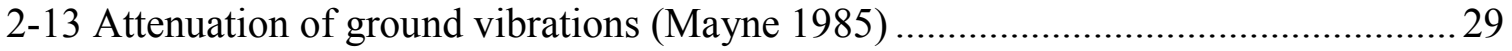

2-14 Attenuation of normalized PPV versus normalized distance (Mayne 1985) ............ 30

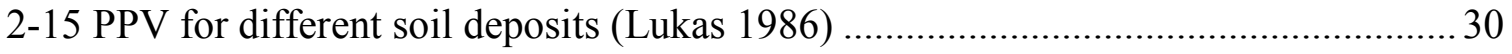

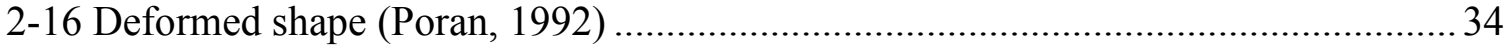




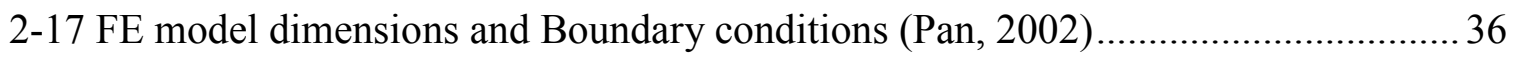

2-18 FE model dimensions and boundary conditions (Gu et al. 2002)........................... 37

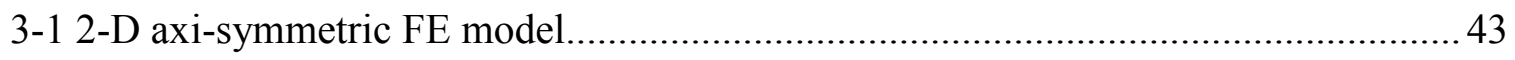

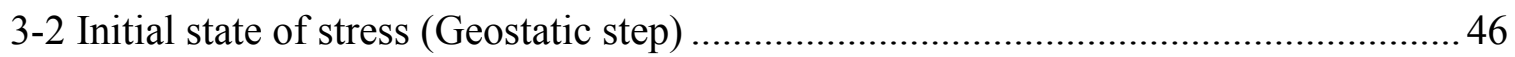

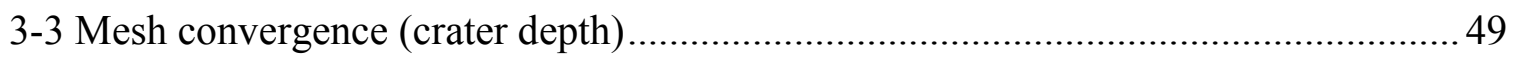

3-4 Mesh convergence (void ratio distribution beneath the tamper) ............................50

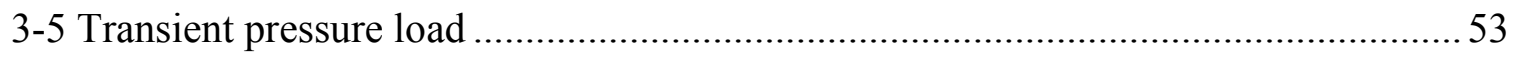

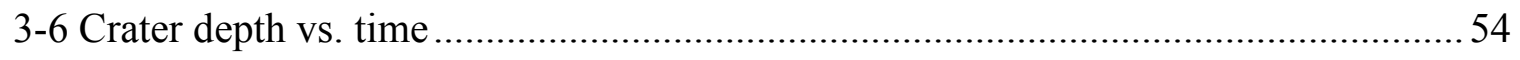

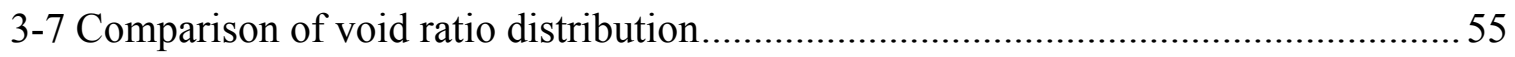

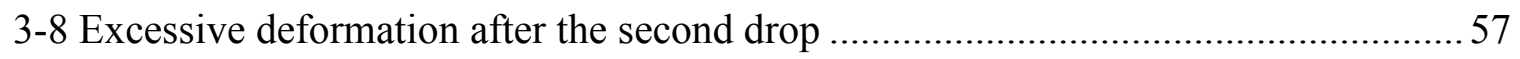

3-9 Groove location and element distribution for the proposed solution........................57

3-10 Void ratio distribution for improved numerical performance (drops 1 to 3 )............58

3-11 Void ratio distribution for improved numerical performance (drops 3 to 6 )............58

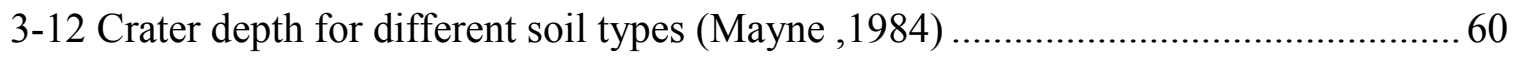

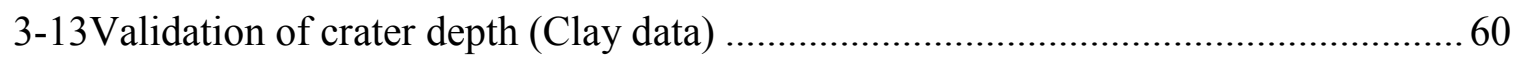

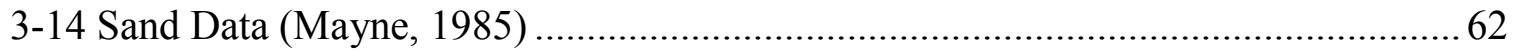

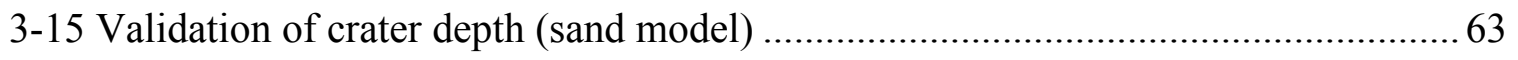

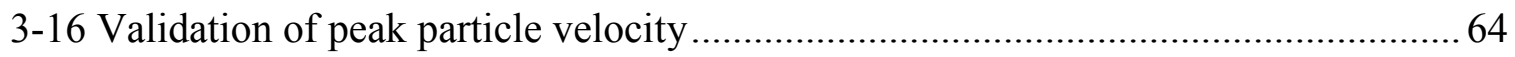

4-1 Variation of crater depth with tamper velocity …........................................ 70

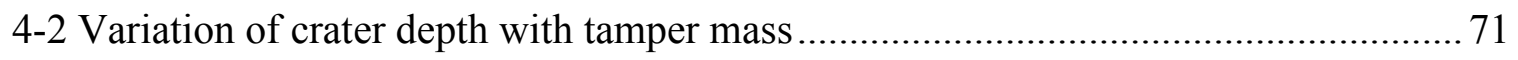

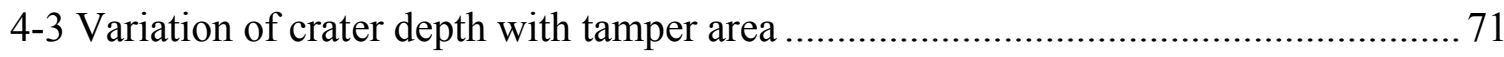

4-4 Effect of multiple drops on crater depth ..................................................... 72

4-5 Effect of tamper momentum on crater depth due to single drop ............................. 73 
4-6 Variation of crater depth with initial soil density 75

4-7 Variation of crater depth with pre-consolidation pressure..................................... 76

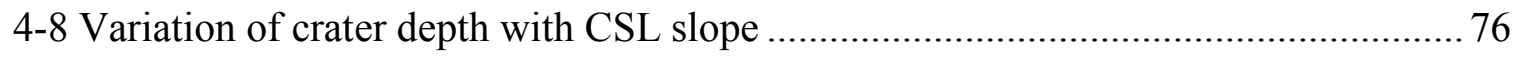

4-9 Variation of crater depth with logarithmic plastic bulk modulus $(\lambda) \ldots \ldots \ldots \ldots \ldots \ldots \ldots . . . . . . . . . . . .77$

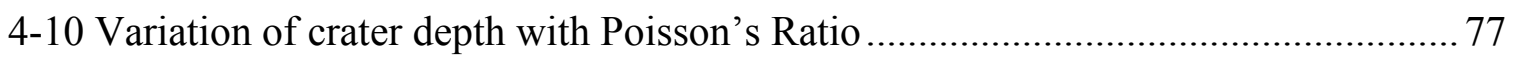

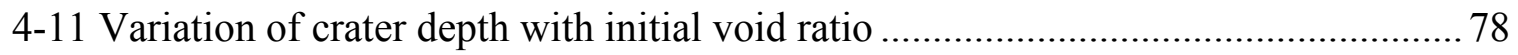

4-12 Relative change in crater depth vs. relative chance in soil properties .................... 79

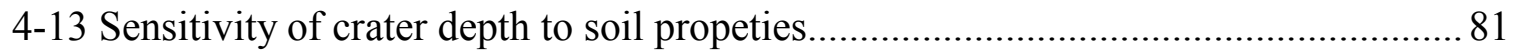

4-14 Comparison of the predicted crater depth with crater depth................................ 81

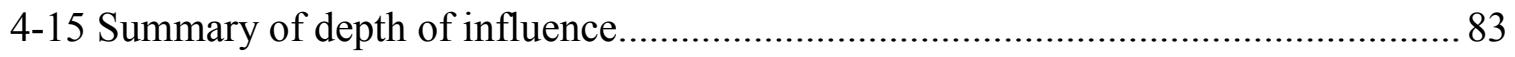

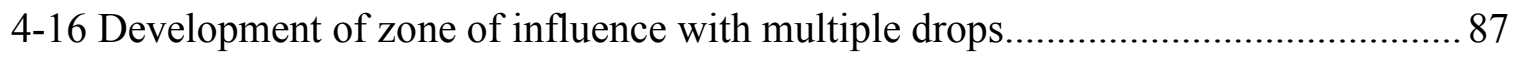

4-17 Variation of void ratio distribution with tamper mass ....................................... 88

4-18 Variation of void ratio distribution with drop height ........................................ 89

4-19 Variation of void ratio distribution with tamper radius ..................................... 90

4-20 Void ratio reduction variations with normalized energy per blow ......................... 91

4-21 Void ratio reduction variations with number of drops ...................................... 91

4-22 Variation of void ratio distribution with initial soil density ................................ 95

4-23 Variation of void ratio distribution with pre-consolidation pressure.......................96

4-24 Variation of void ratio distribution with CSL slope (M) .................................... 97

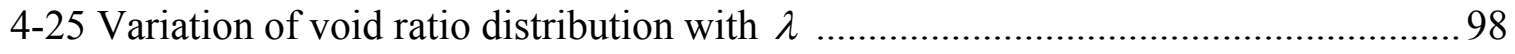

4-26 Variation of void ratio distribution with Poisson's ratio ...................................... 99

4-27 Variation of void ratio distribution with the relative change of void ratio ............. 100

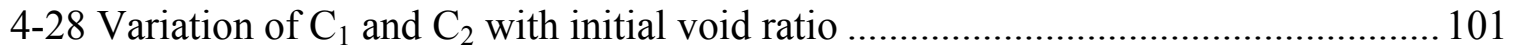




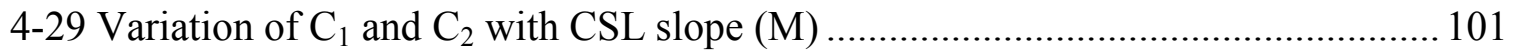

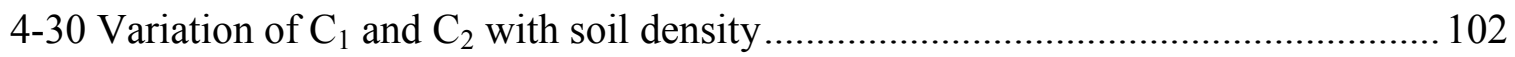

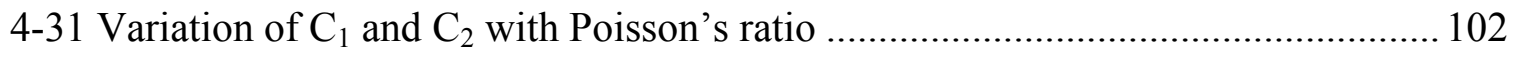

4-32 Variation of $\mathrm{C}_{1}$ and $\mathrm{C}_{2}$ with logarithmic plasticity bulk modulus $(\lambda) \ldots \ldots \ldots \ldots \ldots \ldots . . .103$

4-33 Variation of $C_{1}$ and $C_{2}$ with pre-consolidation pressure …………………......... 103

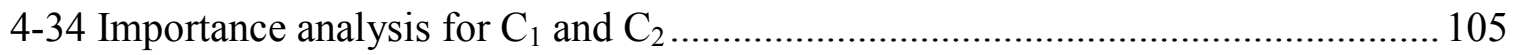

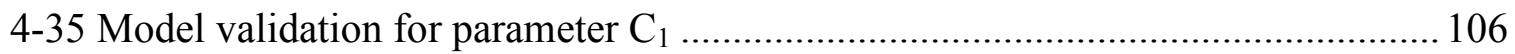

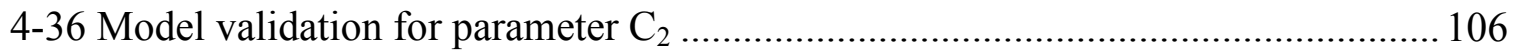

4-37 Variation of PPV with distance for different tamper mass ......................................112

4-38 Variation of PPV with distance for different drop height ...................................... 113

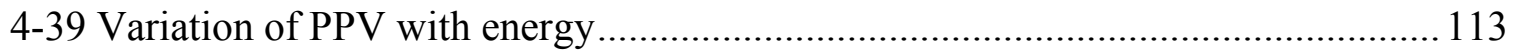

4-40 Variation of PPV with distance for different initial void ratio ..............................114

4-41 Variation of PPV with distance for different initial soil density ........................... 114

4-42 Variation of PPV with distance for different pre-consolidation pressure................ 115

4-43 Variation of PPV with distance for different $\lambda$ values ........................................115

4-44 Variation of PPV with distance for different Poisson's ratio ....................................116

4-45 Variation of PPV with distance for different CSL slope (M) ...............................116

4-46 Variation of PPV with soil properties (FE results) ...............................................117

4-47 Variation of PPV with soil properties (both Fe and Mayne model) .......................117

4-48 Variation of $\mathrm{B}_{1}$ and $\mathrm{B}_{2}$ with initial void ratio ..................................................118

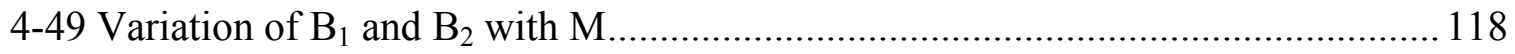

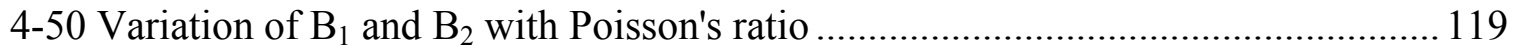

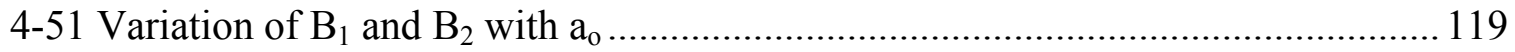


4-52 Variation of $\mathrm{B}_{1}$ and $\mathrm{B}_{2}$ with $\lambda$

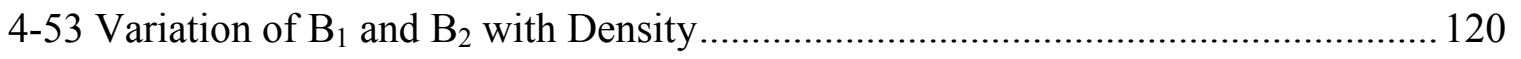

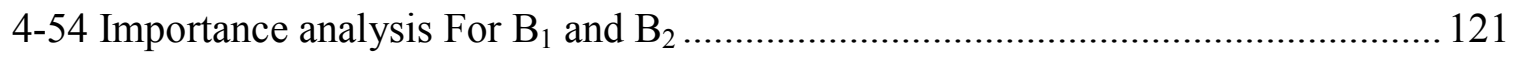

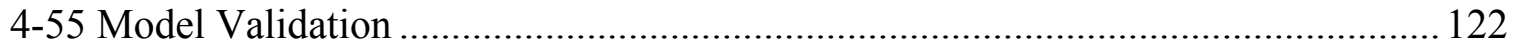

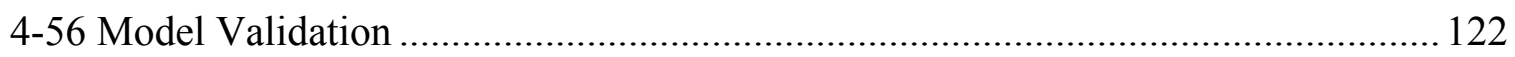

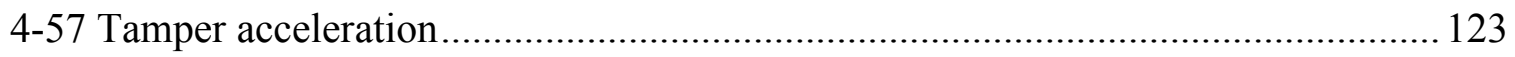

4-58 Frequency content of the tamper acceleration .................................................... 124

5-1 FE mesh for dynamic compaction modeling ...................................................... 130

5-2 Proposed groove

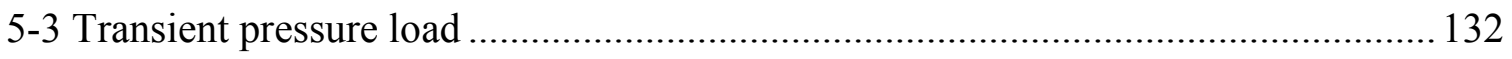

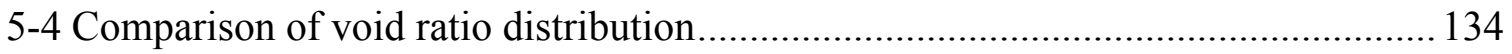

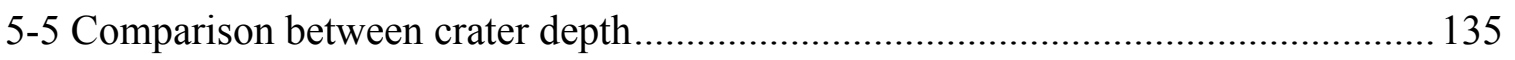

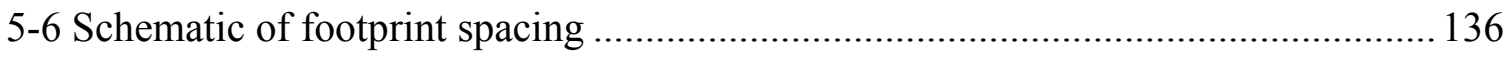

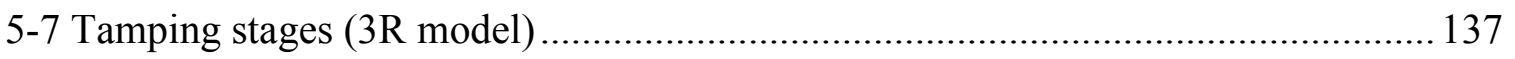

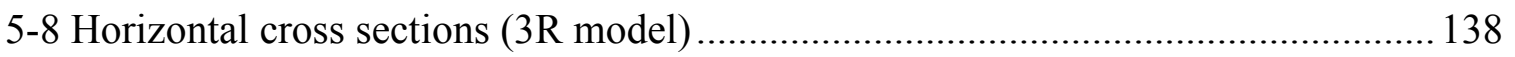

5-9 Interaction between the nearby tamping along footprint side (3R model) ............... 139

5-10 Interaction between the nearby tamping along footprint diagonal (3R model) ...... 140

5-11 Void ratio distribution beneath point A after tamping at each spot (3R model)..... 142

5-12 Void ratio distribution beneath point B after tamping at each spot (3R model).... 143

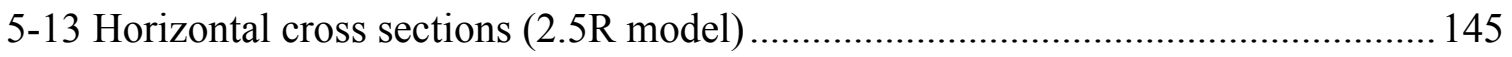

5-14 Interaction between the nearby tamping along footprint side (2.5R model) .......... 146

5-15 Interaction between the nearby tamping along footprint diagonal (2.5R model) ... 147

5-16 Void ratio distribution beneath point A after tamping at each spot (2.5R model).. 148 xiv 
5-17 Void ratio distribution beneath point B after tamping at each spot (2.5R model).. 149

6-1 Void ratio as a function of crater depth 158

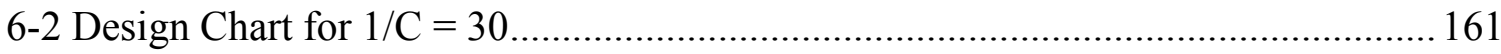

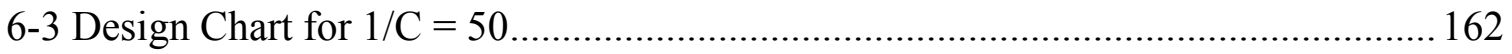

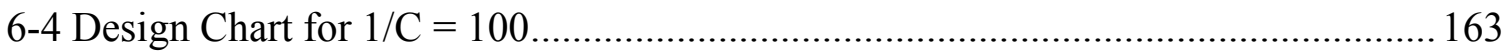

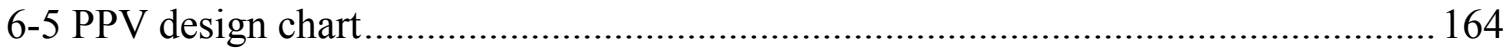

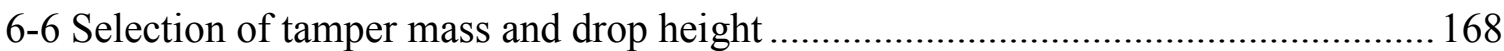

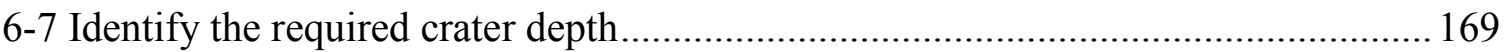




\section{LIST OF TABLES}

Table

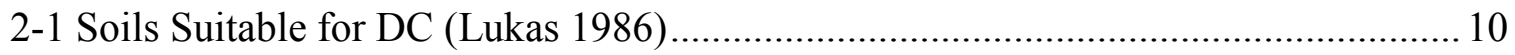

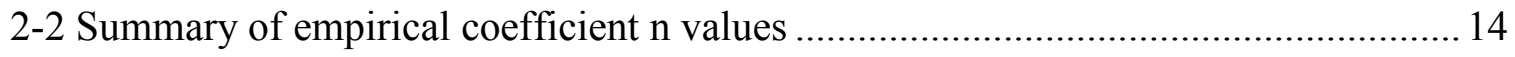

2-3 Surface subsidence for different deposits (Solocombe 1993).................................. 17

3-1 Baseline properties of Cam-Clay parameters .................................................. 44

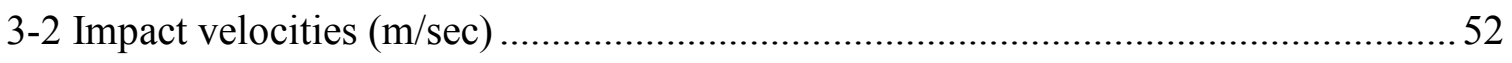

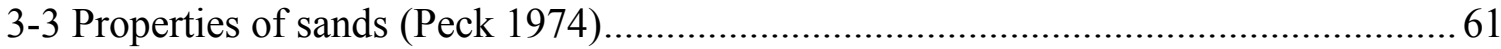

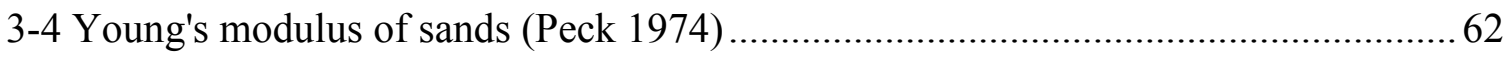

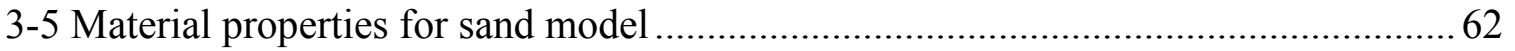

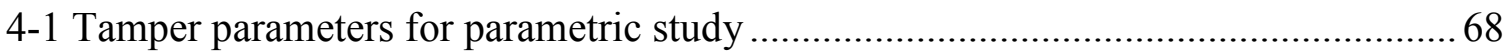

4-2 Cam-Clay model parameters for FE parametric study ........................................ 68

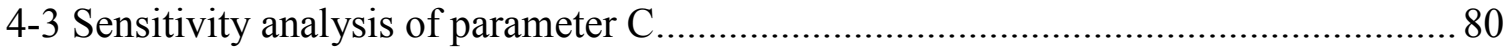

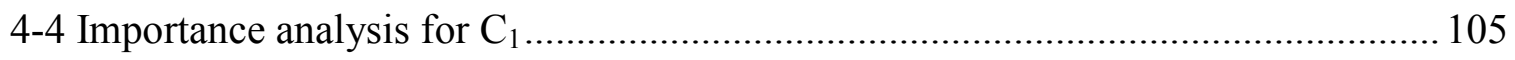

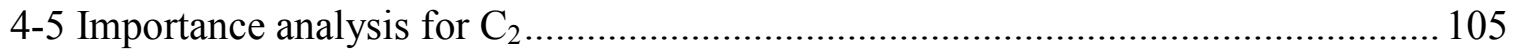

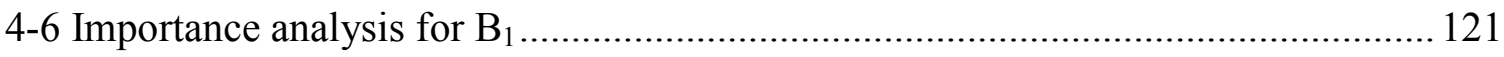

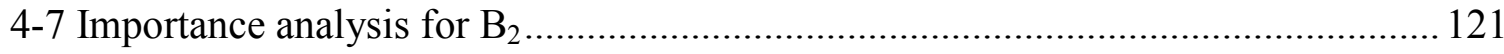

5-1 Cam-Clay model parameters for the 3-D model................................................ 131

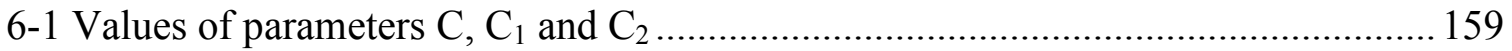




$$
\begin{aligned}
& \text { Notation } \\
& \text { W Tamper weight } \\
& m \quad \text { Tamper mass } \\
& H \quad \text { Drop height } \\
& n \quad \text { Constant } \\
& N \quad \text { Total number of blows } \\
& \text { A Contact Area } \\
& R \quad \text { Radius } \\
& P \quad \text { Crater depth } \\
& V_{o} \quad \text { Impact velocity } \\
& \text { E Impact energy } \\
& \text { I Impact energy Intensity } \\
& S \quad \text { Compaction Grid Spacing } \\
& \psi \quad \text { Dilation angle } \\
& v \quad \text { Poisson's ratio } \\
& \text { e Void ratio } \\
& k \quad \text { Permeability } \\
& V_{p} \quad \text { Pressure wave velocity } \\
& V_{s} \quad \text { Shear wave velocity } \\
& V_{R} \quad \text { Raleigh wave velocity }
\end{aligned}
$$


$g \quad$ Element size

$\lambda \quad$ Wave length

$f \quad$ Frequency

$q_{c} \quad$ Cone penetration resistance

$\kappa \quad$ Logarithmic bulk modulus

$\lambda \quad$ Logarithmic plasticity bulk modulus

$a_{0} \quad$ Initial yield surface size

M Stress ratio

Abbreviations

CD Dynamic Compaction

PI Plasticity Index

DI Depth of Influence

PPV Peak Particle Velocity

SPT Standard Penetration Test

CPT Cone Penetration Test

FEM Finite Element Method 


\section{CHAPTER I}

\section{INTRODUCTION}

\subsection{Overview}

Soil improvement has become a necessary process for most of the engineering projects due to the over-population in urban areas and the need to utilize landscapes with soil properties that are not ideal for construction. It is also said that sites with optimal soil conditions are precious nowadays. While soil improvement is known since ancient times, its objective stills remain the same. Soil Improvement aims to enhance soil properties to reduce the construction and foundation cost, save project time, and/or reduce any unexpected risks.

Soil improvement techniques fall under several categories. Improvement can take place by densification, adhesion, reinforcement, removal and replacement, biological, and physico-chemical alterations. Each category has its specific application in which it is more competitive than the other categories. Densification in general is the most commonly used because it is relatively cheaper than other techniques especially when large area of construction is to be improved. Densification usually takes place by mean of soil tamping. In addition, wick drains may be used in low permeability soils (e.g., clays) to fasten the dissipation of water pressure and increase the degree of improvement. 
Dynamic Compaction (DC) is one of the densification techniques in which soil is tamped heavily using a large mass (10-20 tons) dropped from a pre-determined height (10-20 meters). Tamping takes place over a grid of spots, as illustrated in Figure 1-1. Several tamping rounds are performed until the degree of improvement is achieved. The technique has been widely used due to: (1) successfully applied to different types of soils; (2) easy to implement (no special equipment or technology). (3) economically competitive (no material added or replaced); (4) environmentally safe (no chemicals injected or added).

There have been numerous documentations in the literature regarding the successful application of dynamic compaction in non-cohesive soils. Extensive theoretical, experimental, and in-situ tests were used for the determination of the dimensions of the zone of the influence and the associated peak particle velocity amplitude. Recently, DC has been extended to cohesive soils. The mechanisms of DC on cohesive soils will be the subject of interest in this study.

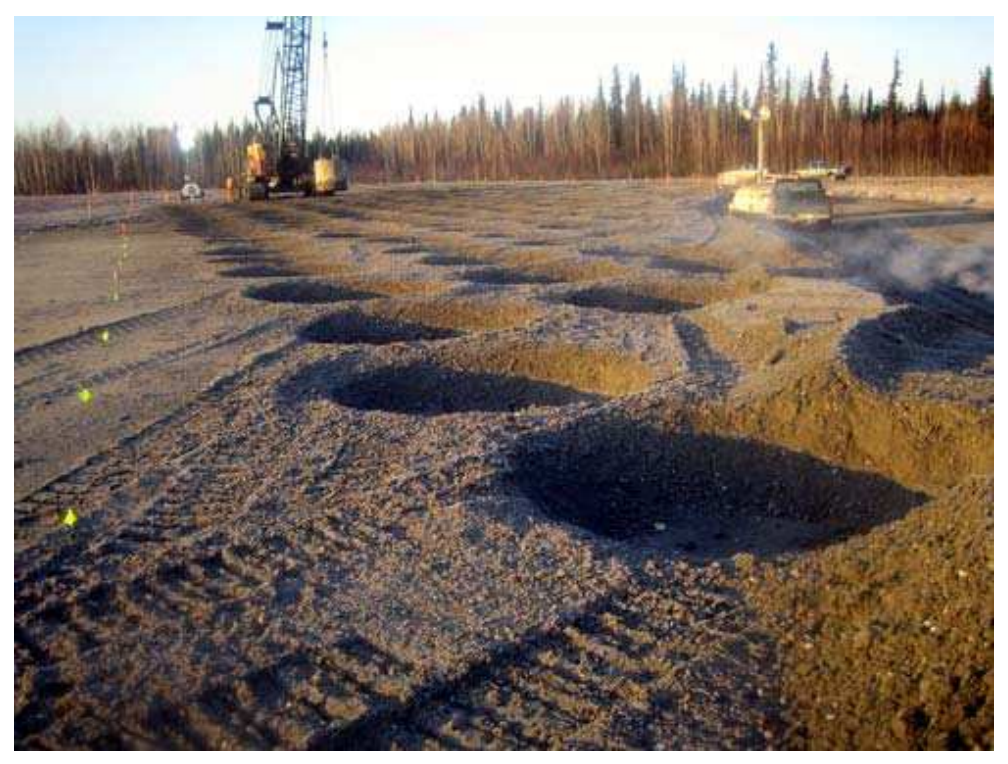

Figure 1-1 Dynamic compaction process 


\subsection{Statement of Problem}

The success of DC operation depends on the initial state of soil condition as well as the design methodology used to select the appropriate tamping energy per blow. The following should be available for the design engineers before starting a DC operation: (1) initial soil properties; (2) soil profile; (3) target depth of improvement and the maximum possible improvement. Based on the gathered information and the available design models, engineers may be able to: (1) select tamper mass; (2) select the appropriate drop heights; (3) select a number of drops to be performed at each spot; (4) identify the grid spacing; (5) consider for the hazards that may arise due to ground vibrations on any surrounding structure.

Several design models have been presented in the literature, which were developed primarily based on several in situ experiences and small scale laboratory tests performed on different types of soils. The current design models relate the maximum depth of influence to the tamping energy per blow by way of the equation $D I=n \sqrt{W H}$, where $n$ is a multiplier depending on soil properties and site condition. The maximum depth of improvement could be estimated using the available methods. However, those models do not present enough information to answer the following questions, especially for the cohesive soils: (1) what is the influence of soil properties on the depth and degree of improvement?; (2) what is the influence of soil properties on the amplitude of ground vibrations?; (3) what is the recommended grid spacing?; (4) what is the soil density after treatment? For these reasons, there is a need to study DC in cohesive soils to develop a complete design methodology that can identify the following: (1) induced crater depth;

(2) depth of improvement and density after treatment; (3) lateral extent of improvement 
at each tamping spot; (4) the associated ground vibration amplitude and the expected frequencies; (5) the optimum grid spacing.

\subsection{Objectives}

The objectives of this research are as follows.

- Develop a numerical modeling technique of Dynamic Compaction (DC), using finite element code ABAQUS, in cohesive soils represented by Cam-Clay constitutive model in order to achieve a better understanding of the behavior of cohesive soils under dynamic compaction.

- Perform sensitivity and parametric analysis to shed insights on the effects of the Cam-Clay constitutive model parameters on the maximum depth of influence and the degree of improvement in void ratio or density.

- Investigate through FE simulation the influence of Cam-Clay constitutive model parameters on the peak particle velocity (PPV) of the waves associated with dynamic compaction process.

- Develop a method to correlate soil crater depth, induced during DC operation, with the depth of influence to facilitate DC design and in situ monitoring.

- Develop a three dimensional finite element modeling technique of dynamic compaction in cohesive soils using ABAQUS to understand the group effect of the consecutive nearby tamping.

- Develop a criterion that can be used to estimate the optimum grid spacing for DC operation in cohesive soils. 
- Develop a complete design methodology for dynamic compaction in cohesive soils

\subsection{Work Plan}

From the above discussion, it is obvious that the current design models for DC in cohesive soils would not be sufficient to estimate the depth of improvement as well as the effective depth of improvement in cohesive soils. It can be noticed also that the current design models are too simplified to consider for variation in soil properties. In addition, the lateral extent of improvement, based on which the grid spacing is identified, has not been studied carefully in cohesive soils.

In order to achieve the proposed objectives, Finite Element Method (FEM) has been used to model the dynamic compaction in cohesive soils. The model needs to account for reduction in void ratio as well as the highly nonlinear behavior of soil during DC. Therefore, Cam-Clay constitutive model is used which is able to model the change in void ratio due to increase in effective stress applied to the soil. In addition, depth of improvement is influenced by the existing state of stress in the soil. A full state of stress distribution prior to commencing tamping action is modeled using Geostatic analysis step in ABAQUS.

Despite the benefits of densification, dynamic compaction is a process that could jeopardize the surrounding structures due to the accompanied ground vibrations. The severity of this influence is affected by the tamping energy per blow and the soil properties. A distinctive contribution of FE model is to account for the wave propagation during DC. Since very small elements of finite element mesh could result in numerical 
instability, while large elements do not allow the travel of short wave lengths; a special consideration should be made to assure the accurate modeling of wave propagation using FE simulations by select the appropriate element size and model dimensions.

The two-dimensional axi-symmetrical model is limited to simulate the tamping at one spot by taking advantage of the circular shape of impact mass. Therefore, a threedimensional model needs to be developed to simulate the consecutive tamping which is placed over a pre-defined nearby spots. The same features of two-dimensional model has been used for the three dimensional model (e.g., Cam-Clay model and initial state of stress). To simulate the real situation, four spots of tamping need to be tamped five times each. The lateral extent of improvement and interaction between tamping at nearby spots will be studied. The 3-D FE model contributes to the development of a design methodology for DC by identifying the optimum grid spacing.

Figure 1-2 illustrates the general work plan to achieve the objectives discussed in this section. Detailed description of each subdivision in this work plan is explained herein. 


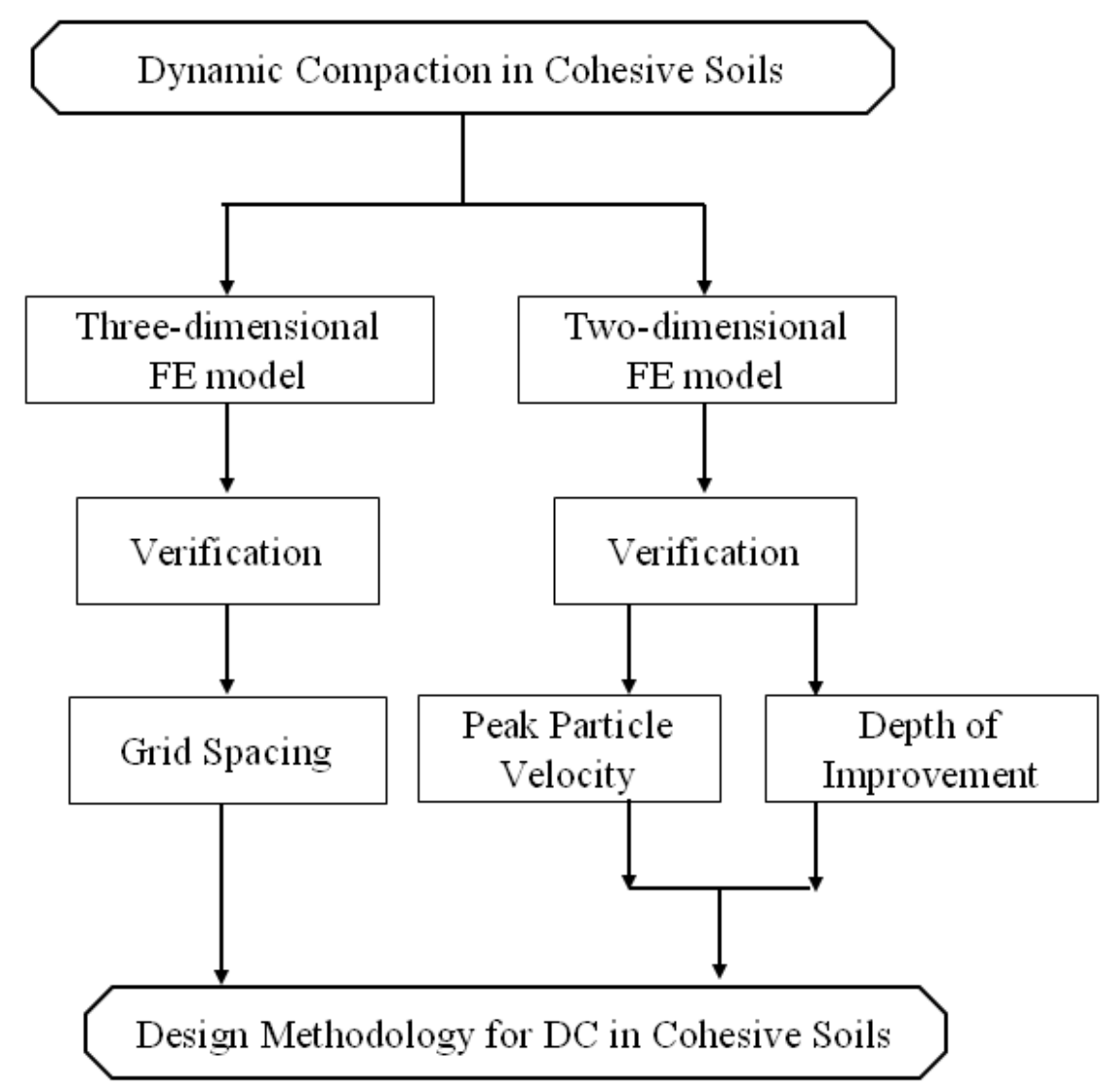

Figure 1-2 Flowchart depicting the general work plan

\subsection{Dissertation Outlines}

Chapter II provides a review of the work done by previous researchers on the subject relevant to dynamic compaction modeling, analysis, and design. The necessity to correlate the operational parameters of DC to initial and resulting soil properties was the motive to conduct the work presented in this dissertation.

Chapter III presents the numerical modeling techniques for dynamic compaction. Two modeling techniques were presented for single and multiple drops. The models were validated using field measurements. 
Chapter IV presents analysis of the simulation results from chapter III to estimate carter depth, density profile, and the Peak Particle Velocity (PPV) as a function of soil properties and tamping energy. The frequency content of the traveling wave was also studied.

Chapter V presents the three dimensional modeling of DC using ABAQUS. The group effect of subsequent tamping in adjacent tamping spots was studied. The effect of interaction between the nearby tamping on the distribution of void ratio was studied. Based on this interaction, a method was presented to estimate the optimum grid spacing for DC operation.

Based on the correlations obtained in chapters IV and V, a design methodology for DC in cohesive soils is presented in chapter VI. In addition, a simple in-situ test is proposed to identify applicability of certain site to DC.

The summary of work done, conclusions, and recommendations for future work are presented in Chapter VII. 


\section{CHAPTER II}

\section{LITRATURE REVIEW}

\subsection{Introduction}

A review of the pertinent literature reveals that a wealth of research has been expended in the past decades on the investigation of Dynamic Compaction (DC) applicability for different soils as well as development of design guidelines. This chapter reviews the most relevant design models. Technique suitability for different soil types, depth of influence (DI), crater depth, induced pore-pressure and the accompanied waves are the main concern for researchers when designing for DC operation. This chapter provides a synthesis of the most recent research conducted for the analysis and design of a DC operation.

\subsection{Technique Suitability}

DC was proven to be successful for granular soil or fill soils. Many other deposits have been considered for DC treatment because the relatively cheap cost and effectiveness of the technique compared to other soil treatment techniques. Because of the excess pore pressure induced during DC, saturated clayey soils were considered to be not suitable for DC. Clayey soils with highly impervious characteristics do not allow the dissipation of excess pore-pressure, resulting in decrease in soil bearing capacity. Therefore, pore pressure dissipation is necessary when treating saturated soils. 
Liquefaction may occur during DC operation in saturated sandy deposits. Lukas (1986) categorized suitability for DC based on soils properties. As can be seen in Table 2-1 and

Figure 2-1, soil permeability and plasticity index (PI) control the suitability of soils for DC.

Table 2-1 Soils Suitable for DC (Lukas 1986)

\begin{tabular}{c|c|c}
\hline Soil Category & Soil properties & Suitability for DC \\
\hline Pervious Soil deposits & $\begin{array}{c}k>10^{-5} \mathrm{~m} / \mathrm{sec} \\
P I=0\end{array}$ & $\begin{array}{c}\text { Improvements are } \\
\text { Achievable }\end{array}$ \\
\hline Intermediate soil deposits & $\begin{array}{c}10^{-5}>\begin{array}{c}k \\
0\end{array} \\
0<P I<8\end{array}$ & $\begin{array}{c}\text { Dissipation of pore pressure } \\
\text { is a necessity }\end{array}$ \\
\hline Impervious clayey deposits & $\begin{array}{c}k<10^{-8} \mathrm{~m} / \mathrm{sec} \\
P I>8\end{array}$ & \begin{tabular}{c} 
Not Recommended \\
\hline
\end{tabular}
\end{tabular}

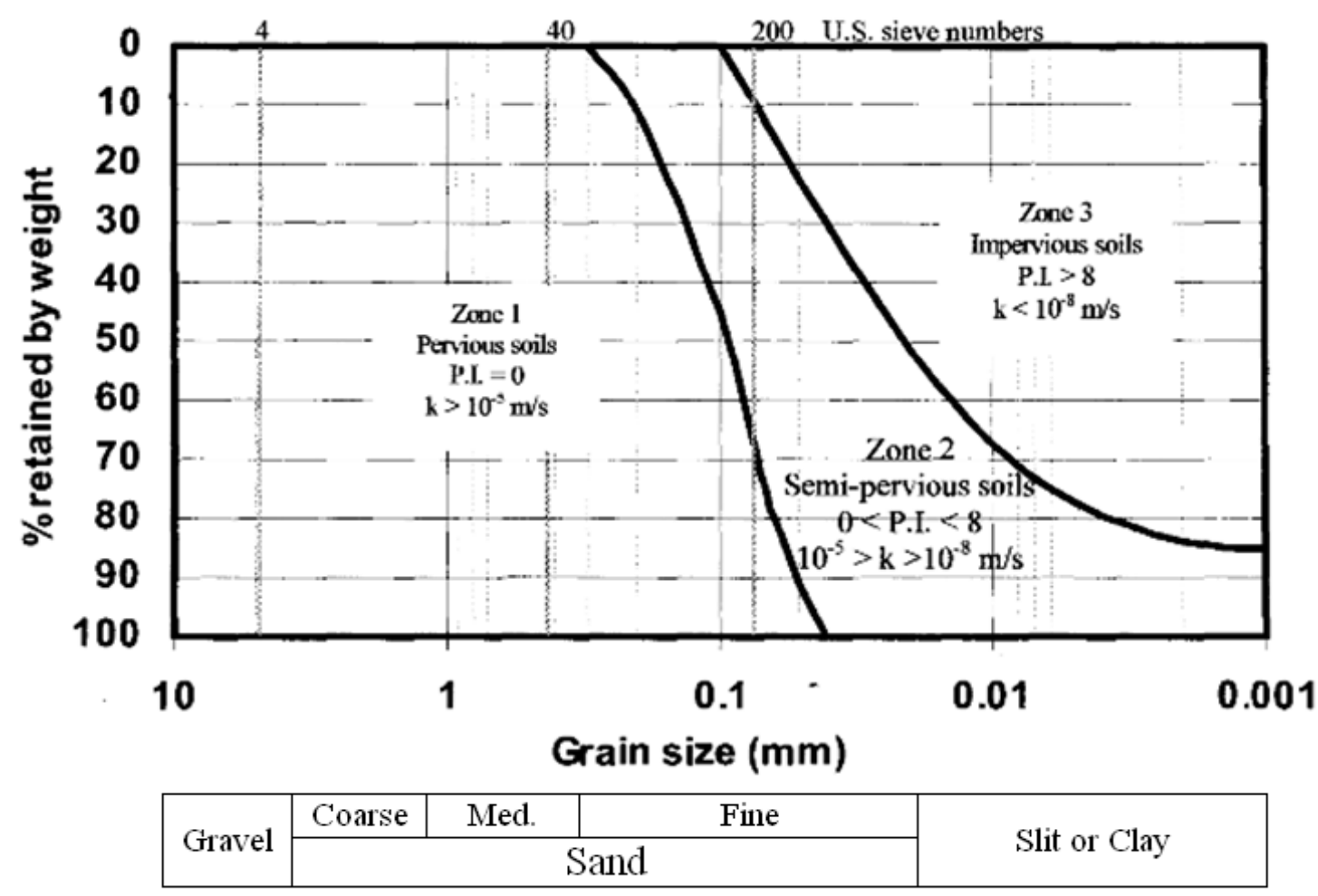

Figure 2-1 Range of soil gradation of deposits suitable for DC (Lukas 1986) 


\subsection{Depth of Improvement}

The effective Depth of improvement (DI) due to DC is generally considered as the depth measured from the crater bottom at which significant change in soil properties is noticed after compaction. A lot of research has been conduced to correlate the depth of improvement (DI) to the tamping energy per blow. The depth of maximum improvement, on the other hand, is the depth in which the maximum improvement of soil is achieved. In this section, the current design models to estimate the depth of improvement is reviewed and presented briefly. A summary of the experimental data from the literature is listed and presented in this section for future validation purposes.

Menard (1975) developed the compaction mechanism in saturated clays. The possibility of improving the mechanical properties of fine soils was discussed. The presence of micro-bubbles, the gradual liquefaction under repeated impacts, and the change of soil permeability due to fissure creation are the four points that was addressed in Menard's work about the mechanism of compaction. Increase in pore pressure is noticed after each impact which results in reduction in the bearing capacity of the soil. Subsequently, the compaction induced excess pore pressure dissipates with time. Menard proposed the following relationship to indicate the depth of improvement.

$$
D I=\sqrt{W H}
$$

where

$D I$ : Depth of improvement

$W$ : Tamper weight in tones

$H$ : Falling height in meters

Charles et al. (1981) studied the effectiveness of dynamic consolidation in improving the load carrying characteristics of the ground. Loose uncompacted fills was 
used for four sites whereas soft alluvial was used for the fifth one in their study. DC was effective for the loose soils. Depth of improvement could be measured and related to the total input energy. Preloading with surcharge combined with improved drainage was the recommended improvement technique for soft alluvial soil. Mitchell (1981) and Mayne (1984) summarized field measurements for considerable number of sites and generated charts representing energy versus depth of influences, as shown in Figure 2-2 and Figure 2-3. A multiplier $n$ was recommended to factor the square root of input energy for better data regression. There has been quite extensive research done in the past to identify the multiplier $n$ for different soils. Table 2-2 summarizes all the different values for $n$.

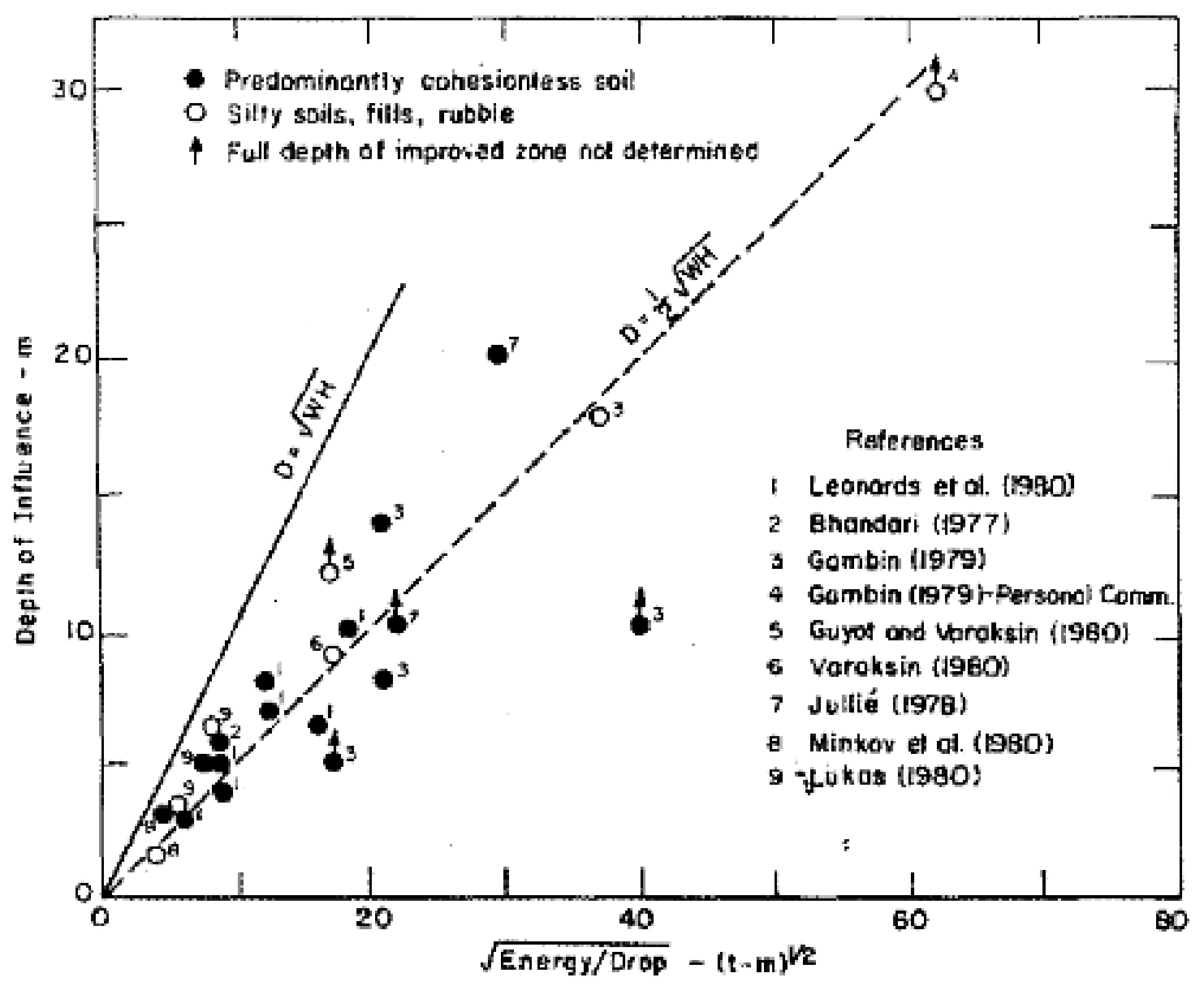

Figure 2-2 Maximum depth of influence versus drop energy (Mitchell 1981) 


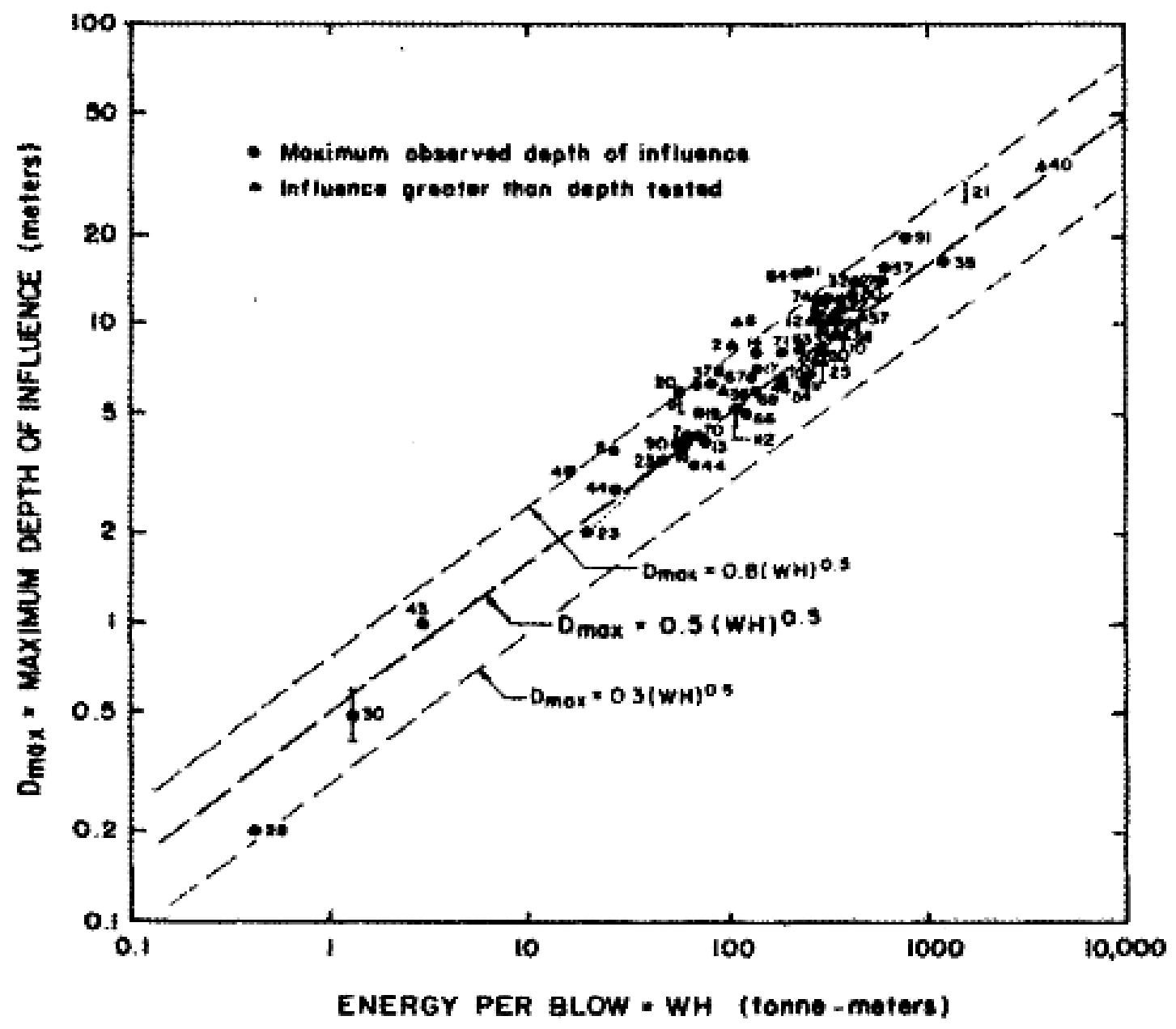

Figure 2-3 Maximum depth of influence versus drop energy (Mayne 1984) 
Table 2-2 Summary of empirical coefficient $n$ values

\begin{tabular}{l|c|l}
\hline Author & $n$ & Soil Type \\
\hline Menrad (1975) & 1 & \\
\hline Lonard (1980) & 0.5 & \\
\hline Bjolgerun & 1 & Rockfill \\
\hline \multirow{2}{*}{ Smoltcyk (1983) } & 0.5 & Soil with unstable structure \\
\cline { 2 - 3 } & 0.67 & Silts and sands \\
\cline { 2 - 3 } & 1 & Purely friction sands \\
\hline Lukas(1980) & $0.65-0.8$ & \\
\hline Mayne et al (1981) & $0.3-0.8$ & \\
\hline Gambin (1984) & $0.5-1.0$ & \\
\hline Qian & 0.65 & Fine sand \\
\cline { 2 - 3 } & 0.66 & Soft Clay \\
\cline { 2 - 3 } & 0.55 & Loess \\
\hline \multirow{2}{*}{ Van Impe } & 0.65 & Silty sand \\
\cline { 2 - 3 } & 0.5 & Clayey sand \\
\hline
\end{tabular}

Smith (1989) derived experimentally the relation between the depth of influence and tamper mass and striking velocity. The depth of influence is proportional to the maximum possible soil density. Dense soils show resistance to DC operations. Like Paul (1983), Smith model considers only one soil property to estimate DI.

$$
D I^{\prime}=\frac{m}{\rho_{\max } A}\left[-1+\left(\frac{\sigma_{e}+\alpha V_{o}^{2}}{\sigma_{e}}\right)^{1 / 2}\right]
$$

Where

$\rho_{\max }:$ Maximum possible soil density

$m:$ Falling mass

$A$ : contact area

$\sigma_{e}:$ Vertical stress at elasticity limit

$V_{o}$ : contact velocity

$\alpha$ : Density ratio 


\subsection{Lateral Extent of Improvement}

In an effort to investigate the vertical and lateral extent due to the DC, Poran (1992) derived a correlation between the plastic zone dimension, which is assumed to have a semi spheroid shape, and the input energy. The study was conducted experimentally on dry sands using a small scale specimen. Equations (2-3) and (2-4) show the dimensions of the plastic zone as a function of the normalized energy input.

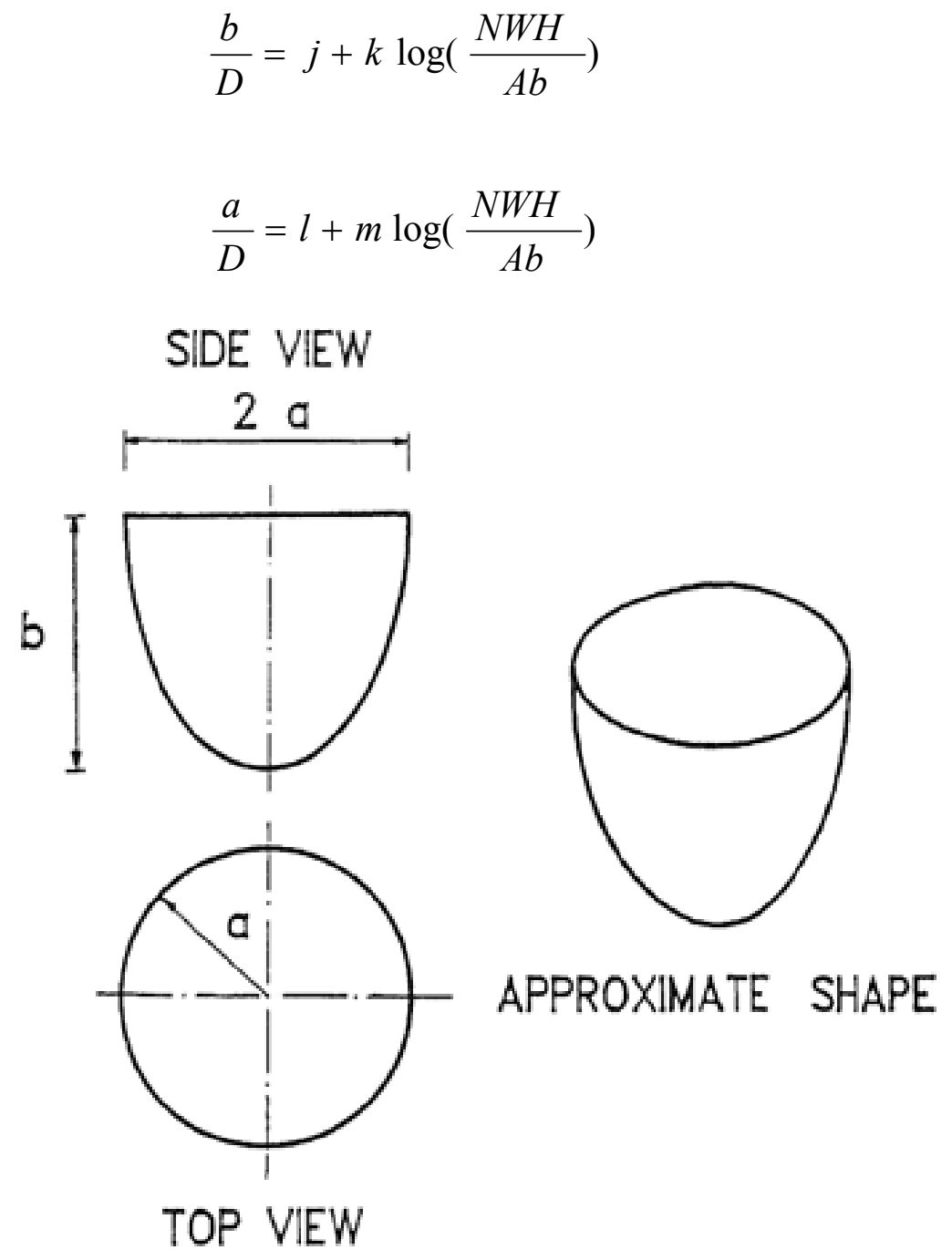

Figure 2-4 Shape of Zone of Influence (Poran, 1992) 
where

$a:$ Spheroid base radius

$b:$ Spheroid height

$D$ : Diameter of the falling mass

$N$ : Number of drops

$W:$ Falling weight

$H:$ Falling height

$A:$ Tamper area

$j, k, l, m:$ Regression constants

Chow (1994) investigated the lateral extent of DC operation at the ground surface by conducting cone penetration test (CPT) for a series of DC operations. Myerhof's correlation between the CPT results and friction angle was utilized. The change in friction angle is described by Equation (2-5). At distance three times the tamper diameter the effect of the DC is negligible. Chow also reported that, when compaction is placed over a spaced grid, the center point of the grid and middle of the grid sides are points of weakness for the process of dynamic compaction.

$$
\frac{\Delta \phi}{\Delta \phi_{b}}=0.642-1.180 \log \left(\frac{X}{D}\right)
$$

where

$\Delta \phi$ : Change in friction angle at distance $\mathrm{X}$ from the center $\Delta \phi_{b}$ : Change in friction angle beneath the tamper

$D$ : tamper diameter

$X$ : Distance measured from the center of tamper

\subsection{Ground Subsidence (Crater Depth)}

Crater results from the impact of the falling tamper onto the ground surface. Usually craters are surrounded by a heave of soil. The crater depth can be considered as a measure for the degree of improvement achieved. The typical value of crater depth ranges from 1.0 to 1.5 meter deep. Mayne (1984) prepared a chart, reproduced in Figure 2-6, to 
show the increase in crater depth with increasing impact energy. Solocombe (1993) used the percentage between the crater depths to the depth of influence as indication of soil suitability to DC operation as shown in Table 2-3. Rollins et al (1994) studied the change in crater depth with the number of drops. It can be concluded from Figure 2-6 that after number of drop, usually range from 6-8 drops, no more change in crater depth can be achieved using the same combination of tamper mass and the drop height.

Table 2-3 Surface subsidence for different deposits (Solocombe 1993)

\begin{tabular}{c|c|c}
\hline $\begin{array}{c}\text { Soil Category } \\
\text { (Figure (2-1)) }\end{array}$ & Soil Type & $\begin{array}{c}\text { Ground Subsidence } \\
\text { (\% of maximum depth of improvement) }\end{array}$ \\
\hline \multirow{2}{*}{ Zone 1} & Natural Sands & $3-10$ \\
\cline { 2 - 3 } & Granular fills & $5-15$ \\
\hline Zone 2 & Semi previous soils & N/A \\
\hline \multirow{2}{*}{ Zone 3} & Clay fills & $3-5$ \\
\cline { 2 - 3 } & Natural Clay & $1-3$ \\
\hline Other & Refuse and Peat & $7-20$ \\
\hline
\end{tabular}

Oshima (1994) studied analytically the crater depth resulting from the impact of falling weight. The crater depth could be related to the momentum of the falling weight as can be seen from Equation (2-6). The work was extended experimentally for the multiple drops compaction. A series of centrifuge model test of heavy tamping were performed. The model ground is a sandy soil compacted to relative $50 \%$ relative density and with a water content of $4 \%$ placed inside semi-cylinder which is $30(\mathrm{~cm})$ in diameter and $20(\mathrm{~cm})$ in height. The experimental setup is depicted in Model ground in centrifuge box (Oshima, 1994) Figure 2-5 Tamping takes place at centrifugal acceleration of $100 \mathrm{~g}$. ground deformation was observed through a glass plate on the front face. It was found that the crater depth is proportional to the square root of number of drops, Equations (2-6) and (2-7). 


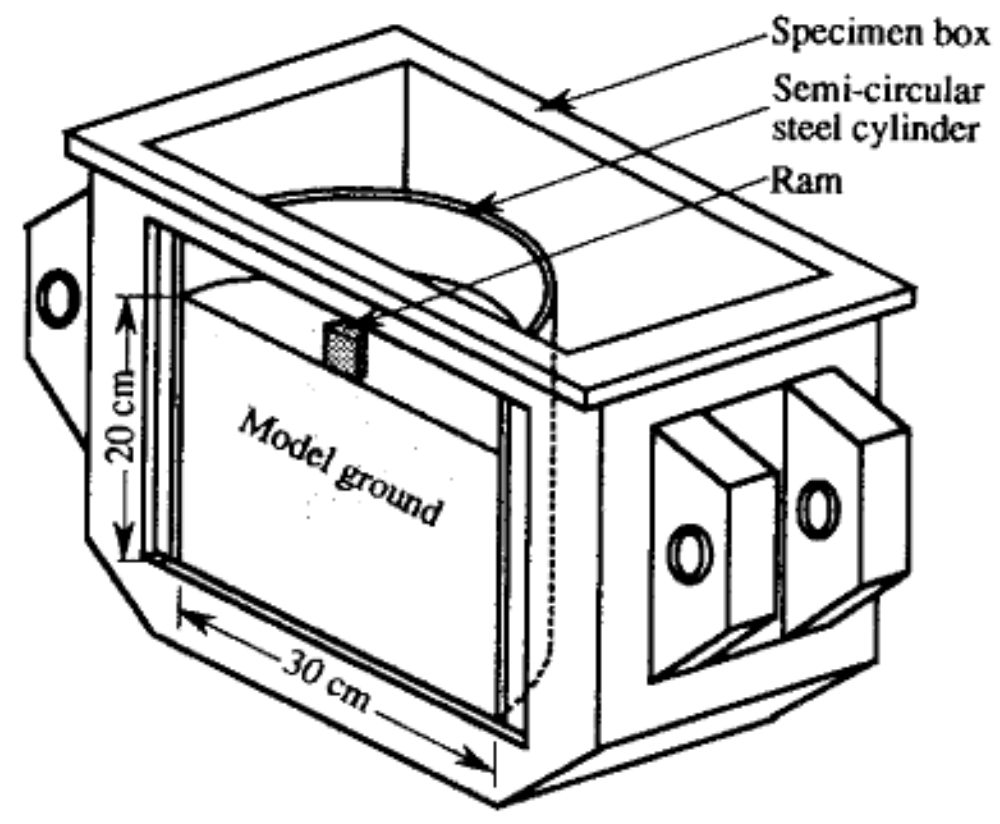

Figure 2-5 Model ground in centrifuge box (Oshima, 1994)

In addition, Oshima (1994) studied the ram acceleration during penetration through field measurements. A tamper with mass of 25 tons and base area of $4 \mathrm{~m}^{2}$ was dropped from heights $1,2,5$ and 10 meters. The tamper acceleration always exhibits a half sinusoidal shape. The contact time between the tamper and soil lasts for $0.050(\mathrm{sec})$ and is not sensitive to tamping energy level, as shown in Figure 2-8

$$
\begin{gathered}
P=c \frac{m v_{o}}{A} \\
P_{N}=c \frac{m v_{o}}{A} \sqrt{N}
\end{gathered}
$$




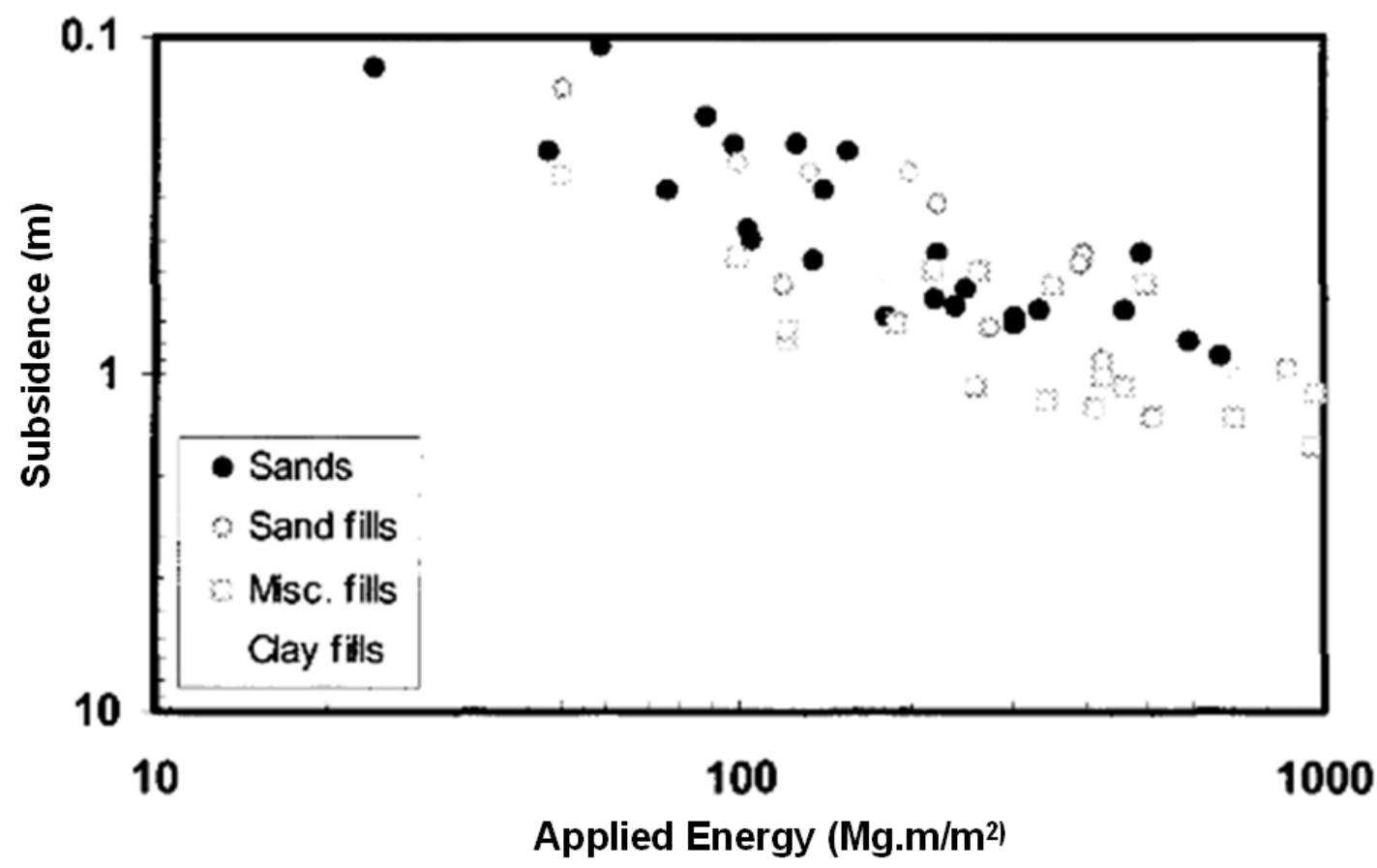

Figure 2-6 Effect of tamping energy on crater depth (Mayne et al. 1984)

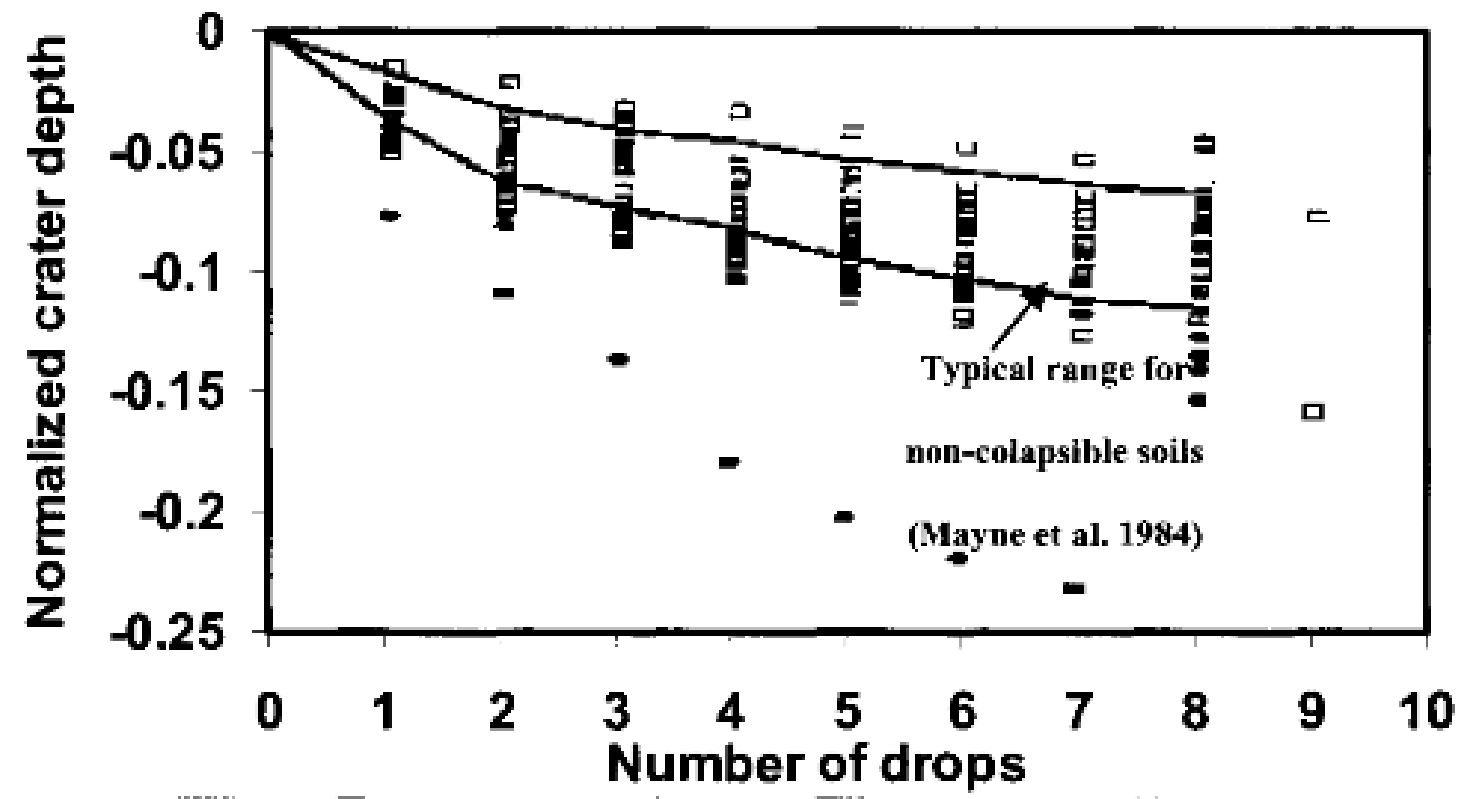

- Collapsibal soil after pre-w etting c Collapsipal soil at natural moisture

Figure 2-7 Normalized crater depth for collapsible soils (Rollins and Kim 1994) 


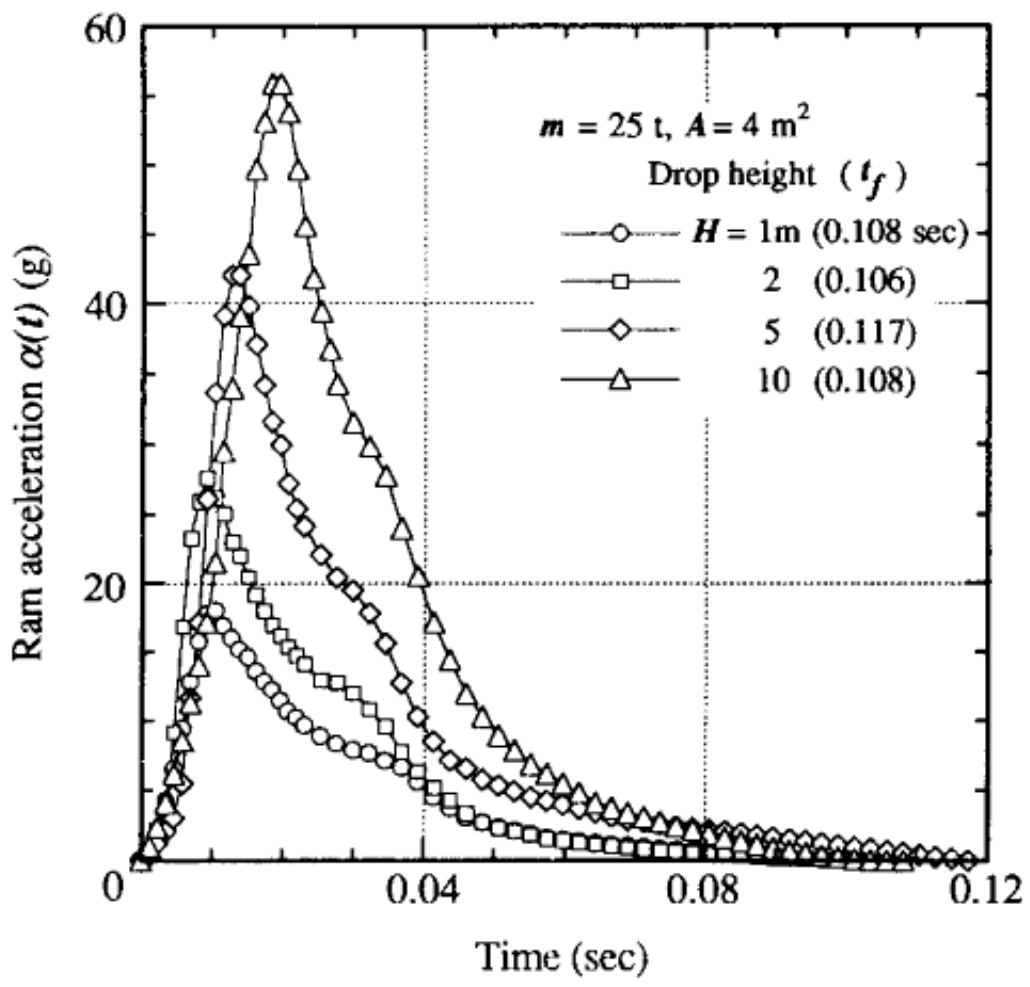

Figure 2-8 Measured tamper (ram) acceleration (Oshima, 1994)

\subsection{Induced Stresses}

Mayne et al. (1983) derived analytically a formula for estimating the induced stresses in the soil due to the impact of a falling weight. Using the conservation of momentum, the area under the force-time curve is equal to the change in momentum.

$$
\frac{1}{2} F_{\max } \Delta t=m \Delta V
$$

where

$F_{\text {max }}:$ Peak dynamic force

$\Delta t:$ Total time for deceleration

$m:$ tamper mass

$\Delta V:$ change in tamper velocity 
Since the tamper velocity at the end of tamping is zero, the change in tamper velocity is equal to the striking velocity and can be calculated using Newton's law $(V=\sqrt{2 g H})$. The contact time Mayne et al. work is equal to half of the natural period of the soil and can be calculated as follows:

$$
f_{n}=\frac{1}{T}=\frac{1}{2} \sqrt{\frac{k}{m}}
$$

where

$f_{n}:$ the natural frequency of soil

$T:$ is the period of vibration $(T=2 \Delta t)$

$k$ : Vertical stiffness of the system and equals to $k=4 G r_{o} /(1-v)$

$G$ : shear modulus

$r_{o}$ : tamper radius

$v$ : Poisson's ratio

By substituting $\Delta t=\frac{T}{2}$ into Equation (2-8) and then substitute $T$ from Equation (2-9), the maximum induced dynamic force can be calculated as follows

$$
F_{\max }=\sqrt{\frac{32 W H G r_{o}}{\pi^{2}(1-v)}}
$$

Mayne assumed that typical soil density and Poisson's ratio are 110 (pcf) and 0.37 respectively. A trapezoidal stress distribution due to tamping is also assumed. Then peak dynamic stress beneath the center of the tamper is approximately as:

$$
\sigma_{z \max }=\frac{V_{s} \sqrt{W H B}}{(B+z)^{2}}
$$


where

$V_{s}:$ Shear wave velocity $(\mathrm{m} / \mathrm{sec})$

$W:$ tamper of the falling mass (tones)

$B:$ tamper width $(\mathrm{m})$

$Z$ : Depth (m)

$H$ : Falling height (m)

The induced stress is proportional to the shear wave velocity and inversely proportional to the tamper width. Since shear wave velocity is different from one soil type to another, it may be concluded that Paul model was the first to relate the induced stresses to the initial soil properties.

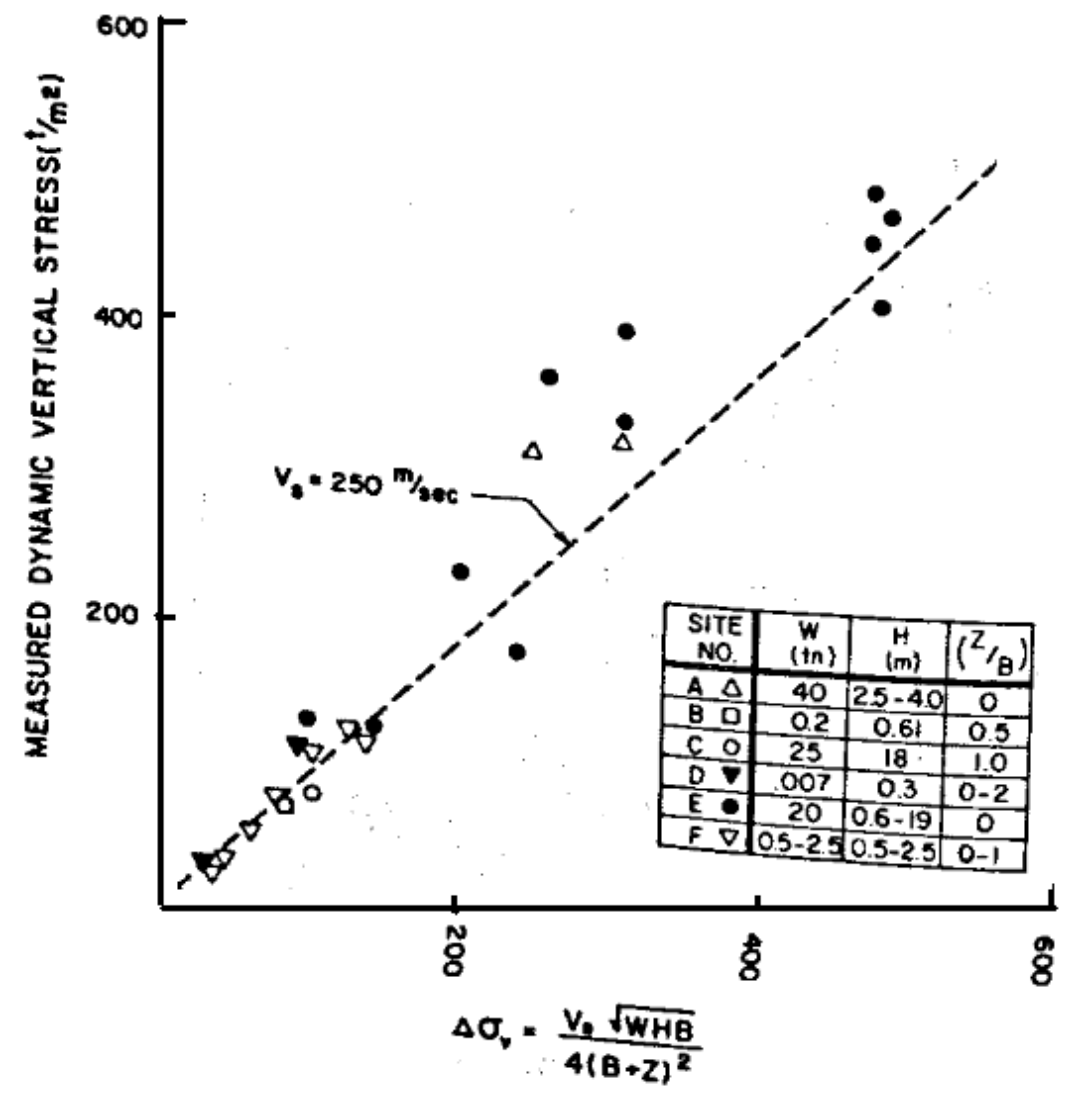

Figure 2-9 Comparison between predicated and measured peak transient dynamic stresses (Mayne,1983) 


\subsection{Induced Pore-pressure}

Gunaratne (1996) studied the change of pore-pressure during dynamic consolidation. An analytical model describing the pore pressure as a function of the depth and time was derived and given in Equation

(2-12). A computer program was written to execute Equation

(2-12). A parametric study was conducted to investigate the influence of the coefficient of consolidation $C_{V}$. Based on the parametric study, it was found that $C_{V}$ values for coarse soils do not allow significant pore pressure generation. On the other hand, $C_{V}$ values for clayey soil exhibit undrained behavior. Therefore, modeling of dissipation is essential for moderately pervious soils $\left(10-^{2}>C_{V}>10-^{4} \mathrm{in}^{2} . / \mathrm{s}\right)$

Laboratory experiments have been conducted to verify the analytical model. The model consisted of a tamper with $5 \mathrm{lb}$ and 3 in. square base. The tamper was held using electro-magnet. An electric switch is used to release the tamper and also to activate computerized measuring data acquisition. The stress is measured using stress cell attached to the base of the tamper. Pore pressure sensor was placed three inches beneath the soil surface. One cubic foot container was filled with organic soils which has relatively low hydraulic conductivity. The soil was compacted using vibratory process to ensure high saturation.

A good match has been achieved. The model was proven to be accurate for moderately low permeability soils. The variation of pore-pressure depends on the coefficient of consolidation $C_{V}$. It can be noticed from Figure 2-10 that the increase in pore pressure increases simultaneously with the induced transient stresses. The model 
was tested using sand properties. It was noticed that the induced pore pressure dissipates rapidly for soil type with high hydraulic conductivity. Gunaratne also reported that clayey impervious soils will exhibit undrained behavior.

$$
\begin{aligned}
& u(z, t)=\frac{2 B \Delta \sigma_{o}}{H} \sum_{n=1}^{n=\infty}\left[\int k(z) \sin \frac{N z}{H} d z\right] \sin \frac{N z}{H} \sum_{i=1}^{m 2} e^{-\frac{r C^{*}{ }_{v} N^{2}\left(t-t_{i}\right)}{H^{2}}} \\
& k(z)=\left[1-\frac{1}{\left[1+\left(\frac{r_{o}}{z}\right)^{2}\right]^{3 / 2}}\right]
\end{aligned}
$$

,and

$$
N=(2 n-1) \pi / 2
$$

where

$u(z, t)$ : Pore pressure at depth $z$ at time $t$

$B$ : Skempton's pore pressure parameter relevant to isotropic stress increments $r_{o}$ : tamper radius

$\Delta \sigma_{o}$ : Contact dynamic stress developed under a falling weight

$H$ : The height of the boundary

$N$ : Multiplier

$C_{v}^{*}$ : Coefficient of swelling (reverse consolidation) 


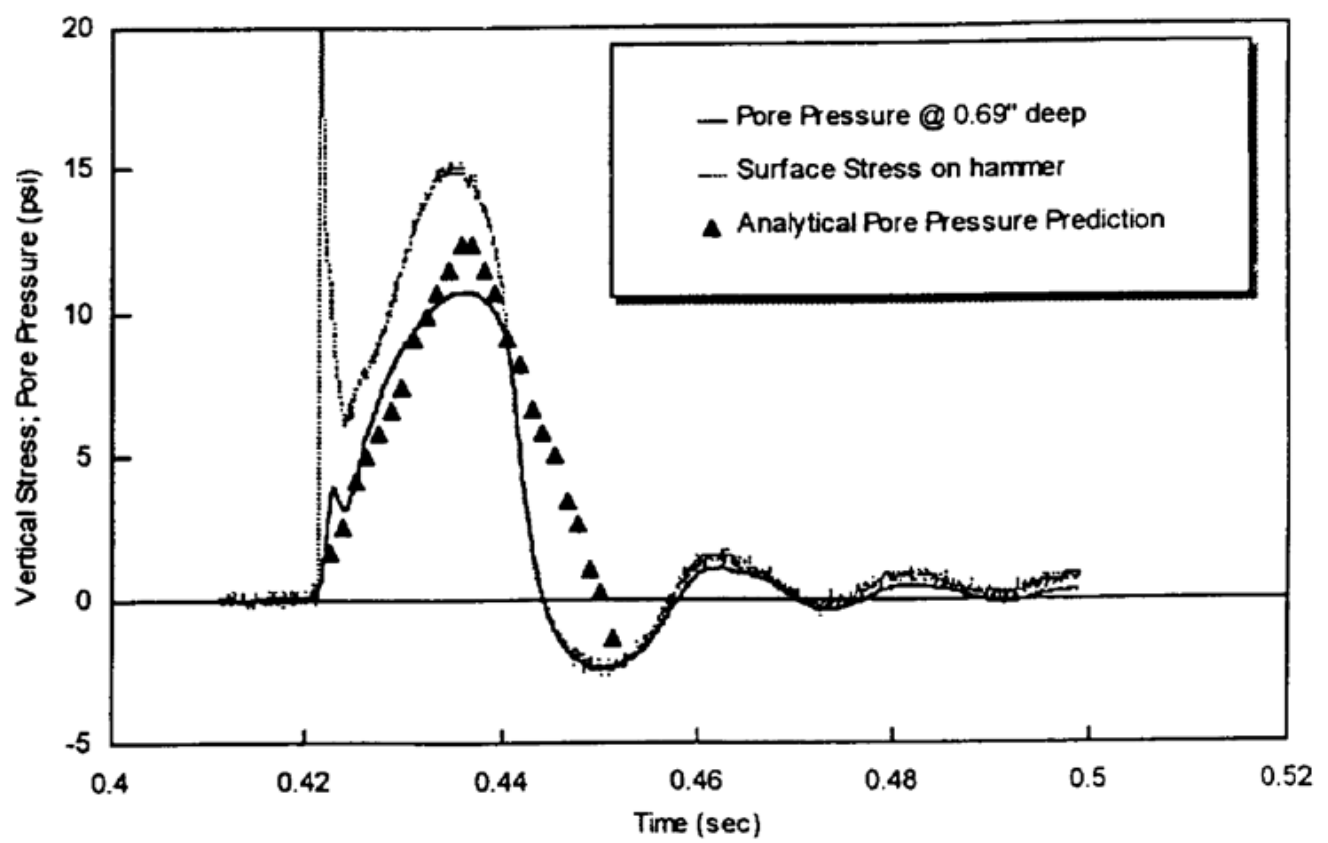

Figure 2-10 Comparison of predicted and measured pore pressure variation (Gunaratne, 1996)

Nashid (2005) presented an analytical simulation model for densification process of saturated sand deposits without wick drains and silty deposits supplemented by wick drains during DC. Pore pressure generated was simulated based on energy based liquefaction model. The consolidation theory was used to model the densification which occurs during densification. A computer code has been developed for the purpose of dynamic compaction simulation in silty soils. The tamping energy per blow, the number of tamping at each tamping spot, time cycle between drops and wick drain spacing effect on the densification process was investigated. Field experiments and case history was used to validate the model. Mitigation technique for the liquefaction that may be associated with the DC operations was proposed.

Querol et al. (2007) studied numerically the induced pore-pressure during DC operation in saturated sands. The work presented in the paper was based on the 
application of Pastor-Zienkiewicz (PZ) constitutive model for sands to dynamic consolidation problem. A finite element code was developed for the coupled fluid and soil phases displacement. Simulation results showed that water table oscillations were observed from their model. The wave of excess pore-pressure could be simulated successfully. Figure 2-11 shows the finite element model dimensions and boundary conditions.

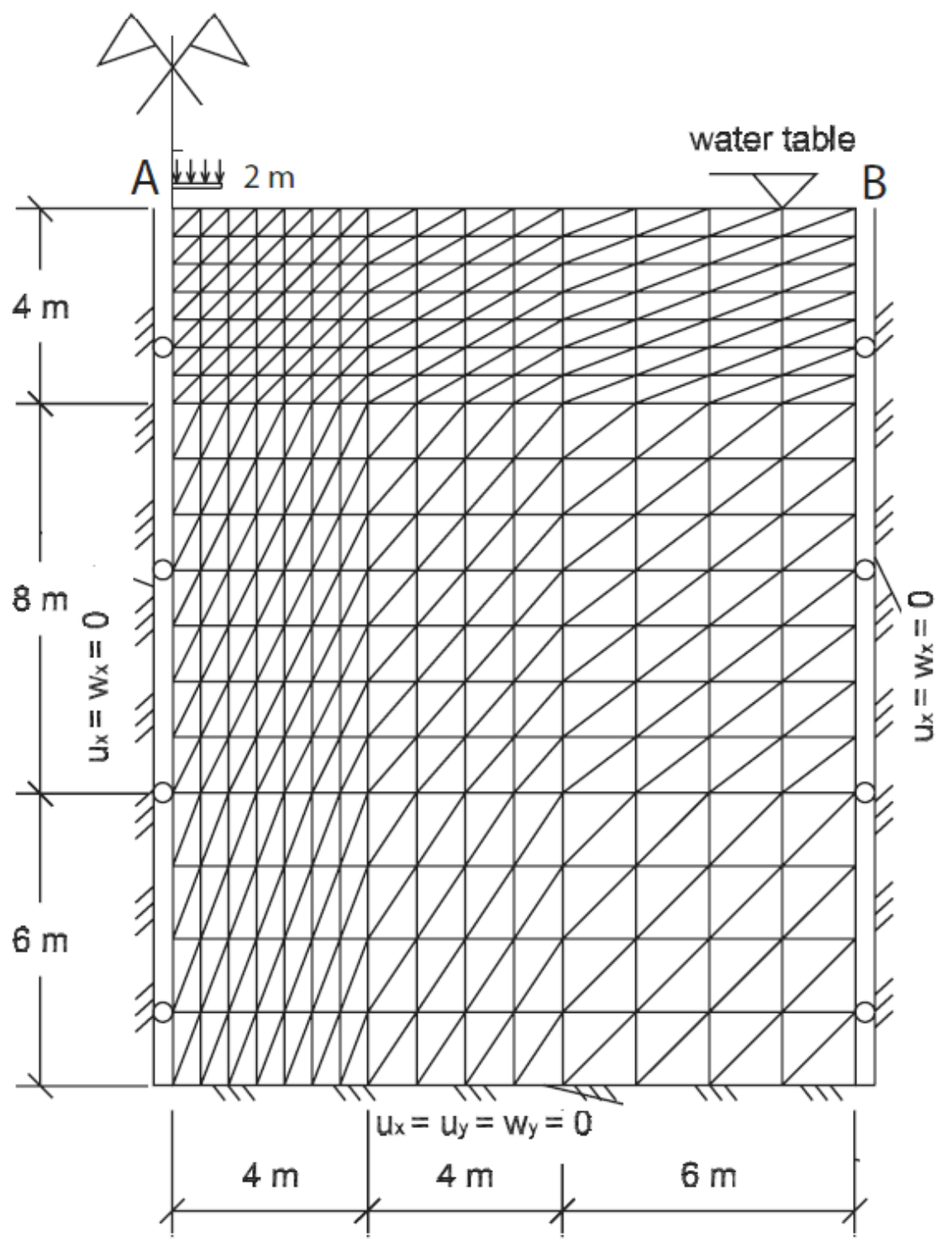

Figure 2-11 Querol et al.FE model 


\subsection{Ground Vibrations}

During the process of DC, waves are generated due to the impact of the falling mass onto the ground surface. Body and surface waves are generated and considered as an undesirable side effect to the adjacent buildings and structures. In addition to threaten the surrounding buildings, the generated waves carry the impact energy away from the treatment area resulting in reduced efficiency of DC. Therefore, the vibration amplitude and particle velocity of the traveling waves need to be estimated for the DC operation. Monitoring Peak Particle Velocity (PPV) is a necessity to avoid excessive vibration of buildings around the DC site. The limiting PPV for two story building is $50 \mathrm{~mm} / \mathrm{sec}$ which is considered one of the design limitations of DC process. This limitation was based on studying the vibration damage that was performed by Bureau of Mines and published in 1980.

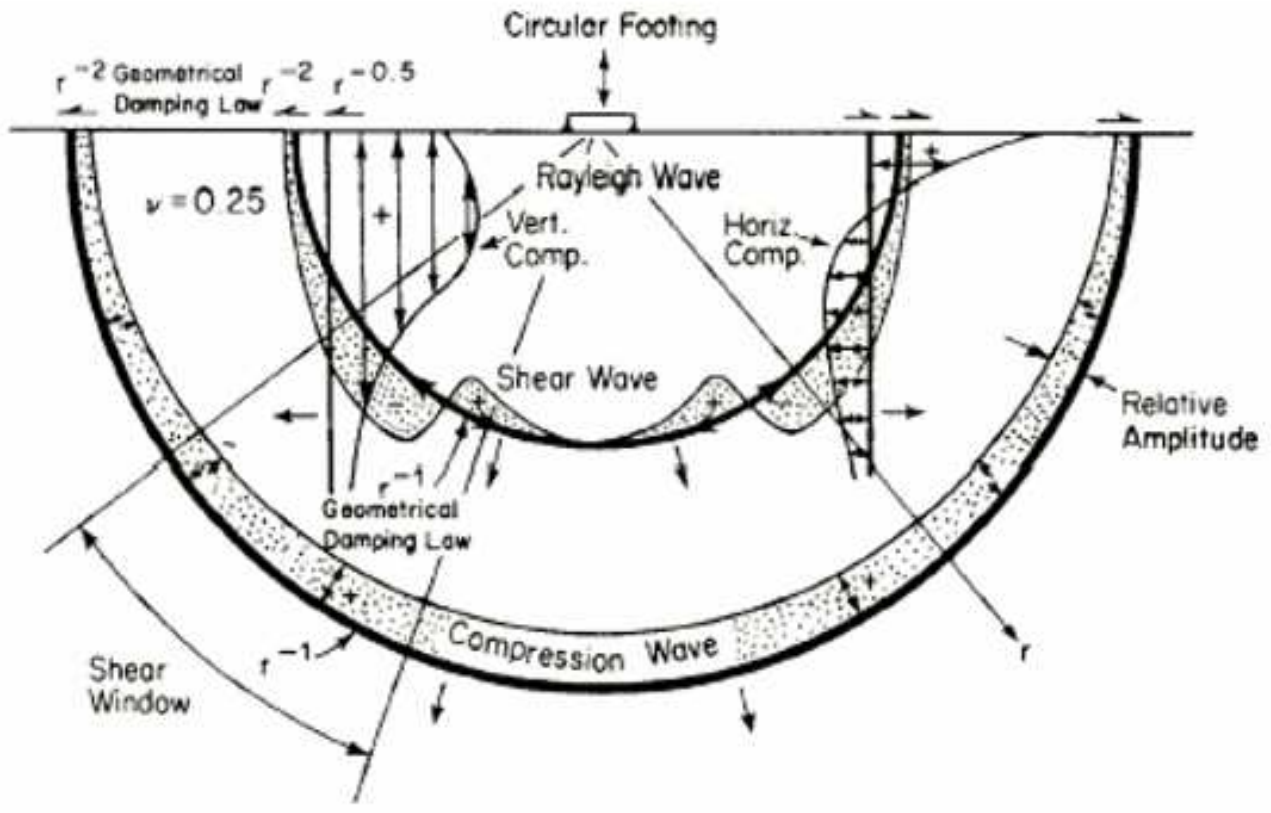

Figure 2-12 Waves generated during dynamic compaction, (Richart et al, 1970) 
The associated frequencies of the resulting wave range between $2-12 \mathrm{~Hz}$; the dominant frequency values are 3-4 Hz. Mayne (1984) reported that the wave velocity accompanying DC is usually slow close to the compaction area. The allowable peak particle velocities (PPV) are below $50 \mathrm{~mm} / \mathrm{sec}$ (Menrad, 1975). The primary source for this criterion came from blasting records form surface mining operation near residential communities. Note that the peak particle velocity is the maximum magnitude of velocity recorded at a measurement point. It can be calculated using the following formula

$$
P P V=\sqrt{x_{\text {max }}^{2}+y_{\text {max }}^{2}+z_{\text {max }}^{2}}
$$

where

$x_{\max }:$ Maximum particle velocity in $\mathrm{x}$ direction

$y_{\text {max }}$ : Maximum particle velocity in y direction

$z_{\max }$ : Maximum particle velocity in $\mathrm{z}$ direction

Mayne et al. (1984) utilized field measurements from 120 DC sites to derive a correlation between the impact energy and the PPV, which is reproduced in Figure 2-13 and Figure 2-14. The amplitude of PPV is inversely proportional to the distance measured from the center of impact, as shown in Equation (2-14). For soil types include silty sands, sandy fills, sandy clay, rubble, coal and debris fills, Mayne et al. reported another correlation given by Equation (2-15). Additional approach by the same authors is to normalize the PPV by the impact velocity as illustrated in Equation (2-16).

$$
P P V(\mathrm{~cm} / \mathrm{sec}) \leq 7\left(\frac{\sqrt{W H}}{d}\right)^{1.4}
$$




$$
\begin{gathered}
P P V(\mathrm{~mm} / \mathrm{sec})=92\left(\frac{\sqrt{W H}}{d}\right)^{1.7} \\
\frac{P P V}{\sqrt{2 g H}}=0.2\left(\frac{\sqrt{d}}{r_{o}}\right)^{-1.7}
\end{gathered}
$$

Rollins et al. (1994) proposed an expression for PPV given in Equation

(2-17). They also concluded that it is possible to conducted DC operation close to buildings when collapsible soils are treated. Lukas (1986) prepared a chart shown in Figure 2-15 using field measurements for different soil types. The normalized energy versus PPV is plotted with the level of disturbance indicated. The chart is considered a useful tool for DC field monitoring.

$$
P P V=20\left(\frac{\sqrt{W H}}{d}\right)^{1.03}
$$

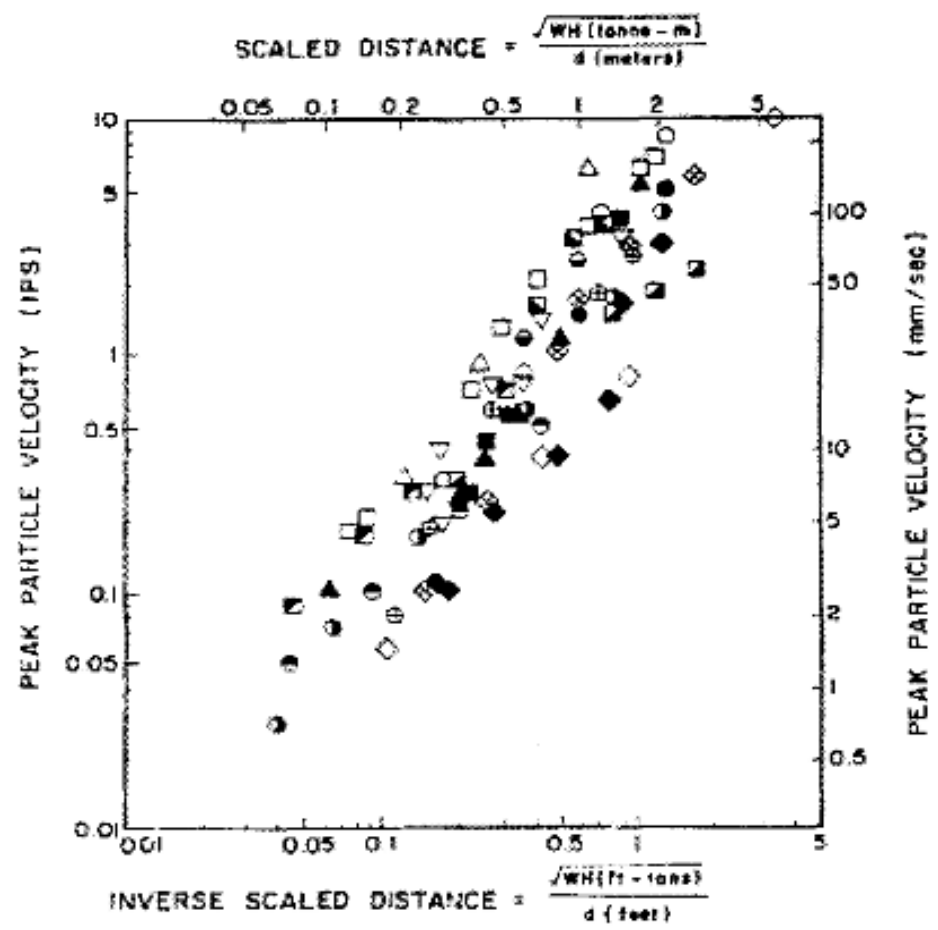

Figure 2-13 Attenuation of ground vibrations (Mayne 1985) 


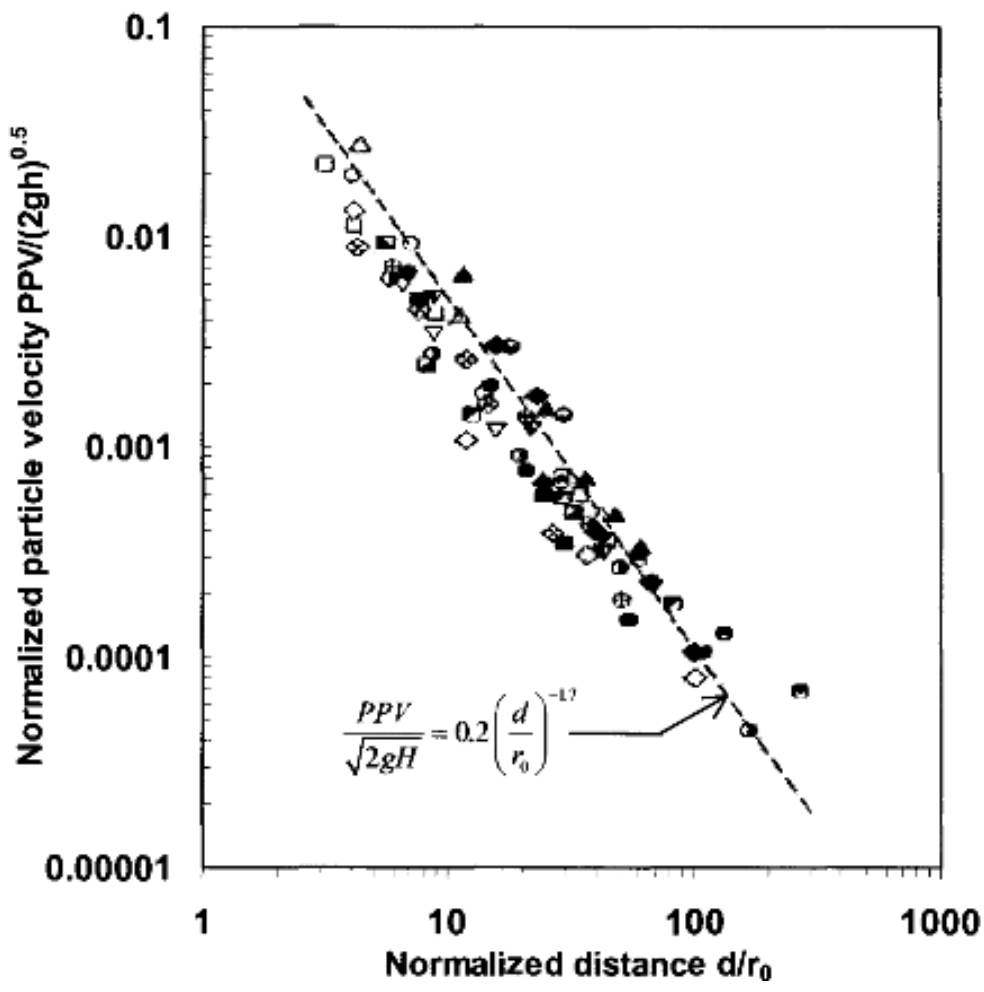

Figure 2-14 Attenuation of normalized PPV versus normalized distance (Mayne 1985)

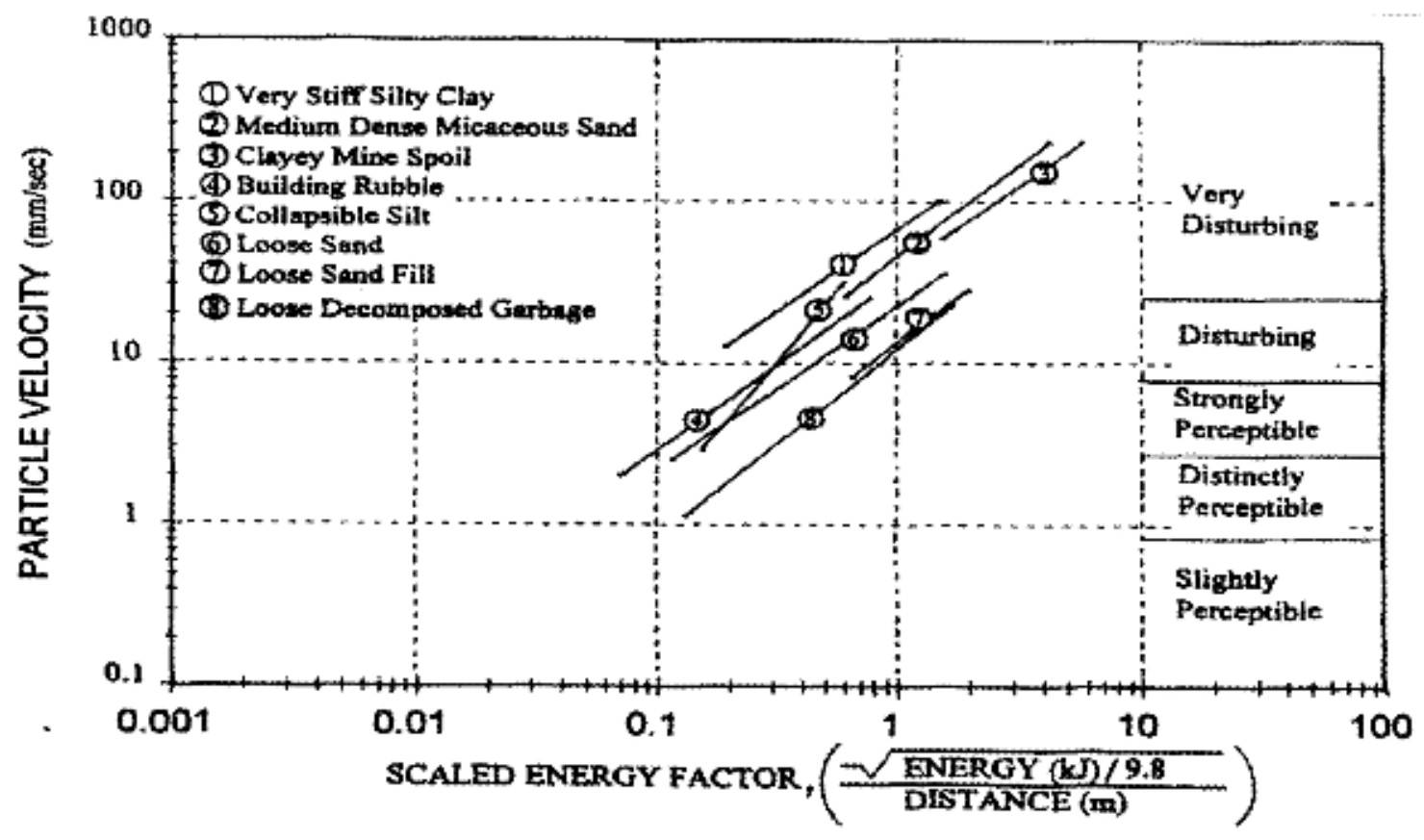

Figure 2-15 PPV for different soil deposits (Lukas 1986) 
Form the above discussion, it can be noticed that the current design models for estimating the PPV are simplified and independent of soil properties. Moreover, the same model may be used for different soil types. The work presented in this dissertation is concerned with understanding the influence of key soil properties, represented by the Cam-Clay constitutive model, on the PPV induced by DC.

\subsubsection{Attenuation}

Amplitude of all seismic waves attenuates as the distance form the impact increases. Part of the energy is attributed to the decrease in energy density caused by the distribution of wave energy on a continuously increasing area of the wave front. This is known as geometrical attenuation. During geometrical attenuation, the total energy of the wave front is constant with no loss. Body and surface waves are induced due to DC operation. Body waves (compression and shear waves) expand along hemi-spherical wave front. On the other hand, Surface waves (Raleigh and Love waves) expand in cylindrical wave front. Therefore, the energy density of the expanding body wave decreases at greater rate then the surface waves. Body waves attenuation is proportional to $1 / \mathrm{r}^{2}$ along the surface and $1 / \mathrm{r}$ elsewhere, while surface wave amplitude attenuation is proportional to $1 / \sqrt{r}$. More detailed discussion on wave attenuations can be found in Woods (1968) and Richard et al. (1970).

Analysis of energy dissipation in an ideal half space using a harmonic source for excitation shows that $67 \%$ of the wave energy transforms as Rayleigh wave. Considering the slower rate of geometrical attenuation, it becomes more reasonable and practically 
easier to concentrate on the propagation of surface wave. The dominance of Rayleigh wave on the propagation could also be noticed from Lamb (1904) work.

In addition to geometrical attenuation, ground vibration exhibits energy dissipation due to material damping. Material damping is defined by the attenuation coefficient $\alpha$. Woods et al. classified the soils into four classes ranging from sound hard rock to weak and soft soils based on the blow counts from SPT. For each class, attenuation coefficient was recommended. Since the attenuation coefficient is dependent on the frequency, two values were recommended for 5 and $50 \mathrm{~Hz}$, respectively.

To obtain the appropriate material attenuation coefficient for analysis, McDonal et al. (1958) approximated the material attenuation coefficient based on actual field test for variety of soil types. He reported that the attenuation coefficient is proportional to the frequency of the waves. For the same traveling distance, high frequency waves exhibits higher number of oscillation cycles resulting in higher dissipation rate of wave energy. Equation (2-18) shows McDonal et al. relationship to estimate the material attenuation factor $\alpha_{2}$ for a wave traveling at frequency $f_{2}$. The attenuation coefficient $\alpha_{1}$ is determined for different soil type using a wave of $f_{1}$ frequency and listed in McDonal et al. work.

Assuming the maximum observed frequency during DC is $10 \mathrm{~Hz} . \alpha 1$ in Equation (2-18) is attenuation coefficient for frequencies less that $f_{1}$ that was proposed by the McDonal et al. (1958). $\alpha 2$ is an approximation for the attenuation coefficient incase wave of a maximum $10(\mathrm{~Hz})$ frequency is traveling in the same medium. The coefficient $\alpha_{2}$ is used to define the material attenuation coefficient in ABAQUS FE code. 


$$
\begin{gathered}
\alpha_{2}=\alpha_{1}\left(\frac{f_{2}}{f_{1}}\right) \\
\alpha_{2}=0.01\left(\frac{10}{5}\right)=0.02 \mathrm{~m}^{-1}
\end{gathered}
$$

\subsection{Numerical Modeling of DC}

In this section, the modeling techniques for dynamic compaction in different types

of soils are reviewed, with particular emphasis on the modeling challenges and numerical difficulties due to the high strain level and large deformations in the Dc process. In general, it is noticed that using FE for modeling geotechnical engineering problem has received a lot of attention from the researchers because of its ability to capture highly non-linear behavior of soils. Additionally, the use of computer simulation saves the time and effort spent collecting field data. Poran et al. (1992) investigated the numerical modeling of successive, large deformation, rigid body impacts in dry sands. The modeling was performed by modeling a tamper as rigid elements. Two finite element codes were used to perform the study (DYNA2D and IMPACT) with a variety of soil models and computational algorithms. The large deformation and plastic behavior of sand during compaction required special computational techniques of re-meshing and the re-assignment of material properties, see Figure 2-16. In order to assess the ability of these two codes to model dynamic compaction, the numerical simulation results were validated using the available experimental data form extensive laboratory investigations. It was concluded that, FE results matched experimental results for loose sand. FE results deviated from the experimental results for dense sand or when the deformations are large associated with multiple impacts. Even with the proposed procedures for re-meshing and 
re-assigning material properties, the models were not able to capture the large deformations for dense sands. Finally, the authors advised that a special attention should be given to the selection of element size around the tamping area, because of its influence on the numerical accuracy and stability of the solution.

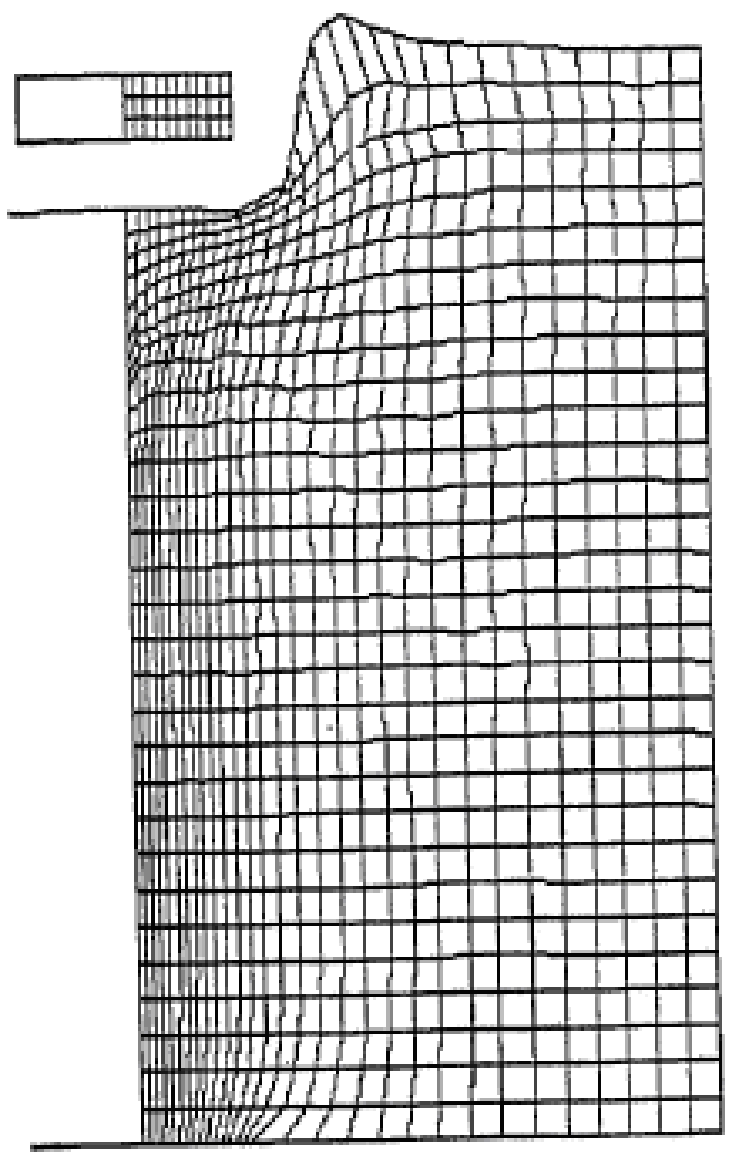

Figure 2-16 Deformed shape (Poran, 1992)

Pan (2002) simulated the dynamic compaction of loose soils under dynamic load numerically using ABAQUS. A full axi-symmetric elastic-plastic finite element model was generated. The dynamic compaction was modeled in two ways:(a) a force time input derived from the characteristic curve of the deceleration shape of the mass, and (b) a rigid body impacting onto the soil at the velocity varies with the drop height. Particle velocity 
at the ground surface, crater depth, the depth of influence were obtained and compared to those available from the literature. The force-time function is similar to damped sine wave or normal distribution function. The amplitude of the force-time function was calculated using Equation

(2-20) in which the kinetic energy of the tamper at striking is equal to the work done by the force-time function to induce the crater.

$$
\frac{1}{2} m v_{o}^{2}=\int F d z
$$

It was concluded that both modeling techniques can model P-wave propagation in the soil quite well. The model with rigid body impact over-predicts the depth of the effective treatment. The depth of influence was found to be $D I=2 \sqrt{W H}$ which is out of the common range $D I=0.5 \sqrt{W H}-1.0 \sqrt{W H}$. On the other hand, the model with input force predicted the crater depth more accurately but significantly overestimated the depth of influence $(D I)$. 


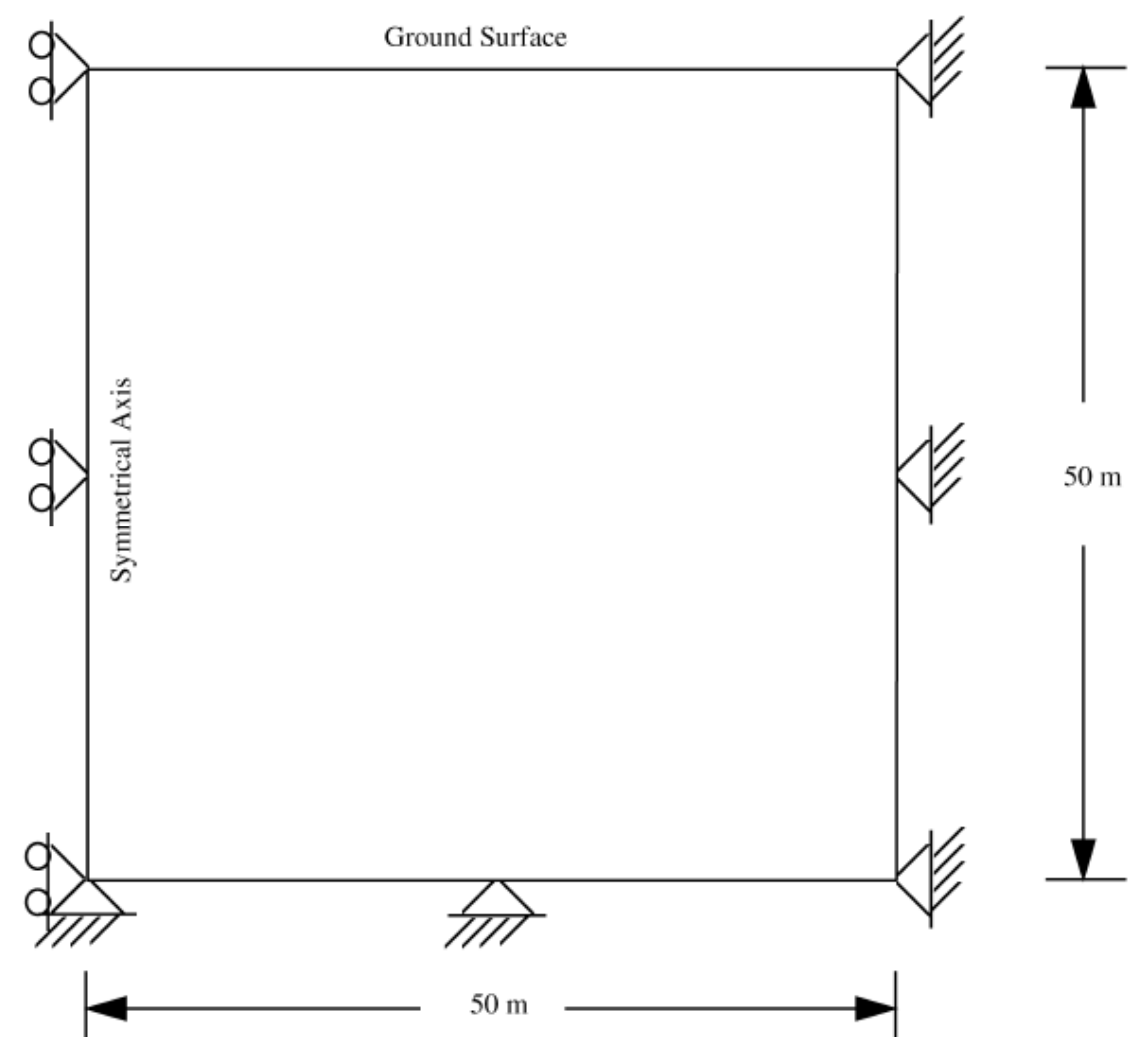

Figure 2-17 FE model dimensions and Boundary conditions (Pan, 2002)

$\mathrm{Gu}$ et al, (2002) studied the mechanics of dynamic compaction using twodimensional finite element analyses with large-strain dynamic formulation and a cap model for soil behavior. A realistic prediction of zone of improvement was achieved when it was compared to the results of centrifuge model. Tamping took place over a wooden plate using a tamper striking at initial velocity proportional to the drop height. Utilizing the work done by Oshima (1994), a half sinusoidal stress function was used to model multiple drops. It could be concluded that, with the appropriate selection of finite element formulation, algorithm and constitutive model, various aspects of dynamic compaction such as crater depth, depth and lateral extent of improvement can be captured. In an effort to understand the influence of tamper characteristics (mass, drop height and tamper area), a set of runs were conducted. Based on Gu et al. analysis, a 
tamper with larger mass dropped from lower drop height is recommended for increasing the depth of influence. Figure 2-18 shows Gu et al. model dimension, boundary conditions and mesh distribution.

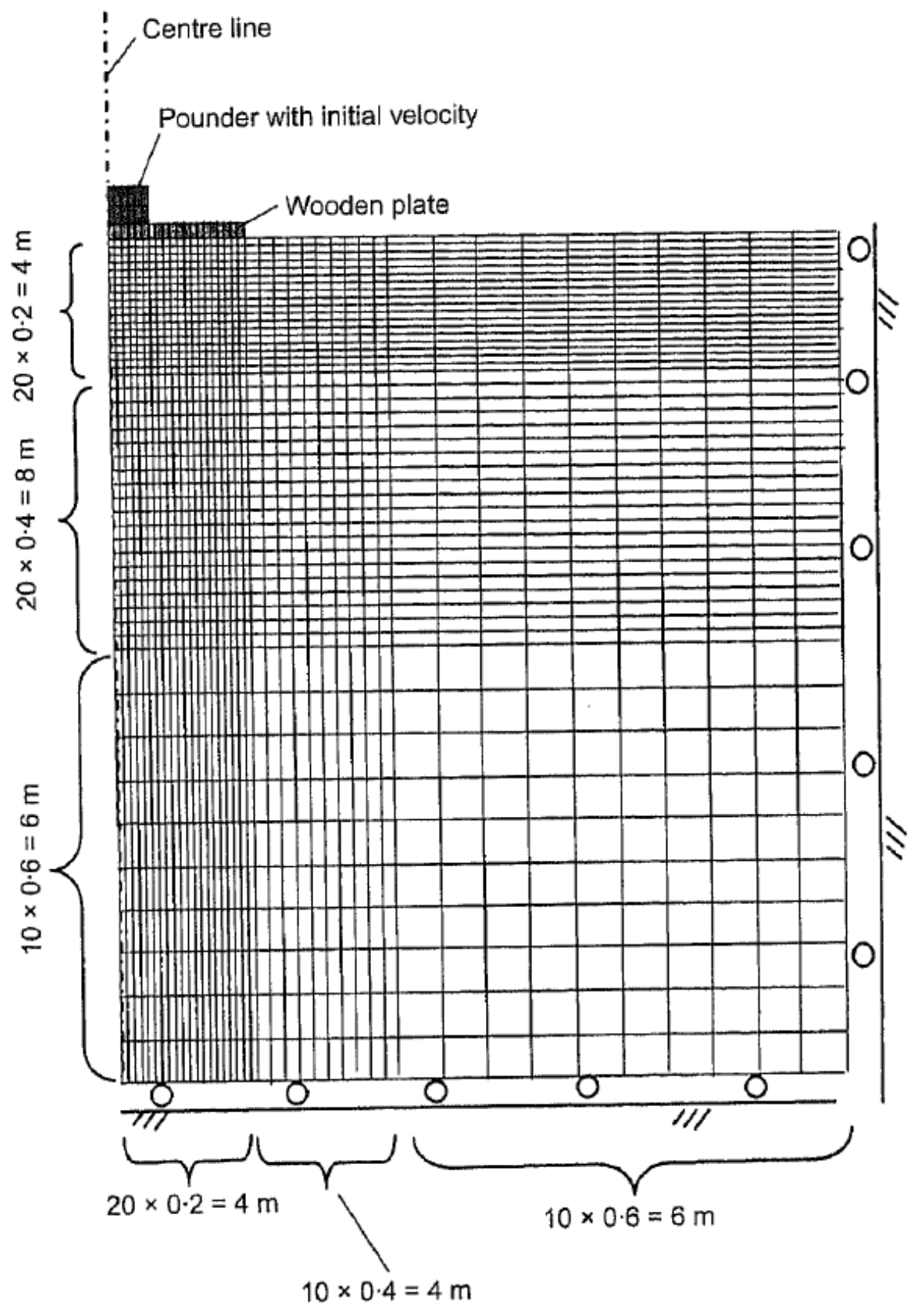

Figure 2-18 FE model dimensions and boundary conditions (Gu et al. 2002) 
Mesh size, or element size, should be carefully selected for the problem of wave propagation in a semi-infinite half space. Very small element size can cause numerical instability according to a study by Zerwer et al., (2003). On the other hand, large elements cannot allow the shorter waves to travel, which are associated with the highest frequencies. Vailliappan et al.(1984) indicated the minimum size to allow the propagation of short wavelengths. The time increment is selected, so that the primary wave, which is the fastest, can be recorded on two consequent nodes along the travel distance. Equations (2-21) and (2-22) provide mathematic expressions of the requirements.

$$
\begin{gathered}
g \leq \varsigma \lambda_{\min }=\varsigma \frac{V_{s}}{f_{\max }} \\
T \leq \frac{g}{V_{p}}
\end{gathered}
$$

where:

$g$ : Element size.

$V_{s}$ : Secondary wave velocity

$V_{p}$ : Primary wave velocity

$f_{\max }:$ Maximum input frequency

$\varsigma$ : Equals 0.25 for mass matrices are consistent and equals 0.2 for lumped masses

$T$ : Minimum sampling time

2.10. Soil Constitutive Models Used by Previous Finite Element Modeling

Soil constitutive models are the mathematical models that used to describe soil behavior under loading, unloading and reloading situations. The behavior of soils, in general, is known to be highly non-linear and rather complex since soil properties depend on the stress and strain levels. The current constitutive models were developed and validated based on field and laboratory experiments. 
Pan (2002) used the Mohr-Coulomb plasticity model available in the library of elements of ABAQUS FE code. The Mohr-Coulomb criterion assumes that failure occurs when the shear stress on any point in a material reaches a value that depends linearly on the normal stress in the same plane. The model is represented by a yield function of the Mohr-Coulomb form; this yield function includes isotropic cohesion ardening/softening. Cohesion, friction angle and dilation angle are used to define the model in ABAQUS FE code. Also the elastic behavior of the soil is defined by defining Young's modulus and Poisson's ratio. The model has been used extensively and for a wide range of geotechnical engineering problems. Pan identified the zone of influence by studying the stress and strain contours of the simulation results. Soil density after compaction was not studied. Pan et al. also concluded that, using Mohr-Coulomb lead to over estimation of the depth of influence.

Gu et al, (2002) studied DC numerically using CRISDYN (Goh, 1995). Cap plasticity model was used to simulate geological materials that exhibit pressuredependent yield and large-strain formulation for the dynamic problem was incorporated. Knowing that the loading is essentially impulsive and that the number of stress cycles (tamper drops) is likely to be small. Therefore, the cyclic densification features (the transition from contraction to dilatancy) were not incorporated in the constitutive model.

While the soil constitutive models are many, the Modified Cam-Clay constitutive model was used for the simulation conducted for the dissertation work. Cam-Clay constitutive model was developed by Roscoe and Schofield (1963) to model the elasticplastic behavior of cohesive soils. Then, it was modified by Roscoe and Burland (1968) to the modified Cam-Clay Model by modifying the shape of the yield surface. The main 
advantages of Cam-Clay model its ability to represent soil stress-strain behavior more realistically than other simplified models (Von-Misses or Mohr coulomb). In addition, softening and hardening behavior of cohesive soils can be captured. The model is described by a few parameters that can be obtained by using tri-axial laboratory test. 


\section{CHAPTER III}

\section{DEVELOPMENT OF MODELING TECHNIQUES WITH VALIDATIONS}

\subsection{Introduction}

A limited number of trials were presented in the literature to model the dynamic compaction process using FE code. Soil constitutive models used for these trials were limited to elastic-perfectly plastic models which are not able to capture the highly nonlinear behavior of soil. In addition, several modeling and re-meshing techniques were proposed to solve the problem of excessive deformation and elements distortion associated with modeling DC. In this chapter, modeling techniques for the problem of dynamic compaction in cohesive soils were presented. Cam-Clay constitutive model was used to capture the highly non-linear behavior of soils. The model also considers the preexisting state of vertical and horizontal stresses in the soil.

The objective of FE modeling is to investigate through intensive parametric study the influence of each Cam-Clay constitutive model parameters on the carter depth and the dimension of zone of influence. The influence of tamping energy per blow is studied as well. The influence of multiple drops is studied in the next chapter using an innovative modeling technique. This chapter is divided into three sections. The first section describes the development of FE model, including mesh convergence, and element size selection. The second section presents the modeling techniques for single and multiple 
drops situations. In the last section, FE models are validated using field measurements compiled from available literature.

\subsection{Description of 2-D FE Model}

Soil continuum is modeled as a semi-infinite half space. Due to the symmetry of the problem, one half of the geometry was modeled using $\sim 13,000$ 4-node bilinear axisymmetric elements. The model was constrained in the horizontal direction at the line of symmetry. Higher mesh density was used around impact zone to assure numerical accuracy. The boundary of the geometry domain is represented by infinite-elements to model far-field region. This type of elements absorbs the waves which arrive to the boundary and minimizes reflections that may occur if the boundary was fixed. The model is a quarter of a circle with $50 \mathrm{~m}$ radius, as shown in Figure 3-1. As was stated earlier in the literature review section, the practical limitation of the Peak Particle velocity is 50 $(\mathrm{mm} / \mathrm{sec})$ measured at distance 30 meters from the center of impact. In addition, pressure, shear and Rayleigh wave velocities are $(50,32$ and $29 \mathrm{~m} / \mathrm{sec})$ respectively. Model radius of $50 \mathrm{~m}$ is the minimum to allow the pressure wave to reach the boundaries and the Rayleigh wave, which causes the highest disturbance at the surface, to reach the $30 \mathrm{~m}$

point at the end of $1(\mathrm{sec})$ simulation time. Tamper, on the other hand, was modeled by using axi-symmetric rigid elements. Both horizontal and rotational degrees of freedom of the tamper were constrained. Modeling of tamping is described in Section 3-3. 


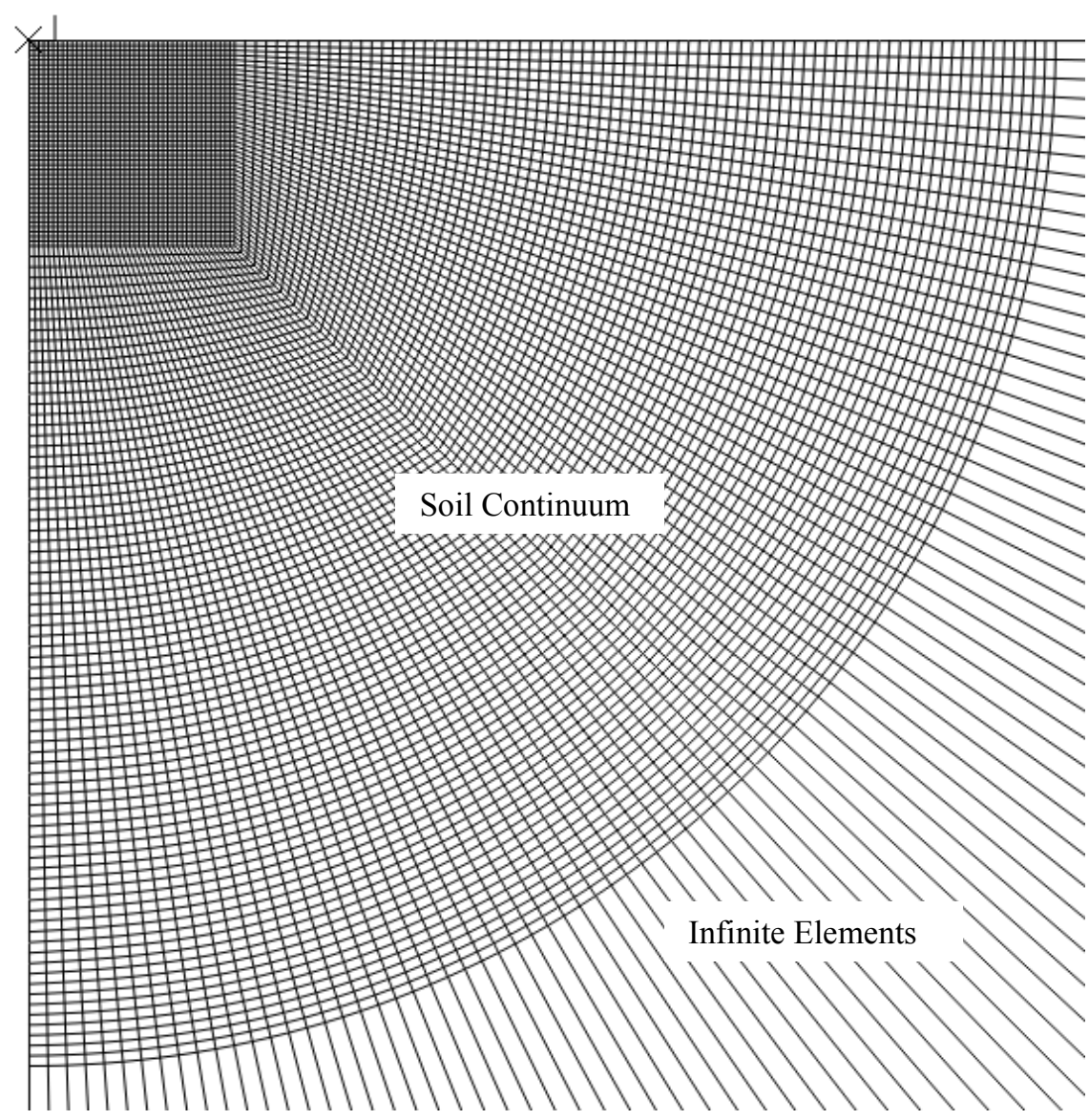

Figure 3-1 2-D axi-symmetric FE model

\subsubsection{Constitutive Model}

Modified Cam-Clay constitutive model was developed by Roscoe and Schofield (1968) for describing elastic-plastic behavior of cohesive soils. The main advantages of Cam-Clay model are based upon their ability to represent soil stress-strain behavior more realistically than other simplified models (Von -Misses or Mohr Coulomb). In addition, softening and hardening behavior can be captured. The behavior of clay can be divided into elastic behavior of the soil and plastic behavior. the yield surface which distinguish elastic and plastic has elliptical shape in the $p^{\prime}-\mathrm{q}$ plane, where $\mathrm{p}^{\prime}$ is the mean effective stress and $\mathrm{q}$ is the deviator stress. During loading, volume change occurs due to change in 
void volume. The relation between the change in void volume and the mean effective stress is governed by the logarithmic elastic bulk modulus $\kappa$ and logarithmic plastic bulk modulus $\lambda$. The most important advantage of Cam-Clay model is that at the end of each loading increment the radius of yield surface change which is known as the evolution law. The model is represented by a few parameters that can be obtained by using tri-axial laboratory test. Table 3-1 shows the baseline properties for the modified Cam-Clay which were obtained based on intensive survey of clay properties in the literature.

Table 3-1 Baseline properties of Cam-Clay parameters

\begin{tabular}{l|l|c}
\hline Parameter & Symbol & Base Line Value \\
\hline Soil density & $\rho(\mathrm{kg} / \mathrm{m} 3)$ & 2000 \\
\hline Poisson's ratio & $v$ & 0.25 \\
\hline Logarithmic plastic bulk modulus & $\lambda$ & 0.20 \\
\hline Logarithmic elastic bulk modulus & $\kappa$ & 0.02 \\
\hline Initial yield surface & $a_{0}(\mathrm{kPa})$ & 50 \\
\hline Critical state line slope & $M$ & 1.00 \\
\hline Initial void ratio & $e_{0}$ & 1.0 \\
\hline
\end{tabular}

\subsubsection{Geostatic Step and Dynamic Integration Scheme}

Geostatic analysis is a built in step (analysis) in ABAQUS FE code. It is normally used as the first step in geotechnical engineering problems. By applying gravity load, the state of equilibrium between the applied gravity load and soil stresses should be reached at zero deformations. The initial conditions are defined by calculating the vertical and horizontal stresses using specific soil weight and coefficient of lateral earth pressure as shown in Equations (3-1) and (3-2). Lateral earth pressure coefficient is a function of soil friction angle which is related to the critical state line slope (M) using Equations (3-3) and (3-4). Initial void ratio distribution is also defined during geostatic step analysis and assumed constant from the ground surface down. Figure 3-2 shows the vertical and 
horizontal stresses as a function of depth as calculated using ABAQUS Geostatic analysis step.

Subsequent to applying geostatic stresses, tamping is modeled in a second step (analysis) using dynamic analysis option. ABAQUS offers several methods for performing dynamic analysis of problems in which inertia and damping effects are important. Dynamic/Implicit was used throughout the work presented in this report. Dynamic/standard uses implicit time integration to solve nonlinear problems. Therefore, it is recommended for highly nonlinear problems and when high solution accuracy is desired. Moreover, because of the large deformations, non-linear geometry option was used for the analysis which updated element stiffness matrix after each time step of the solution. Vertical stress in the soil continuum can be calculated as shown in Equation (3-1). The vertical stress varies linearly with depth. The horizontal stress is proportional to the vertical stress. The lateral earth pressure $K_{o}$ indicates the ratio between horizontal to vertical stress, as shown in Equation (3-2). The lateral earth pressure is also a material dependant parameter and is related to soil friction angle $\phi$, Equation (3-3). The friction angle can be related to slope of critical state line in the Cam-Clay constitutive model using the Equation (3-4). Equations (3-1) to (3-4) were used to define the initial state of stress that exists in the soil.

$$
\begin{gathered}
\sigma_{v}=\gamma H \\
\sigma_{h}=K_{o} \sigma_{v} \\
K_{o}=(1-\sin \phi)
\end{gathered}
$$




$$
\sin \phi=\frac{3 M}{6-M}
$$

where

$\sigma_{v}:$ Vertical Stress

$\gamma:$ soil specific weight

$H$ : depth measured from the ground surface

$K_{o}$ : lateral earth pressure coefficient at rest

$\phi$ : friction angle

$M$ : slope of critical state line of Cam-Clay constitutive model

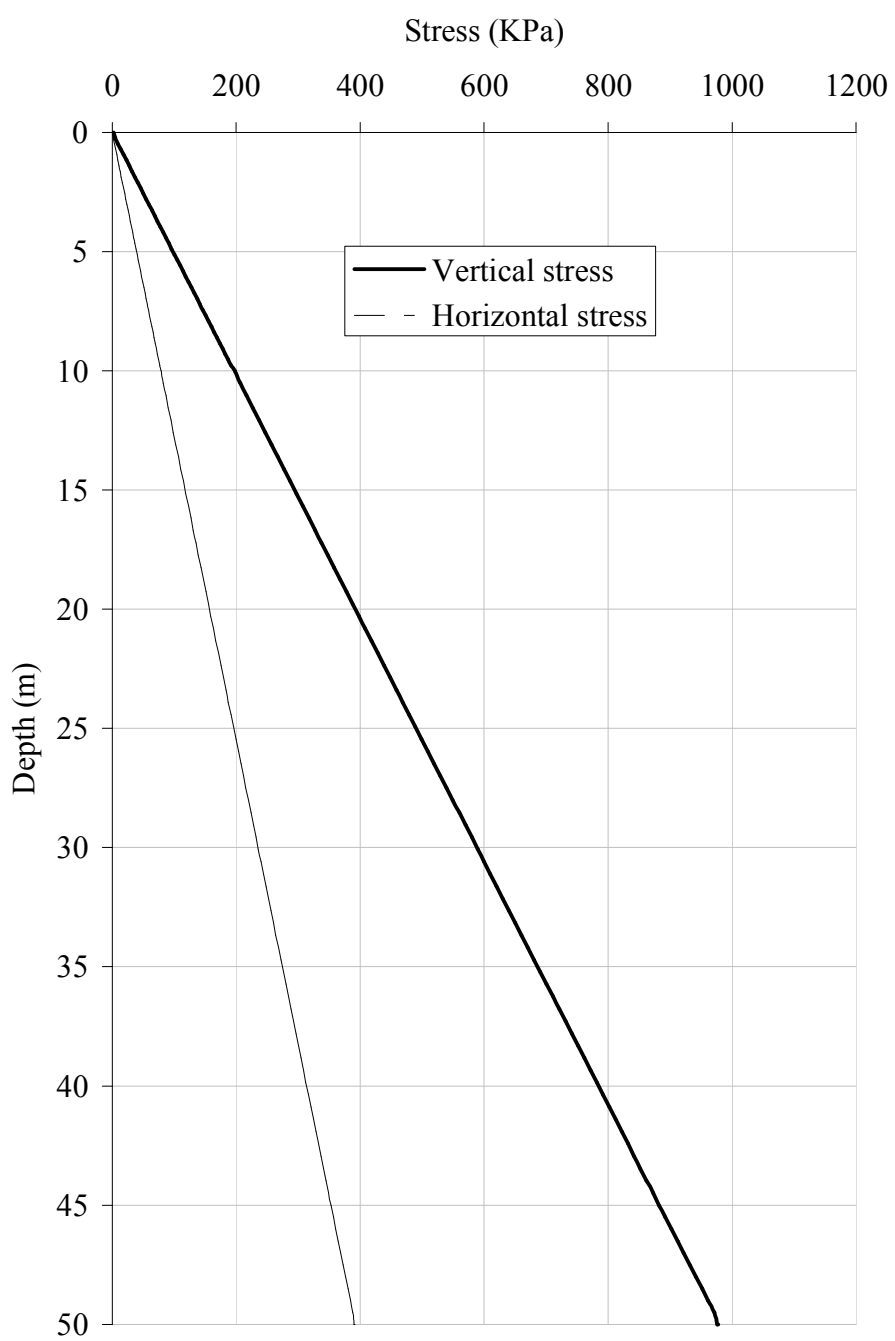

Figure 3-2 Initial state of stress (Geostatic step) 


\subsubsection{Mesh Convergence and Model Dimensions}

Finite element is a numerical method in which the geometry of the problem is discretized into small unit called "elements' which are connected to each other at shared points called "nodes". The solution accuracy and the success of modeling dynamic problems depend on element size. Coarse elements are stiffer than fine elements. Also, element shape influences the accuracy of solution for the same reason. Elongated or sharp angled elements tend to be stiffer. In addition, especially if large deformation occurs, element gets highly elongated or sharp angled, which tend to lead to simulation crash. Therefore, special attention needs to apply to the selection of the element size and shape.

Mesh size, or element size, should be carefully selected for the problem of wave propagation in a dynamic compaction model. Very small element size can cause numerical instability, based on the study by Zerwer et al. (2003). On the other hand, large elements cannot allow the shorter wave to travel, which are associated with the highest frequencies. Vailliappan et al. (1984) suggested the minimum size to allow the propagation of short wavelengths. In addition, the time increment should be selected so that the fastest wave (pressure wave) can be recorded on two consecutive nodes along the travel distance. (See Equations (2-18) and (2-19)).

Primary wave propagation velocity,

$V_{p}=\sqrt{\frac{E}{\rho}}=\sqrt{\frac{5 \times 10^{6}}{2000}} \approx 50(\mathrm{~m} / \mathrm{sec})$

Shear wave propagation velocity,

$V_{s}=\sqrt{\frac{G}{\rho}}=\sqrt{\frac{E}{2 \rho(1+v)}}=\sqrt{\frac{5 \times 10^{6}}{2(1+.25) 2000}} \approx 31.6(\mathrm{~m} / \mathrm{sec})$ 
Then, Rayleigh wave propagation velocity,

$V_{R}=0.9192 V_{s}=29(\mathrm{~m} / \mathrm{sec})$

Assuming that the maximum frequency of waves is $10 \mathrm{~Hz}$, using Vailliappan (1984) formula, the minimum element size equals:

$g \leq \varsigma \lambda_{\min }=\varsigma \frac{V_{R}}{f_{\max }}=0.25 x \frac{29}{10}=0.725(\mathrm{~m})$

Since $\varsigma=0.25$ for continuum medium, the sampling time for simulation equals:

$T \leq \frac{g}{V_{p}}=\frac{0.725}{50}=0.0145(\mathrm{sec})$

According to Zewer (2003) formula, elements size of 0.724 (m) is enough to model the wave propagation which is initiated during tamping. However, additional convergence check is needed. Therefore, four runs were used to model the DC using tamper mass $=10$ tons dropped from 10 meters height to study the mesh convergence. Two criteria were used to select the element size: void ratio distribution beneath the tamper and crater depth. Figure 3-3 shows the variation of the crater depth with the element size for the baseline model properties. Figure 3-4 shows the resulting void ratio distribution (the vertical axis represented the depth measured from the crater bottom). It can be noticed that an element size of 0.5 meters would be sufficient since there was no change in crater depth and void ratio distribution. Therefore, element size $0.4(\mathrm{~m})$, which corresponds to $\sim 13,000$ elements for the entire problem domain, was used for the $\mathrm{FE}$ model. The time increment time was selected to equal to 0.001 (sec). 


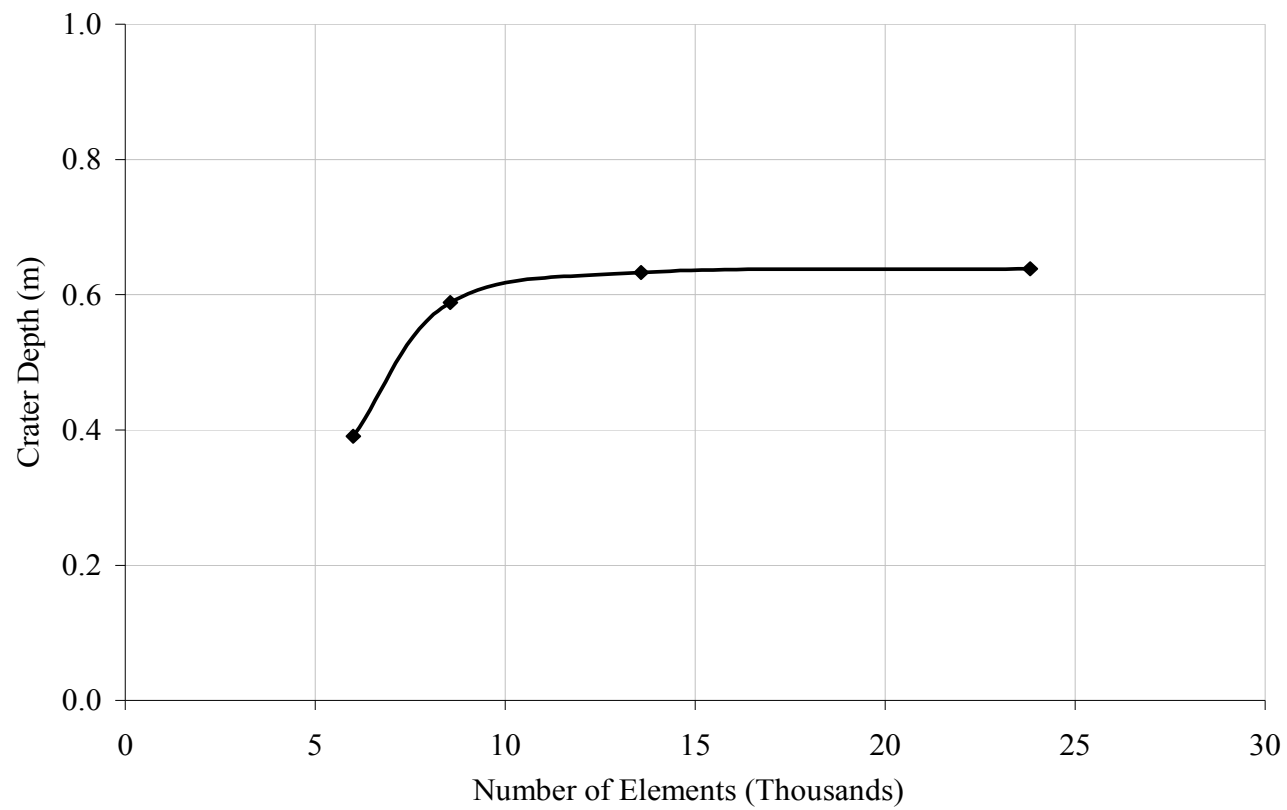

Figure 3-3 Mesh convergence (crater depth) 


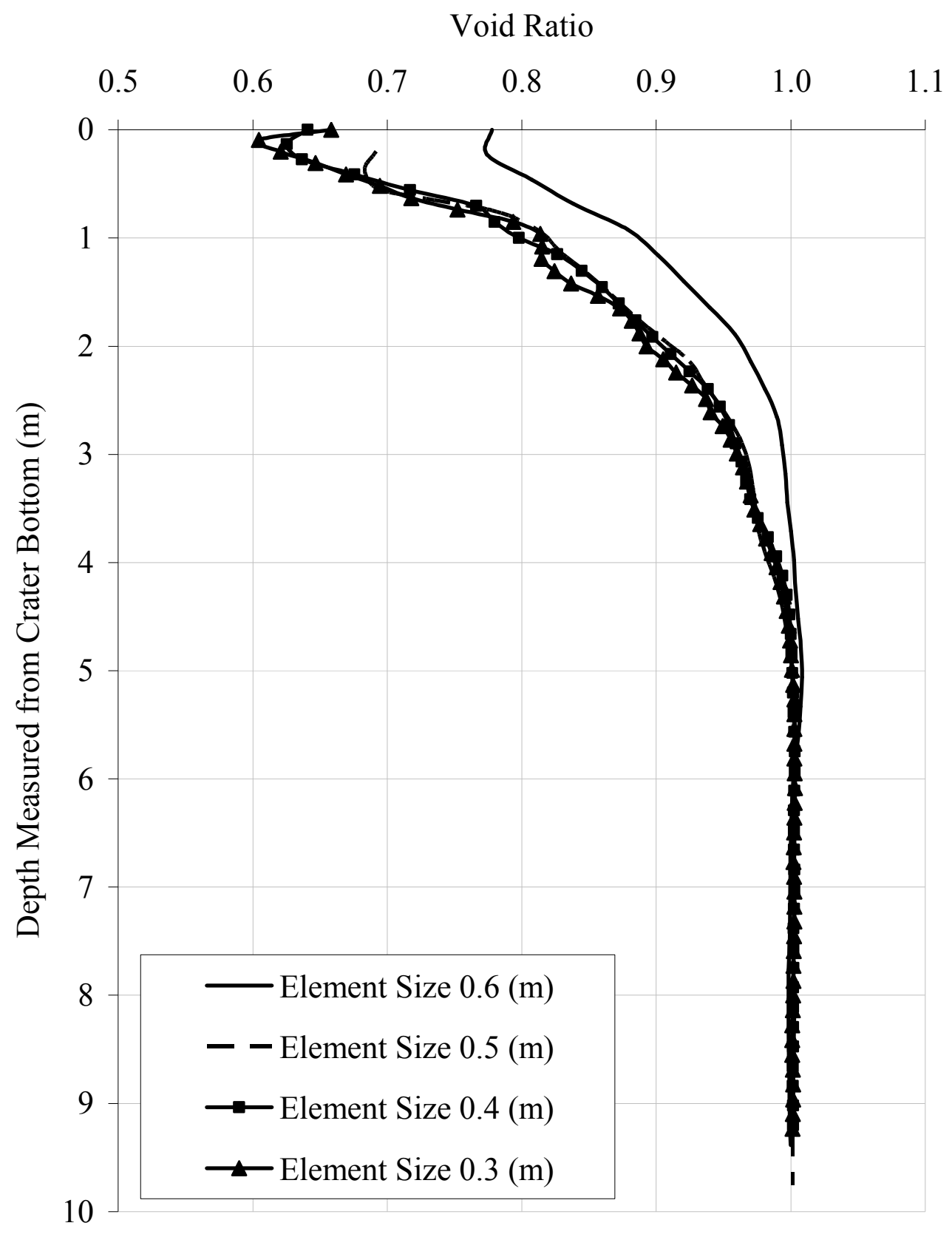

Figure 3-4 Mesh convergence (void ratio distribution beneath the tamper) 


\subsection{Modeling of Tamping}

It was stated earlier that tamper was modeled using axi-symmetric rigid elements. The selection of rigid element is based on the fact that tampers are usually made of steel or concrete blocks which have much higher stiffness than soils. Rigid elements are weightless members that are used to transfer forces and, at the same time, are not allowed to deform. The interaction between soil and tamper was modeled using general interaction scheme in ABAQUS interaction module in which tangential and normal components were defined. A friction coefficient of 0.05 is assumed in the horizontal direction. Few runs were used to estimate the influence of friction coefficient value. The influence was found to be minimal. The interaction component in the normal direction was assumed to be hard with no separation option activated. In this section, two modeling techniques for compaction (tamping) are presented.

\subsubsection{Tamping with Initial Velocity}

In this model, mass of the tamper was modeled as a rigid element with concentrated mass at the center of symmetry. The tamper impacts soil continuum surface by giving it initial velocity $V_{o}$. where:

$V_{o}:$ tamper velocity at the striking

$H$ : Drop height

$g$; Ground acceleration $(=9.81 \mathrm{~m} / \mathrm{sec} 2)$

Table 3-2 shows the velocity of contact as a function of drop height which is used for modeling single drop cases. The striking velocity can be calculated for the free falling tamper using Newton's Law as $V_{o}=\sqrt{2 g H}$ 
where:

$V_{o}:$ tamper velocity at the striking

$H$ : Drop height

$g$; Ground acceleration $\left(=9.81 \mathrm{~m} / \mathrm{sec}^{2}\right)$

Table 3-2 Impact velocities $(\mathrm{m} / \mathrm{sec})$

\begin{tabular}{l|c|c|c}
\hline & Mass (tons) $=10$ & Mass (tons) $=15$ & Mass (tons) $=20$ \\
\hline Height $(\mathrm{m})=10$ & 14.00 & 14.00 & 14.00 \\
\hline Height $(\mathrm{m})=15$ & 17.15 & 17.15 & 17.15 \\
\hline Height $(\mathrm{m})=20$ & 19.80 & 19.80 & 19.80 \\
\hline
\end{tabular}

\subsubsection{Tamping with Force-time Function}

ABAQUS FE code, unfortunately, can not model the case of multiple tamping. Therefore, an alternative approach was proposed by Pan (2002) for handling this problem. Pan utilized the work done by other researchers who concluded that half sinusoidal shaped stress function is induced beneath the tamper. The force-time function is then repeatedly applied to model the multi drop (tamping) case. The magnitude of the force-time function is equal to stress induced underneath the tamper calculated from the initial velocity model. Section 2-7 contains more details about Pan's model and assumptions.

The force-time function is used herein to model for the multiple tamping case. The magnitude of the force-time function is obtained by performing some iteration using the developed FE model. For each iteration, a different magnitude was used then the results were compared with the results of the model in which soil is tamping using rigid mass assigned initial velocity. The initial-velocity model that was used for the comparison has a tamper mass equal 10 tons dropped from 10 meters height. The equivalent pressure magnitude was found to be equal to $1.25 \times 10^{6}(\mathrm{~Pa})$ applied to the 
tamper area which is equal to $4.9 \mathrm{~m}^{2}$. Figure 3-5 shows the Pressure-time function used in ABAQUS FE code for the analysis. Figure 3-6 and Figure 3-7 compare solution from the force-time function and initial velocity models. Figure 3-6 shows the induced crater depth by two models. It can be noticed that simulation of tamping using force-time function could achieve similar results as tamping using the initial velocity. Also, as can be noticed from Figure 3-7, void ratio distribution beneath the tamper for the both cases is close. Therefore, a force-time function with amplitude equal to $1.25 \times 10^{6}(\mathrm{~Pa})$ was considered to be equivalent to tamping with 10 tons tamper dropped from 10 meters height.

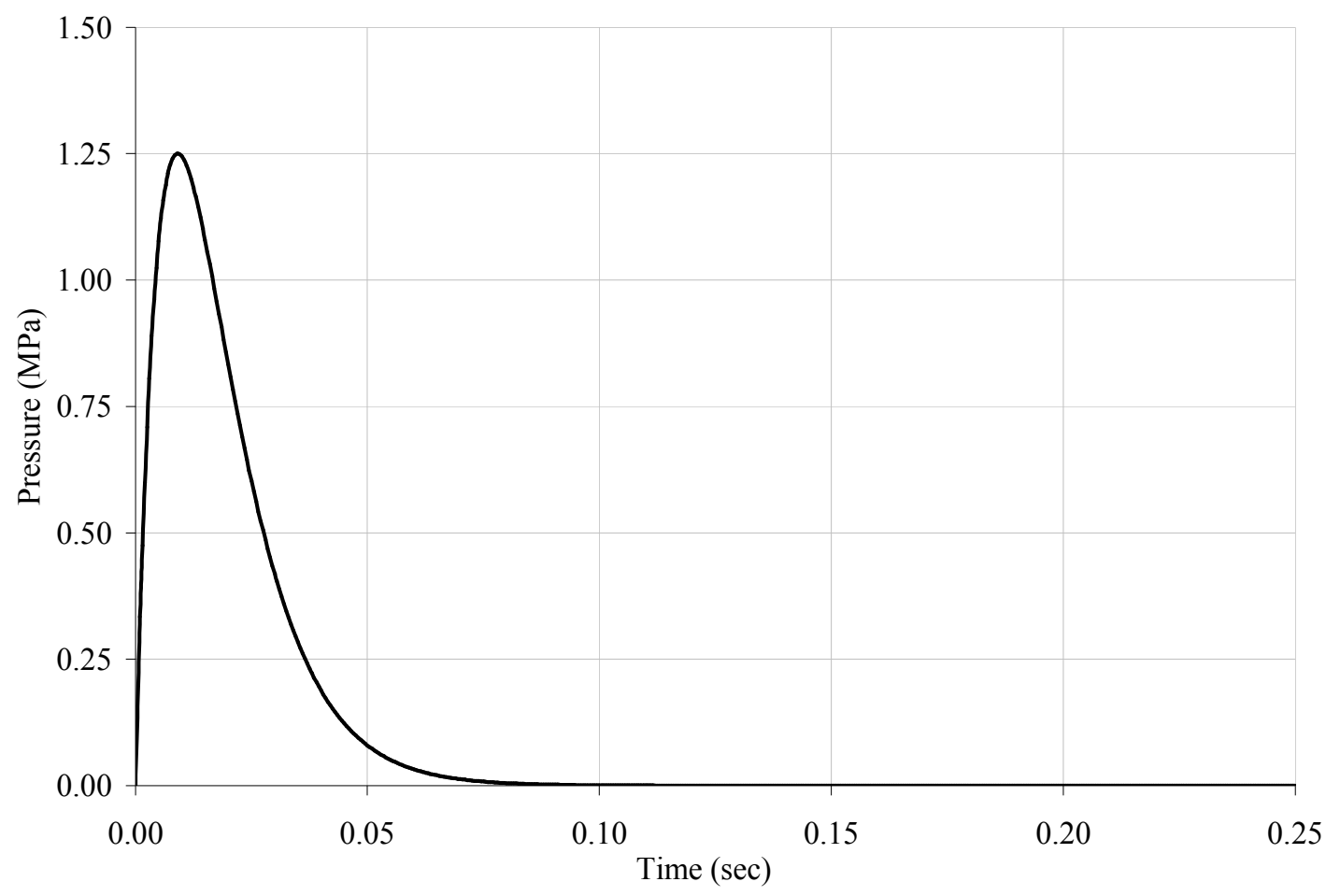

Figure 3-5 Transient pressure load 


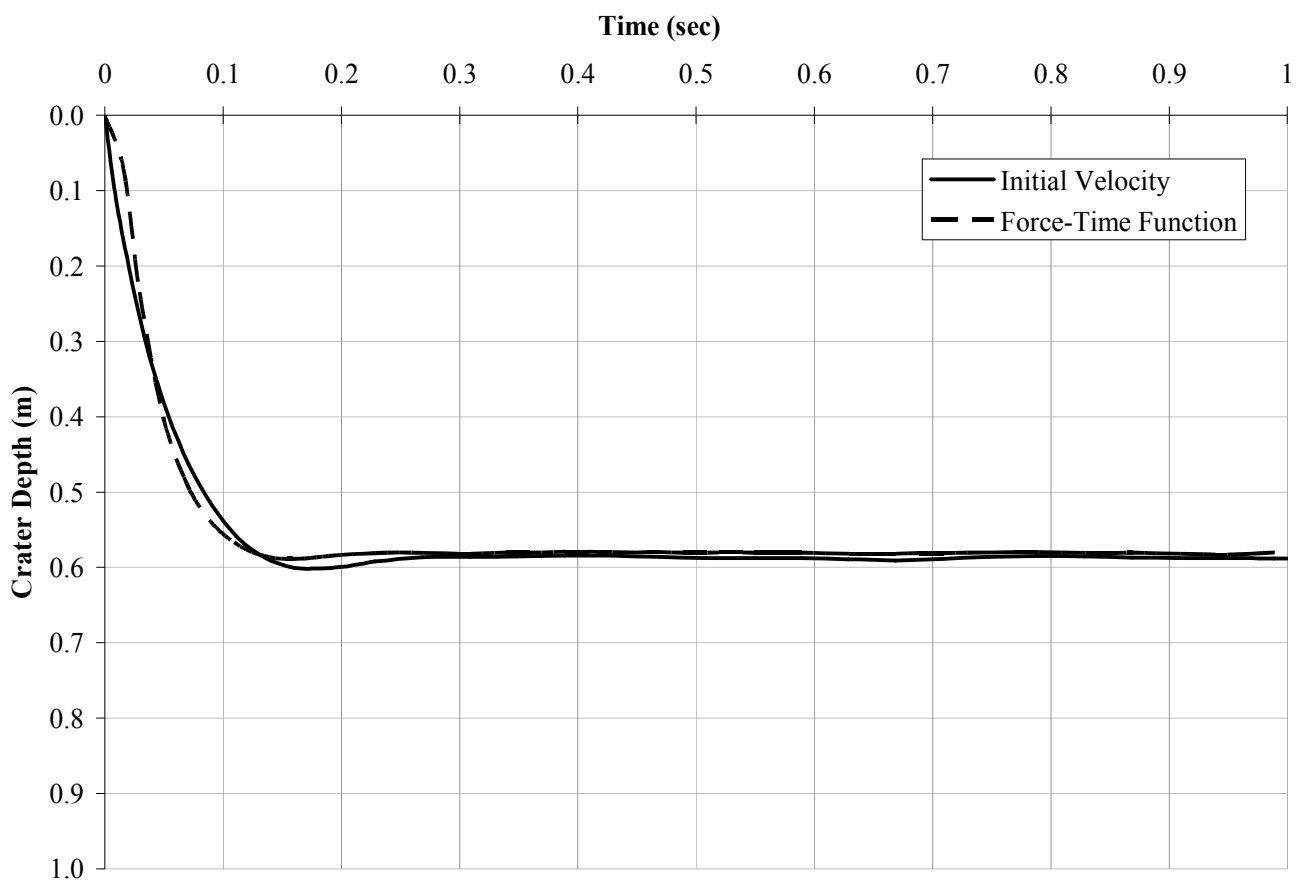

Figure 3-6 Crater depth vs. time 


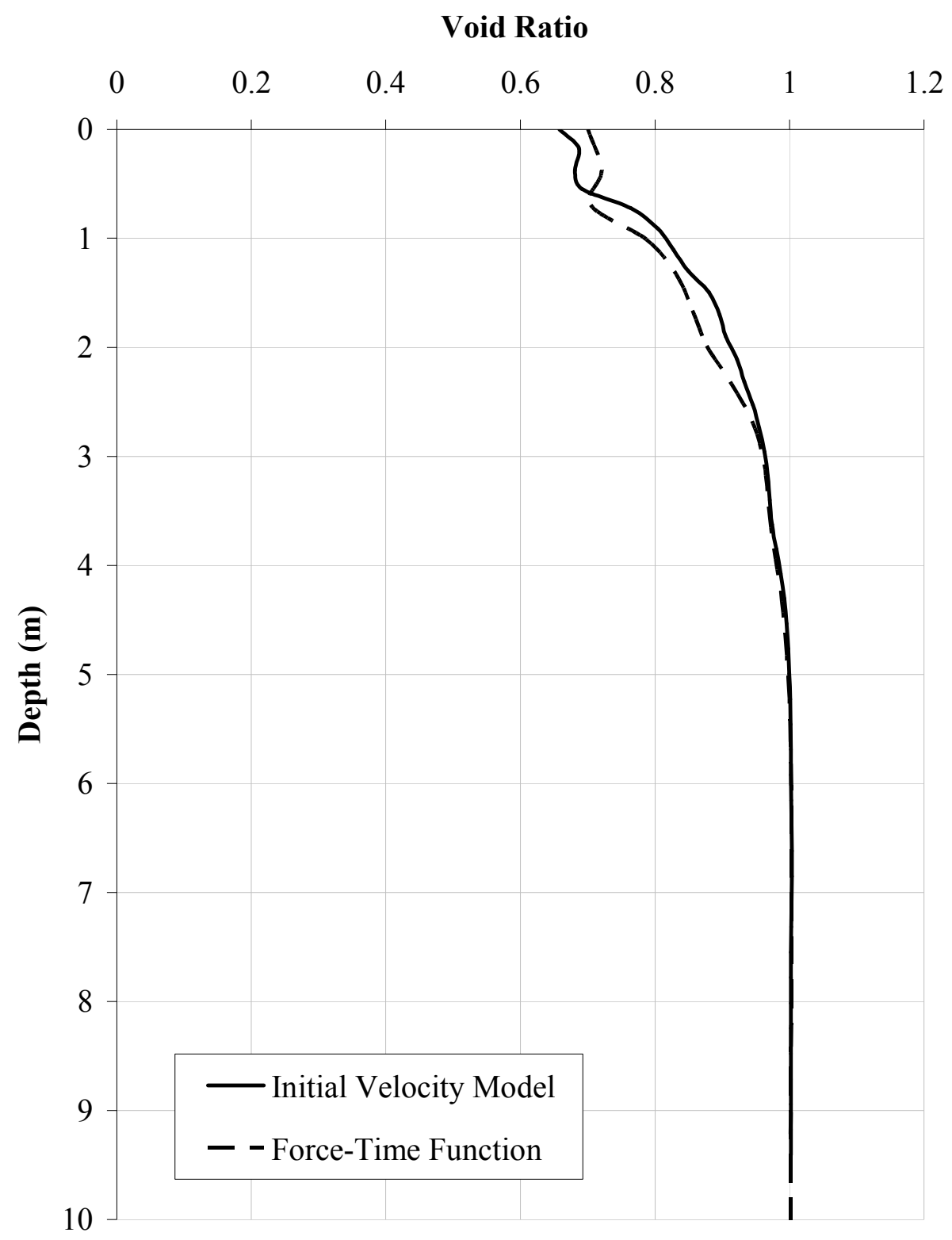

Figure 3-7 Comparison of void ratio distribution

\subsubsection{Improving Numerical Performance of Multiple Drops Model}

The force-time FE model was used for modeling multiple tamping. Six analysis steps (dynamic/implicit) was used to model the multiple drops. At each step, pressure was applied. The modeling simulation for each step lasts for 1 second which is 
approximately enough for the surface waves to reach the boundaries. The first trial of the model shows that there were severe deformations around the tamper, thus resulting in highly distorted elements. Figure 3-8 shows the void ratio distribution after the second drop. It can be noticed that the element around the tamper suffer excessive deformations which prohibited simulation for more than two drops.

It is not possible to avoid the large deformations that occur during compaction, especially with multiple drops case in which the crater depth ranges from 1 to 1.5 meters. Therefore, a simple technique was proposed to avoid severe element distortion. A groove with 0.2 meter width and 0.15 meter depth is placed around the peripheral of the tamper. Figure 3-9 shows the location and dimensions of the proposed groove.

Simulation results for multiple drops are shown in Figure 3-10 and Figure 3-11 for drops 1 to 3 and drops 4 to 6 , respectively. The distribution of void ratio shows three clearly identified zones. The first zone is the zone of treatment in which void ratio is less than the initial void ratio. Vertical and lateral extent of treatment are noticed and found propagating vertically and horizontally with the sequential drops. The second zone is located above the crater base around the peripheral of the tamper. In this zone, increase in void ratio is noticed. Also, heave is formed around the tamper which has the highest increase in void ratio. The third and final zone is the zone in which no change in initial void ratio is noticed. From Figure 3-10 and Figure 3-11, it may be concluded that the simulation results of multiple drops model look realistic. The validation for FE models is presented in the following section. 


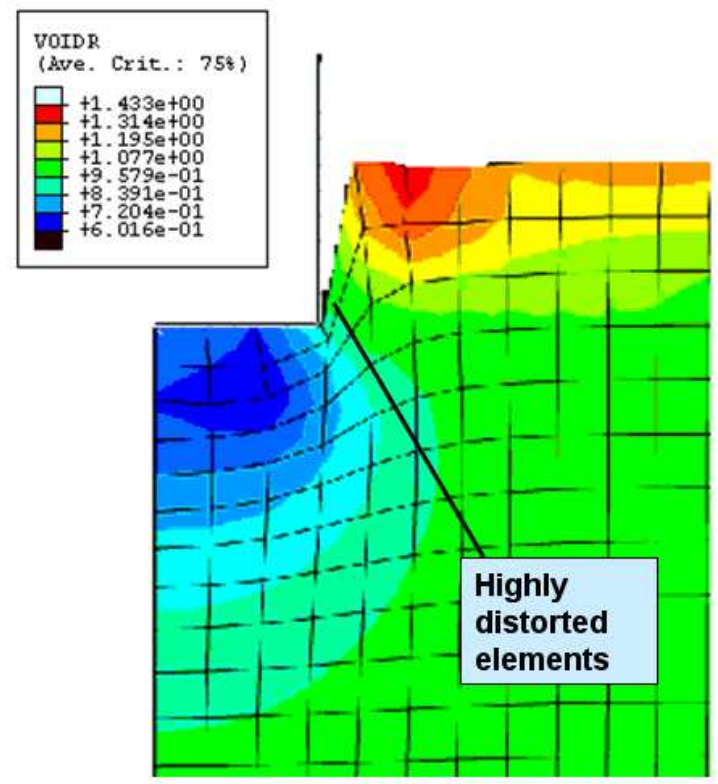

Figure 3-8 Excessive deformation after the second drop

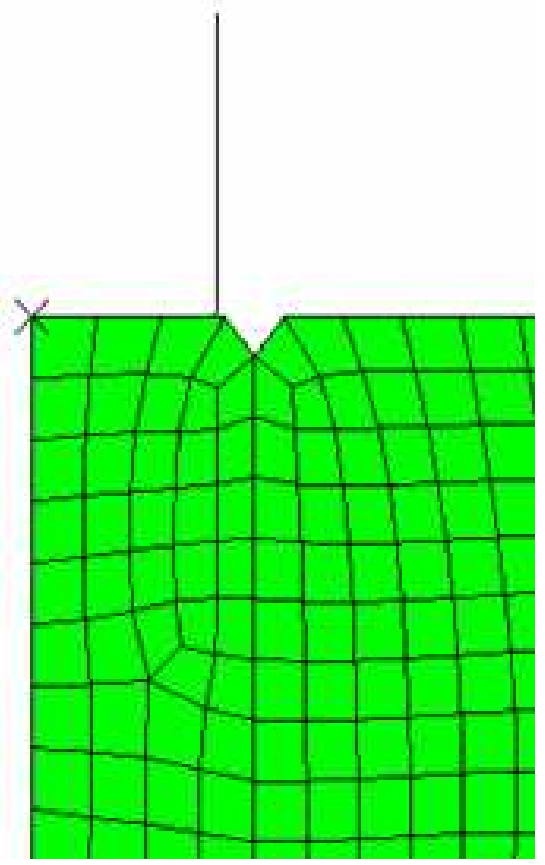

Figure 3-9 Groove location and element distribution for the proposed solution 


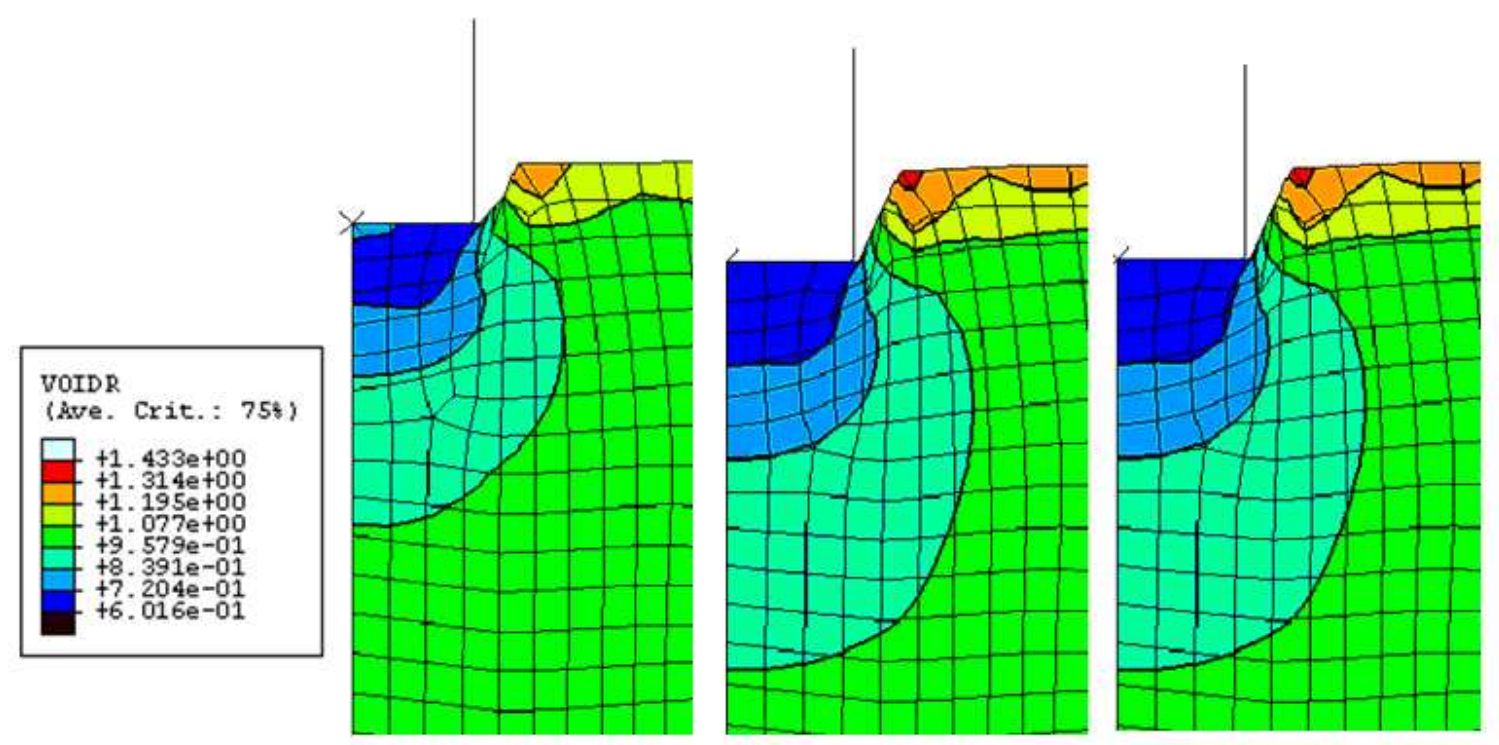

Figure 3-10 Void ratio distribution for improved numerical performance (drops 1 to 3 )
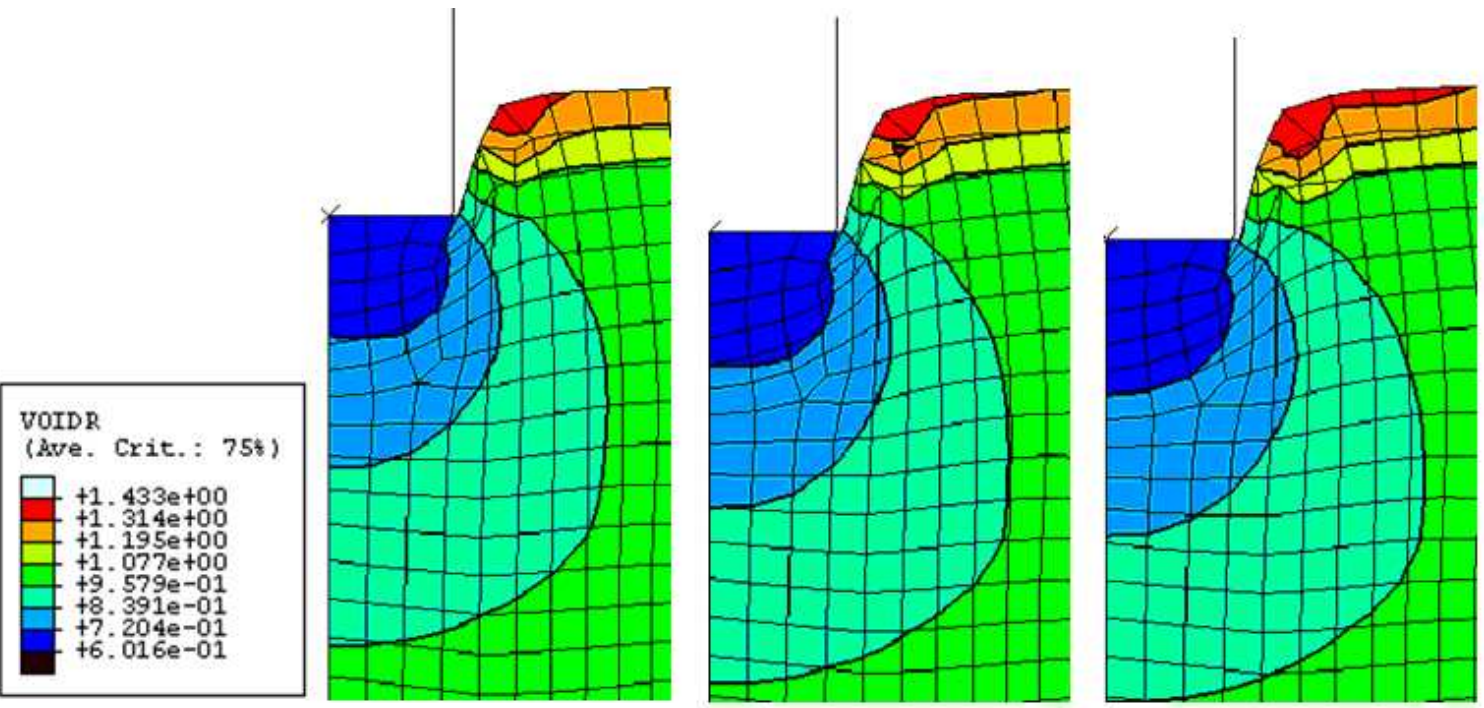

Figure 3-11 Void ratio distribution for improved numerical performance (drops 3 to 6 )

\subsection{Model Validation}

In this section, FE model is validated using in-situ measurements published in open literature. Since the data for dynamic compaction in clays are rare in the literature and most of the practical design models were based on data collected from sands sites; 
therefore, the same FE model geometry was used to conduct simulation on sand. The constitutive behavior of sand was modeled using Mohr-Coulomb plasticity model. The following sub-sections show the results for both clays and sands validations.

\subsubsection{Validation of FE Model with Cam-Clay Constitutive Model}

Mayne (1984) summarized the results for DC operation that were conducted for 120 sites. Figure 3-12 shows the variation of crater depth with the normalized tamping energy for different soil types. The horizontal axis represents the tamping energy normalized to the tamper cross sectional area. It can be noticed the influence of soil type on the crater depth magnitude. Even with the same soil type, sand for example, the induced crater depth falls in a wide range.

Several FE simulations were conducted on the baseline model with Cam-Clay constitutive model using different tamping energies. Data collected from Figure 3-13 (represented by the intersected of vertical and horizontal dashed lines) were compared to the simulation results and plotted in Figure 3-13. It can be seen that crater depth obtained from FE simulation falls in the range compared to in-situ measured data. It is important to mention that exact match may not be achieved since FE simulations are based on specific soil properties and soil profile which may not match with what Mayne (1984) studied. Based on those comments, the influence of initial soil properties on crater depth was taken as the main concern for the work presented in this dissertation. 


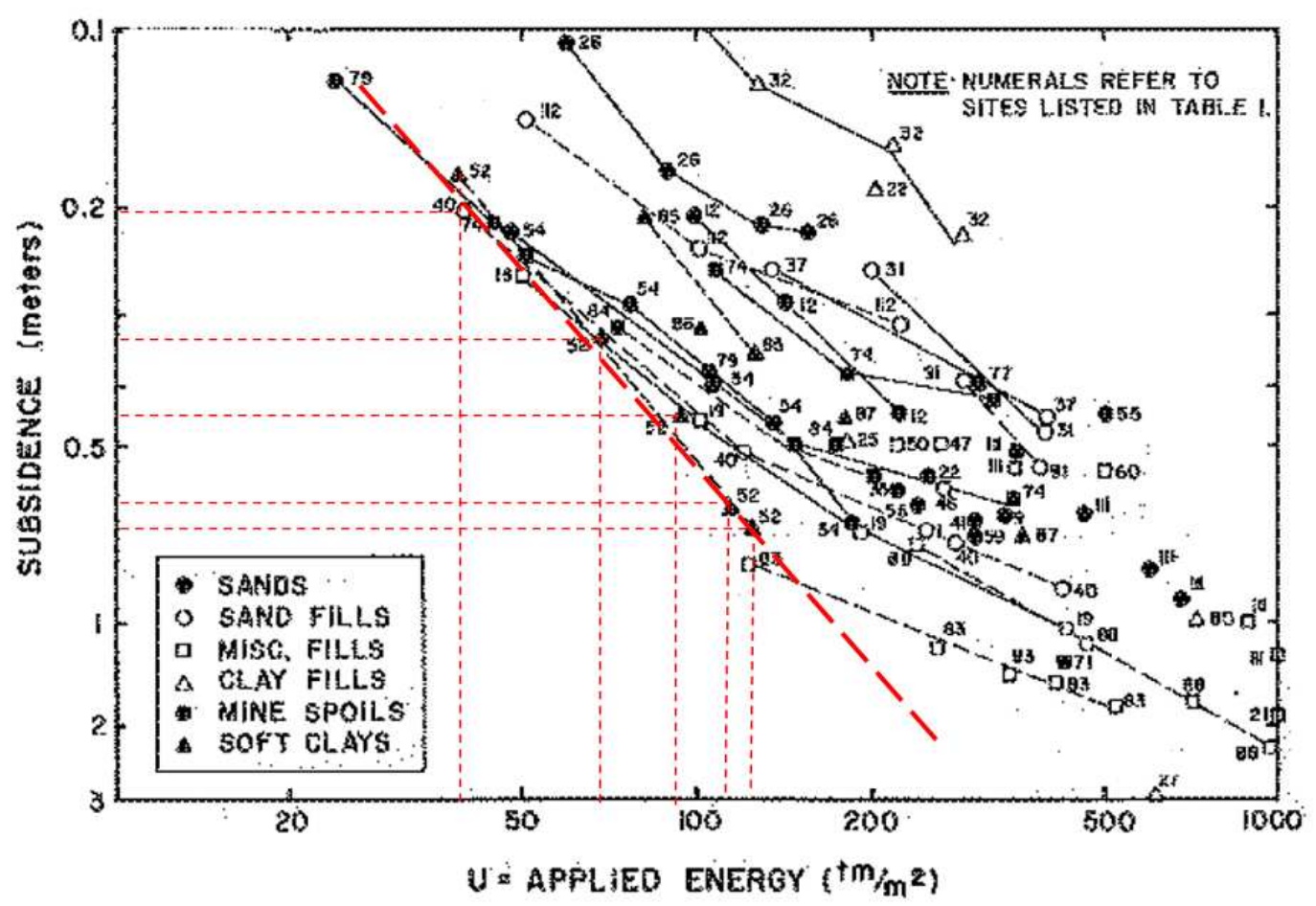

Figure 3-12 Crater depth for different soil types (Mayne ,1984)

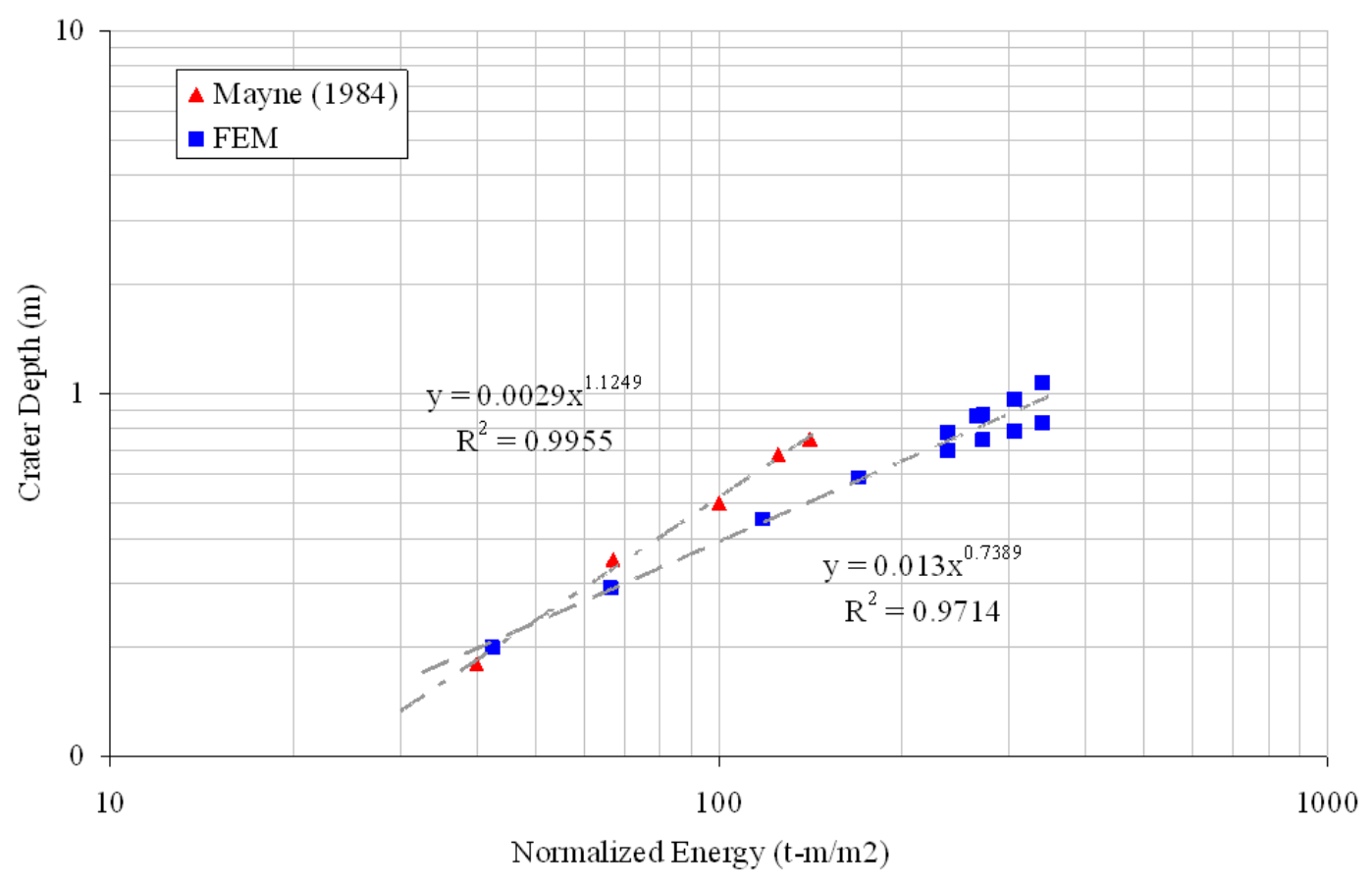

Figure 3-13Validation of crater depth (Clay data) 


\subsubsection{Verification Using Sand Model}

Design models for dynamic compaction that were presented in the literature were mainly derived based on data collected from mostly sand sites. Therefore, the FE model presented in the earlier section was modified to model the compaction process for sands. Based on literature review of the sand data, which were shown in Table 3-3 and

Table 3-4, parameters for Mohr-Coulomb constitutive model for sand were selected and listed in Table 3-5.

The FE model for sand was used to simulate tamping with different tamping energies. While the data of sand in Mayne (1984) showed a wide range for the crater depth, the group of points that were found to fall in the same range (represented by the intersected vertical and horizontal dashed lines) was plotted on Figure 3-15. It can be noticed that crater depth falls in the same range. Again, as stated earlier for Cam-Clay model, the exact match may not be achieved since FE simulations are based on specific soil properties and soil profile which may not match with what Mayne (1984) studied.

Table 3-3 Properties of sands (Peck 1974)

\begin{tabular}{c|c|c}
\hline $\begin{array}{c}\text { SPT Penetration, N-Value } \\
\text { (blows/ foot) }\end{array}$ & $\begin{array}{c}\text { Density } \\
\text { of Sand }\end{array}$ & $\begin{array}{c}\text { Friction Angle } \\
\text { (degrees) }\end{array}$ \\
\hline$<4$ & Very loose & $<29$ \\
\hline $4-10$ & Loose & $29-30$ \\
\hline $10-30$ & Medium & $30-36$ \\
\hline $30-50$ & Dense & $36-41$ \\
\hline$>50$ & Very dense & $>41$ \\
\hline
\end{tabular}


Table 3-4 Young's modulus of sands (Peck 1974)

\begin{tabular}{l|c}
\hline \multicolumn{1}{c|}{ Soil } & $\mathrm{E}_{\mathrm{s}}(\mathrm{tsf})$ \\
\hline Loose sand & $100-250$ \\
\hline Dense sand & $250-1000$ \\
\hline Dense sand and gravel & $1000-2000$ \\
\hline Silty sand & $250-2000$ \\
\hline
\end{tabular}

Table 3-5 Material properties for sand model

\begin{tabular}{l|c}
\hline \multicolumn{1}{c|}{ Soil Property } & Value \\
\hline Friction angle & 30 \\
\hline Young's Modulus $(\mathrm{MPa})$ & 15 \\
\hline Density $\left(\mathrm{kg} / \mathrm{m}^{3}\right)$ & 1800 \\
\hline Passion's Ratio & 0.25 \\
\hline
\end{tabular}

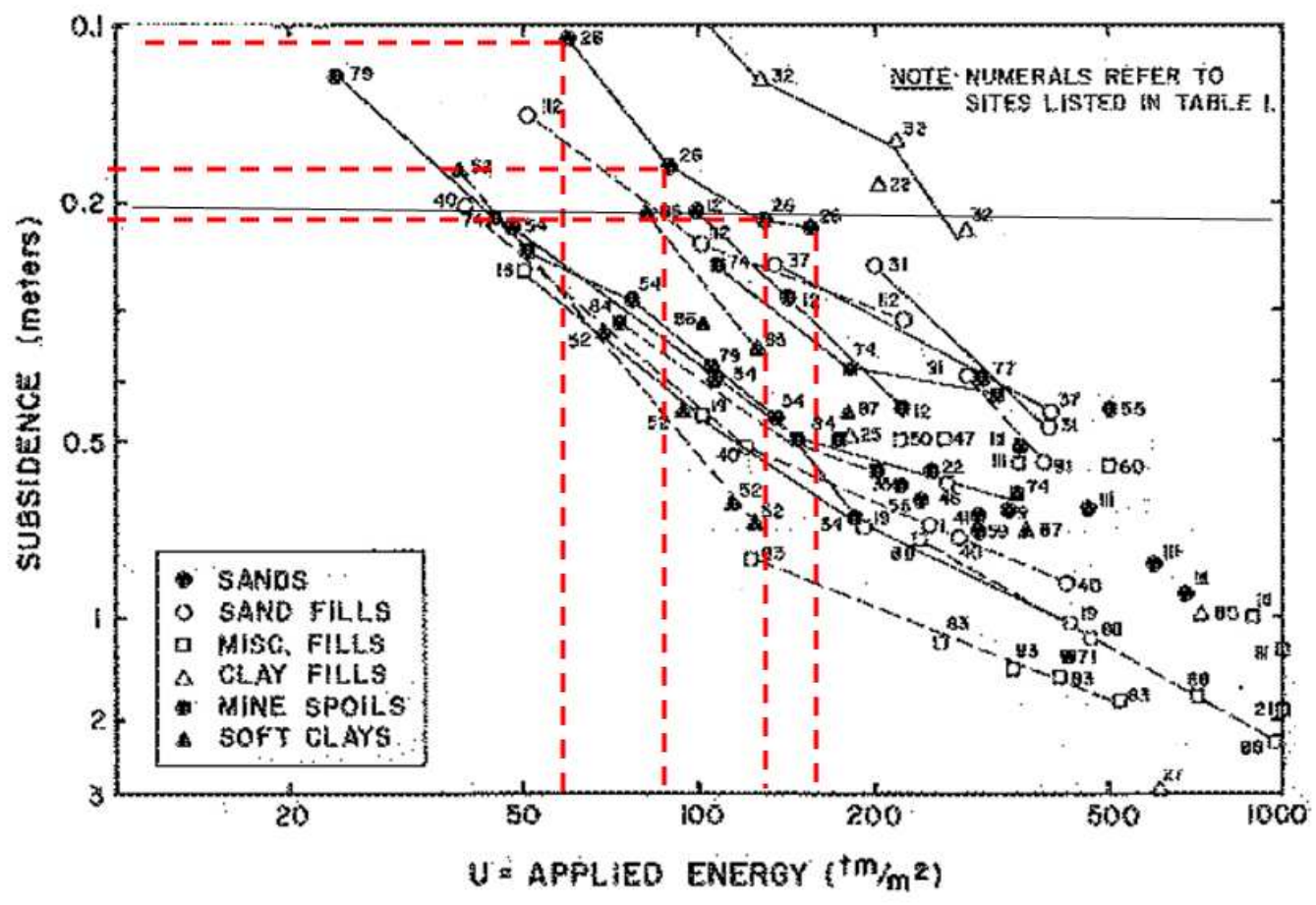

Figure 3-14 Sand Data (Mayne, 1985) 


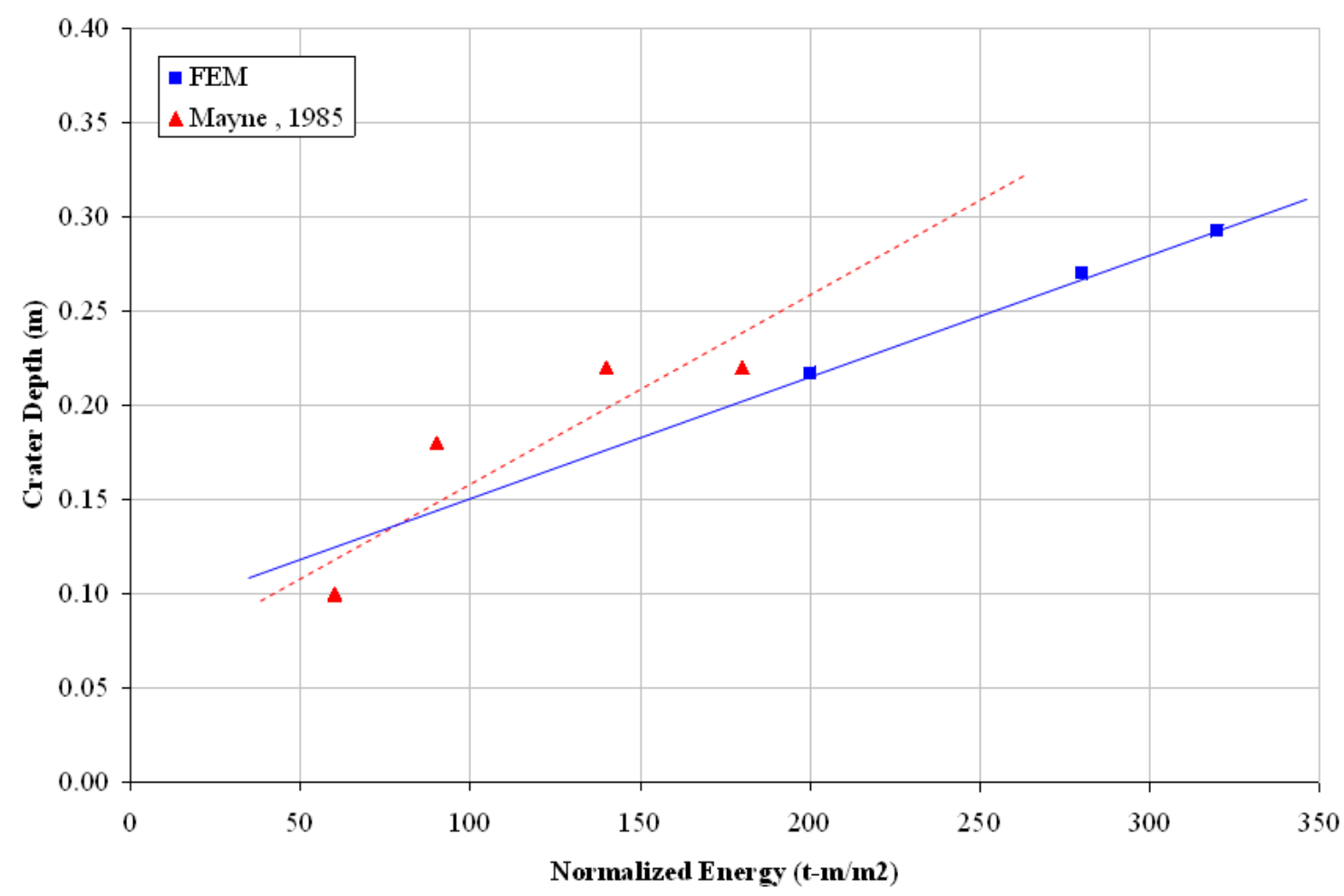

Figure 3-15 Validation of crater depth (sand model)

Validation of the ability of FE model to estimate the PPV was of concern. Mayne derived correlations to estimate Peak Particle Velocity (PPV) based on in-situ measurements, as listed in section 2-8. The results obtained from FE sand model are presented and compared to Mayne models in Figure 3-16. The horizontal axis represents the scaled energy. The scaled energy $\sqrt{W H} / d$ value usually ranges from 0.2 to 2 . Assuming 20 tons tamper is dropped from 20 meters height, the scaled energy evaluated at 20 meters distance far from the tampering center is equal to 1.0. Also, if a 10 tons tamper is dropped from 10 meters height, the scaled energy is equal to 0.5 when evaluated at 20 meters. 


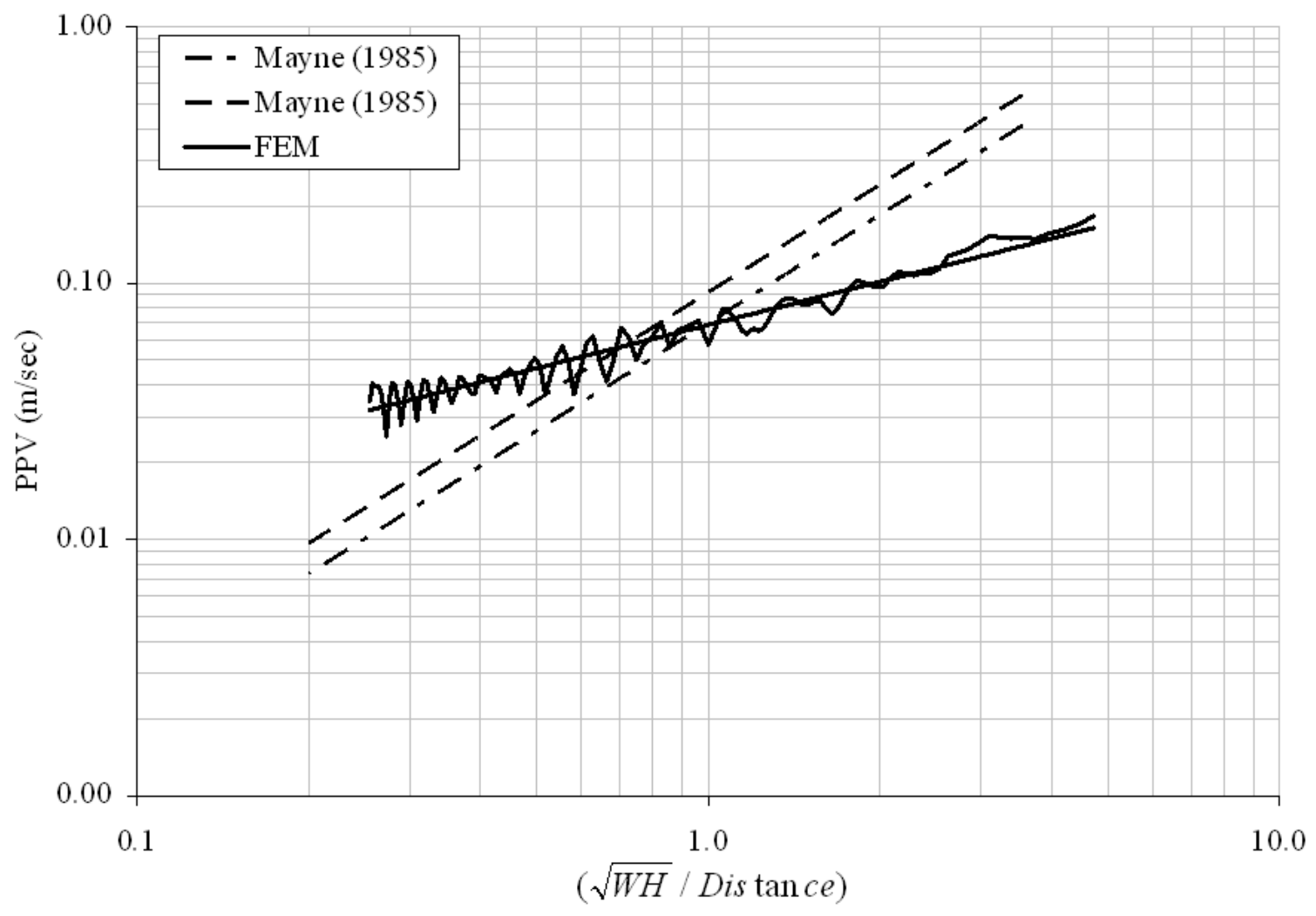

Figure 3-16 Validation of peak particle velocity

\subsection{Summary and Conclusions}

In this chapter, FE models were developed to model dynamic compaction in cohesive and cohesionless soils. Soil continuum was modeled using axi-symmetric elements. The boundary of the model was modeled using infinite element to minimize reflection from the boundaries. A geostatic step (analysis) was used to define the initial state stress that exists in the soil. The dynamic effect of tamping was modeled using Dynamic/Implicit analysis.

Two models were developed to model single drop and multiple drops. In the first model concentrated mass with initial velocity was used to model the tamper striking soil surface. An equivalent force-time function model was used to model multiple drops case 
in which 6 consecutive drops could be modeled successfully. Additional modifications involving the use of artificial groove for the multiple drops model were essential to model the large deformation associated with multiple drops model.

The FE models were validated using field data that are available in the literature. Both Cam-Clay model and Mohr-Coulomb model for clay and sand, respectively, were evaluated. A good match could be achieved between field measurements and FE models results for cohesive and cohesionless soils.

The following summarizes the contribution of the presented work compared to the work presented in the literature are summarized as followings.

- A full state of stress was modeled using the built-in analysis option in ABAQUS FE code (Geo-Static) which calculates the horizontal and vertical stresses.

- Cam-Clay constitutive model was used to describe soil behavior in which the highly non-linear stress-strain behavior of soils could be captured.

- Two models were developed to model single and multiple drops. The models were validated using actual field measurements of sand and soft clay cases.

The following observations can be made regarding the field data used for the validation

- Even for the same soil type (e.g., sands) the induced crater depth falls in a wide range for the same normalized energy. This indicates that soil parameters are important influencing factor, in addition to soil type. 
- Information about soil properties and soil profile before compaction was not provided in the reported data, thus making empirical equations for qualitative estimate.

- The FE models were considered to be accurate if the results falls in the range of the values obtained from the literature.

- Mohr-Coulomb model was successfully used to describe stress-strain behavior of cohesionless soils. 


\section{CHAPTER IV}

\section{MODELING RESULTS OF DYNAMIC COMPACTION IN COHESIVE SOILS}

\subsection{Introduction}

In this chapter a parametric study was carried out using the FE model that was presented and validated in Chapter III. Parametric study was conducted using ABAQUS FE code to investigate the influence of tamping energy as well as Cam-Clay constitutive model parameters on the depth of the induced crater and the dimensions of zone of influence. In addition, variation of Peak Particle Velocity (PPV) with soil properties and tamping energy was investigated. Finally, analysis for the deceleration time function of the tamper mass was carried out to obtain the frequency range of the associated waves.

\subsection{Parametric Study}

An extensive parametric study using ABAQUS FE Code and the modeling technique developed in Chapter III was carried out to examine systematically the effects of several influencing factors on DC phenomenon in cohesive soils. The pertinent CamClay model parameters were reviewed in Chapter III, which include the following set $\left(\rho, v, M, e_{o}, \lambda, a_{o}\right)$. The definition of these six Cam-Clay model parameters was provided previously in chapter III. The logarithmic elastic bulk modulus $(k)$ was excluded in the parametric study as it was assumed to equal one tenth of the logarithmic plastic bulk 
modulus $(0.1 \lambda)$. The tamper parameters in the parametric study include tamper radius $(R)$, tamper mass $(m)$, and tamper drop height $(H)$. Based on intensive literature review presented in Chapter II, the range for each parameter was selected and summarized in Table 4-1 and Table 4-2 for tamper parameters and Cam-Clay model parameters, respectively.

Table 4-1 Tamper parameters for parametric study

\begin{tabular}{l|c}
\hline Parameter & Range \\
\hline Mass (tons) & $10-20$ \\
\hline Drop height (m) & $10-20$ \\
\hline Mass radius (m) & $1-2.5$ \\
\hline
\end{tabular}

Table 4-2 Cam clay model parameters for FE parametric study

\begin{tabular}{l|l|c|c}
\hline Parameter & Symbol & Base Line Value & Range \\
\hline Soil density & $\rho(\mathrm{kg} / \mathrm{m} 3)$ & 2000 & $1500-2500$ \\
\hline Poisson's ratio & $v$ & 0.25 & $0.15-0.35$ \\
\hline Logarithmic plastic bulk modulus & $\lambda$ & 0.20 & $0.04-0.4$ \\
\hline Logarithmic elastic bulk modulus & $\kappa$ & 0.02 & $0.004-0.04$ \\
\hline Initial yield surface & $a_{0}(\mathrm{kPa})$ & 50 & $30-70$ \\
\hline Critical state line slope & $M$ & 1.00 & $0.8-1.2$ \\
\hline Initial void ratio & $e_{0}$ & 1.0 & $0.50-1.25$ \\
\hline
\end{tabular}

* Baseline value is the reference value in the parametric study, when only one parameter is varied while keeping other parameters at the baseline value.

\subsection{Crater Depth}

Crater results from the impact of the falling tamper onto the ground surface. Usually craters are surrounded by a heave of soil. The crater depth is considered as a measure for site suitability for DC in terms of the degree of improvement achieved. According to Oshima (1994), crater depth depends on the normalized tamper momentum (per unit area) and proportional to the square root of number of drops. 
The influence of tamping energy on the variation of crater depth can be studied by analyzing the simulation results in which soil properties were kept constant while changing tamping energy. The soil properties for this group of simulation are listed as base line properties in Table 4-2. Figure 4-1 shows the variation of crater depth with striking velocity. A linear correlation can be used for correlation. The goodness of fit was examined using R-squared which was found to be equal to 0.9997 . It should be mentioned that the horizontal axis in Figure 4-1 represents the striking velocity which is calculated using Newton's Law. The striking velocity for the free falling mass can be calculated as $V_{o}=\sqrt{2 g H}$; where $\mathrm{g}$ is the ground acceleration and $H$ is the drop height. Five drops heights were selected 10,14, 16, 18 and 20 meters which correspond to striking velocity equal to $14,16.5,17.7,18.8$, and $20(\mathrm{~m} / \mathrm{sec})$, respectively. A ten tons mass tamper with 1.25 meter in radius were used for this group of simulations.

Figure 4-2 shows the variation of the crater depth with the mass tamper. The same procedure for developing Figure 4-1 was used. The base line properties listed in Table 4-2 were kept constant for this group of simulations. Five tamper masses were selected 10,14,16,18 and 20 tons. The five masses were dropped from 10 meters height which correspond to striking velocity equal to $14(\mathrm{~m} / \mathrm{sec})$. A linear correlation can be used to describe the variation of crater depth with tamper mass. The goodness of fit was also examined using R-squared which was found to be equal to 0.9803 .

The variation of crater depth with tamper area is shown in Figure 4-3. Increasing the tamper radius results in reduction in tamper energy density; hence, the expected reduction in crater depth. Five tamper radii were used 1, 1.25, 1.75, 2, and 2.5 meters. This corresponds to tamper area equal to $3.2,4.9,9.6,12.5$, and $19.6 \mathrm{~m}^{2}$, respectively. 
Similarly, the base line properties listed in Table 4-2 were kept constant for this group of simulations. Also, tamper mass and drop height were kept constant and equal to 10 tons and 10 meter, respectively. Crater depth is inversely proportional to the tamper area. The goodness of fit was also examined using R-squared which was found to be equal to 0.9992 .

Figure 4-4 shows the variation of crater depth with the number of drops. The multiple drops model was developed using the base line properties listed in Table 4-2. The tamper energy for this model is equal to 10 tons mass dropped from 10 meters height. The crater depth is proportional to the square root of number of drops. Similarity, goodness of fit was also examined using R-squared which was found to be equal to 0.9926 .

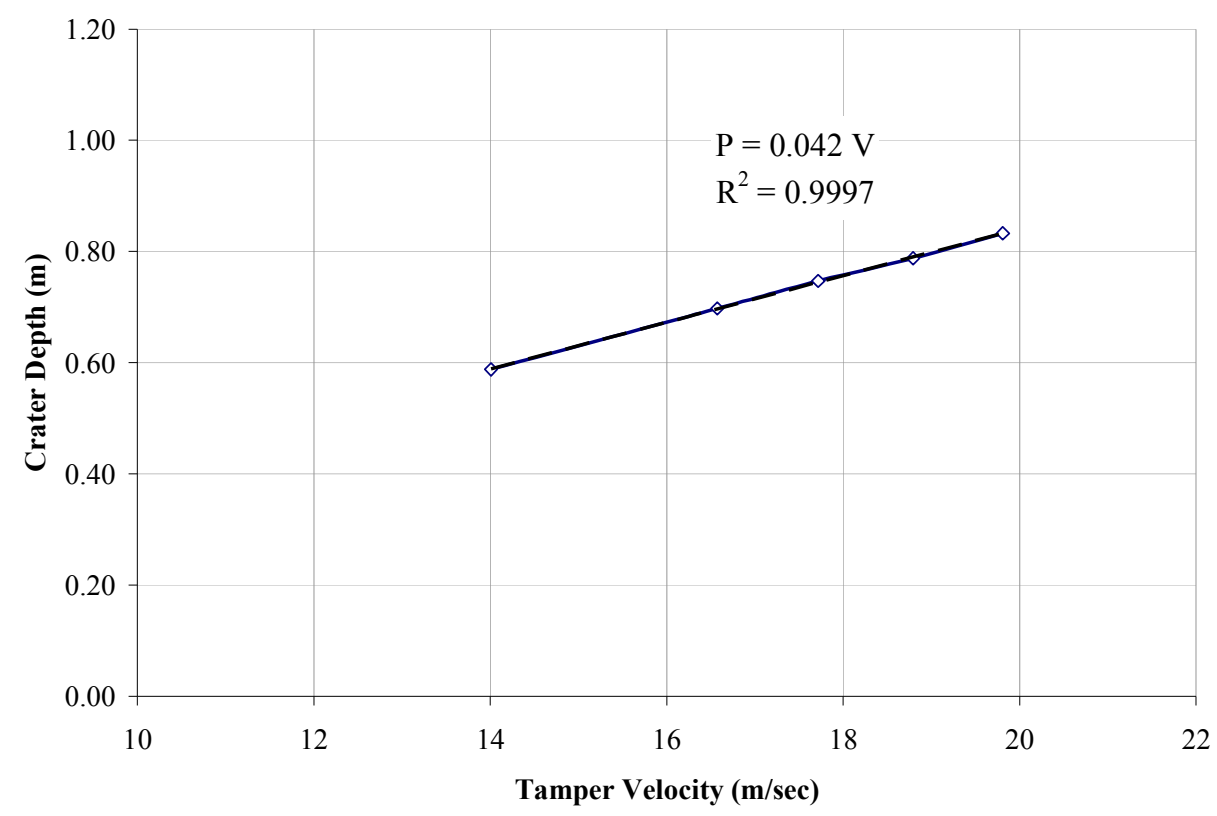

Figure 4-1 Variation of crater depth with tamper velocity 


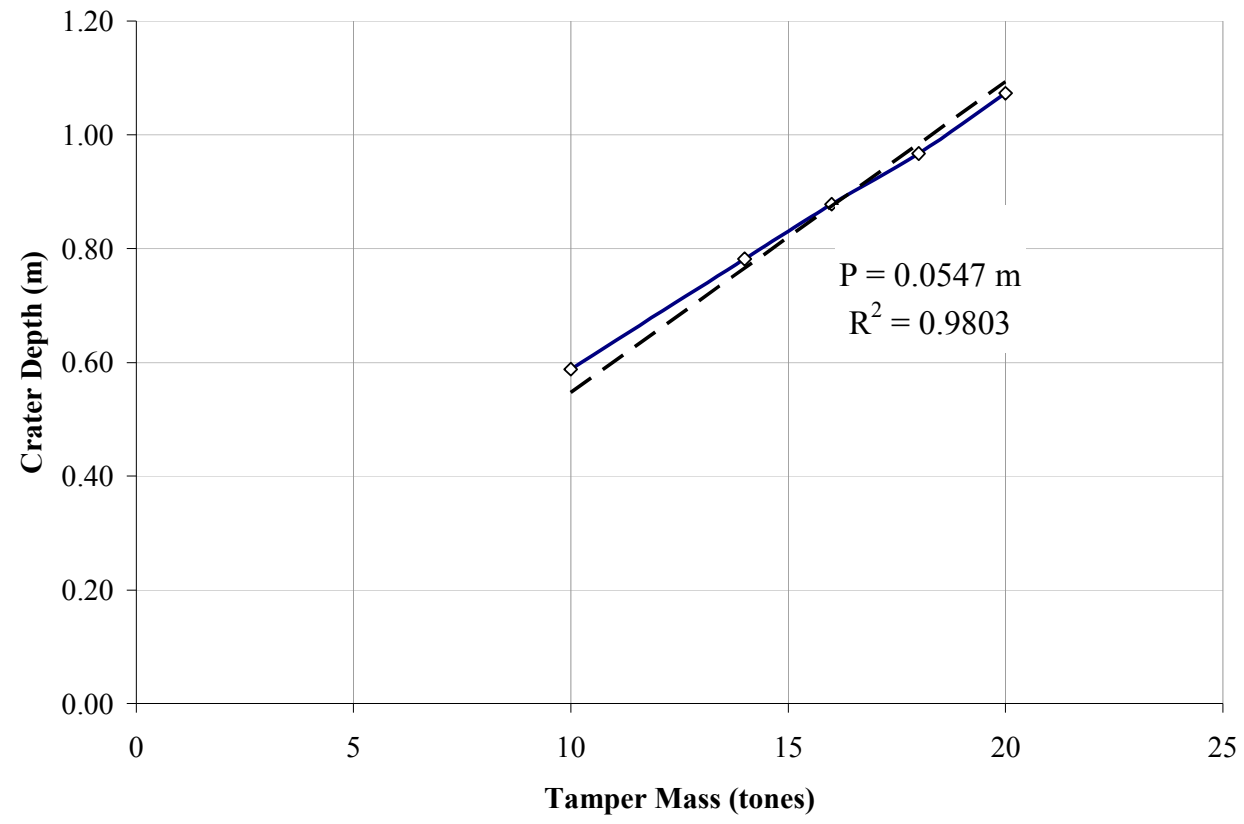

Figure 4-2 Variation of crater depth with tamper mass

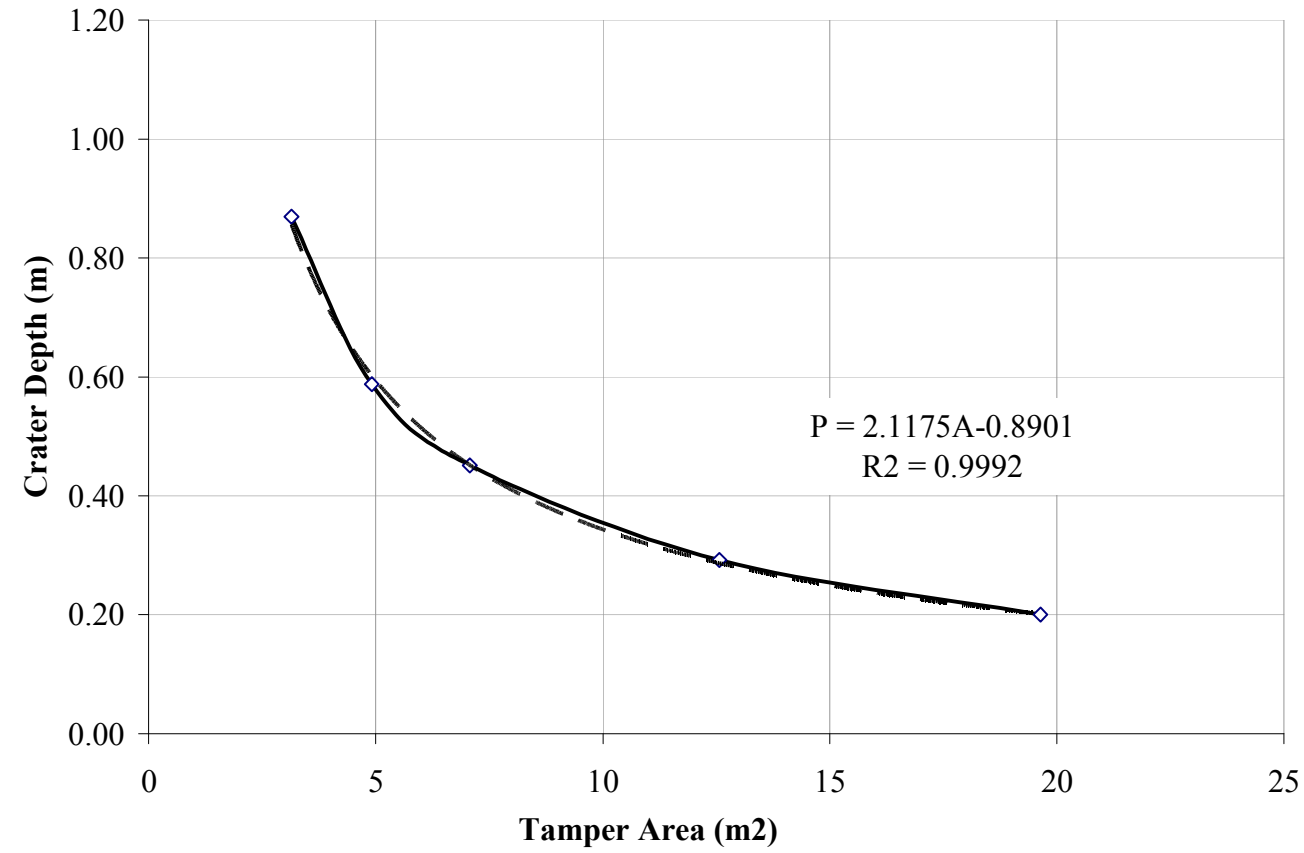

Figure 4-3 Variation of crater depth with tamper area 


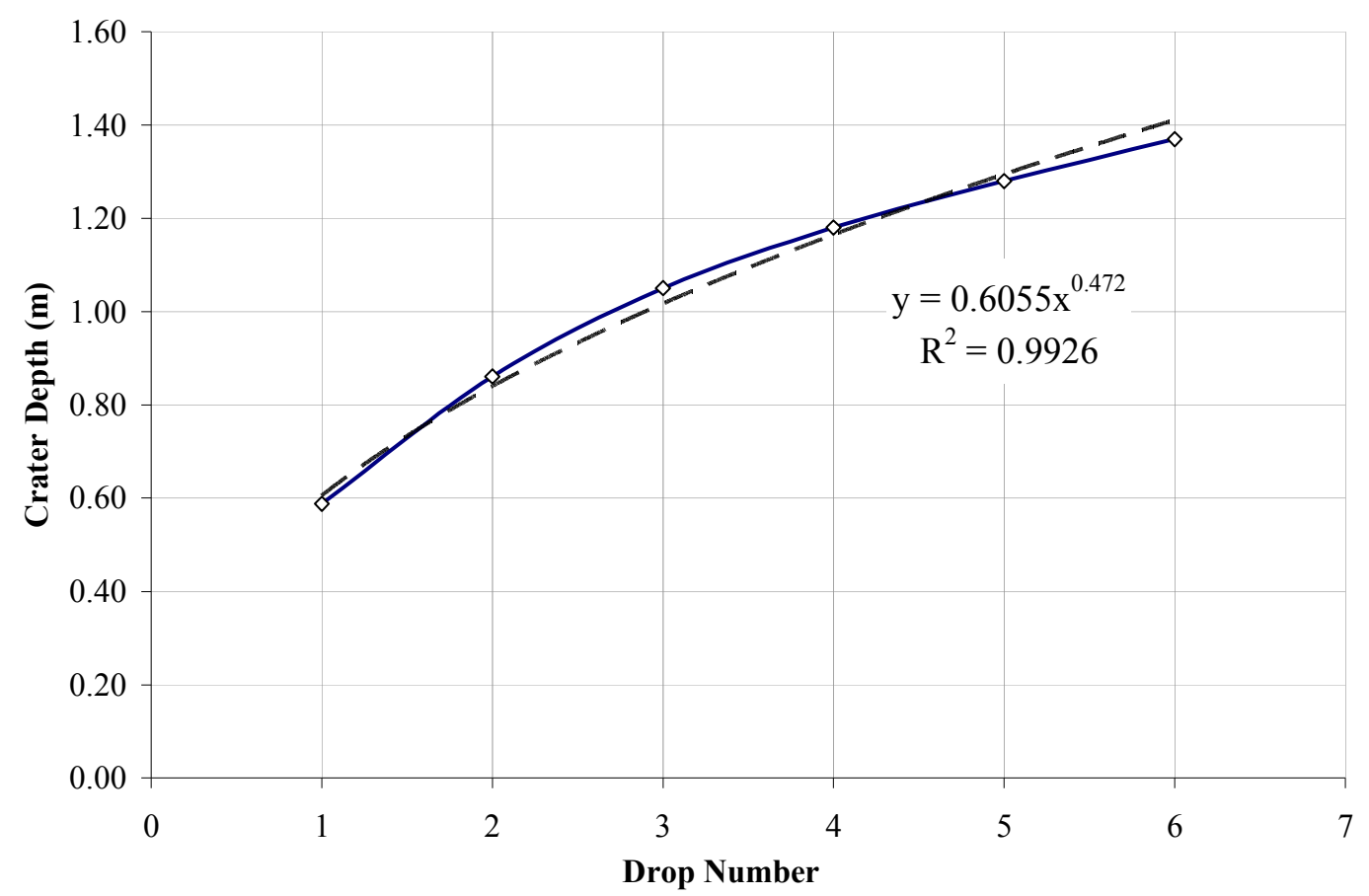

Figure 4-4 Effect of multiple drops on crater depth

From the previous discussion, Equation (4-1) can be used to describe the variation of the crater depth with the tamper characteristics (mass, velocity, and area) and the total number of drops. A parameter $\mathrm{C}$ was used as proportional constant. The sensitivity of parameter $\mathrm{C}$ to soil properties will be investigated in this section. Figure 4-5 shows the variation of crater depth with the normalized tamper momentum, per tamper area. A linear correlation can be used and Equation (4-1) is proposed to represent the variation of carter depth with ram momentum and number of drops. The goodness of fit was examined using R-squared which was found to be equal to 0.9706 .

$P=C\left(\frac{m V_{0}}{A}\right) \sqrt{N}$

Where

$\mathcal{C}:$ Parameter depends on soil properties 


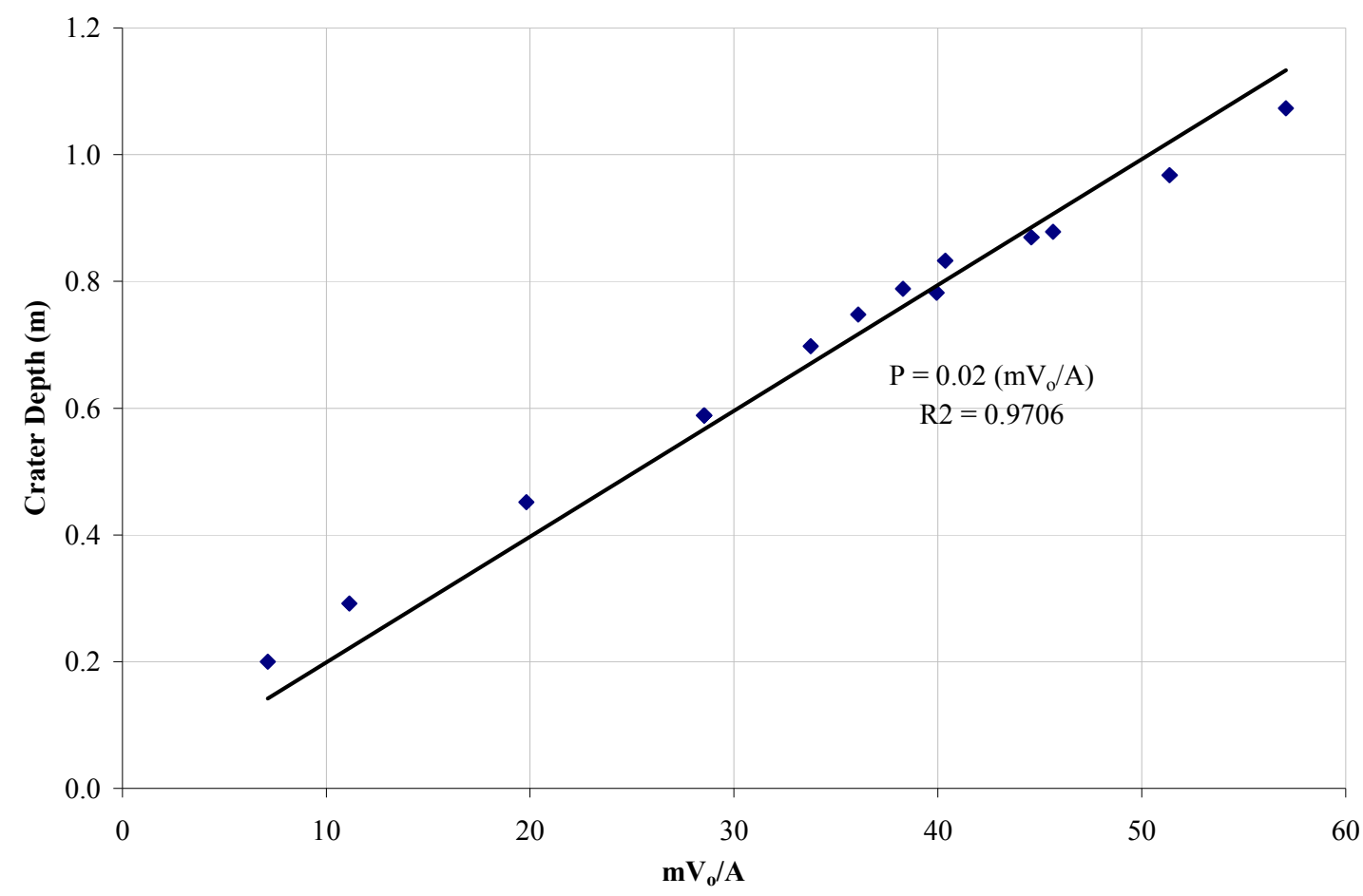

Figure 4-5 Effect of tamper momentum on crater depth due to single drop

The parametric study conduced in this chapter also considers the variation of soil properties and their influence on crater depth. For each simulation in this study, tamping energy was kept constant and equal to 10 tons dropped from 10 meter height. Soil properties were also kept constant and equal to the base line properties listed in Table 4-2 except the soil property which was the subject of study.

Figure 4-6 show the variation of crater depth with initial soil density. Five densities were used for this model 1500, 1750, 2000, 2250, and $2500\left(\mathrm{~kg} / \mathrm{m}^{3}\right)$. Decrease in crater depth is noticed with increasing initial soil density. Increasing soil density results in increase in soil strength hence high resistance to DC. A power function was used for correlation and the goodness of fit was examined using R-squared which was found to be equal to 0.9988 . 
The influence of pre-consolidation pressure is investigated utilizing the same procedure of the parametric study, changing soil property one at a time while keeping tamping energy and the rest of soil properties constant. Similarly, five values for the preconsolidation pressure were selected $60,80,100,120$ and $140 \mathrm{KPa}$. The crater depth in Figure 4-7 is plotted versus the parameter $a_{o}$ which is the radius of the initial yield surface and equal to half the value of the pre-consolidation pressure. The preconsolidation pressure indicates the level of stress to which the soil was exposed before compaction. Therefore, high pre-consolidation pressure results in high resistance for DC. A power function was used for correlation and the goodness of fit was examined using Rsquared which was found to be equal to 0.994 .

Figure 4-8 show the variation of crater depth with the slope of Critical State Line (CSL) which is defined as parameter M. Five values for the Critical Slope Line (CSL) slope were selected $0.8,0.9,1.0,1.1$, and 1.20 . Increasing the slop of CSL resulted in decrease in crater depth. Again, a power function was used for correlation and the goodness of fit was examined using R-squared which was found to be equal to 0.9974 .

Figure 4-9 show the variation of crater depth with the logarithmic plasticity bulk modulus $(\lambda)$. As was previously mentioned, $\lambda$ is the slope of the normal consolidation line (ncl) in the $\left(\ln \left(p^{\prime}\right)-e\right)$ plan; where $p^{\prime}$ is the mean effective stress and $e$ is the void ratio. Increasing the slope of ncl will result relatively large change in the void ratio for the same applied mean effective stress. Therefore, increasing $\lambda$ resulted in significant increase in crater depth. Five points were selected for the simulation 0.04, 0.1, 0.2, 0.3 
and 0.4. Following the same procedure, a power function was used for correlation and the goodness of fit was examined using R-squared which was found to be equal to 0.9992 .

The variation of crater depth with Poisson's ratio is shown in Figure 4-10. Five points were selected $0.15,0.2,0.25,0.30$ and 0.35 . The crater depth was found be insensitive to variations to Poisson's ratio. Finally, the variation of crater depth with initial void ratio is presented in Figure 4-11. Soils with relatively high initial void ratio are highly suitable for DC. Therefore, significant increase in crater depth is noticed as a result of increasing initial void ratio. Four points were used $0.65,1.0,1.15$ and 1.25. A power function was used for correlation and the goodness of fit was examined using Rsquared which was found to be equal to 0.9873 .

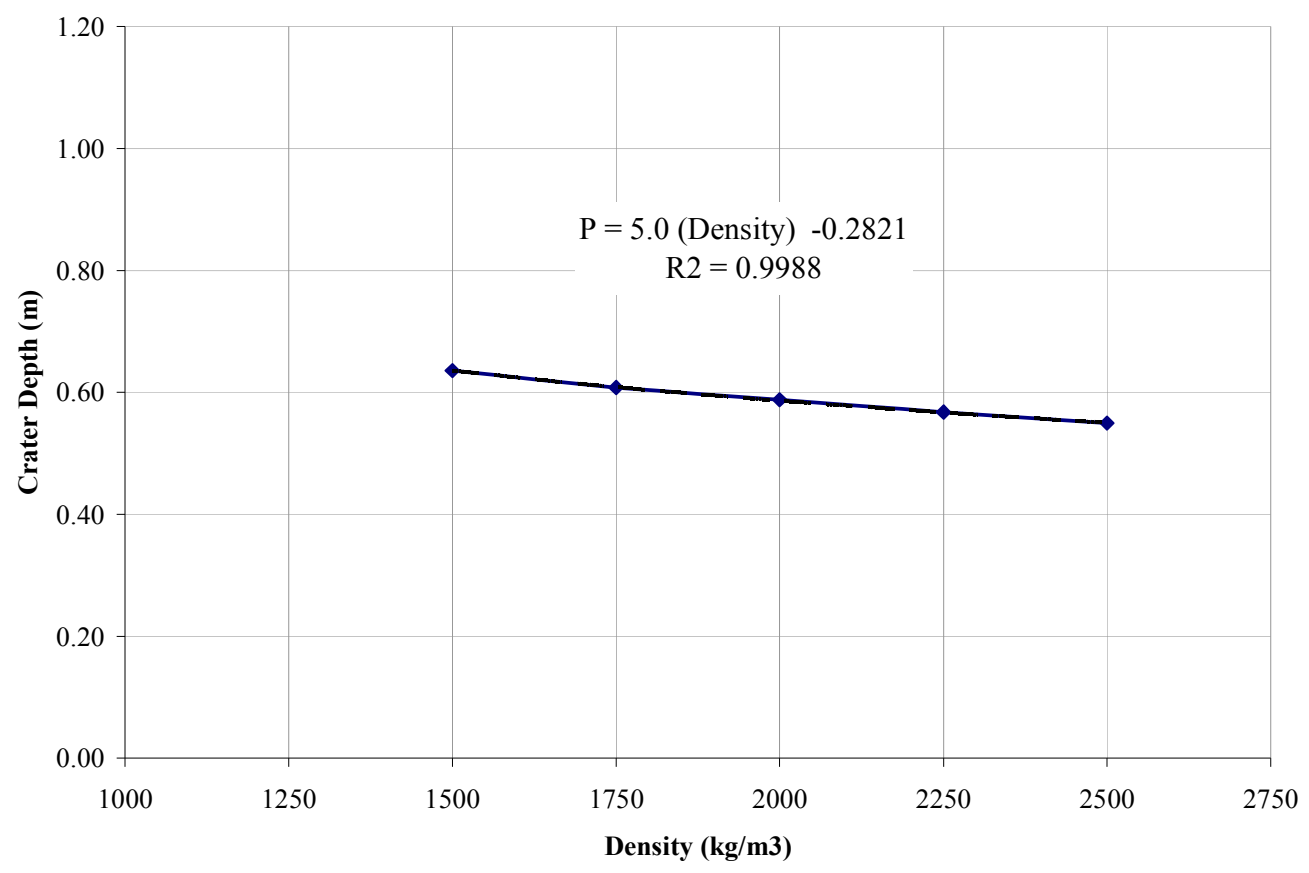

Figure 4-6 Variation of crater depth with initial soil density 


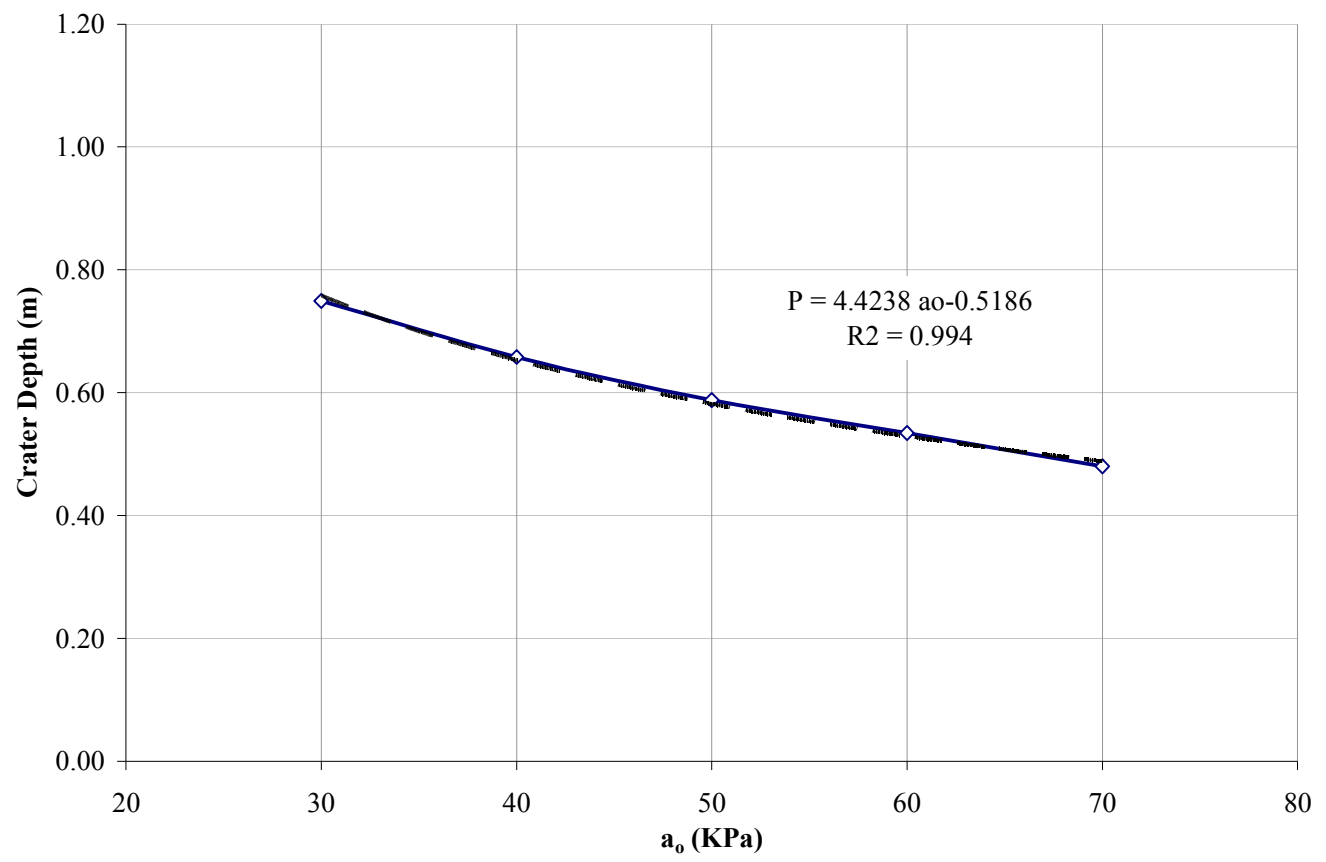

Figure 4-7 Variation of crater depth with pre-consolidation pressure

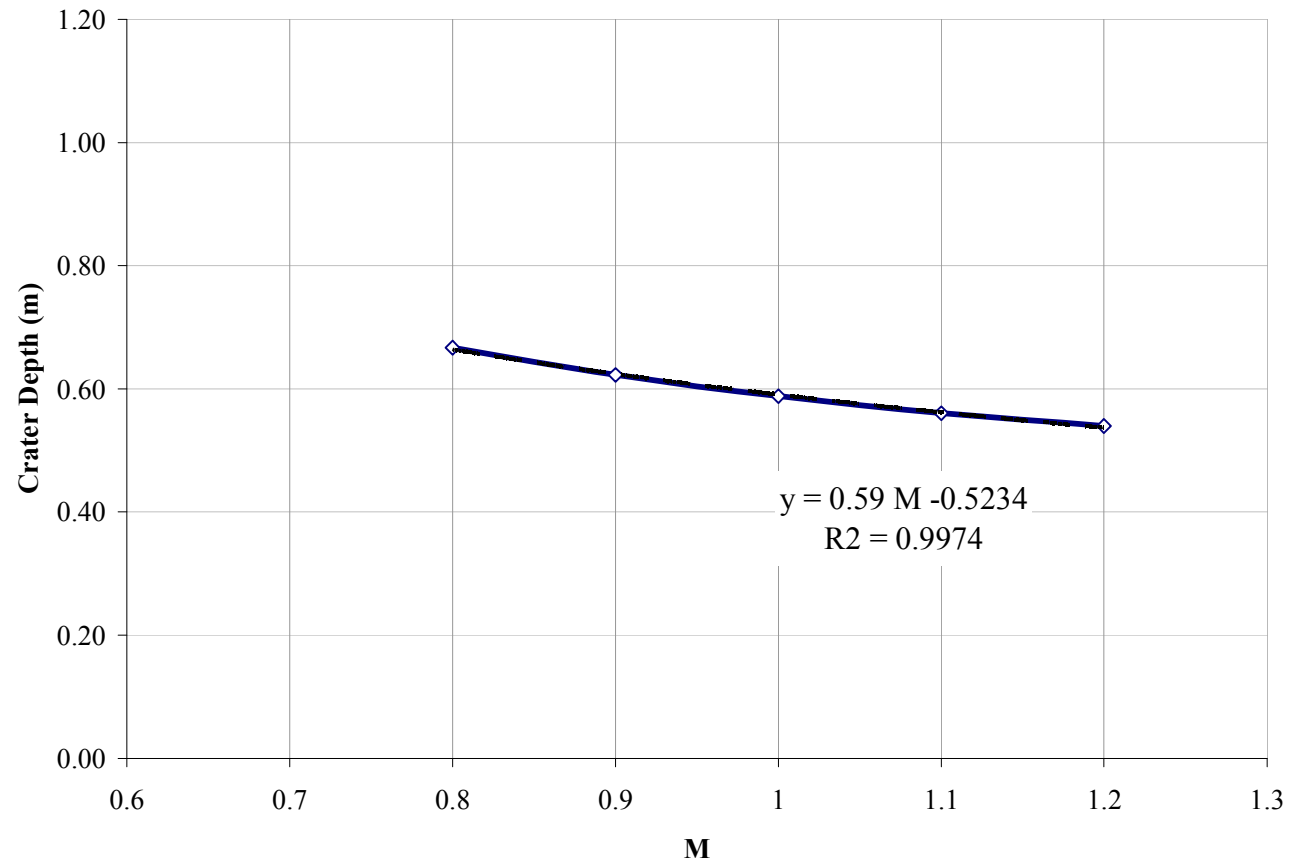

Figure 4-8 Variation of crater depth with CSL slope 


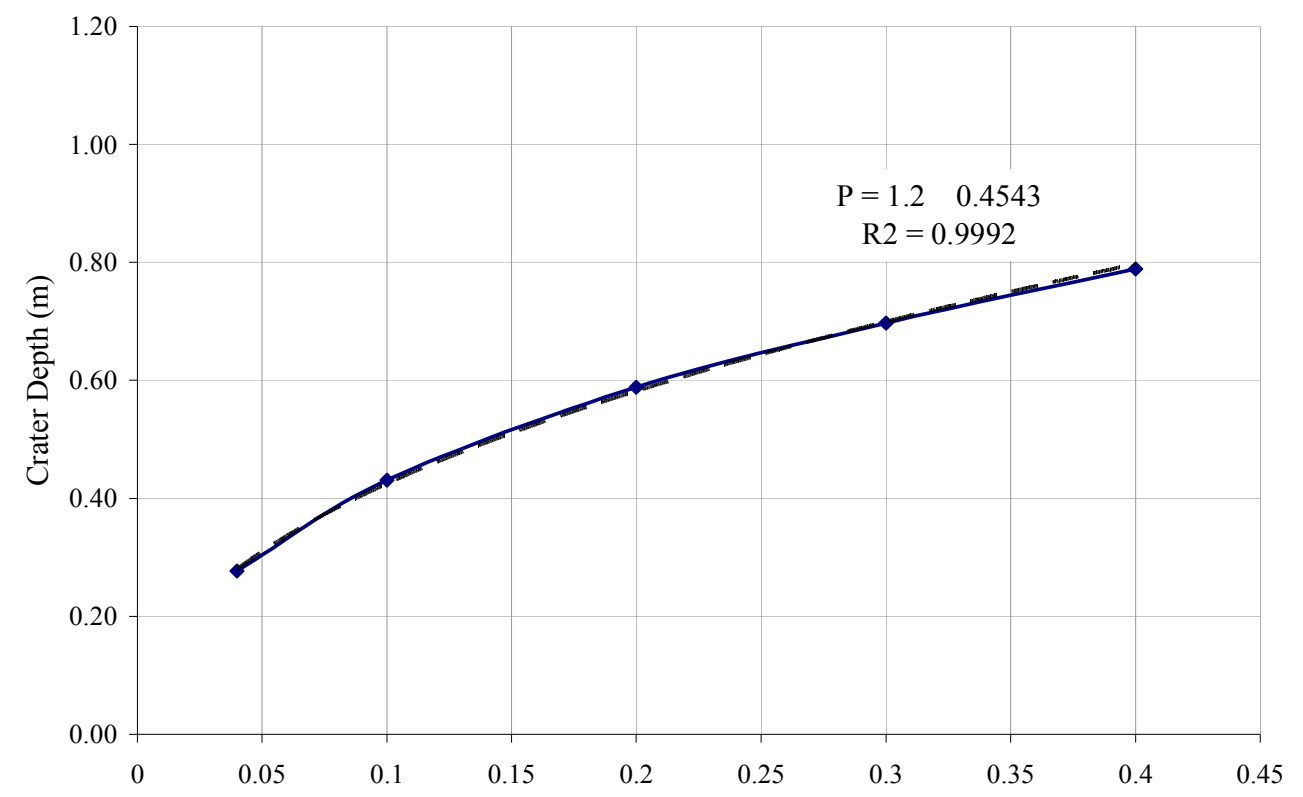

Figure 4-9 Variation of crater depth with logarithmic plastic bulk modulus $(\lambda)$

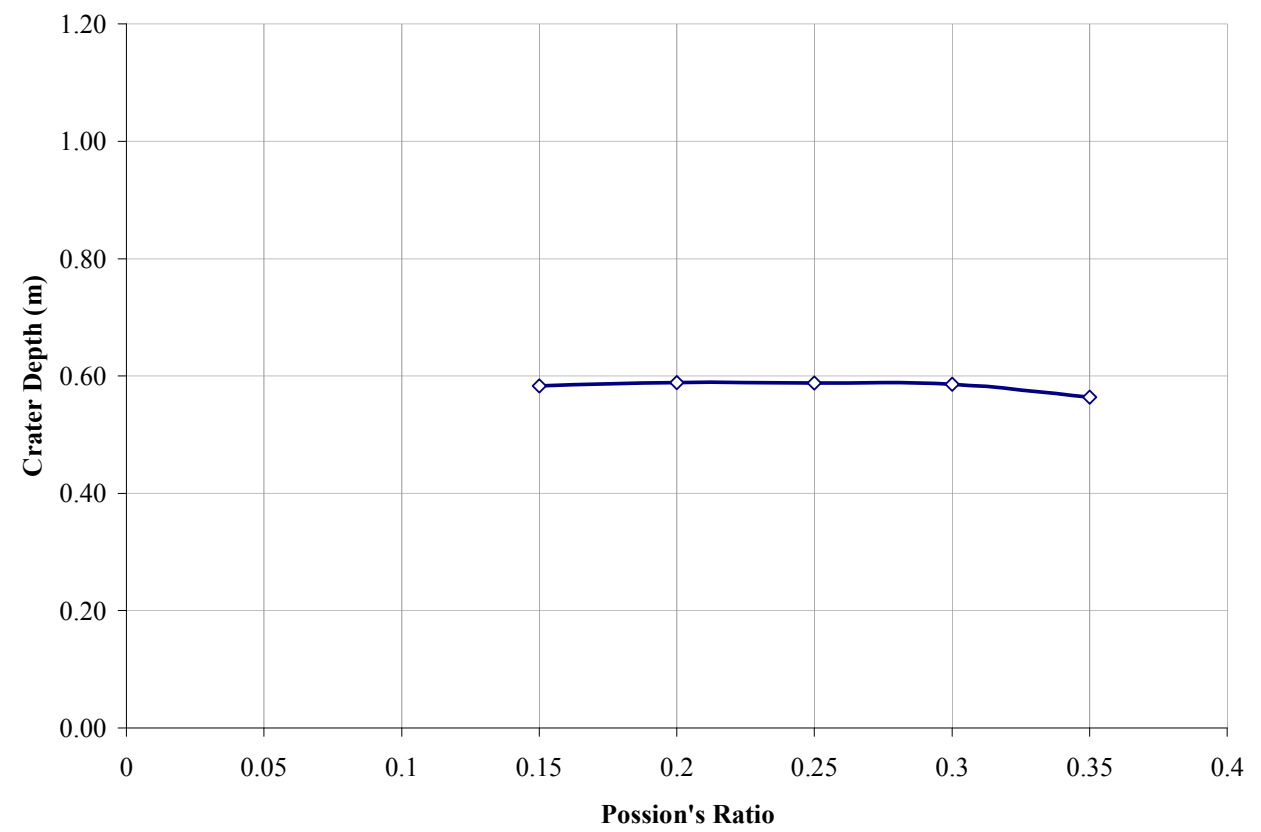

Figure 4-10 Variation of crater depth with Poisson's Ratio 


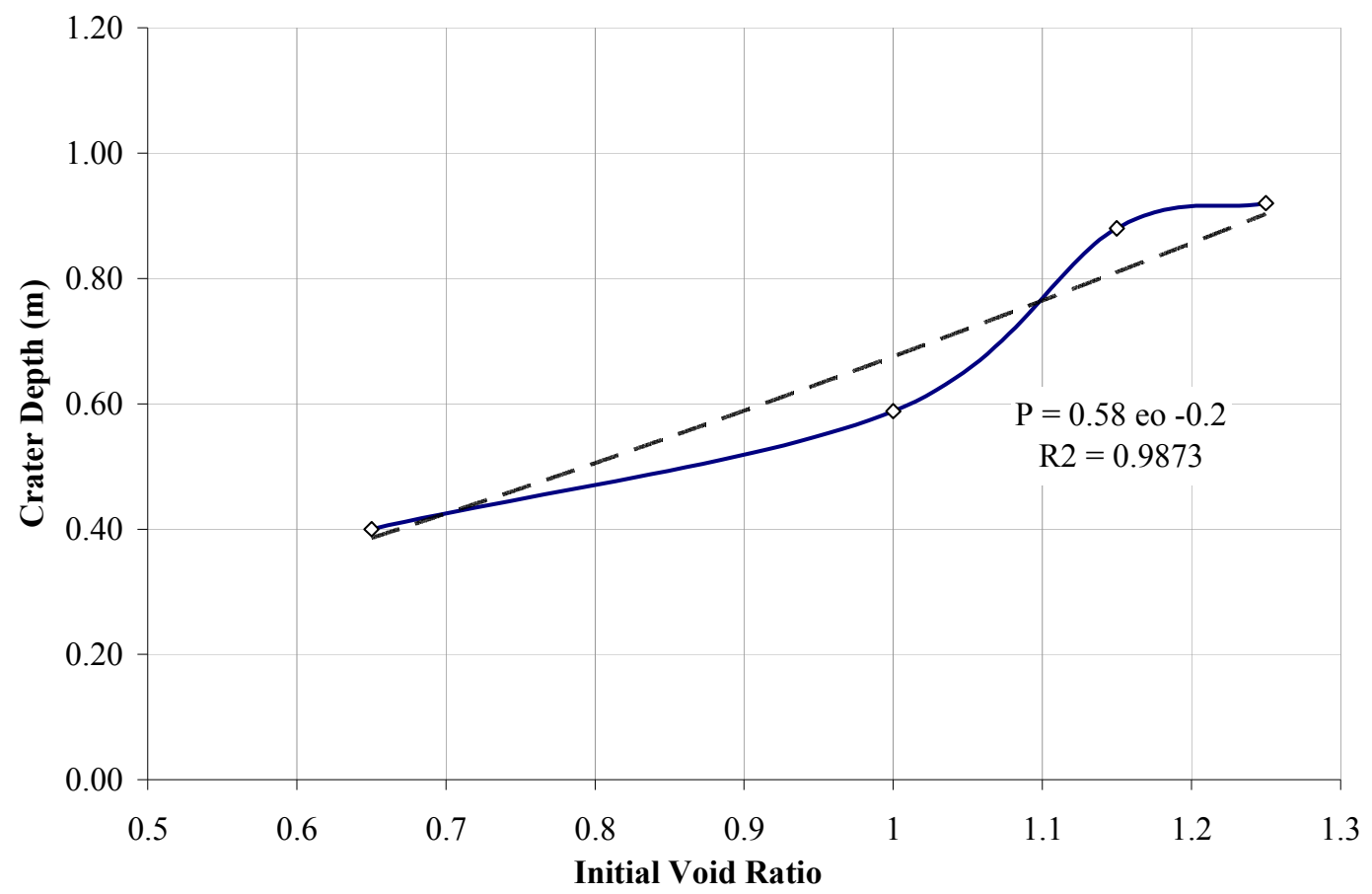

Figure 4-11 Variation of crater depth with initial void ratio

To show the relative importance of soil parameters on the crater depth and the influence of varying soil properties on the crater depth, Figure 4-12 was prepared. The horizontal axis represents the normalized (to the base line value) soil property. The vertical axis represents the normalized crater depth (to the crater depth of the base line model). It can be noticed that the relative variations in soil properties has significant influence on change in crater depth. This may help explain the wide variation of crater depth data collected in the literature. 


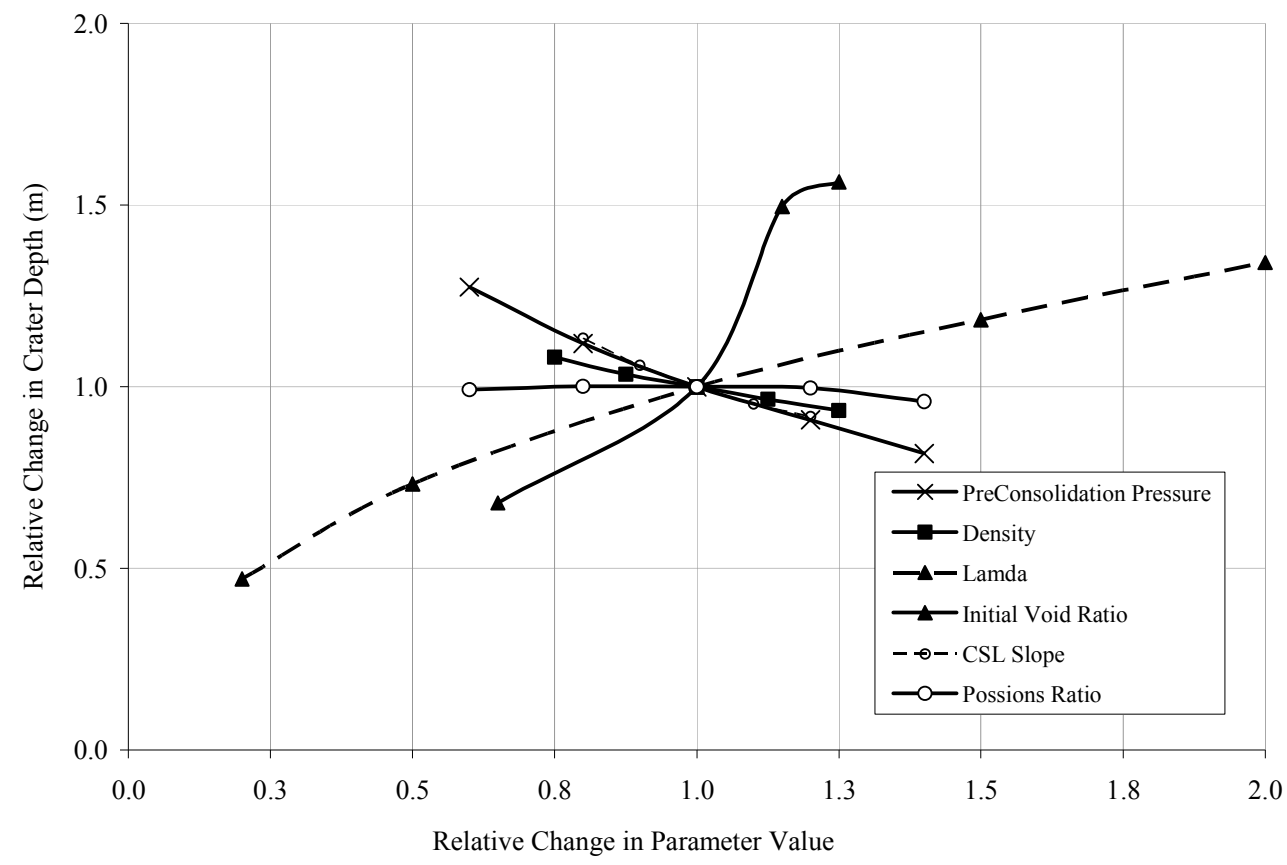

Figure 4-12 Relative change in crater depth vs. relative chance in soil properties

Table 4-3 summarizes the parametric sensitivity analysis results, in which the parameter $\mathrm{C}$ in Equation (4-1) was calculated for each simulation results. The same results presented in Table 4-3 are shown in Figure 4-13 as a bar chart. It can be seen that the order of parameters affecting the crater depth can be ranked from high to low as follows: $\left(e_{o}, \lambda, a_{o}, M, \rho, v\right)$. It can be noticed also that Poisson's ratio has exerted relatively small effects on the crater depth. Therefore, Poisson's ratio was not included through out the subsequent statistical analysis for finding the relationship between $\mathrm{C}$ and Cam-Clay model parameters.

The results of FE simulations are analyzed by using a statistical analysis software SPSS (Statistical Package for the Social Sciences, 2003) program. It was assumed for the purpose of nonlinear regression, that $\mathrm{C}$ could be computed as in Equation (4-2) by a product of various $g$ functions. Each of those functions represents the variation of the 
parameter with one of soil properties. Equation (4-3) represents the correlation obtained from SPSS.

Goodness of fit of the nonlinear regression analysis was examined using Rsquared which was found 0.9512 between the predicted crater depths and the ones obtained from FE simulations as seen in Figure 4-14. The R-squared value is considered to be fairly good. It is also important to mention that, to make the Equation (4-3) for the parameter $\mathrm{C}$ useable, it has been selected to be a multiplication of a series of power functions. Based on the analysis presented, the formula is considered statistically reliable in the range of parameters used for the study.

$$
\begin{aligned}
& C=g_{1}(\lambda) g_{2}\left(e_{o}\right) g_{3}(\rho) g_{4}(M) g_{5}\left(a_{o}\right) \\
& C=\frac{1.8 e_{o}^{1.93} \lambda^{0.04}}{\rho^{0.56} M^{0.3} a_{o}^{0.53}}
\end{aligned}
$$

Table 4-3 Sensitivity analysis of parameter C

\begin{tabular}{l|c|c|c|c|c|c}
\hline & $\rho$ & $v$ & $M$ & $a_{o}$ & $e_{o}$ & $\lambda$ \\
\hline Max & 0.022 & 0.021 & 0.023 & 0.026 & 0.032 & 0.024 \\
\hline Min & 0.019 & 0.020 & 0.019 & 0.017 & 0.014 & 0.015 \\
\hline Max-Min & 0.003 & 0.001 & 0.004 & 0.009 & 0.018 & 0.009 \\
\hline Max-Min/ S(Max- Min) (\%) & $6.7 \%$ & $1.9 \%$ & $9.8 \%$ & $20.8 \%$ & $40.2 \%$ & $20.6 \%$ \\
\hline
\end{tabular}




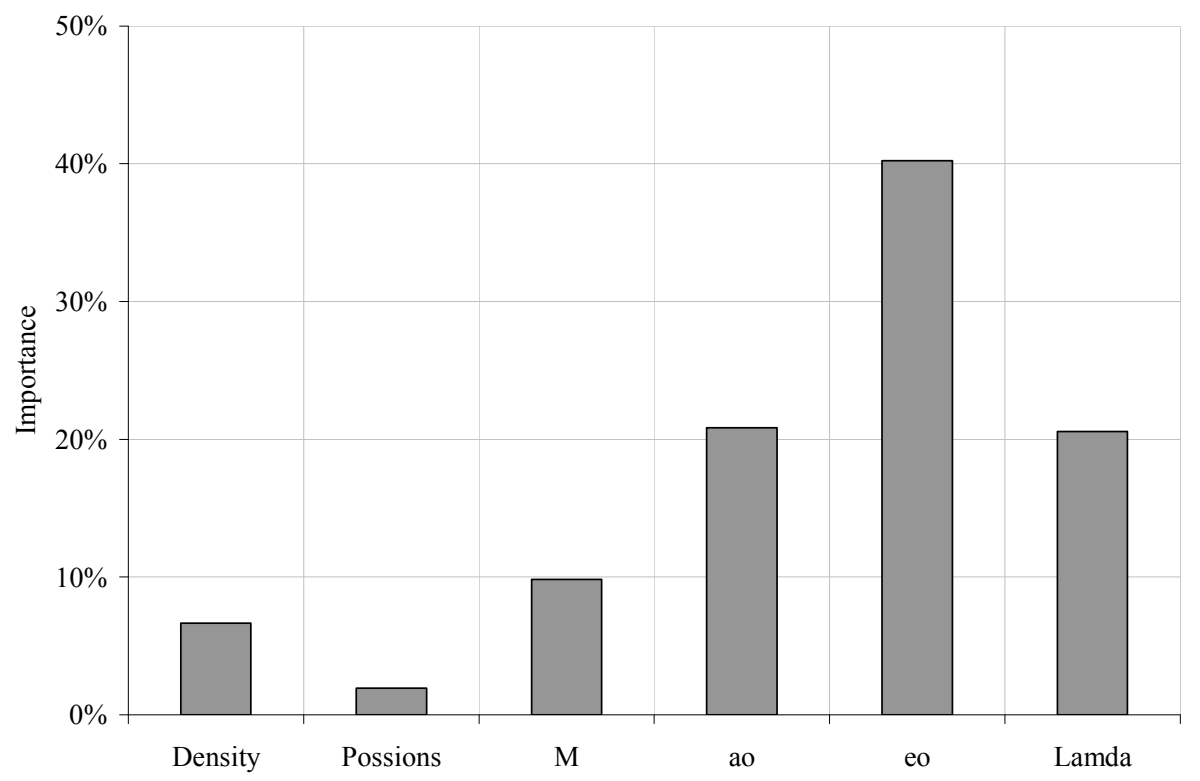

Figure 4-13 Sensitivity of crater depth to soil propeties

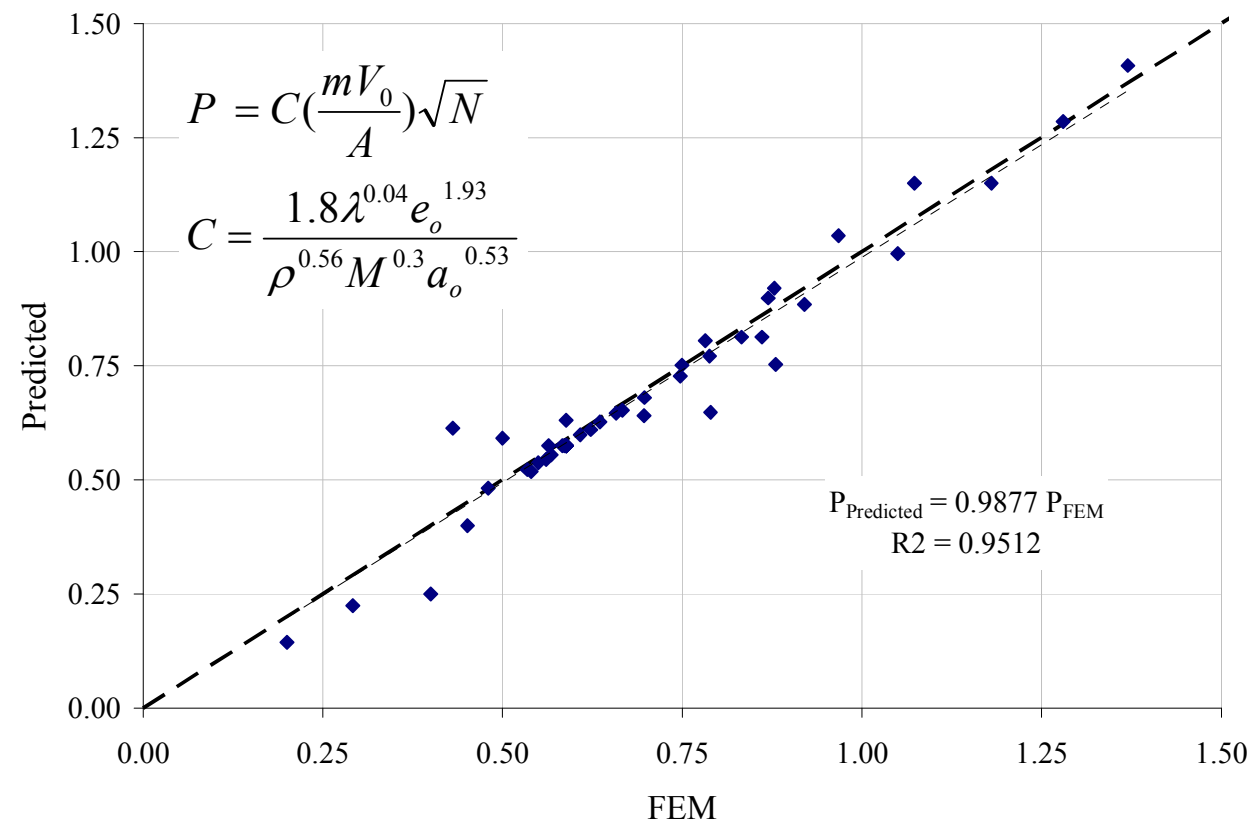

Figure 4-14 Comparison of the predicted crater depth with crater depth obtained from the FEM 


\subsection{Zone of Influence}

As stated earlier, the work done by various researchers had attempted to correlate the maximum depth of influence to the tamping energy as $n \sqrt{W H}$, where $W$ is the tamper mass in tons, $H$ is the drop height is meters, $n$ is a multiplier which varies according to soil type and condition. The depth of maximum improvement is the depth at which the highest degree of improvement is noticed, which usually is located at $1 / 2$ to $1 / 3$ of the maximum depth of improvement measured from the bottom of the crater. Mayne et al. (1984) investigated the degree of improvement and the depth of improvement by comparing the SPT, CPT and PMT before and after compaction for three sites in Virginia.

Utilizing the results from the present parametric study, Figure 4-15 shows a summary of the FE results obtained. The chart represents the cases where soil properties were kept constant at the baseline properties while the tamping energy was varied. Those are the cases with the numerical value of $\sqrt{W H}$ being 12 (ton-meter) and above. For this set of cases, the multiplier $n$ is around 0.4. Cases in which tamping energy was kept constant while the soil properties were changed are plotted on Figure 4-15 as well. Those cases are represented by a group of vertically aligned points at $\sqrt{W H}$ equal to 10 (tonmeter). The maximum depth of influence in meter, which is measured from the bottom of crater and along the line of symmetry, varies from 0.35 to $0.6 \sqrt{W H}$. It can be concluded that variations in soil properties can exert significant effects on the depth of influence. In addition, the current models of DC in predicting depth of influence are overly simplified as they generally do not consider the effects of the variation of soil properties before 
compaction. Furthermore, the current prediction models can not predict soil properties after compaction. Conceptually, the depth of maximum improvement is the depth at which the maximum relative density is achieved. Knowing that the void ratio distribution can be obtained as FE simulation output, and since Cam-clay constitutive model was used for the analysis, the influence of tamping energy and soil properties on void ratio distribution is investigated in the this section.

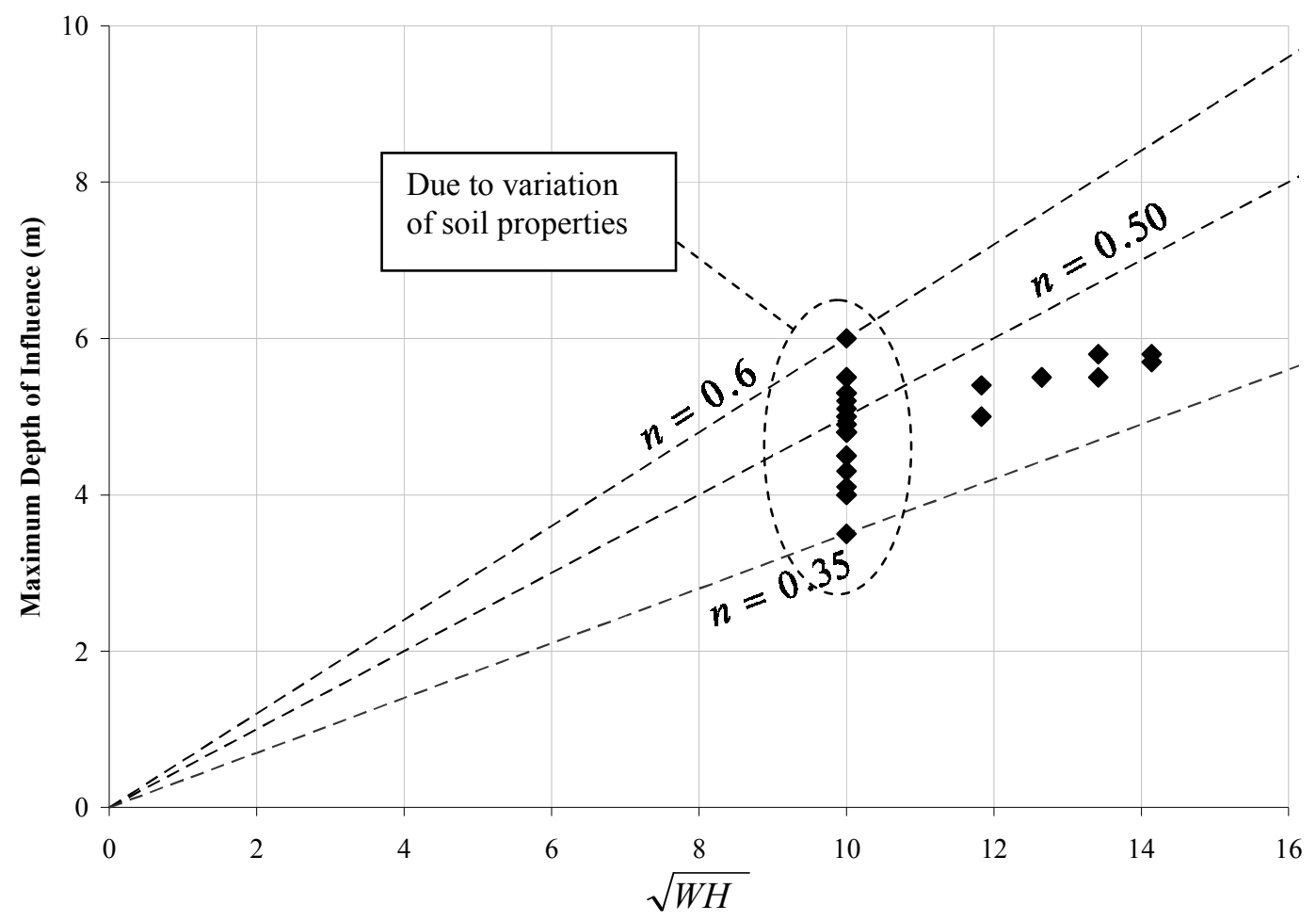

Figure 4-15 Summary of depth of influence

Figure 4-16 shows the development of zone of influence with six consecutive drops. The influence zone is divided into three areas based on void ratio distribution (blow 0.65 , below 0.75 and below 1.00). The mechanism of compaction can be understood and summarized as follows 
- Zone 1 , which is the maximum zone of improvement, is formed directly beneath the tamper after the first drop. The zone has a rectangular shape.

- Zone 1 increases in the vertical direction almost without increase in the horizontal (lateral) direction with the subsequent tamper drops.

- Zone 2 is a zone with average and moderate improvement and has circular shape.

- Zone 2 increases rapidly in the horizontal and vertical directions with the consecutive tamper drops.

- Zone 3 is a zone with the least improvement and has the semi- spheroid shape.

- Zone 3 increases both horizontally and vertically, however, with most of the increase occurs in the horizontal direction.

It can be concluded that the degree of compaction varies in the vertical and horizontal directions. The current approach in using a single multiplier to estimate the dimensions of the maximum zone of influence appears to be overly simplified due to a lack of consideration of the influence of the other important influencing parameters.

The first step in the analysis is to investigate the influence of tamping energy on the void ratio distribution. Figure 4-17 to Figure 4-19 show the void ratio distribution along the depth (measured from the bottom of the crater) for different cases in which tamper mass, tamping velocity and tamper cross sectional area were changed while keeping soil properties constant. Figure 4-17 shows the void ratio distribution along the depth for varying tamper mass while keeping soil properties and drop height and tamper radius constant. Figure 4-18 shows the variation of void ratio distribution along the depth 
for varying drop height while keeping the tamper mass and radius as well as soil properties constant. Figure 4-19 shows the variation of void ratio distribution along the depth for varying tamper radius while keeping the drop height and the tamper mass constant. Reducing the tamper radius results in high energy density per unit area, hence, higher reduction in void ratio occurs. Significant influence of tamper characteristics can be noticed on the void ratio distribution.

Figure 4-20 was developed to investigate the variation of $\Delta e_{o}$, reduction in void ratio, with the scaled energy $\sqrt{W H} / A$, which is the normalized energy per blow. The reduction in void ratio is plotted at different depths at 1 meter, 2 meters, and 3 meters, respectively. The goodness of linear regression is examined using R-squared. R-squared for the three depths were found to be $0.9237,0.9696$, and 0.9345 for the three depths listed above, respectively. A good fit could be achieved for the linear model. Therefore, the reduction in void ratio at any depth is linearly proportional to the scaled energy $\sqrt{W H} / A$.

The same analysis approach was conducted to investigate the influence of number of drops on the reduction in void ratio. The reduction in void ratio is plotted for 1 meter, 2 meters and 3 meters depths as shown in Figure 4-21. The reduction in void ratio is approximately proportional to the square root of number of drops. R-squared for the three depths were found to be $0.9519,0.9827$, and 0.9814 , respectively. The above noted observations can be summarized mathematically as follows:

$$
\begin{aligned}
& \text { - } \Delta e_{0} \alpha \frac{\sqrt{W H}}{A} \\
& \text { - } \Delta e_{0} \alpha \sqrt{N}
\end{aligned}
$$


- $\Delta e_{0}$ exponentially decays with depth

Based on the correlations presented in Figure 4-16 to Figure 4-21 and the accompanying discussion presented, the following correlation model was proposed to estimate the reduction of void ratio as a function of scaled tamping energy, depth, and number of drops.

$\Delta e_{0}=C_{1} e^{-C_{2} y}\left(\frac{\sqrt{W H}}{A}\right) \sqrt{N}$

where:

$C_{1}$ and $C_{2}$ : regression parameters which depend on soil properties.

$y$ : Depth measured along the tamper line of symmetry measured from the carter bottom. 


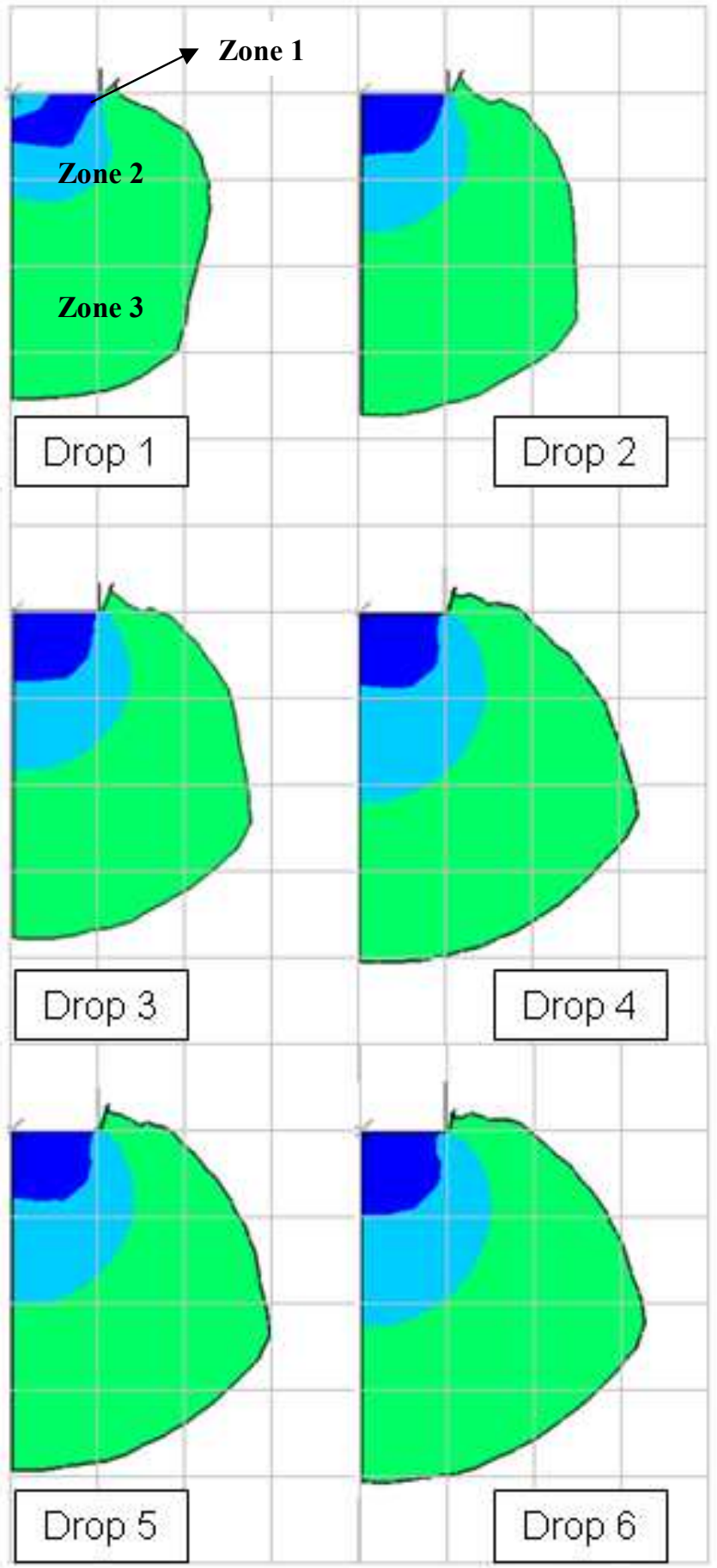

Figure 4-16 Development of zone of influence with multiple drops

(Each square grid has same dimensions as radius of tamper) 


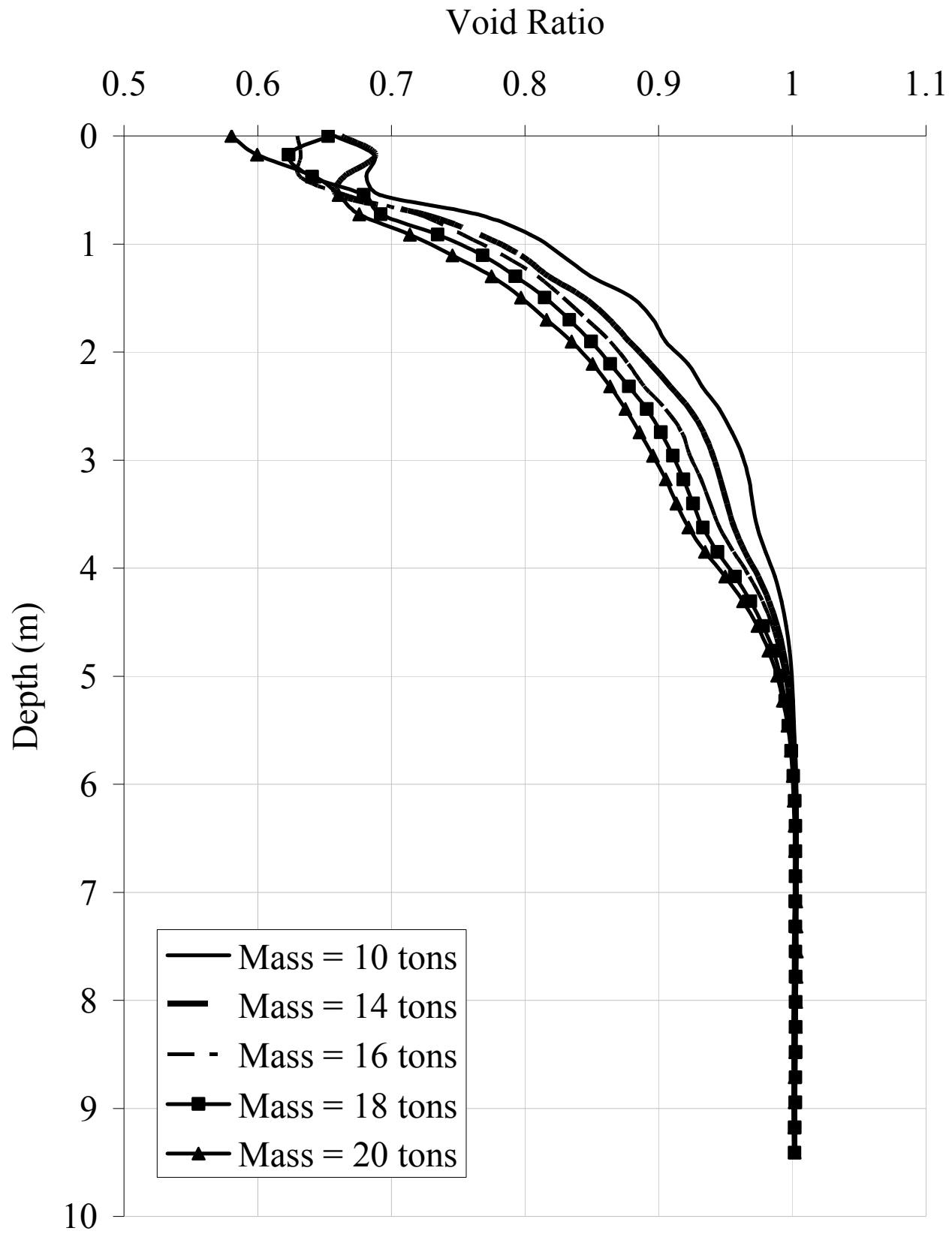

Figure 4-17 Variation of void ratio distribution with tamper mass 


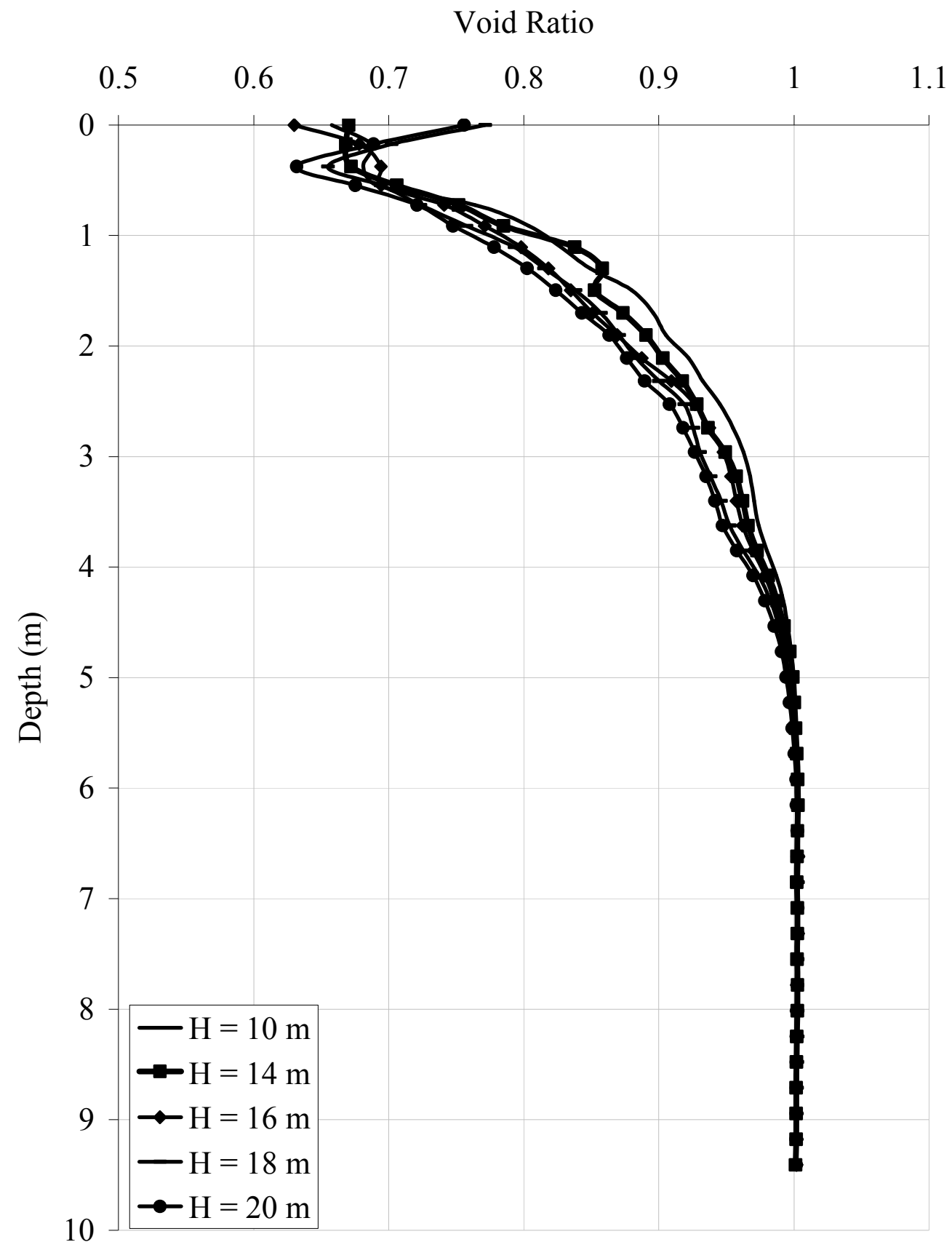

Figure 4-18 Variation of void ratio distribution with drop height 


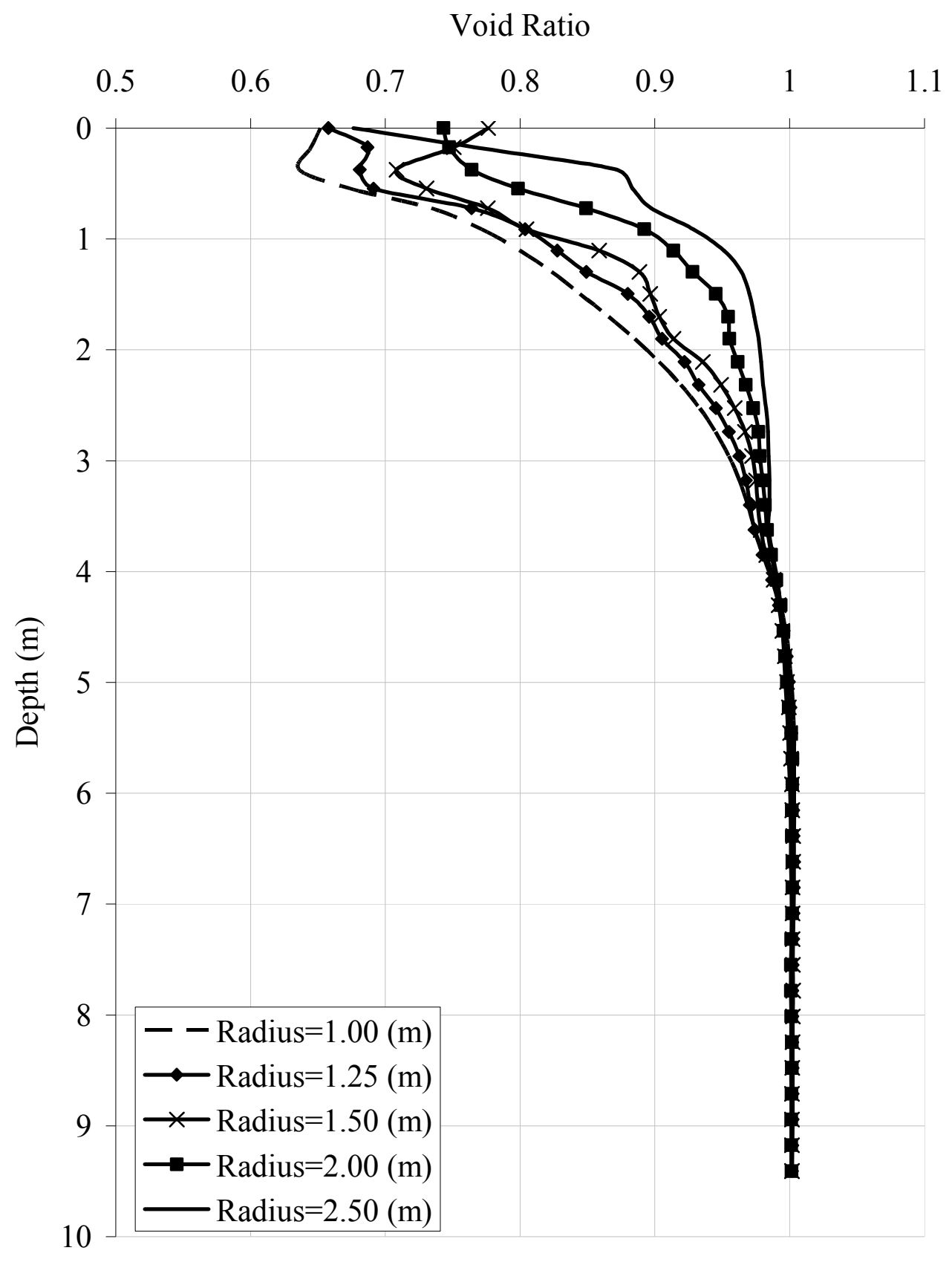

Figure 4-19 Variation of void ratio distribution with tamper radius 


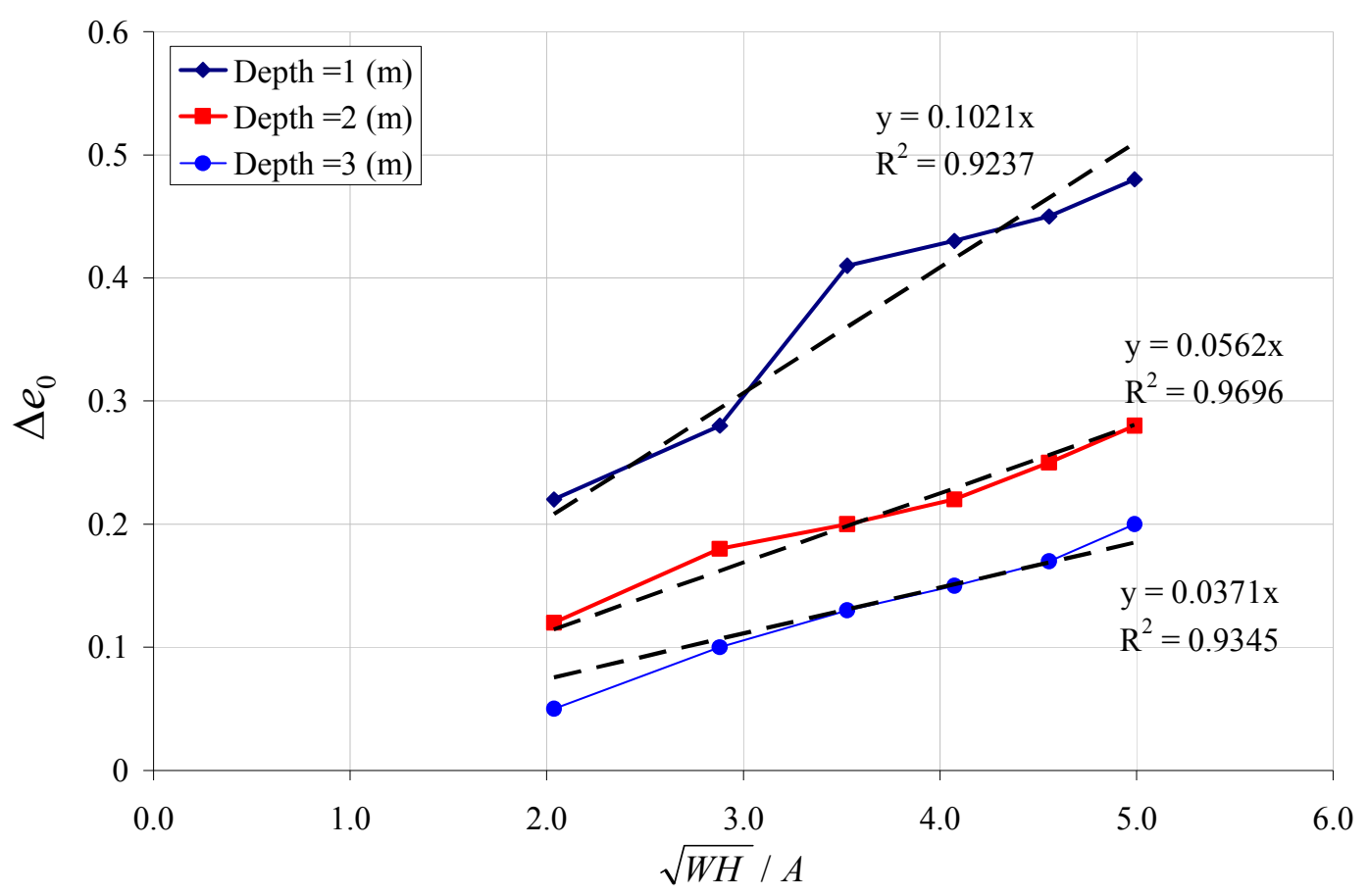

Figure 4-20 Void ratio reduction variations with normalized energy per blow

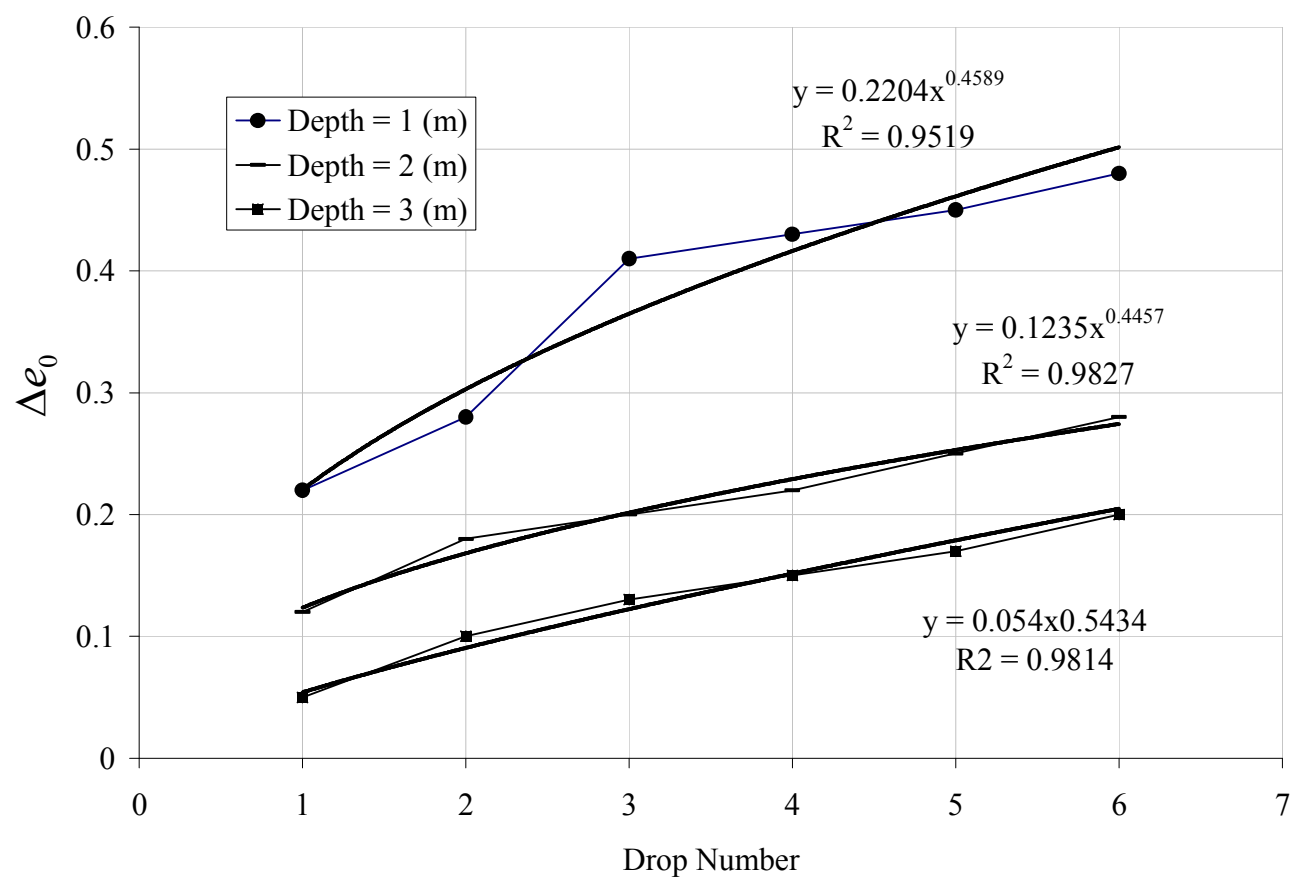

Figure 4-21 Void ratio reduction variations with number of drops 
The second step in the analysis investigates the influence of soil properties on soil void ratio distribution for a given prescribed tamper impact parameters. Figure 4-22 shows the variation of void ratio distribution along the depth for varying initial soil density. Figure 4-23 shows the variation of void ratio distribution along the depth with pre-consolidation pressure. The degree of improvement decreases with increasing preconsolidation pressure. Figure 4-24 shows the variation of void ratio with Critical State Line (CSL) slope (M). Figure 4-25 shows the variation of void ratio with logarithmic plastic bulk modulus $(\lambda)$. It can be noticed that $\lambda$ has a huge influence on the void ratio distribution. Poisson's ratio $(v)$ has slight influence on the void ratio distribution as can be seen in Figure 4-26. Finally, Figure 4-27 shows the variation of void ratio with initial void ratio $\left(e_{o}\right)$.

Although Figure 4-22 to Figure 4-27 give a lot of information about the variation of void ratio along the depth with each soil property, they can not be used directly to derive correlation to estimate the $\mathrm{C}_{1}$ and $\mathrm{C}_{2}$ as function of soil properties. Therefore, using MATLAB curve fitting tool, $C_{1}$ and $C_{2}$ presented in Equation (4-4) were calculated for each case in which soil properties were changed one at a time. The variations of $\mathrm{C}_{1}$ and $\mathrm{C}_{2}$ are shown in Figure 4-28 to Figure 4-33. The proposed correlation is calculated on each plot using MS Excel. The examined R-square value is calculated for each fitting to check for good of fitness.

The physical interpretation of parameters $C_{1}$ and $C_{2}$ in Equation (4-4) is explained herein. The parameter $C_{1}$ indicates the maximum treatment at the surface. This can be obtained by evaluating Equation (4-4) at the soil surface where $y=0$. By substitution, the 
maximum reduction at the surface is equal to $C_{1}$ multiplied by the normalized tamping energy and the square root of number of drops $\left(\Delta e_{0, y=0}=C_{1}\left(\frac{\sqrt{W H}}{A}\right) \sqrt{N}\right)$. Simply, the higher the value of $\mathrm{C}_{1}$ is the higher achievable reduction in void ratio for the same tamping energy per blow and number of drops. $\mathrm{C}_{2}$, on the hand, indicates the maximum decay rate for the void ratio profile along the depth. High $\mathrm{C}_{2}$ value results in fast decay for the function; hence, shallow treatment is expected.

Figure 4-28 shows the variation of $\mathrm{C}_{1}$ and $\mathrm{C}_{2}$ with initial void ratio. By increasing the initial void ratio, $\mathrm{C}_{1}$ increases and $\mathrm{C}_{2}$ decreases. This can be interpreted as when the soil has relatively high initial void ratio, most of the treatment occurs at the surface. The depth of influence is expected to be lower in this case. Two correlations are proposed for using power function. The goodness of fit was examined using R-squared value which was found to be equal to 0.8178 and 0.6175 for $\mathrm{C}_{1}$ and $\mathrm{C}_{2}$, respectively.

Figure 4-29 shows the variation of $\mathrm{C}_{1}$ and $\mathrm{C}_{2}$ with $\mathrm{M}$ which is the slope of critical State Line (CSL). $\mathrm{C}_{1}$ slightly increases by increasing M. Similarly, two correlations are proposed for using power function. The goodness of fit was examined using R-squared value which was found to be equal to 0.8642 and 0.8503 for $C_{1}$ and $C_{2}$ respectively. Figure 4-30 shows the variation of $\mathrm{C}_{1}$ and $\mathrm{C}_{2}$ with initial soil density. No noticeable effect for soil density was noticed on $\mathrm{C}_{1}$ and $\mathrm{C}_{2}$.

Variation of $C_{1}$ and $C_{2}$ with Poisson's ratio is shown in Figure 4-31. Increasing Poisson's ratio results in decrease in $\mathrm{C}_{1}$. A power function correlation was proposed to estimate $\mathrm{C}_{1}$ a function of Poisson's ratio and the examined using $\mathrm{R}$-squared value (0.7408). $\mathrm{C}_{2}$ is not sensitive to the variation of Poisson's ratio. 
Figure 4-32 shows the variation of $C_{1}$ and $C_{2}$ with logarithmic plasticity bulk modulus $(\lambda)$. It can be noticed that the logarithmic bulk modulus has a significant influence on the values of $\mathrm{C}_{1}$ and $\mathrm{C}_{2}$. The higher value of logarithmic plasticity bulk modulus results in higher reduction in void ratio for the same applied mean effective stress. Similarly, two power functions were proposed for correlation. Examining the goodness of fit showed that good correlation could be achieved. The R-squared values were found to be equal to 0.9883 and 0.9964 for $C_{1}$ and $C_{2}$ respectively. Finally, the influence of increasing the pre-consolidation pressure on $\mathrm{C}_{1}$ and $\mathrm{C}_{2}$ is shown in Figure 4-33. Increasing the pre-consolidation pressure has a significant influence on the value of $\mathrm{C}_{2}$ which results in relatively shallow treatment for the same tamping energy. 


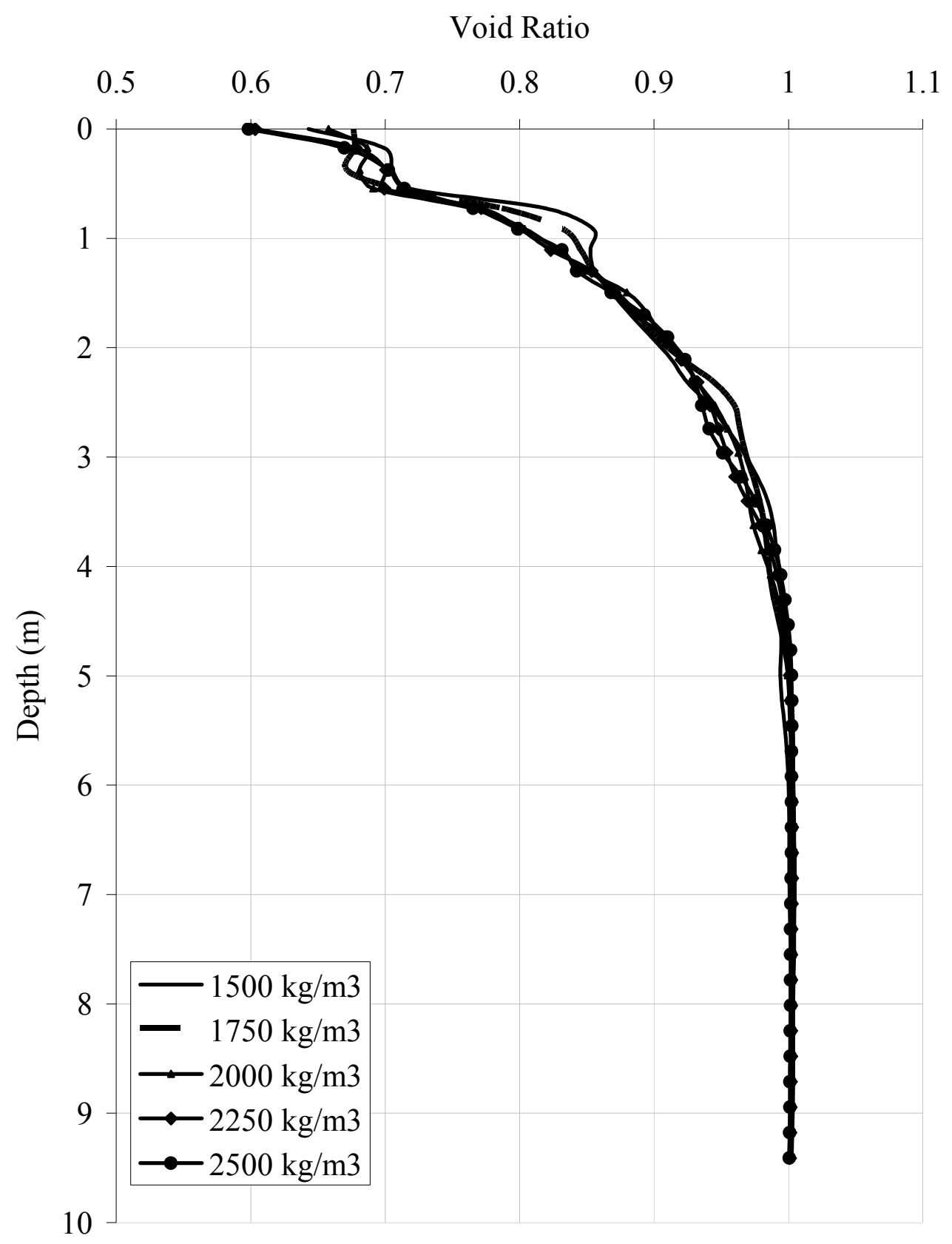

Figure 4-22 Variation of void ratio distribution with initial soil density 


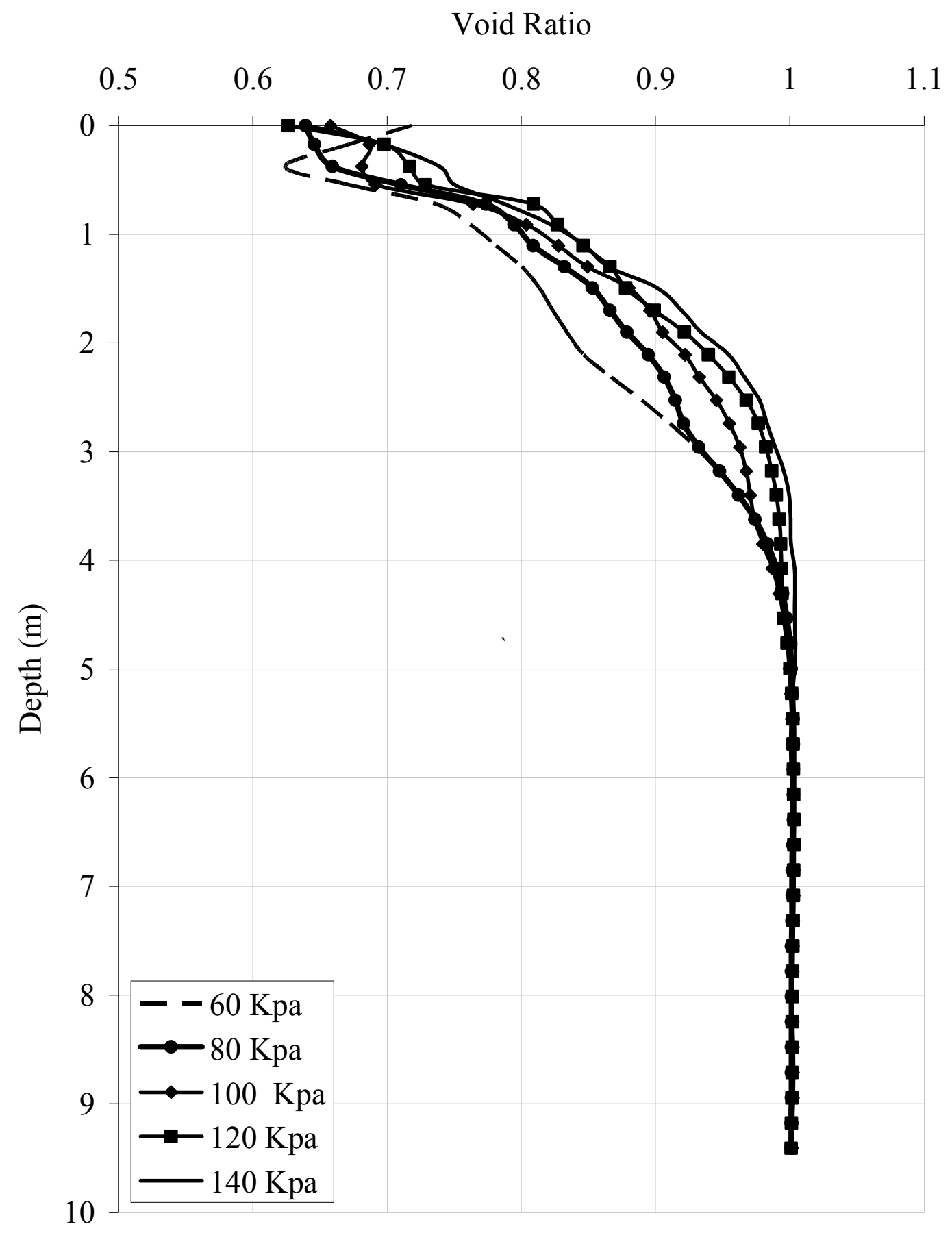

Figure 4-23 Variation of void ratio distribution with pre-consolidation pressure 


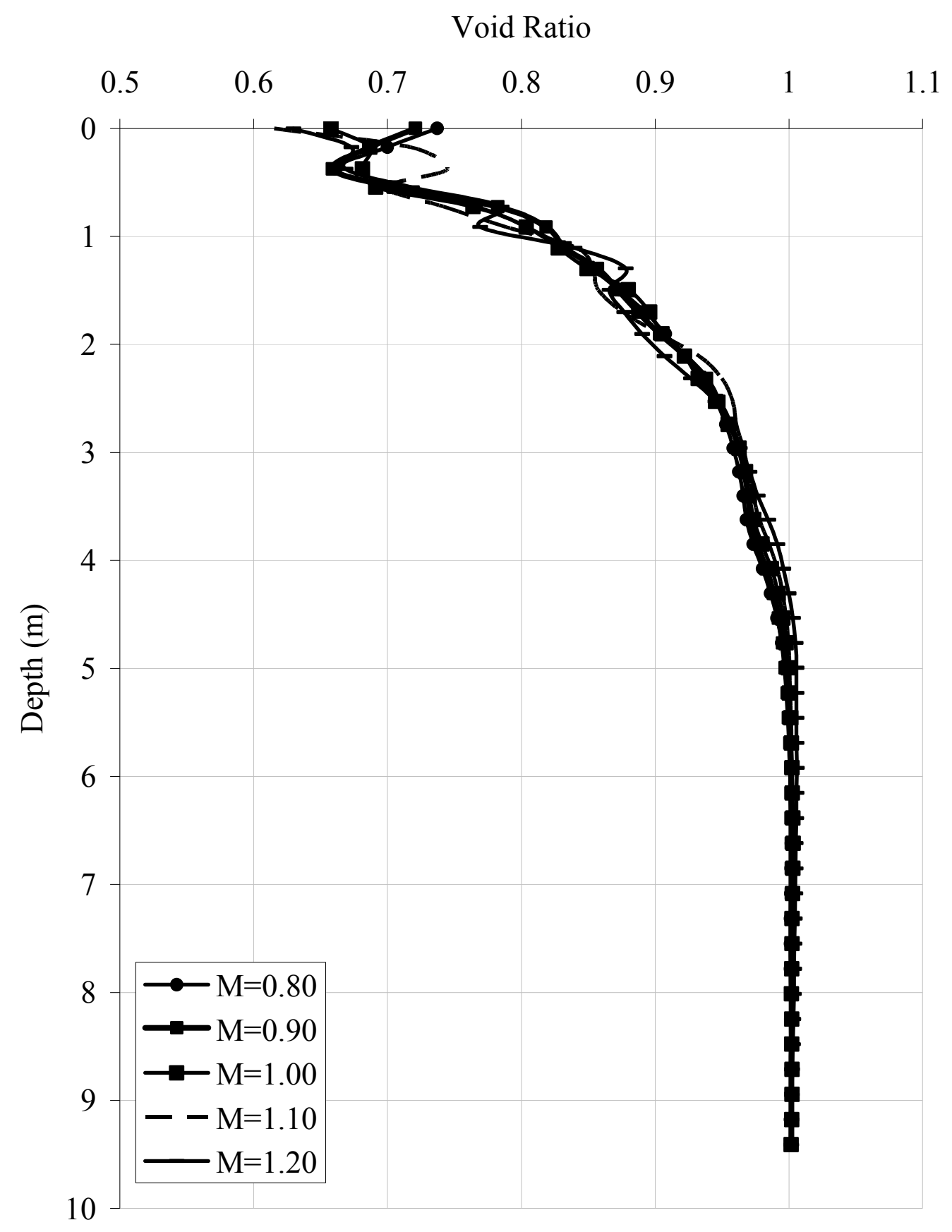

Figure 4-24 Variation of void ratio distribution with CSL slope (M) 


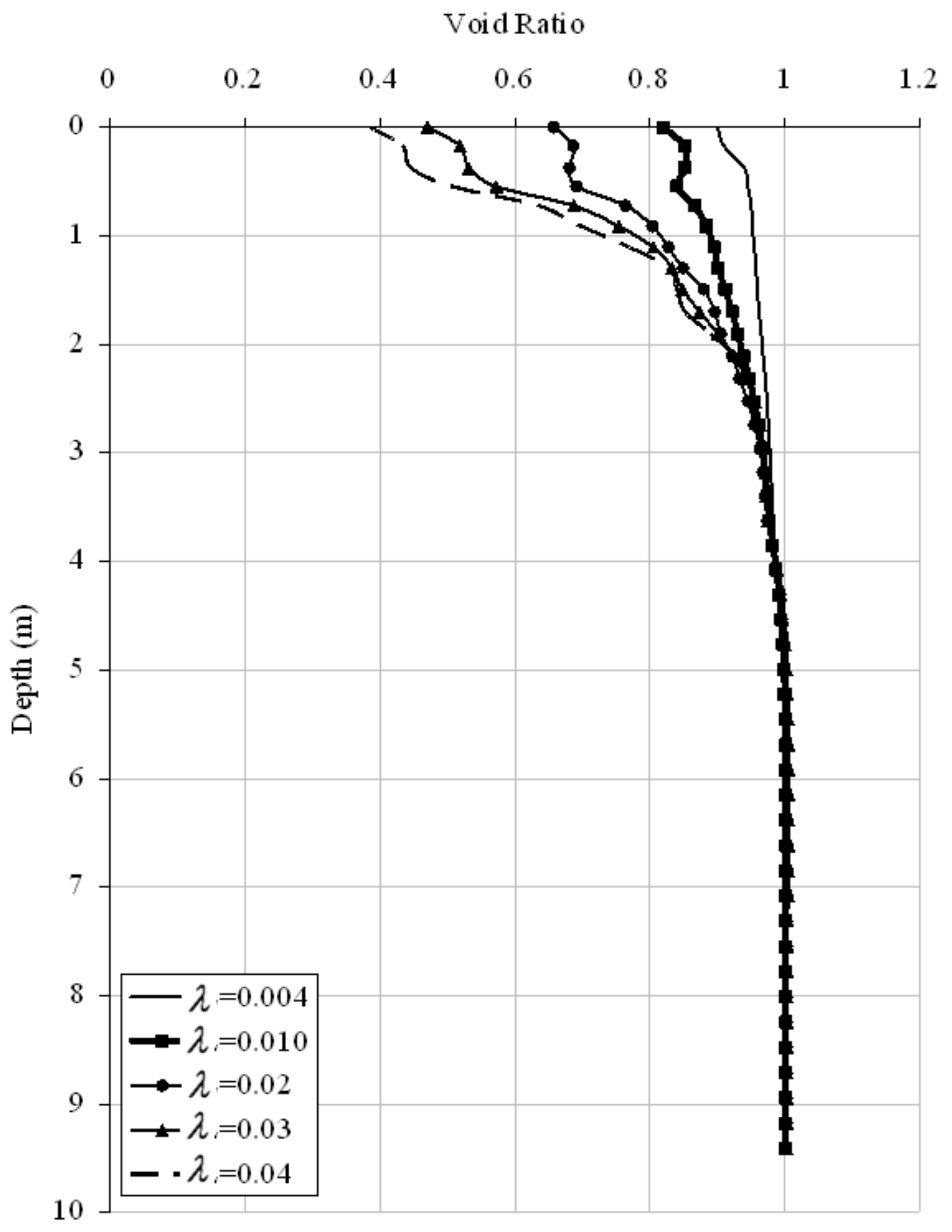

Figure 4-25 Variation of void ratio distribution with $\lambda$ 


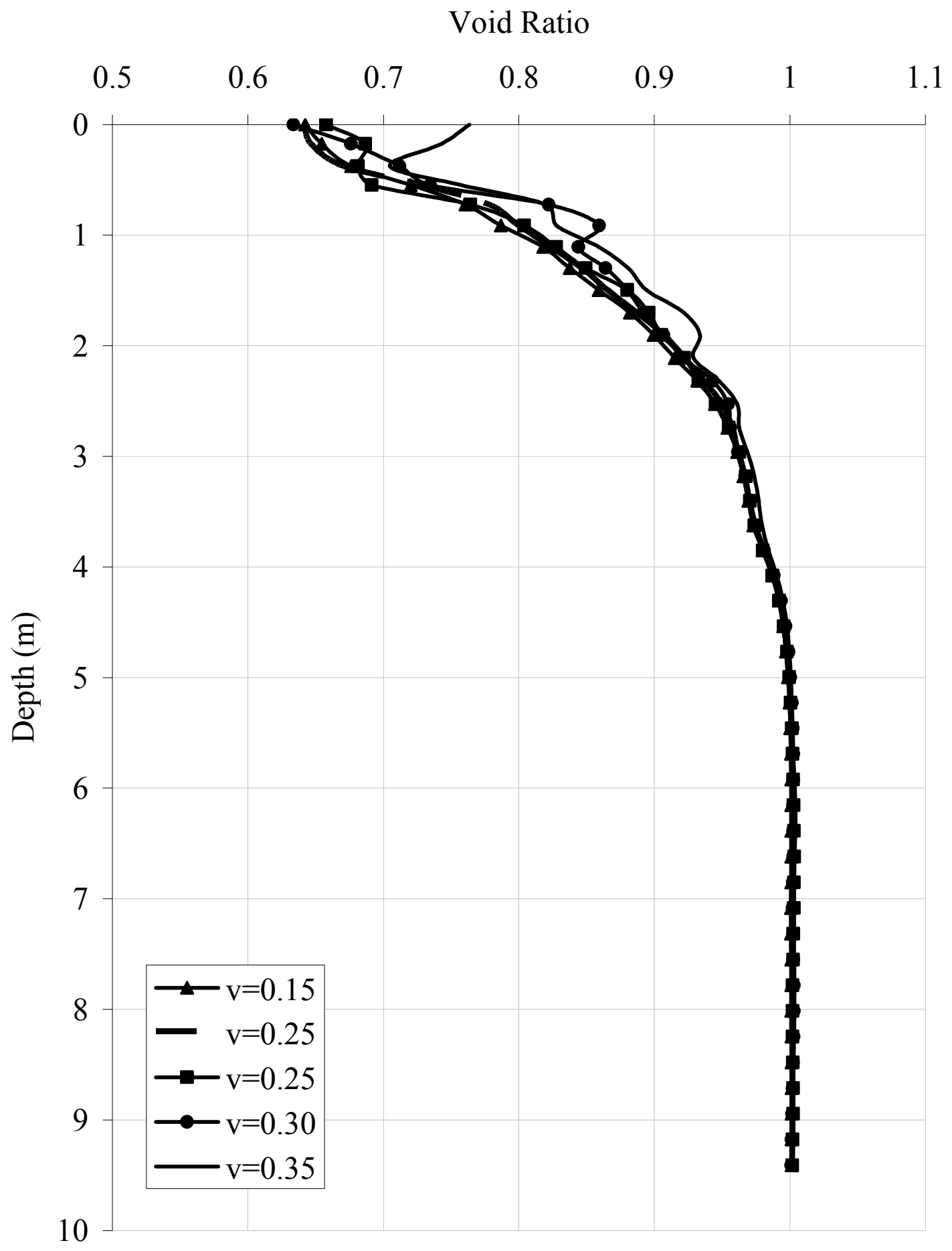

Figure 4-26 Variation of void ratio distribution with Poisson's ratio 


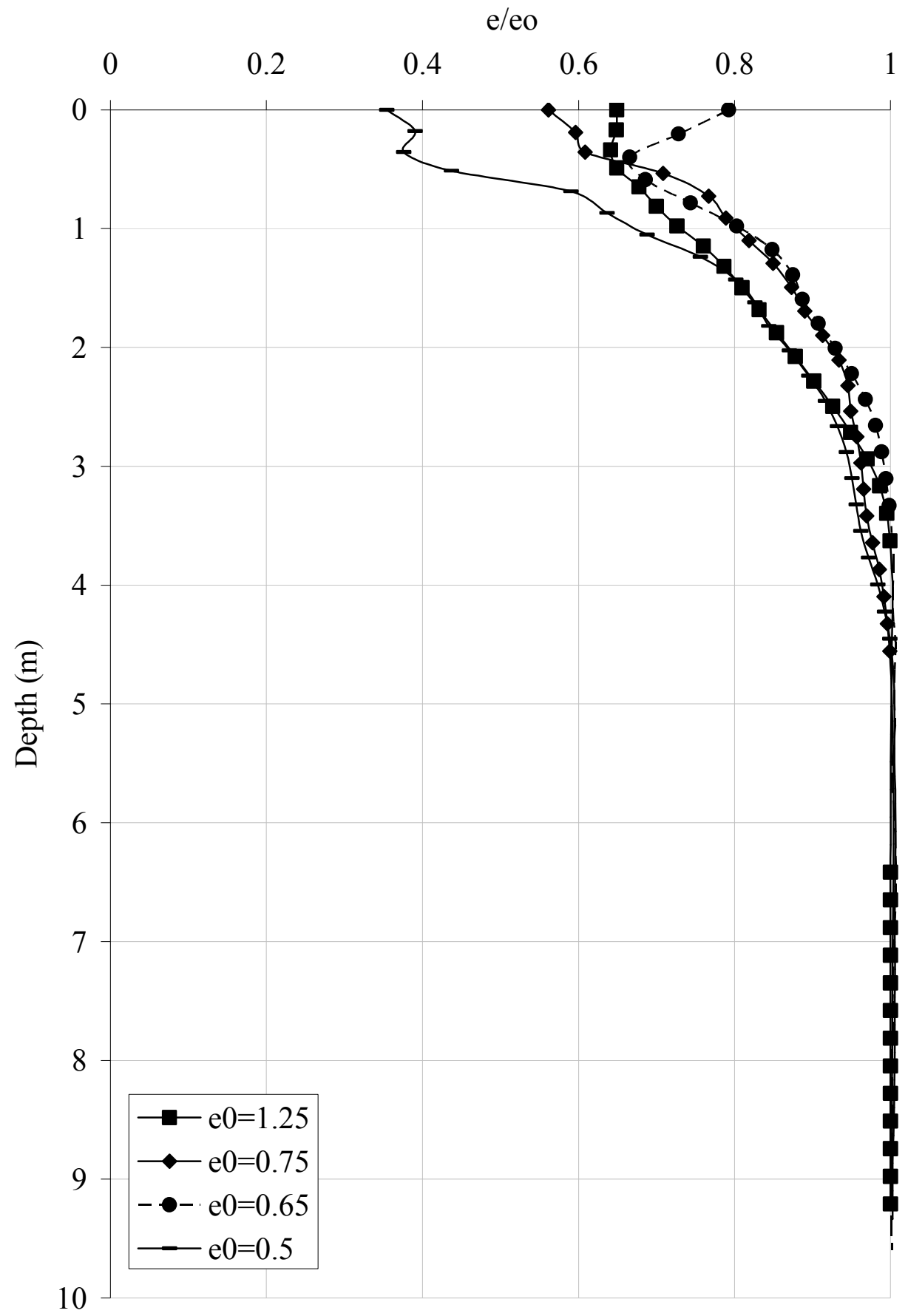

Figure 4-27 Variation of void ratio distribution with the relative change of void ratio 


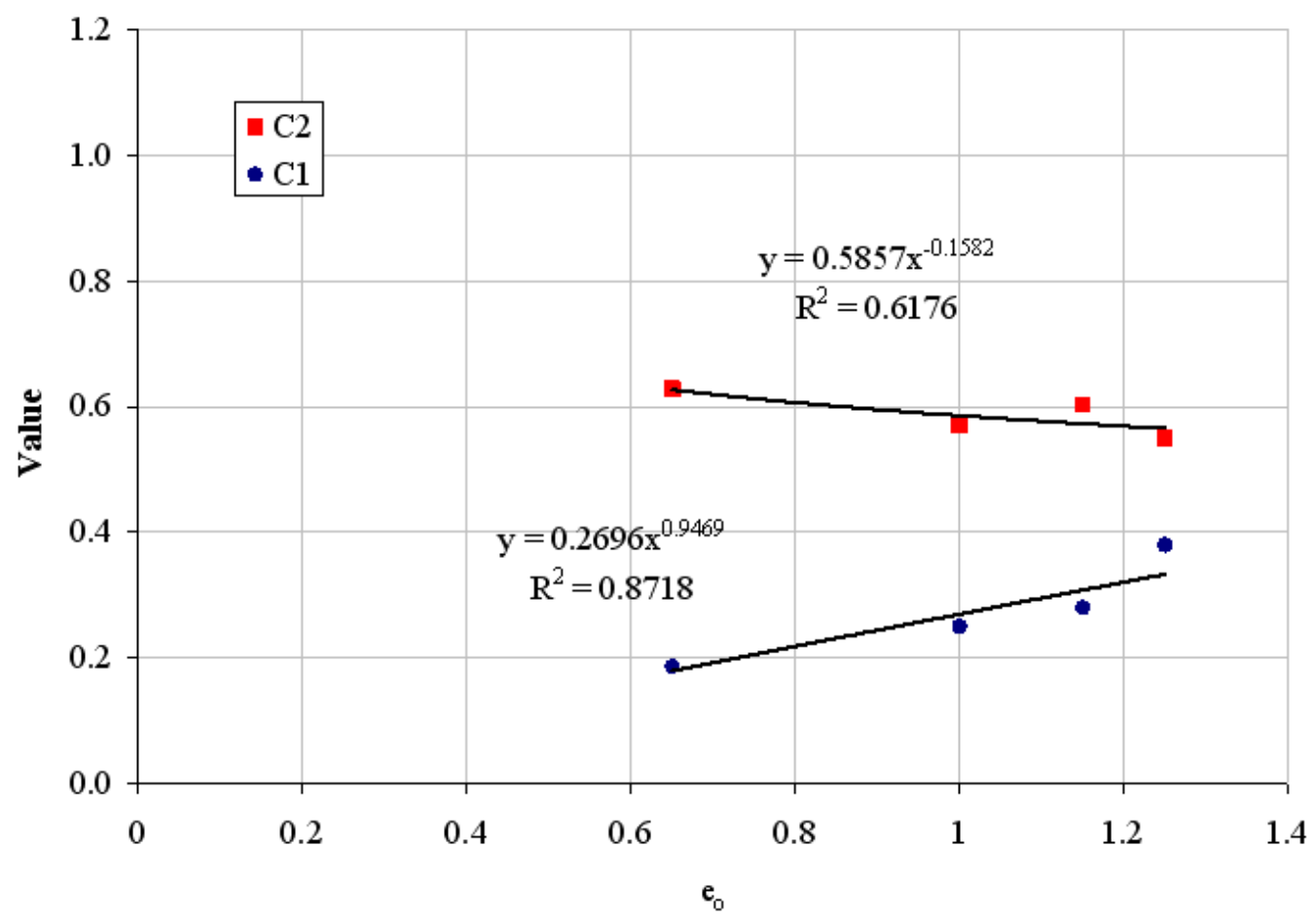

Figure 4-28 Variation of $\mathrm{C}_{1}$ and $\mathrm{C}_{2}$ with initial void ratio

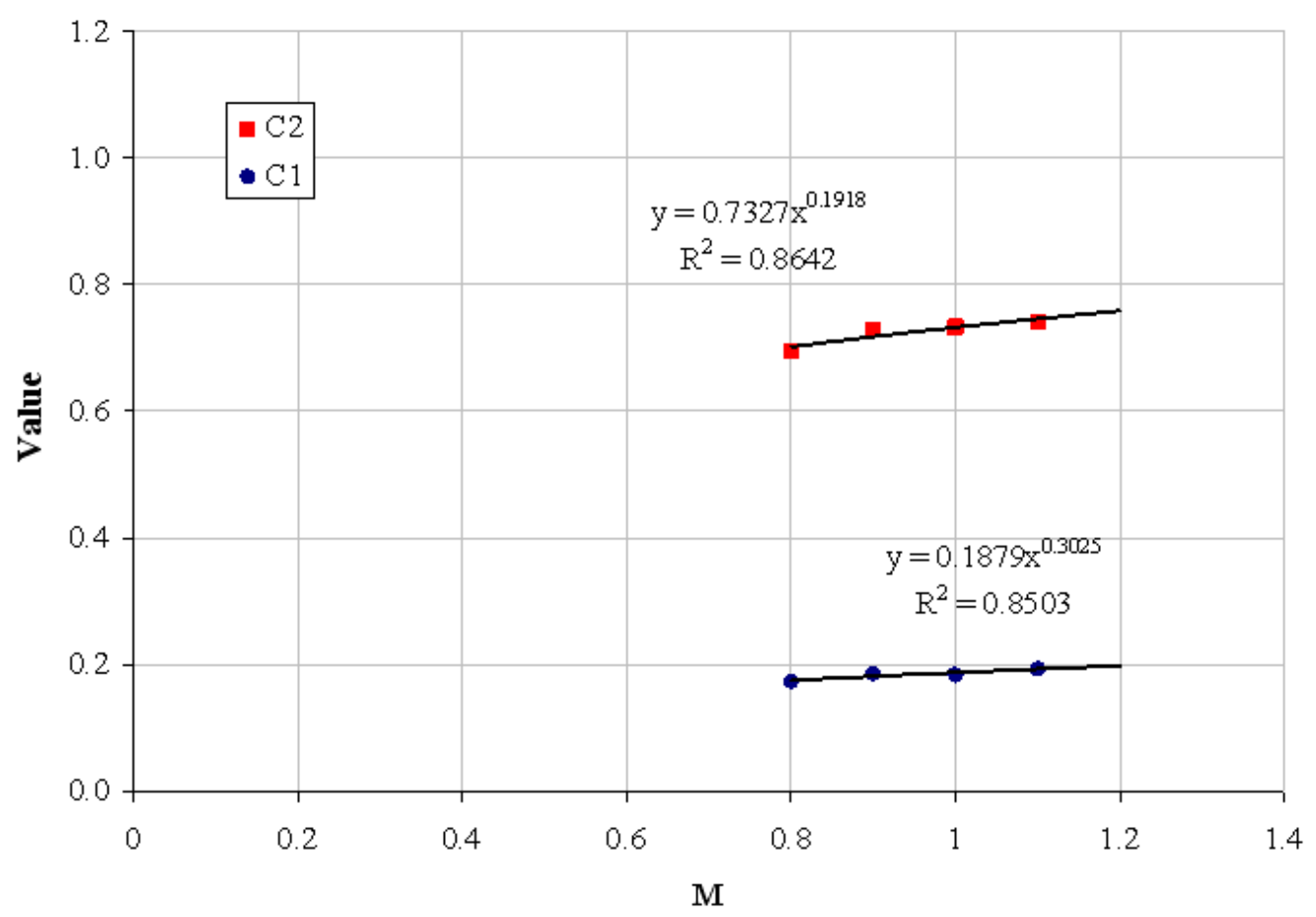

Figure 4-29 Variation of $\mathrm{C}_{1}$ and $\mathrm{C}_{2}$ with CSL slope (M) 


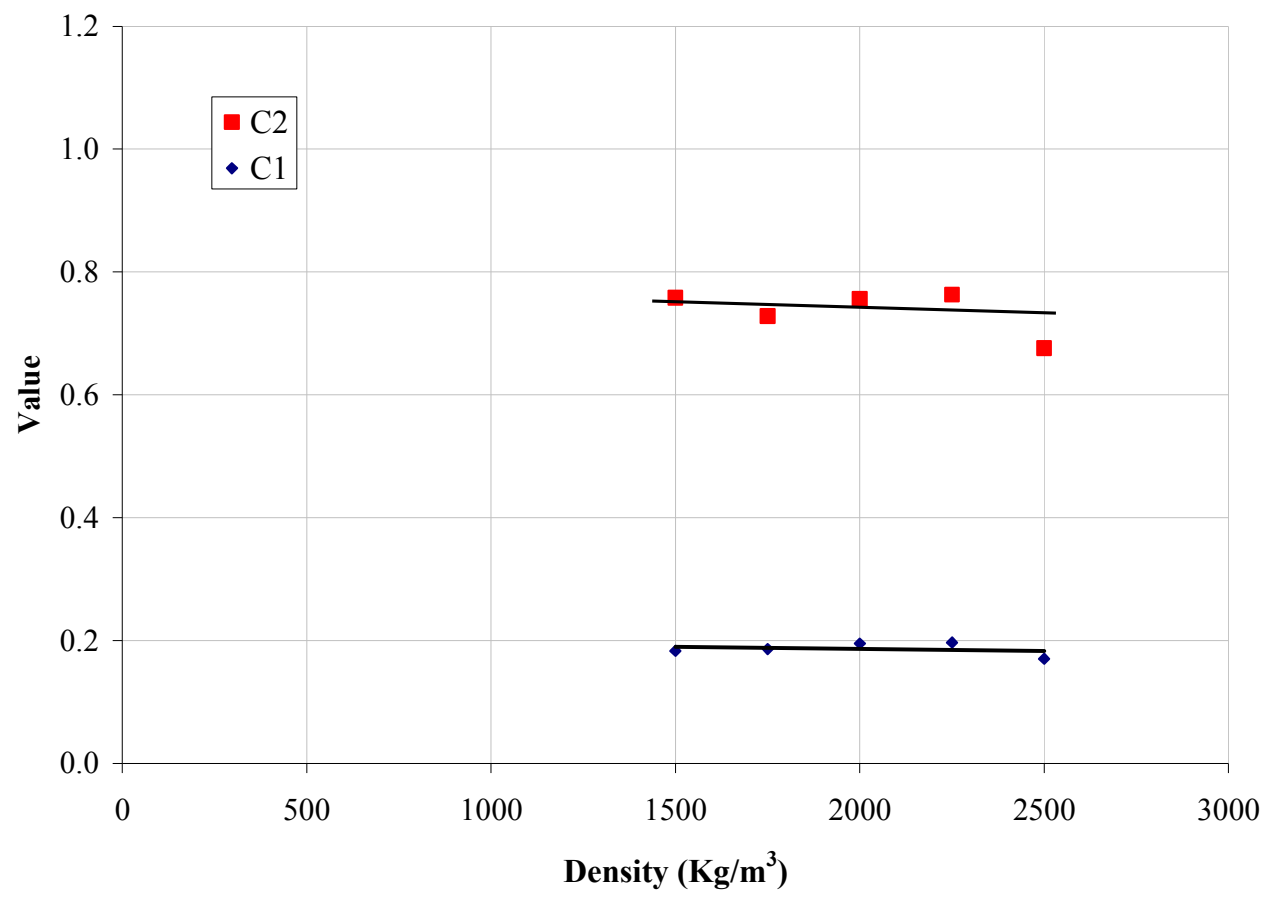

Figure 4-30 Variation of $\mathrm{C}_{1}$ and $\mathrm{C}_{2}$ with soil density

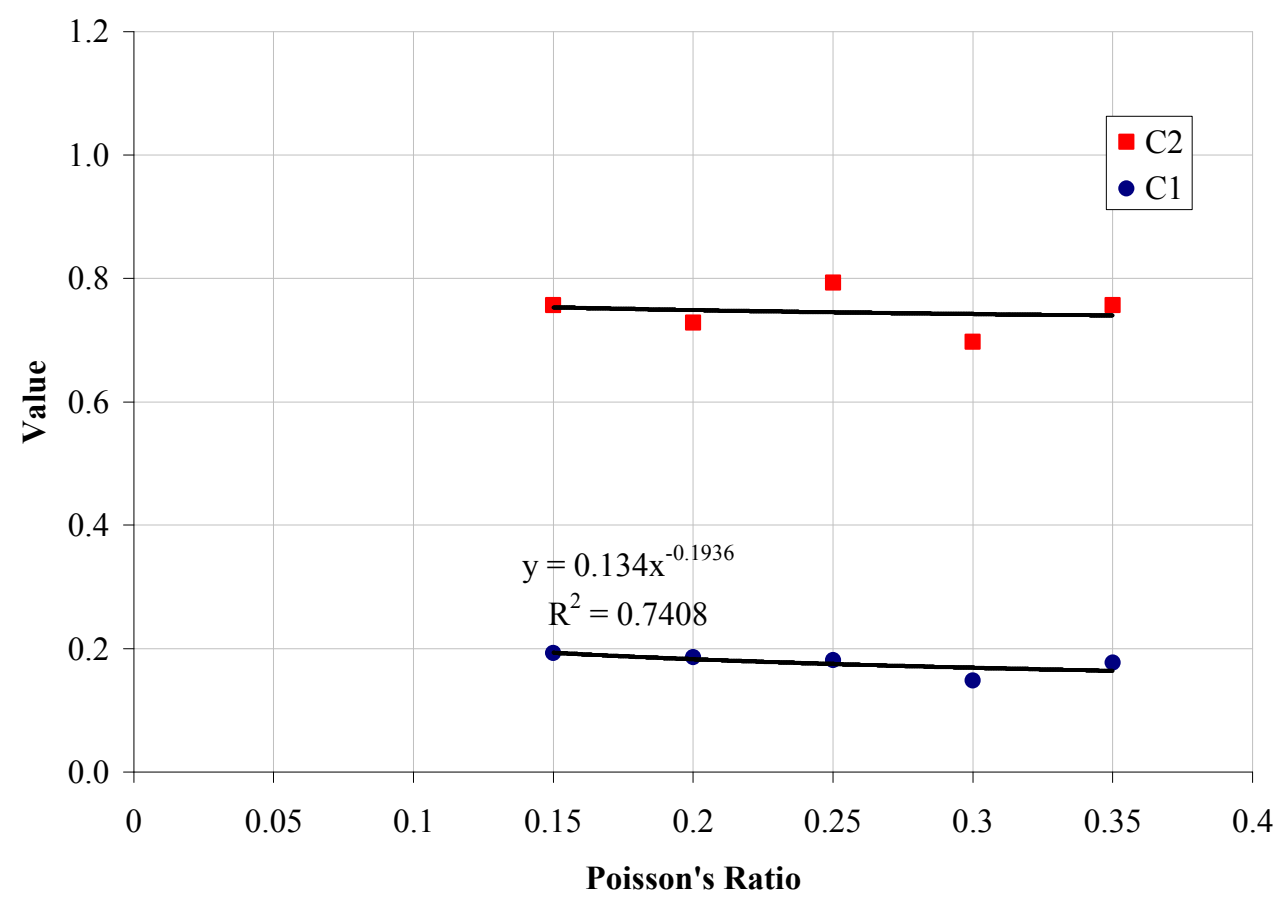

Figure 4-31 Variation of $C_{1}$ and $C_{2}$ with Poisson's ratio 


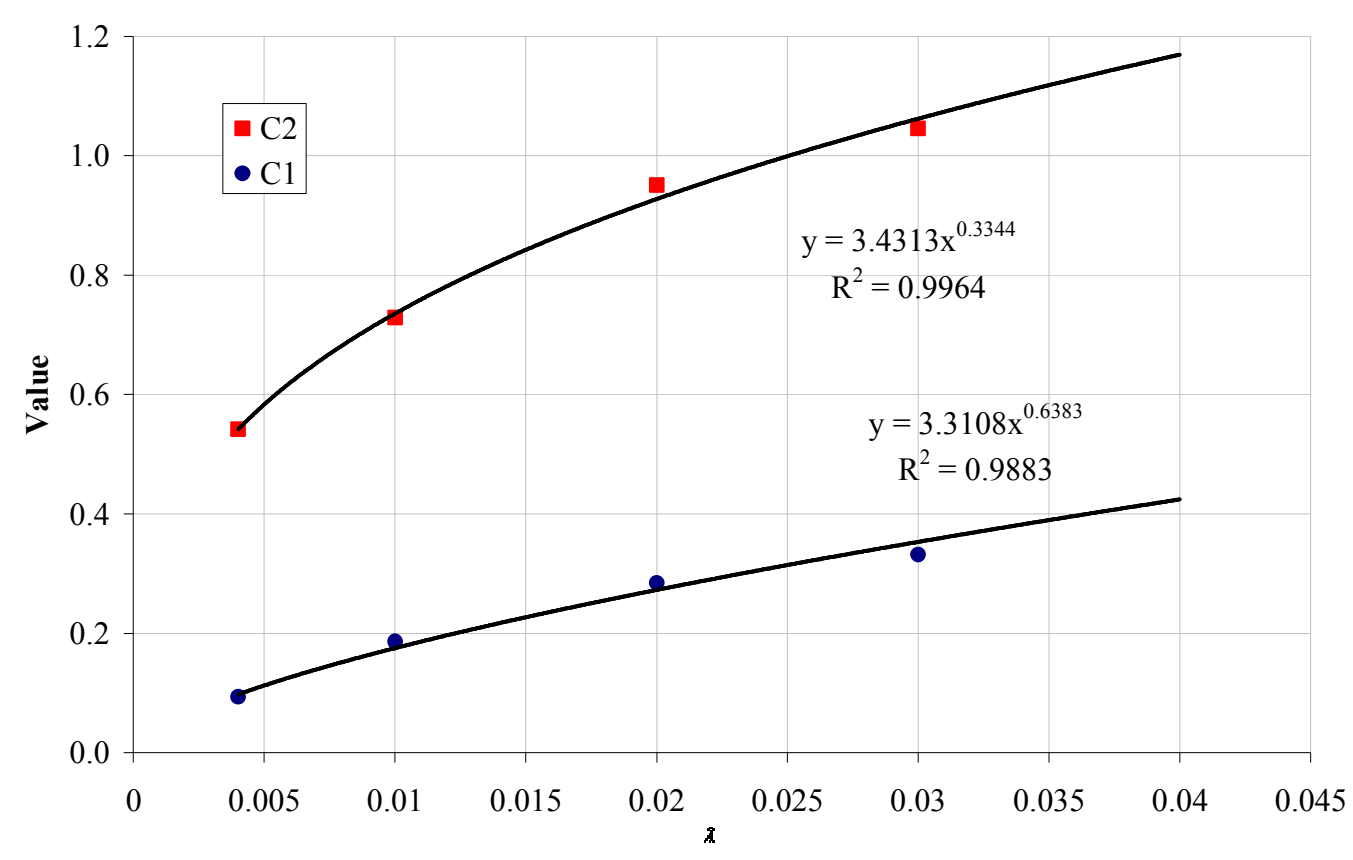

Figure 4-32 Variation of $C_{1}$ and $C_{2}$ with logarithmic plasticity bulk modulus $(\lambda)$

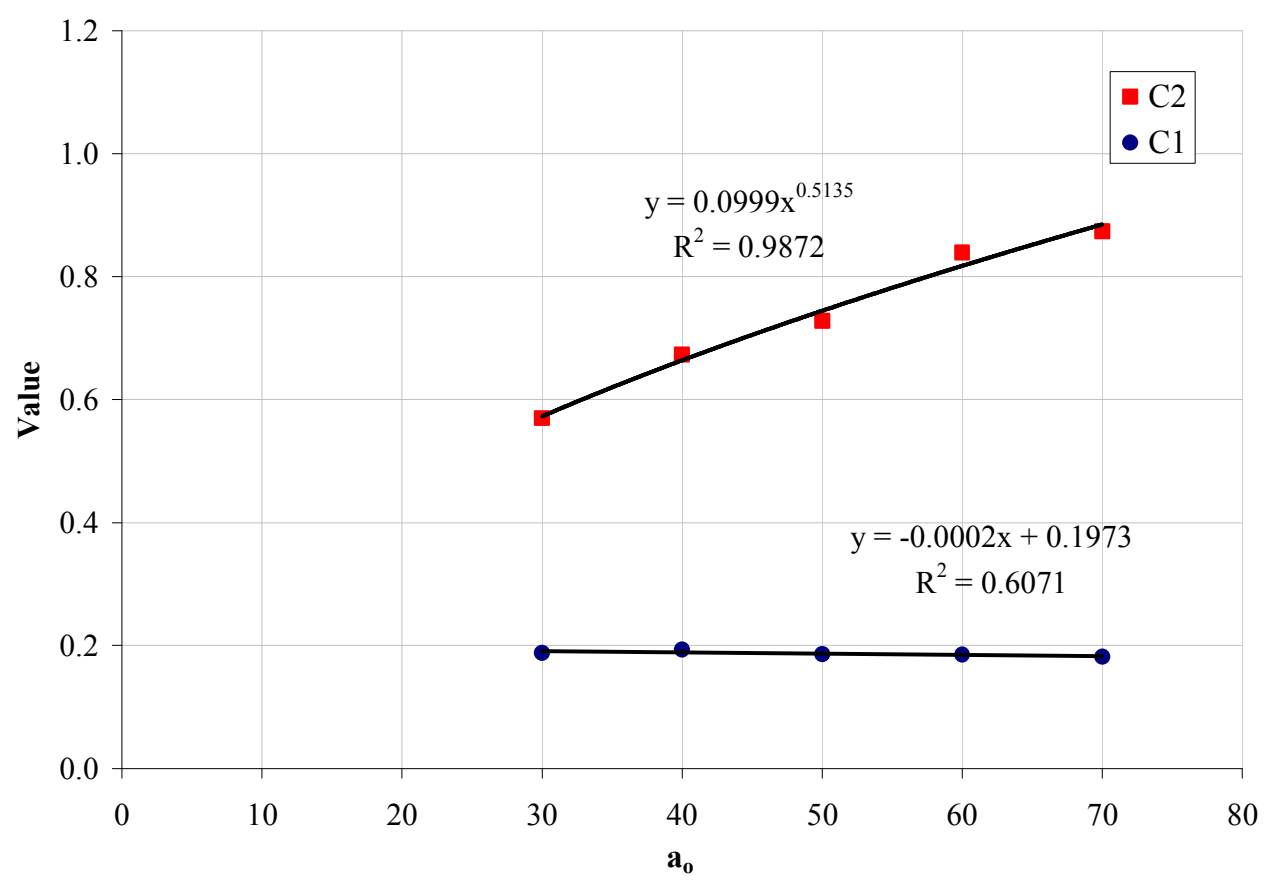

Figure 4-33 Variation of $\mathrm{C}_{1}$ and $\mathrm{C}_{2}$ with pre-consolidation pressure 
After obtaining the constant $\mathrm{C}_{1}$ and $\mathrm{C}_{2}$ using the developed MATLAB code, sensitivity analysis was conduced to check the importance of each soil parameter on $\mathrm{C}_{1}$ and $\mathrm{C}_{2}$. Table 4-4 and Table 4-5 summarize the sensitivity analysis results The same results shown in Table 4-4 and Table 4-5 are presented in Figure 4-34 as a bar chart. It can be seen that the order of importance of parameters affecting $\mathrm{C}_{1}$ can be ranked from high to low as follows: $\left(\lambda, e_{o}, v, M, \rho, a_{o}\right)$. It can be noticed also that the pre-consolidation pressure and soil initial density have exerted relatively small effect on $\mathrm{C}_{1}$. Therefore, it was not considered constant through out the subsequent statistical analysis to obtain empirical equation for $\mathrm{C}_{1}$. On the other hand, the order of parameters affecting $\mathrm{C}_{2}$ can be ranked from high to low as follows: $\left(\lambda, a_{o}, e_{o}, M, v, \rho\right)$. Poisson's ratio and initial density were excluded, since they have slight influence on $\mathrm{C}_{2}$.

Based on the correlation presented in the charts shown and using SPSS statistical analysis software, the following Equations are obtained.

$$
\begin{aligned}
C_{1} & =\frac{0.58 e_{o}^{1.4} \lambda^{0.9}}{M^{0.32} v^{0.2}} \\
C_{2} & =\frac{0.22 M^{.23} \lambda^{0.5} a_{o}^{0.5}}{e_{o}^{0.17}}
\end{aligned}
$$

Goodness of fit of the nonlinear regression analysis was examined using Rsquared when comparing the predicted and FE calculated $\mathrm{C}_{1}$ and $\mathrm{C}_{2}$ as shown in Figure 4-35 and Figure 4-36. The R-squared was found to equal 0.9608 for $C_{1}$ and 0.8250 for $C_{2}$. Based on the analysis presented, the two empirical equations are considered statistically reliable in the range of parameter used for the study. 
Table 4-4 Importance analysis for $\mathrm{C}_{1}$

\begin{tabular}{|l|c|c|c|c|c|c|}
\cline { 2 - 7 } \multicolumn{1}{c|}{} & $a_{o}$ & $\lambda$ & $v$ & $\rho$ & $M$ & $e_{0}$ \\
\hline Max & 0.1932 & 0.3318 & 0.1930 & 0.1968 & 0.1940 & 0.2656 \\
\hline Min & 0.1818 & 0.0936 & 0.1487 & 0.1775 & 0.1700 & 0.1072 \\
\hline Max- Min & 0.0114 & 0.2382 & 0.0444 & 0.0192 & 0.0240 & 0.1584 \\
\hline Max-Min/ $\sum$ (Max- Min) (\%) & $2 \%$ & $48 \%$ & $9 \%$ & $4 \%$ & $5 \%$ & $32 \%$ \\
\hline
\end{tabular}

Table 4-5 Importance analysis for $\mathrm{C}_{2}$

\begin{tabular}{|l|c|c|c|c|c|c|}
\cline { 2 - 7 } \multicolumn{1}{c|}{} & $a_{o}$ & $\lambda$ & $v$ & $\rho$ & $M$ & $e_{0}$ \\
\hline Max & 0.8738 & 1.0460 & 0.7929 & 0.7630 & 0.7417 & 0.7283 \\
\hline Min & 0.5699 & 0.5416 & 0.7725 & 0.7283 & 0.6760 & 0.5712 \\
\hline Max-Min & 0.3039 & 0.5044 & 0.0204 & 0.0347 & 0.0657 & 0.1571 \\
\hline Max-Min/ $\sum$ (Max- Min) (\%) & $28 \%$ & $46 \%$ & $2 \%$ & $3 \%$ & $6 \%$ & $14 \%$ \\
\hline
\end{tabular}

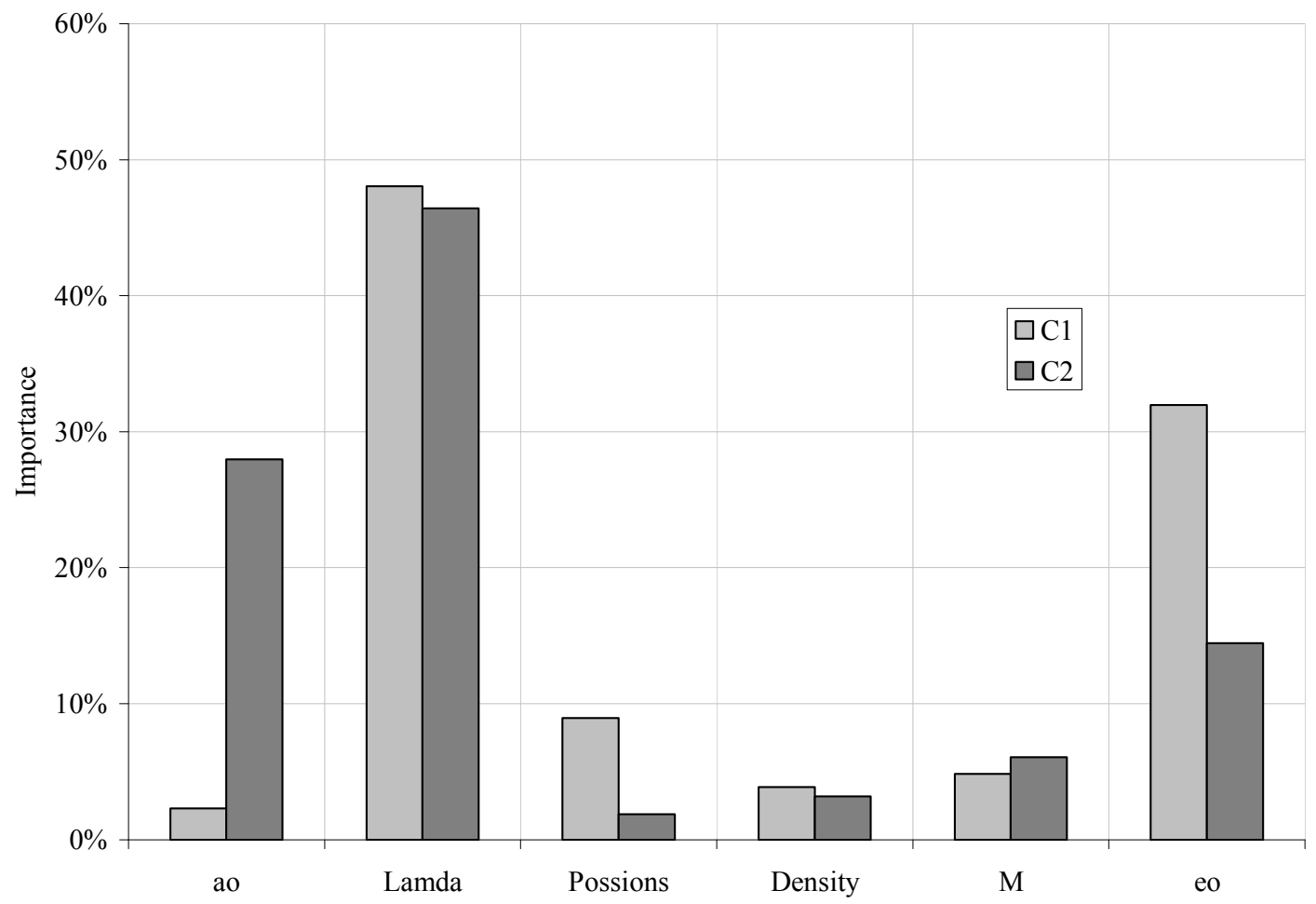

Figure 4-34 Importance analysis for $\mathrm{C}_{1}$ and $\mathrm{C}_{2}$ 


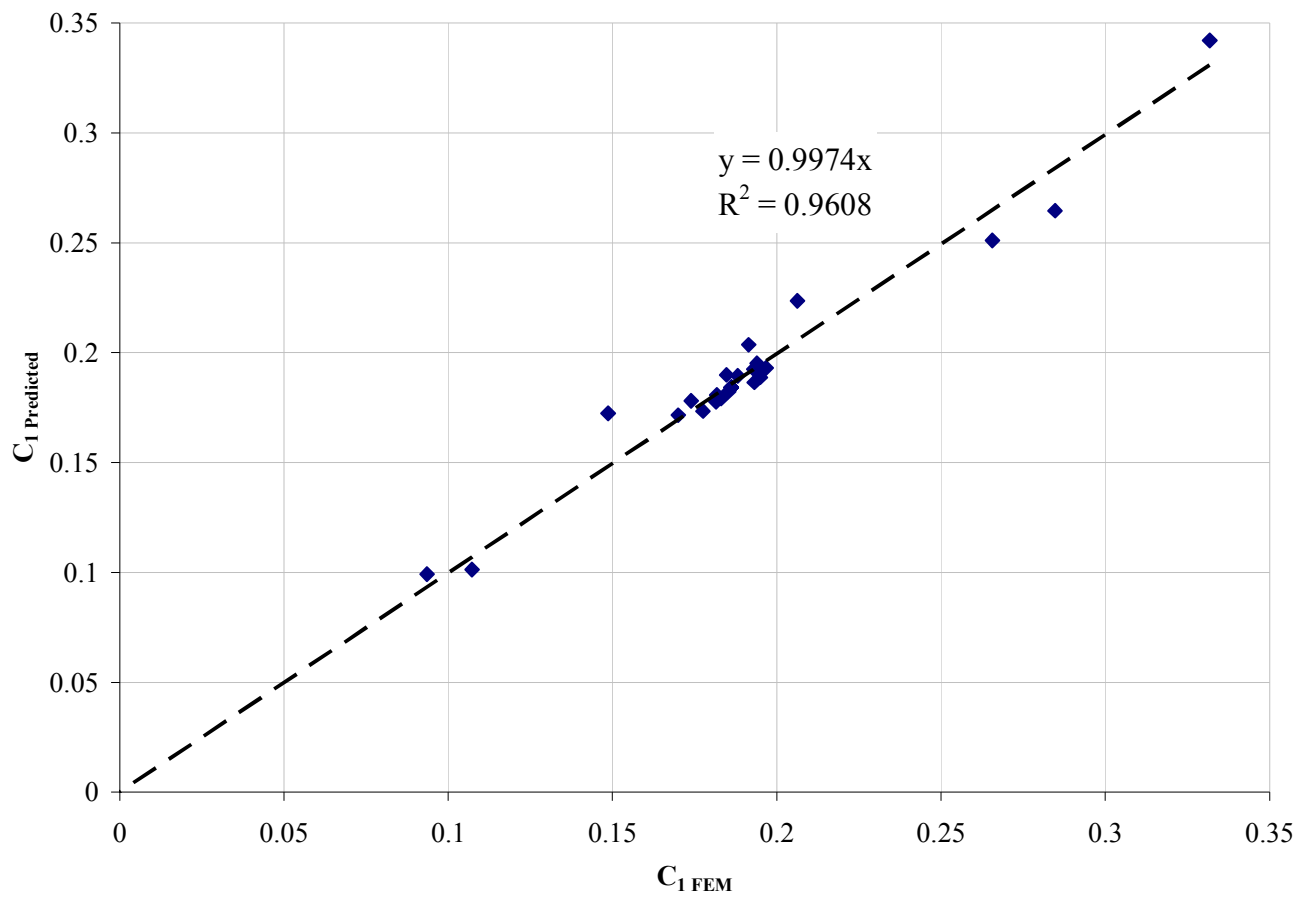

Figure 4-35 Model validation for parameter $\mathrm{C}_{1}$

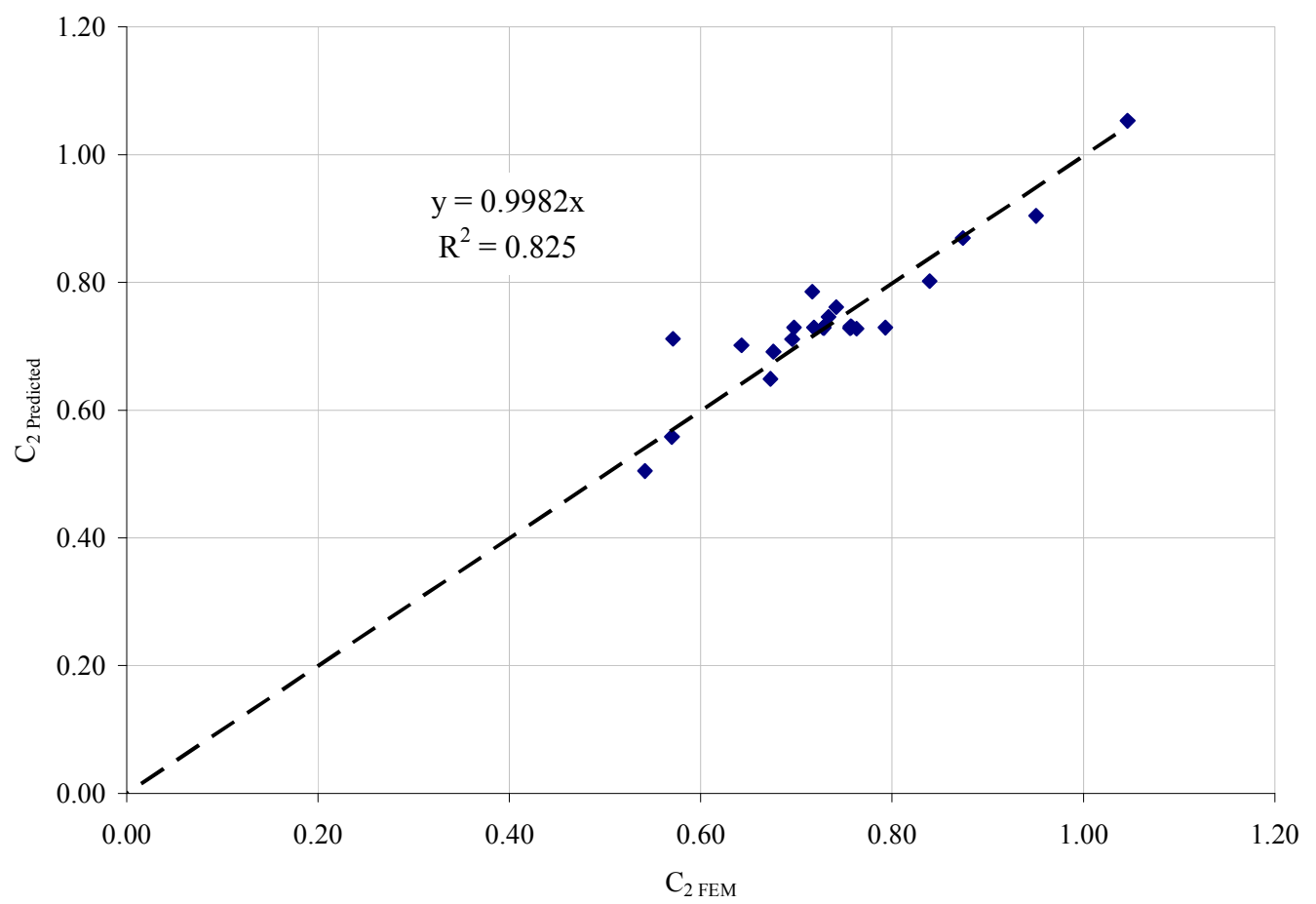

Figure 4-36 Model validation for parameter $\mathrm{C}_{2}$ 


\subsection{Peak Particle Velocity}

During DC, body and surface waves are generated due to the impact of the falling weight onto the ground surface. The generated waves would travel to the structures nearby and possibly could cause vibration induced damages on structures if the particle peak velocity exceeds a threshold value. In this section, analysis of FE model results of ground vibrations caused by DC was presented. The main objectives of this study are to investigate the influence of tamping parameters (e.g., energy per below) and Cam-Clay constitutive model parameters on the magnitude of Peak Particle Velocity (PPV).

The first step in the analysis is to investigate the influence of tamper parameters (i.e., tamper mass and drop height) on PPV. Figure 4-37 to Figure 4-38 show PPV along the ground surface for cases in which tamper mass and tamping velocity were varied while keeping soil properties to be constant as base line values listed previously in Chapter III. The horizontal axis represents distance measured from the center of the tamper. Close to the tamping location, disturbance in PPV magnitude is noticed. At 5 meter distance from the center of the tamper, the magnitude of the PPV decays exponentially. Comparisons between the magnitudes at the same distance indicated that tamping energy has its influence on PPV. For example, from Figure 4-37, the PPV was doubled at distance 20 meter as a result of increasing the tamper mass from 10 to 20 tons.

Figure 4-39 shows the variation of PPV with scaled tamping energy $\sqrt{W H} / A$ at three locations: at distance of 15, 20 and 30 meters from center of the tamper. Those locations were selected arbitrarily to estimate the PPV variations due to variations in the normalized energy. Using MS-Excel, the regression analysis shows that the PPV can be 
related to the scaled energy using a power function. Goodness of fit of the nonlinear regression analysis was examined using R-squared which was found to equal 0.9271, 0.9578 and 0.8027 for the correlations at distances 15,20 and 30 meters, respectively. Based on this regression analysis result, the following correlation was proposed to estimate the PPV as a function of scaled energy $\sqrt{W H} / A$, in which $\mathrm{B}_{1}$ and $\mathrm{B}_{2}$ are regression constants.

$$
P P V=B_{1}\left(\frac{\sqrt{W H}}{d}\right)^{B_{2}}
$$

The second step of the analysis investigates the influence of the Cam-Clay constitutive model parameters on the magnitude of PPV. Figure 4-40 to Figure 4-45 show the variation of PPV with each soil property. In this group of figures, soil properties were changed one at a time while keeping the rest of soil properties constant as those listed as baseline values in previous Chapter III. Also, tamping energy was kept as constant at 10 tones dropped from 10 meter height. The influence of each soil property on PPV velocity is noticed.

Figure 4-46 shows the summary for the PPV obtained from the FE simulations and presented in Figure 4-40 to Figure 4-45. The data was plotted on a log-log scale where the horizontal axis represents the scaled energy $\sqrt{W H} / d$ and the vertical axis represents the PPV in $\mathrm{m} / \mathrm{sec}$. Mayne (1985) models for estimating PPV were also replotted and superimposed on Figure 4-47. It can be seen that, FE simulations provide estimate of PPV which are close to those predicted by Mayne's model, especially for low tamping energy or at far field (low scaled energy $\sqrt{W H} / d$ ) 
Soil properties have huge influence on the PPV especially at the far field. Data summarized in Figure 4-46 show that the estimation for PPV falls between two lines that can be fitted into two correlations. The first correlation is considered a conservative estimation by fitting to the maximum PPV values. Of course, this is a very restrictive due to conservatism in its practical applications, as it may affect the decision of using DC to treat certain sites. The other correlation is a more aggressive (or non-conservative) which fits to the lower bound of the PPV values for all soil properties investigated. The two correlation are shown in Equations (4-7) and (4-8).

$$
\begin{gathered}
P P V(\mathrm{~m} / \mathrm{sec})=0.4\left(\frac{\sqrt{W H}}{d}\right)^{1.9} \\
P P V(\mathrm{~m} / \mathrm{sec})=0.18\left(\frac{\sqrt{W H}}{d}\right)^{2.2}
\end{gathered}
$$

To illustrate the significant difference between the two correlations, one can use 10 tons mass dropped from $10 \mathrm{~m}$ height as an example. The expected PPV can range from 20 to $60(\mathrm{~mm} / \mathrm{sec})$, as shown in Figure 4-47. This wide range of the estimated PPV results may stem from the wide variations in soil properties. Therefore, additional investigation needs to be done to correlate the constants $B_{1}$ and $B_{2}$ in Equation (4-6) to soil properties.

Using MATLAB curve fitting tool and using Equation (4-6) as a fitting model, the constants $\mathrm{B}_{1}$ and $\mathrm{B}_{2}$ were calculated for each case in which soil properties were changed while keeping the tamping energy constant. Figure 4-48 to Figure 4-54 show the variation of $\mathrm{B}_{1}$ and $\mathrm{B}_{2}$ with soil properties. The proposed correlation for each soil property is also 
shown on the Figure. R-squared is used to examine the goodness of fit for each proposed correlation.

The physical influence of $\mathrm{B}_{1}$ and $\mathrm{B}_{2}$ on the estimation of PPV can be explained from Equation (4-6). High value of the parameter $B_{1}$ indicates high PPV value at the same distance from the center of tamping for the same tamping energy. The parameter $\mathrm{B}_{2}$ has a several effects on of the estimation of PPV. High $\mathrm{B}_{2}$ value indicated higher value for PPV at the same distance. High $\mathrm{B}_{2}$ value also indicates higher decay of the PPV along the distance. This occurs as the PPV is inversely proportional to the distance measured from the center of tamping to the power $\mathrm{B}_{2}$.

Figure 4-48 shows the variation of $\mathrm{B}_{1}$ and $\mathrm{B}_{2}$ with initial void ratio. It can be noticed that increasing initial void ratio results in significant reduction in $\mathrm{B}_{1}$ value and increase in $\mathrm{B}_{2}$ value. Following the same procedure, two power functions were proposed to estimate $B_{1}$ and $B_{2}$. R-squared values were found to be equal to 0.8523 and 0.9736 for $B_{1}$ and $B_{2}$, respectively. The influence of the slope of critical state line (CSL) value (M) is shown in Figure 4-49. Both $B_{1}$ and $B_{2}$ increase with increasing M. R-squared values of the empirical equation were found to be equal to 0.9862 and 0.9424 for $B_{1}$ and $B_{2}$, respectively.

The influence of Poisson's ratio is shown in Figure 4-50. Similarly, two power functions were proposed to estimate PPV as a function of Poisson's ratio. R-squared fitting values were found to be equal to 0.8989 and 0.9618 for $\mathrm{B}_{1}$ and $\mathrm{B}_{2}$, respectively. Figure 4-51 shows the variation of $\mathrm{B}_{1}$ and $\mathrm{B}_{2}$ with the pre-consolidation pressure. The proposed correlation and their corresponding R-squared values are plotted on the same figure. The logarithmic plasticity bulk modulus influence $(\lambda)$ on the parameter $B_{1}$ and $B_{2}$ 
is shown in Figure 4-53. Increasing $\lambda$ results in significant decrease in $B_{1}$ value. A slight change in $B_{2}$ is noticed due to change in $\lambda$. Finally, the influence of soil density of the estimation of PPV is shown in Figure 4-53. Similarly two power functions were proposed for correlation, the corresponding R-squared values for $\mathrm{B}_{1}$ and $\mathrm{B}_{2}$ are 0.9551 and 0.9867, respectively. Table 4-6 and Table 4-7 summarize the parametric sensitivity analysis results, in which the constants $B_{1}$ and $B_{2}$ were calculated for each PPV profile obtained from the parametric study. The same results shown in Table 4-6 and Table 4-7 are presented in Figure 4-54 as a bar chart. It can be seen that plasticity bulk modulus $(\lambda)$ has the most significant effect on $\mathrm{B}_{1}$. The rest of parameters have similar influence on $\mathrm{B}_{1}$. On the other hand, the pre-consolidation pressure has the most significant effect on $\mathrm{B}_{2}$.

The results for $\mathrm{B}_{1}$ and $\mathrm{B}_{2}$ are analyzed by using a statistical analysis software SPSS (Statistical Package for the Social Sciences, 2003) program. It was assumed for the purpose of nonlinear regression, that $\mathrm{B}_{1}$ and $\mathrm{B}_{2}$ could be computed as in Equation (4-9) and (4-10) by a product of various functions. Each of those functions represents the variation of the parameter with one of the soil properties. The correlation is given in Equations 4-11 and 4-12 for parameters $\mathrm{B}_{1}$ and $\mathrm{B}_{2}$, respectively.

$$
\begin{aligned}
& B_{1}=g_{1}(\lambda) g_{2}\left(e_{o}\right) g_{3}(\rho) g_{4}(M) g_{5}\left(a_{o}\right) \\
& B_{2}=h_{1}(\lambda) h_{2}\left(e_{o}\right) h_{3}(\rho) h_{4}(M) h_{5}\left(a_{o}\right) \\
& B_{1}=\frac{16 v^{0.26} M a_{o}^{0.74} \lambda^{0.2}}{\rho^{0.85} e_{0}^{1.75}} \\
& B_{2}=\frac{0.66 \rho^{0.18} v^{0.08} M^{0.32} e_{o}^{0.06} \lambda^{0.05}}{a_{0}^{0.04}}
\end{aligned}
$$


Validation of $B_{1}$ and $B_{2}$ is shown in Figure (4-55) and Figure (4-56), respectively. The horizontal axis represents the value as calculated from MATLAB curve fitting tool for the data obtained from FEM simulations. The vertical axis represents the value of $\mathrm{B}_{1}$ and $\mathrm{B}_{2}$ calculated from Equations (4-11) and (4-12), respectively. Goodness of fit of the nonlinear regression analysis was examined using R-squared. The R-squared was found to equal 0.9256 and 0.8536 for $\mathrm{B}_{1}$ and $\mathrm{B}_{2}$, respectively. Based on the analysis presented, the formula is considered statistically reliable in the range of parameter values used for the study.

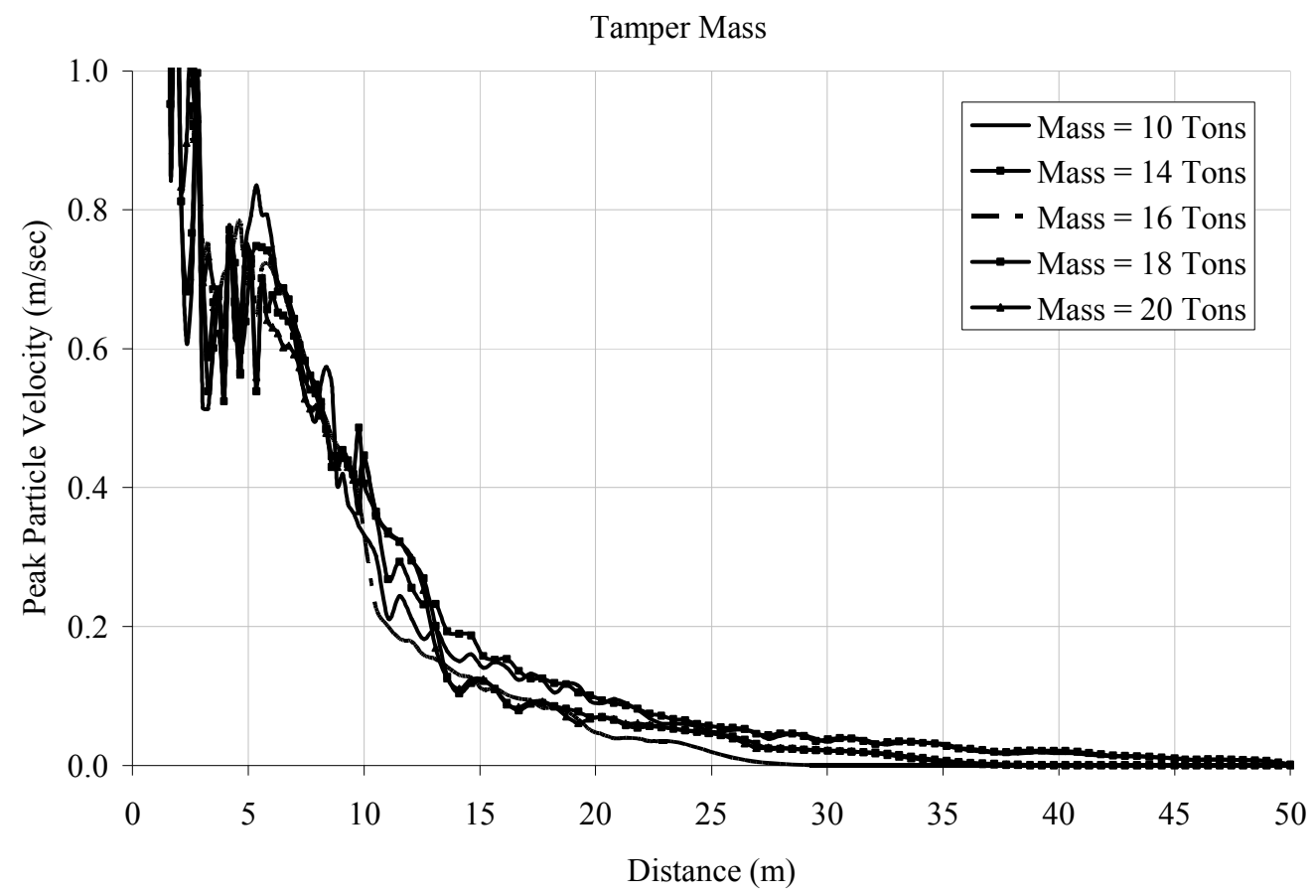

Figure 4-37 Variation of PPV with distance for different tamper mass dropped from 10 meter height 


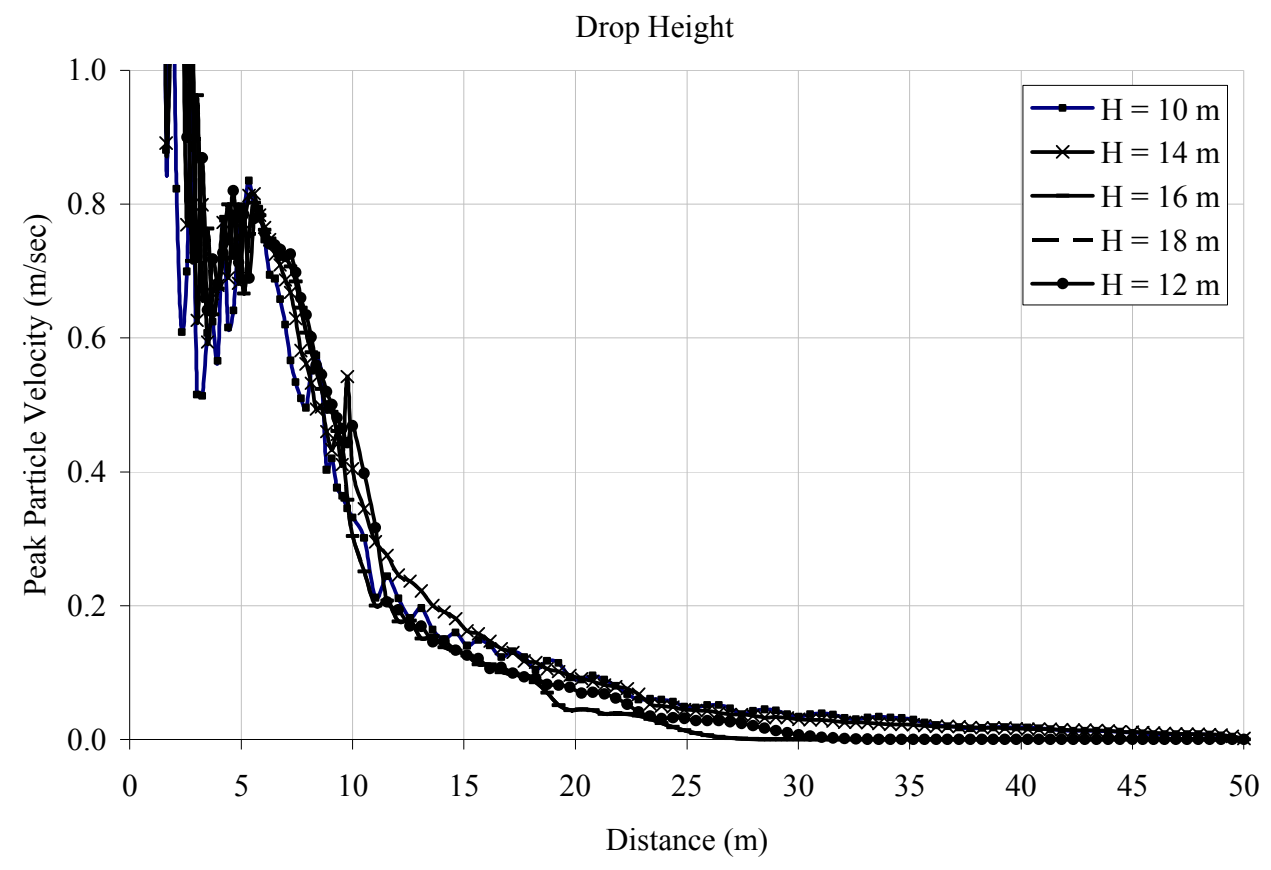

Figure 4-38 Variation of PPV with distance for different drop height using 10 tons tamper mass

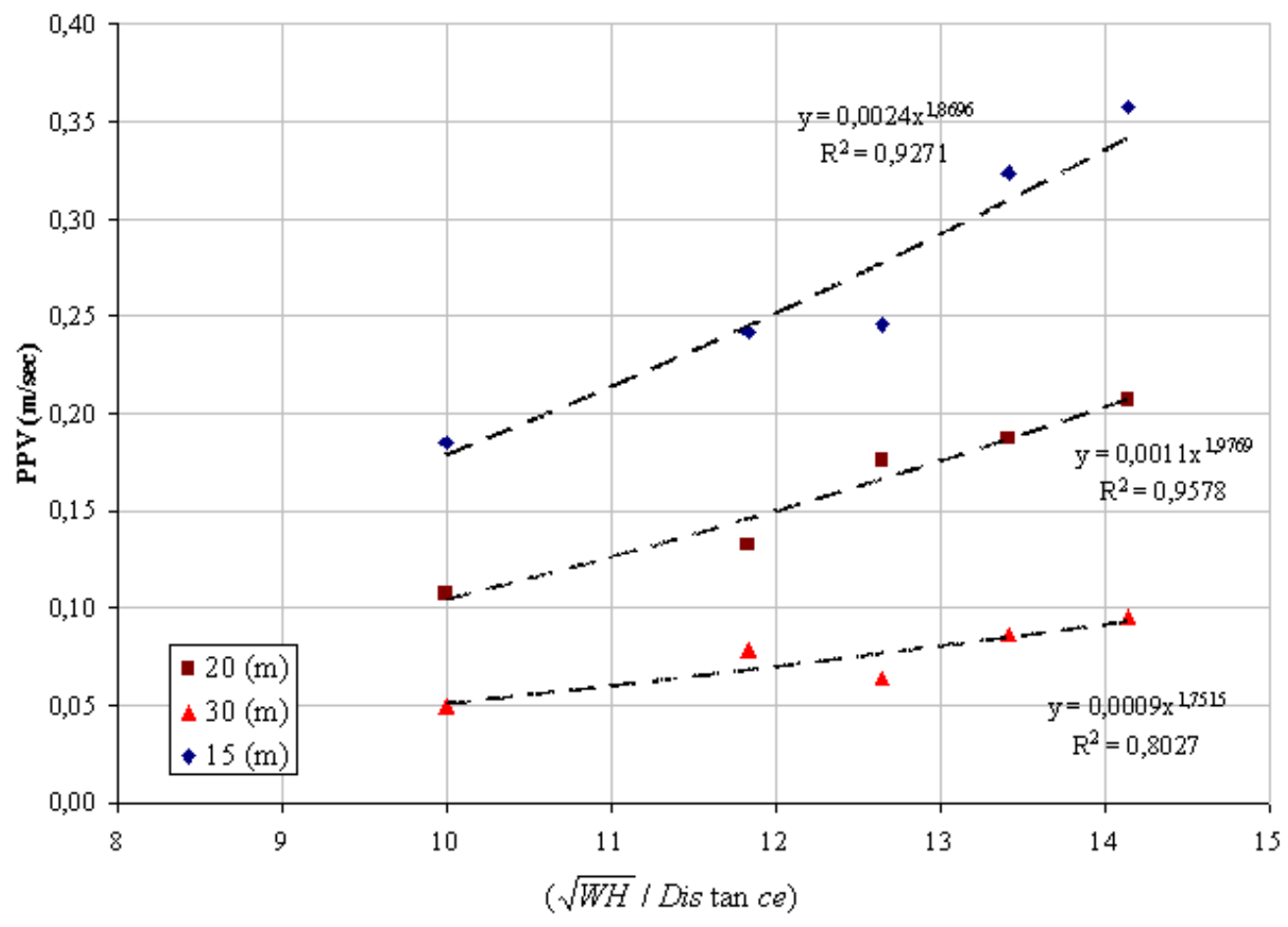

Figure 4-39 Variation of PPV with energy 


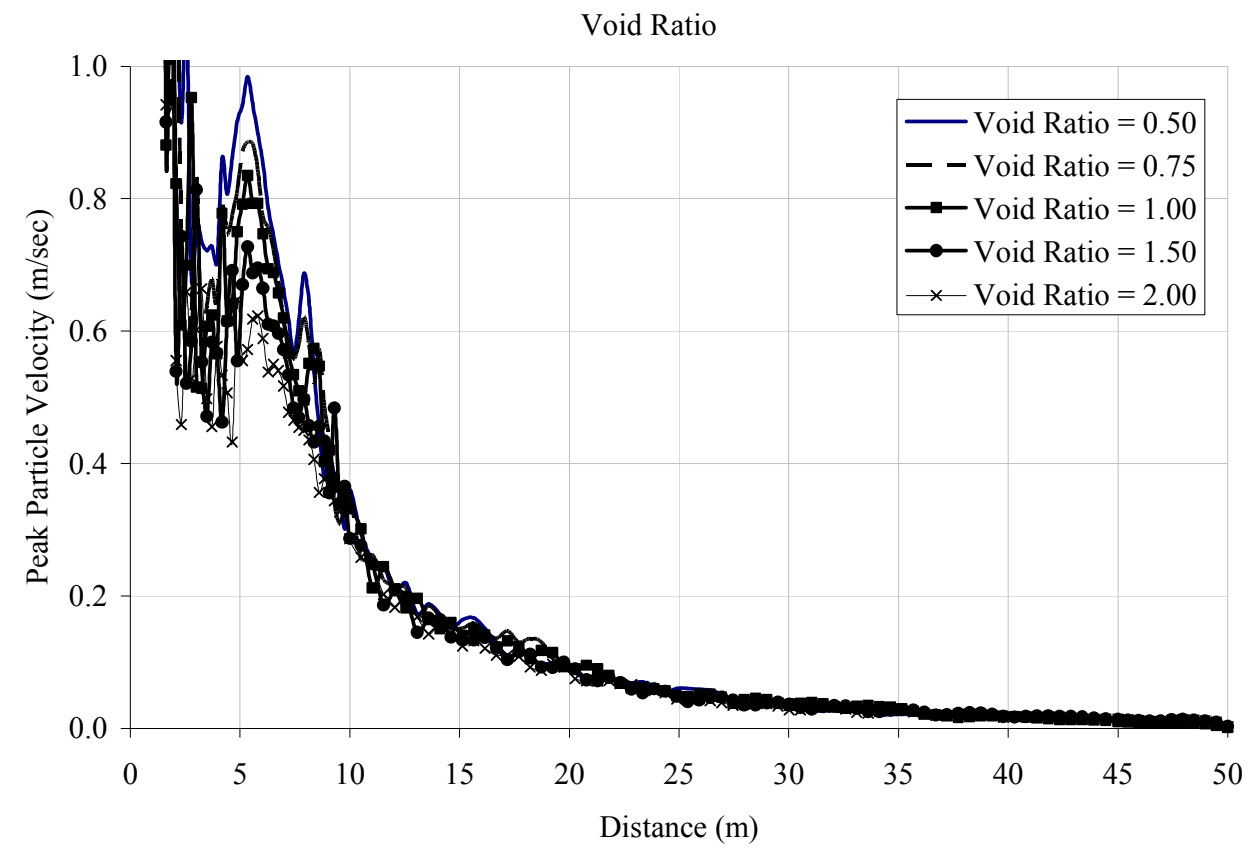

Figure 4-40 Variation of PPV with distance for different initial void ratio

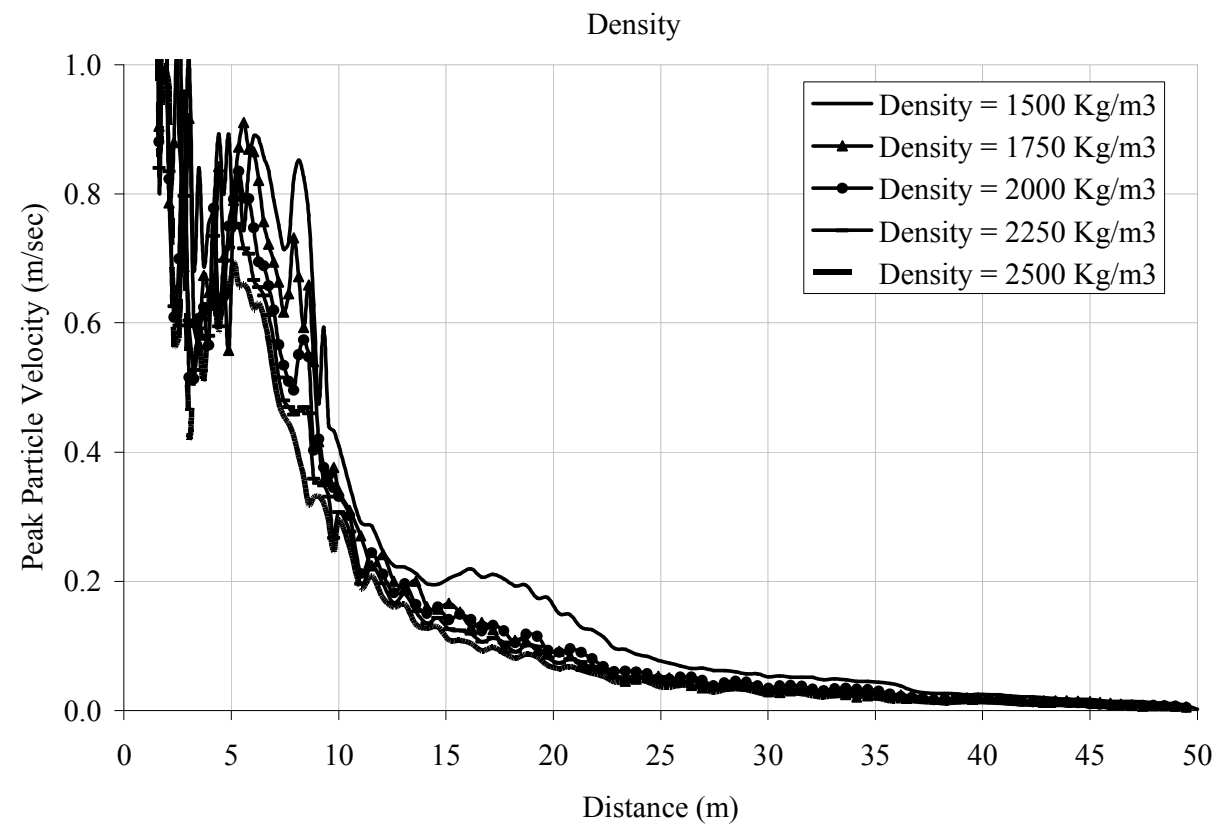

Figure 4-41 Variation of PPV with distance for different initial soil density 


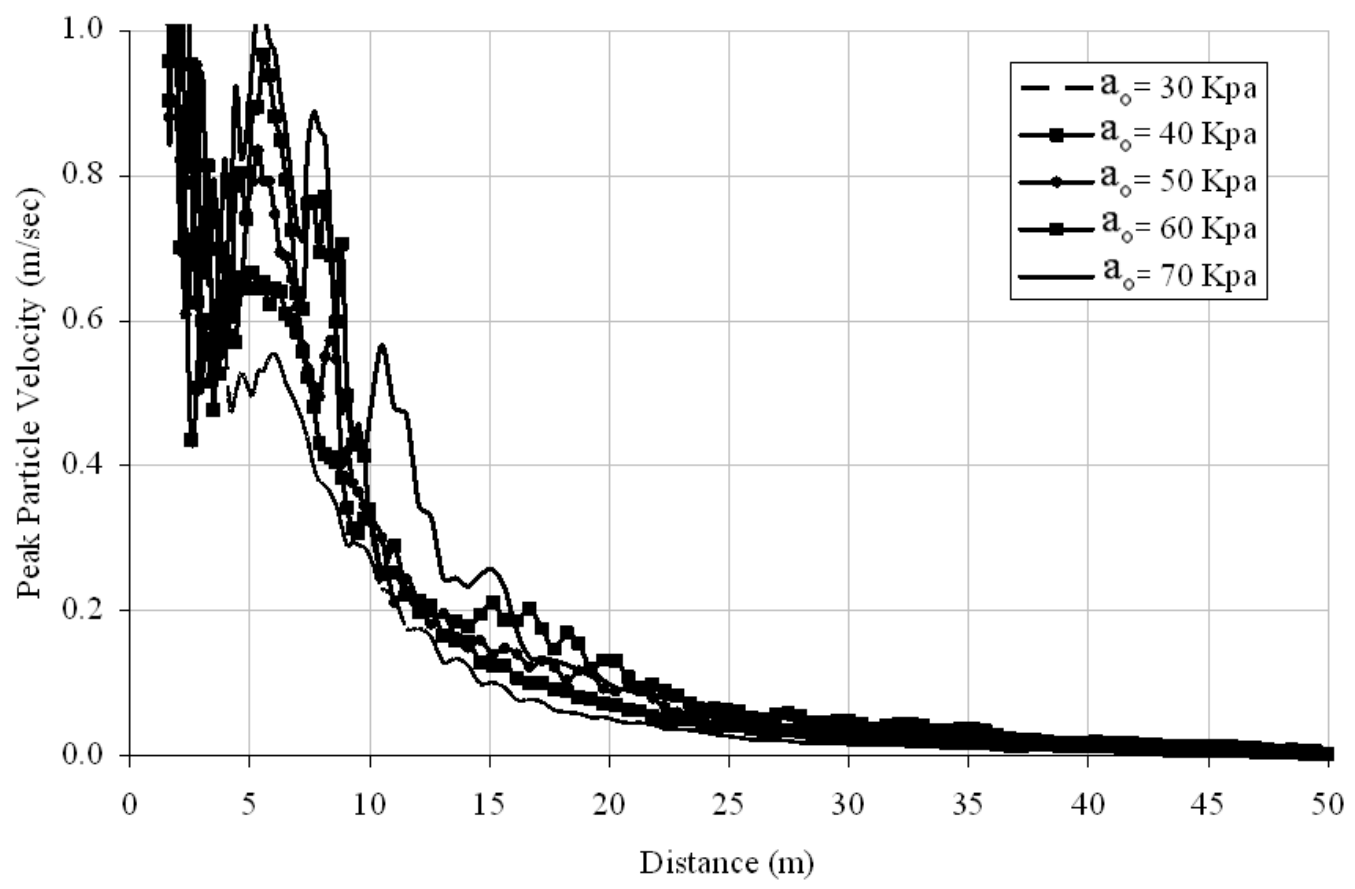

Figure 4-42 Variation of PPV with distance for different pre-consolidation pressure

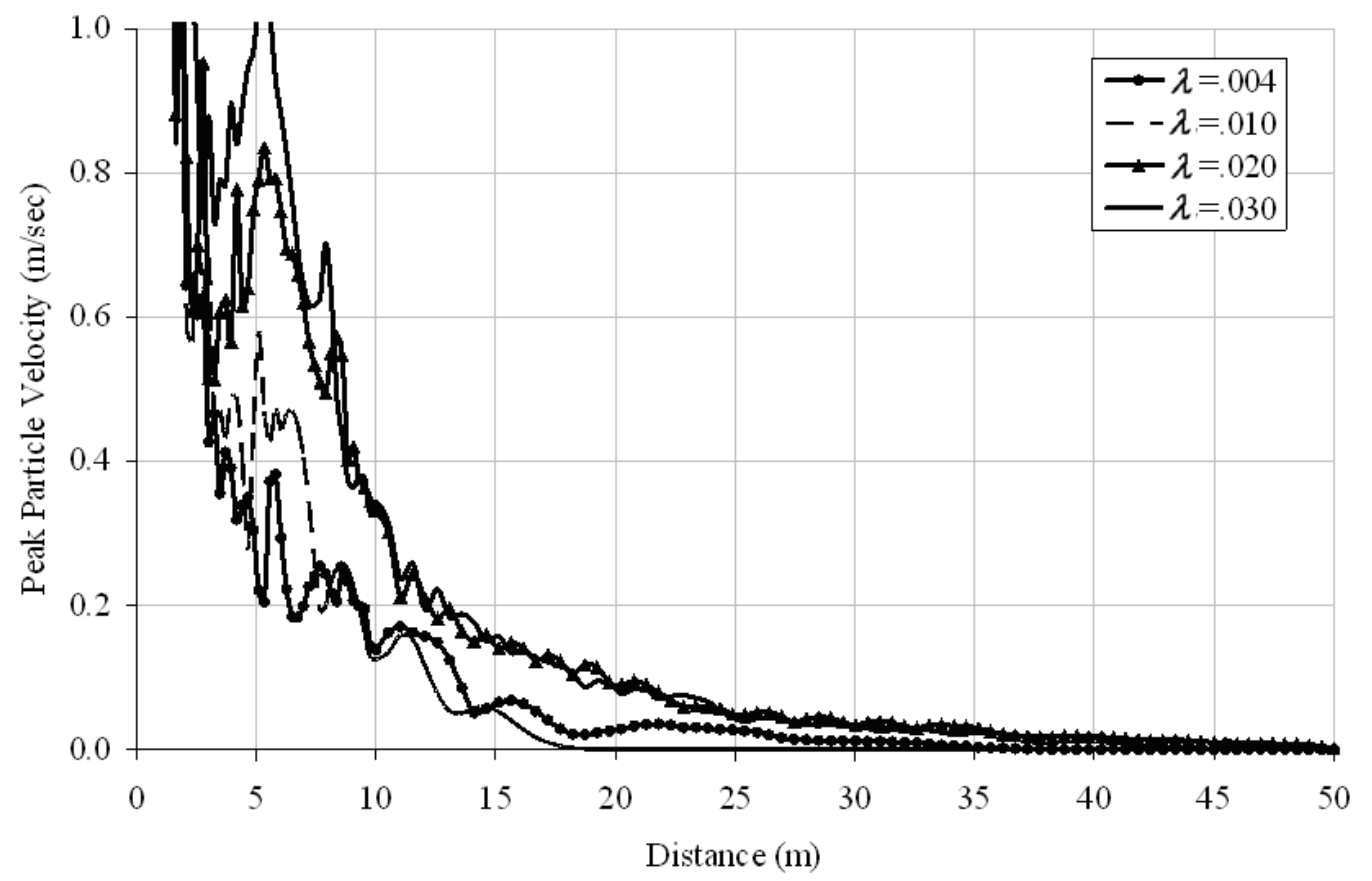

Figure 4-43 Variation of PPV with distance for different $\lambda$ values 


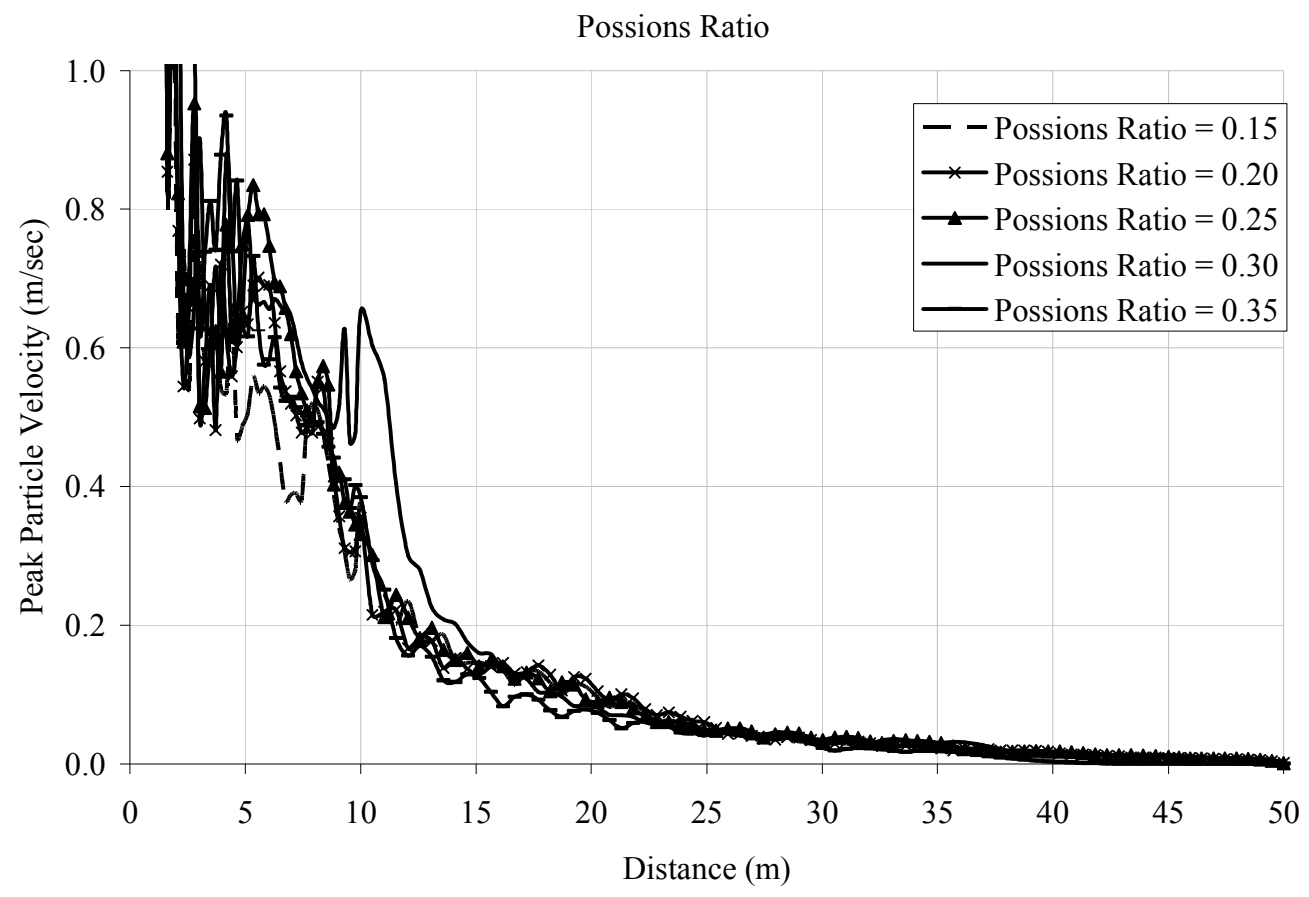

Figure 4-44 Variation of PPV with distance for different Poisson's ratio

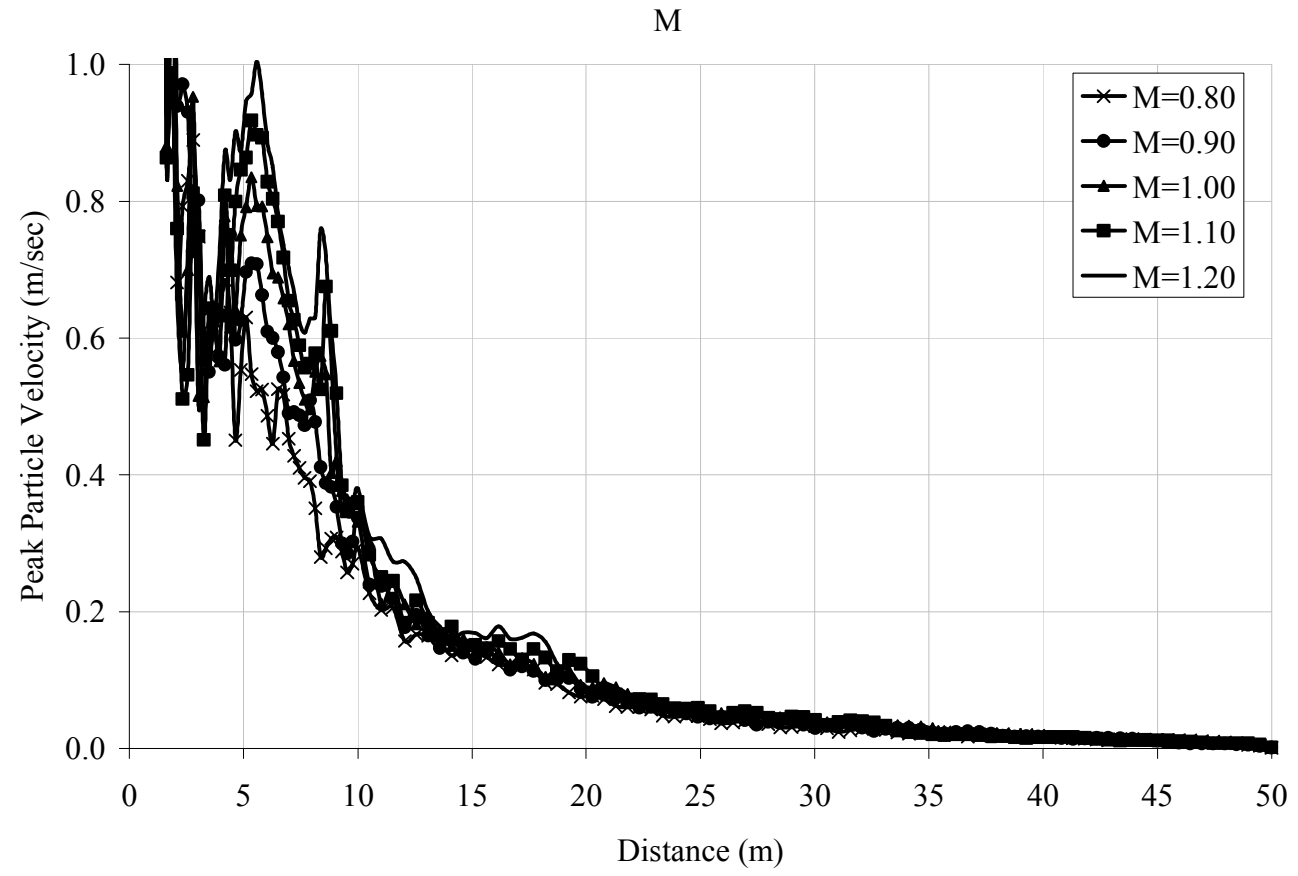

Figure 4-45 Variation of PPV with distance for different CSL slope (M) 


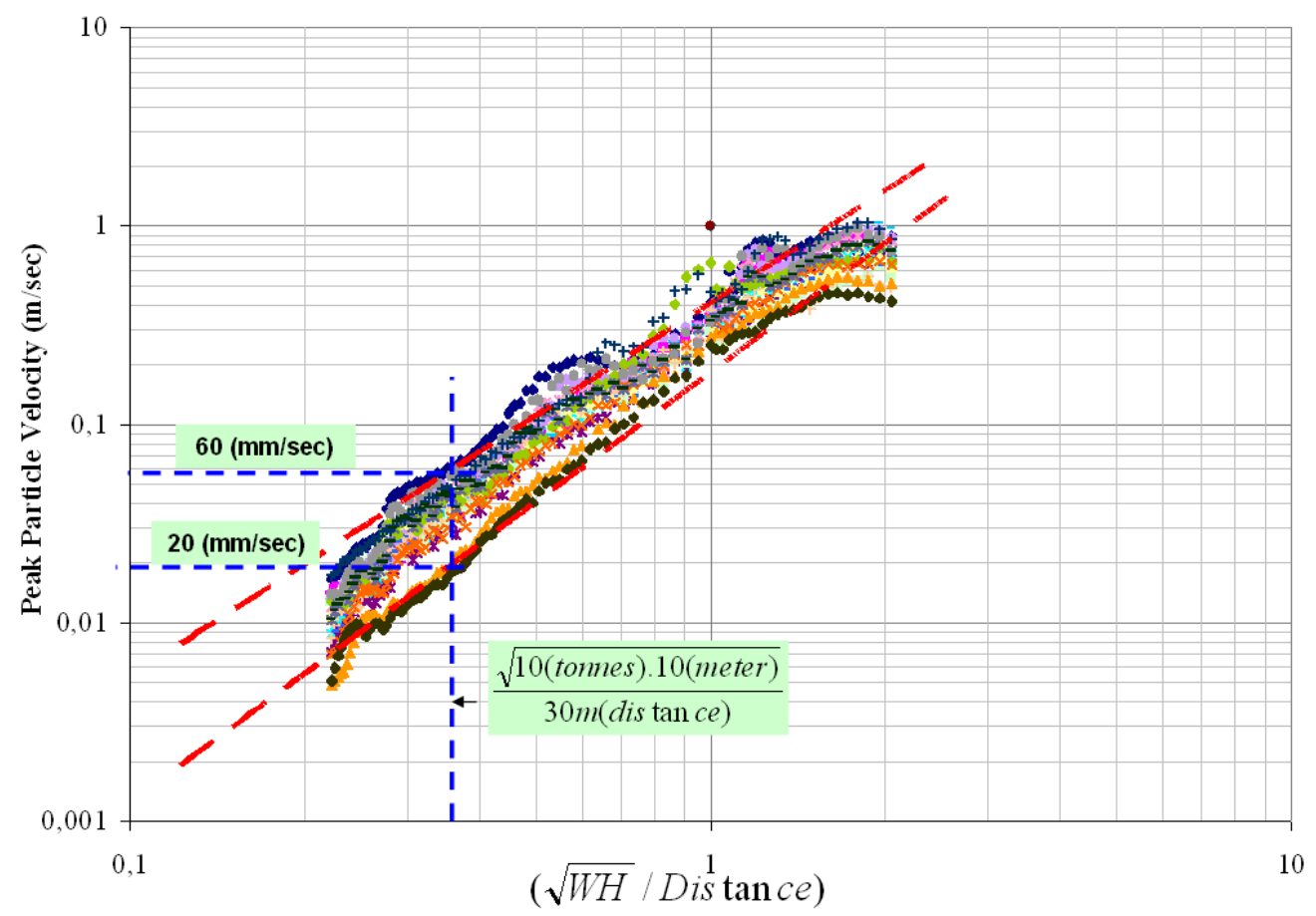

Figure 4-46 Variation of PPV with soil properties (FE results)

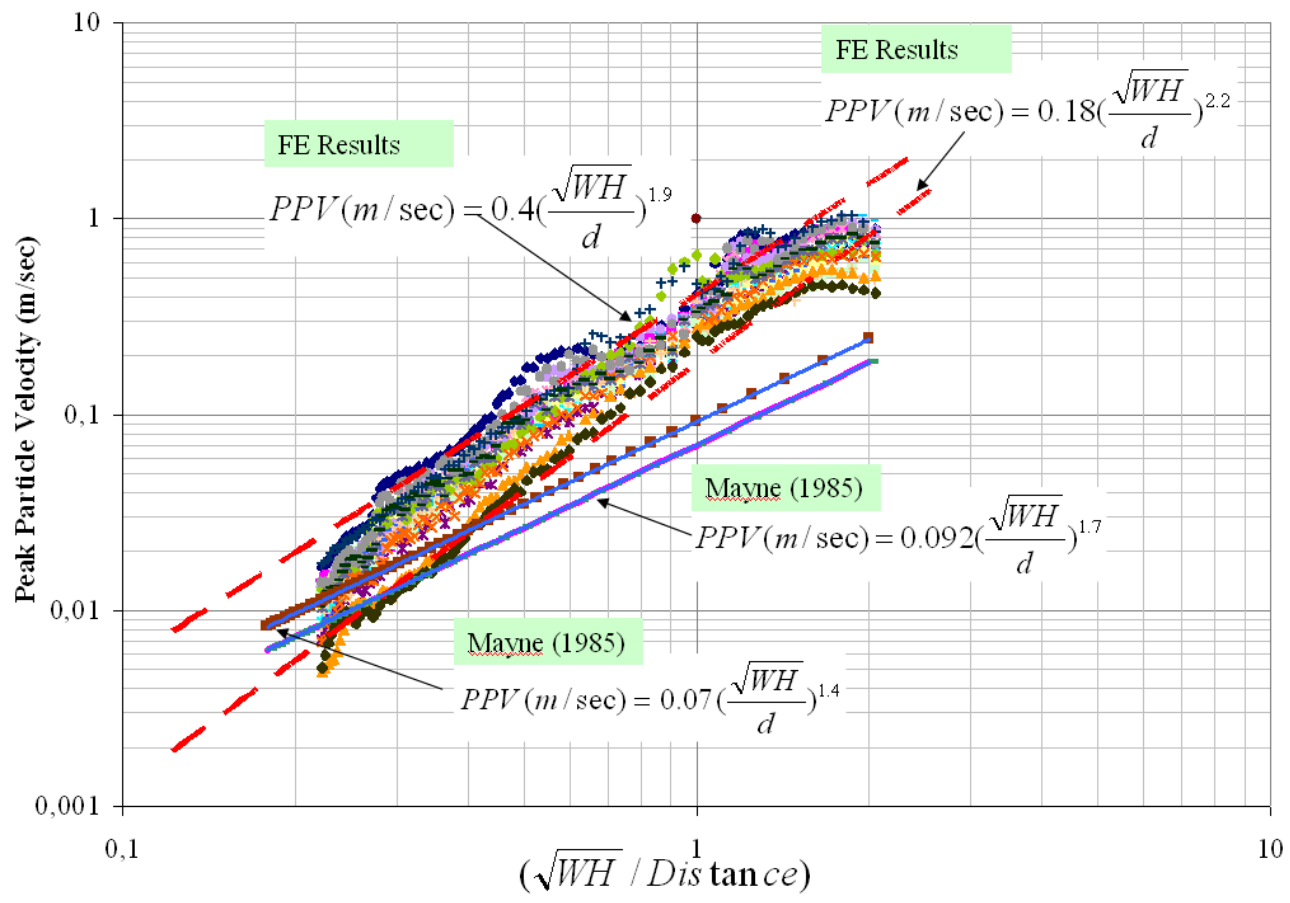

Figure 4-47 Variation of PPV with soil properties (both Fe and Mayne model) 


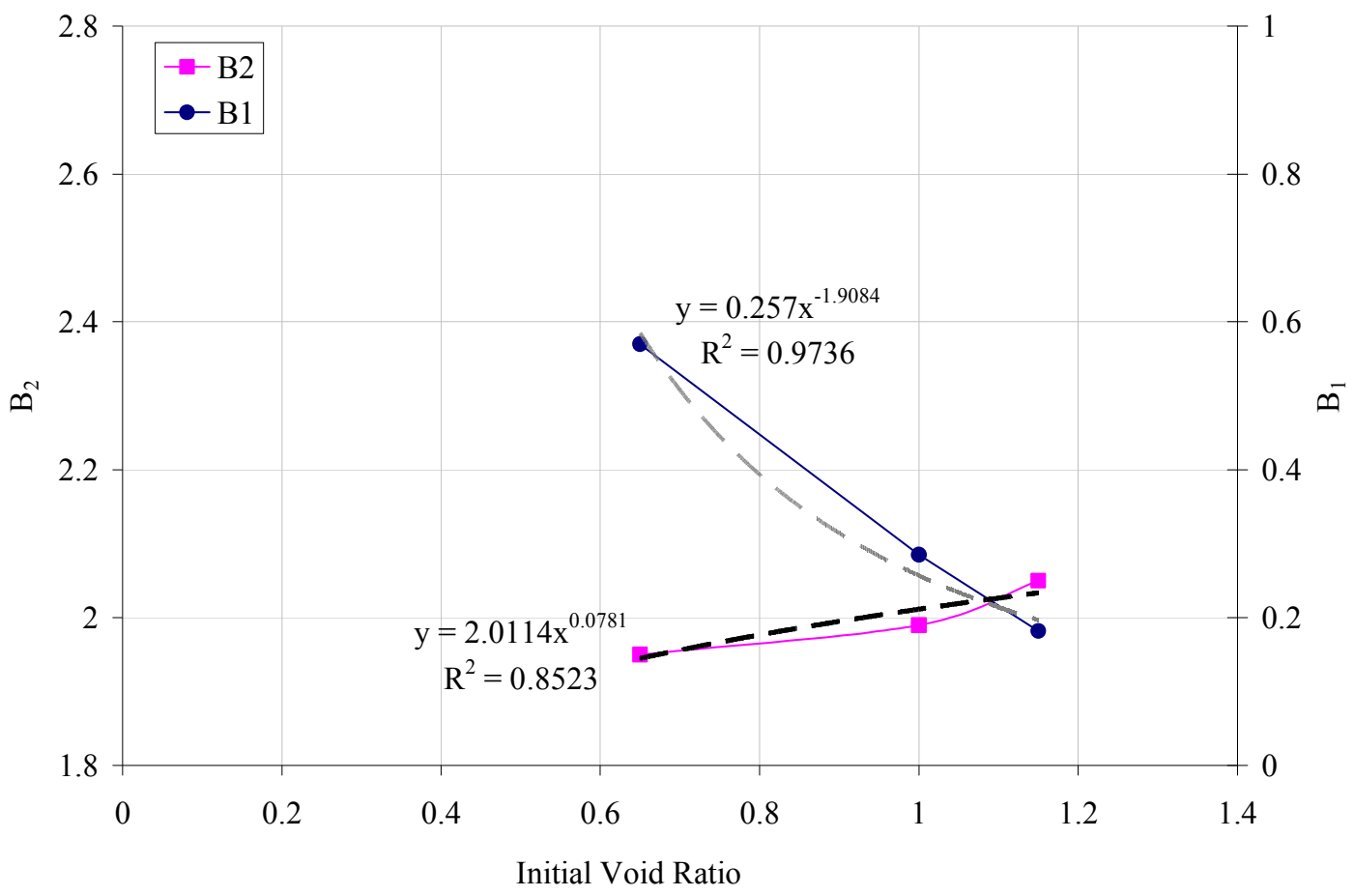

Figure 4-48 Variation of $\mathrm{B}_{1}$ and $\mathrm{B}_{2}$ with initial void ratio

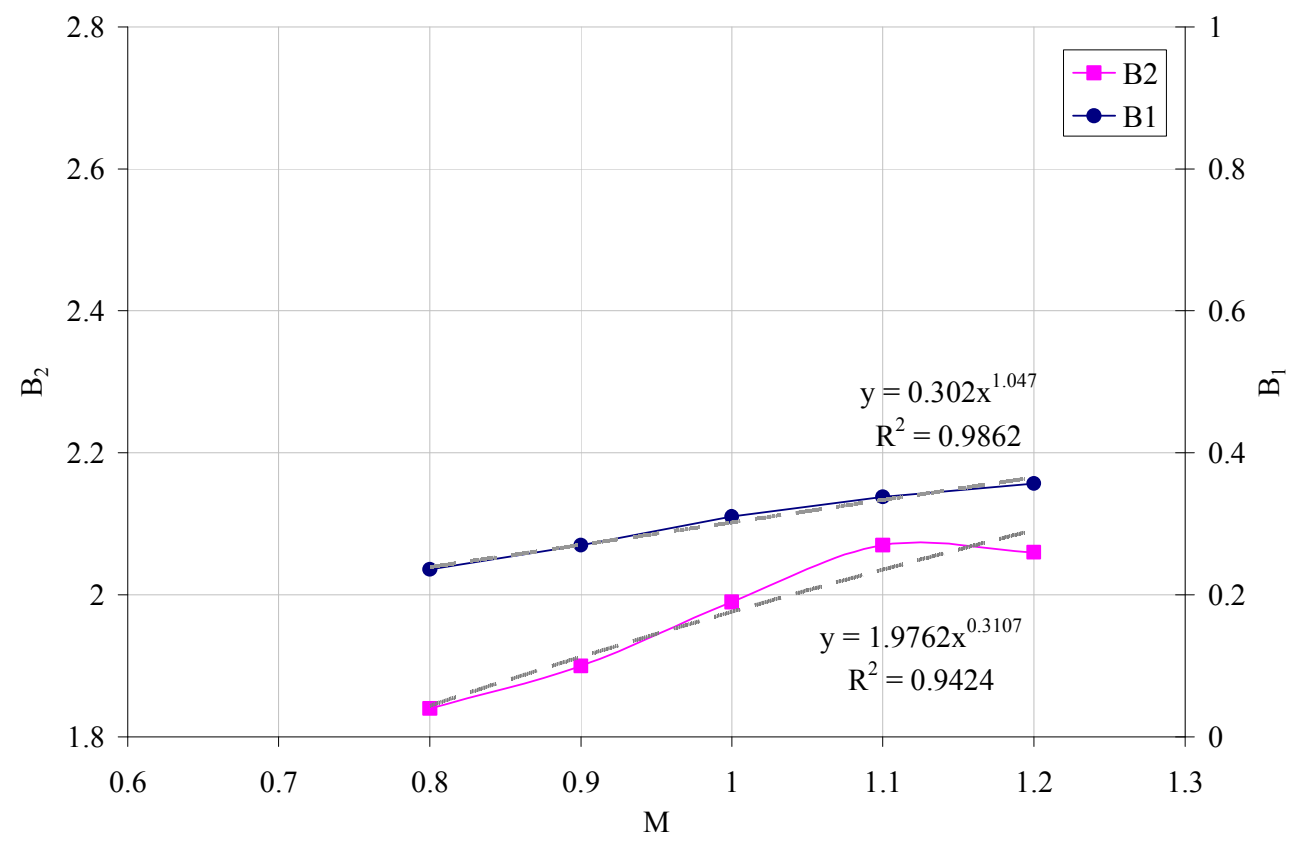

Figure 4-49 Variation of $B_{1}$ and $B_{2}$ with $M$ 


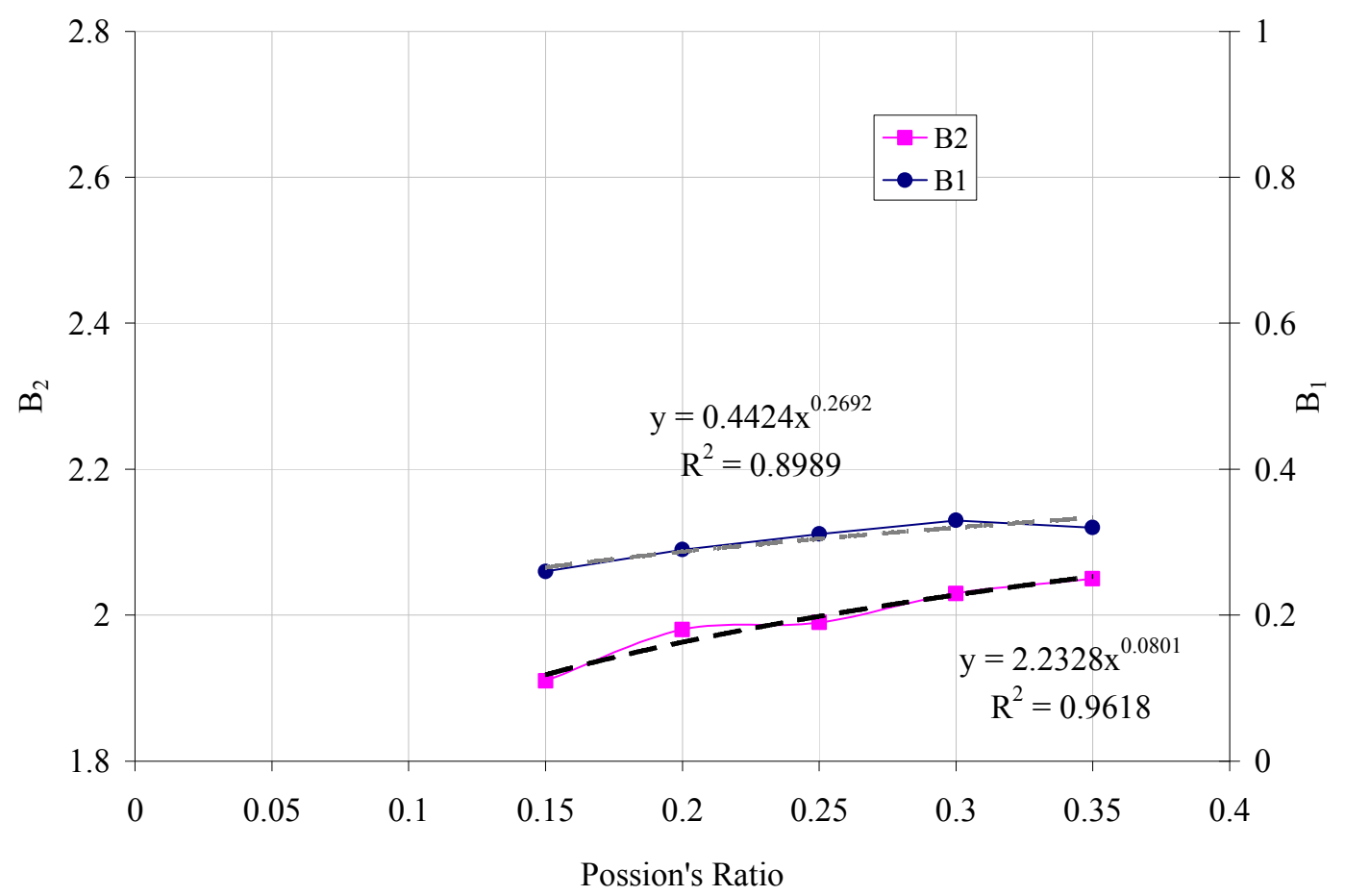

Figure 4-50 Variation of $\mathrm{B}_{1}$ and $\mathrm{B}_{2}$ with Poisson's ratio

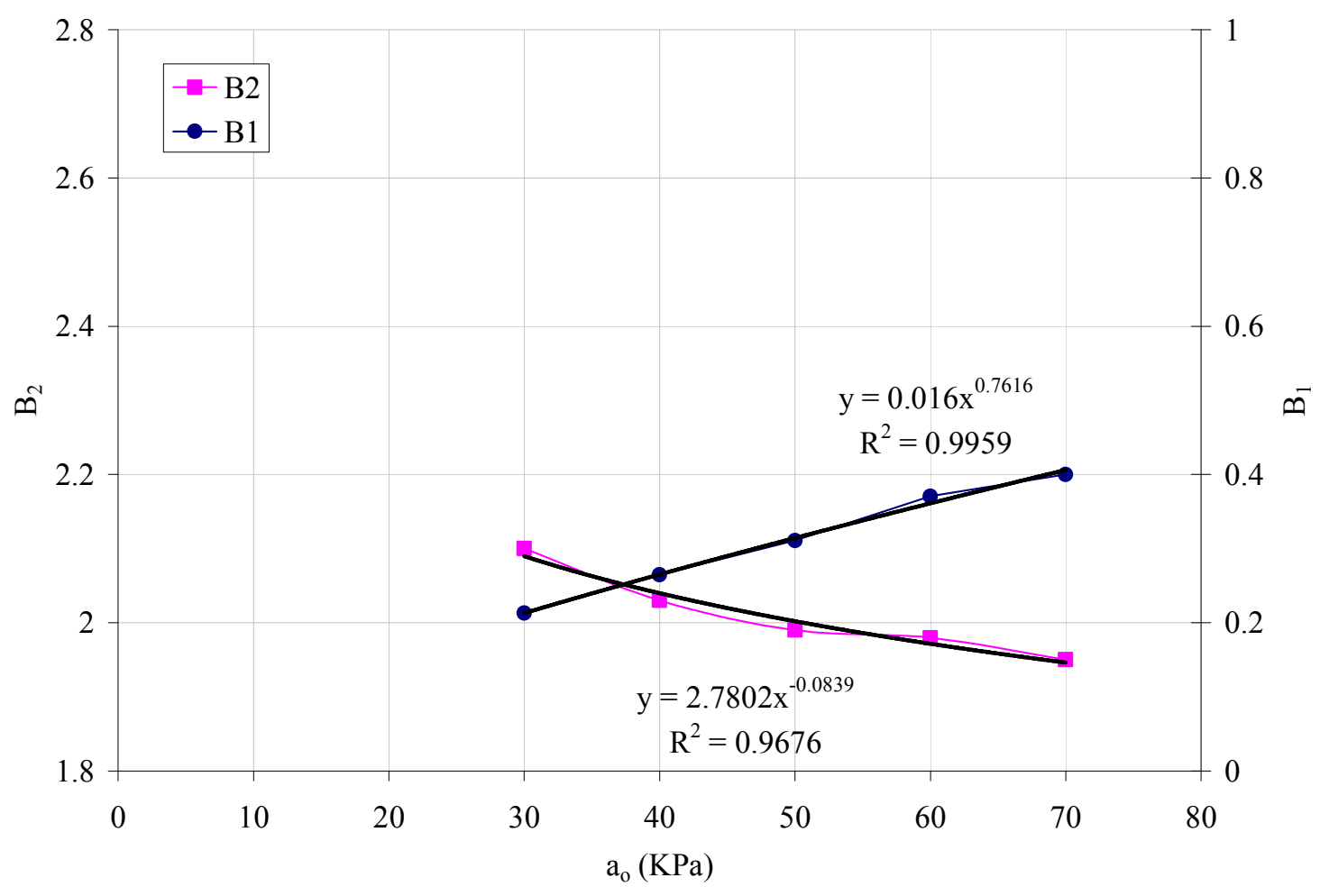

Figure 4-51 Variation of $\mathrm{B}_{1}$ and $\mathrm{B}_{2}$ with $\mathrm{a}_{\mathrm{o}}$ 


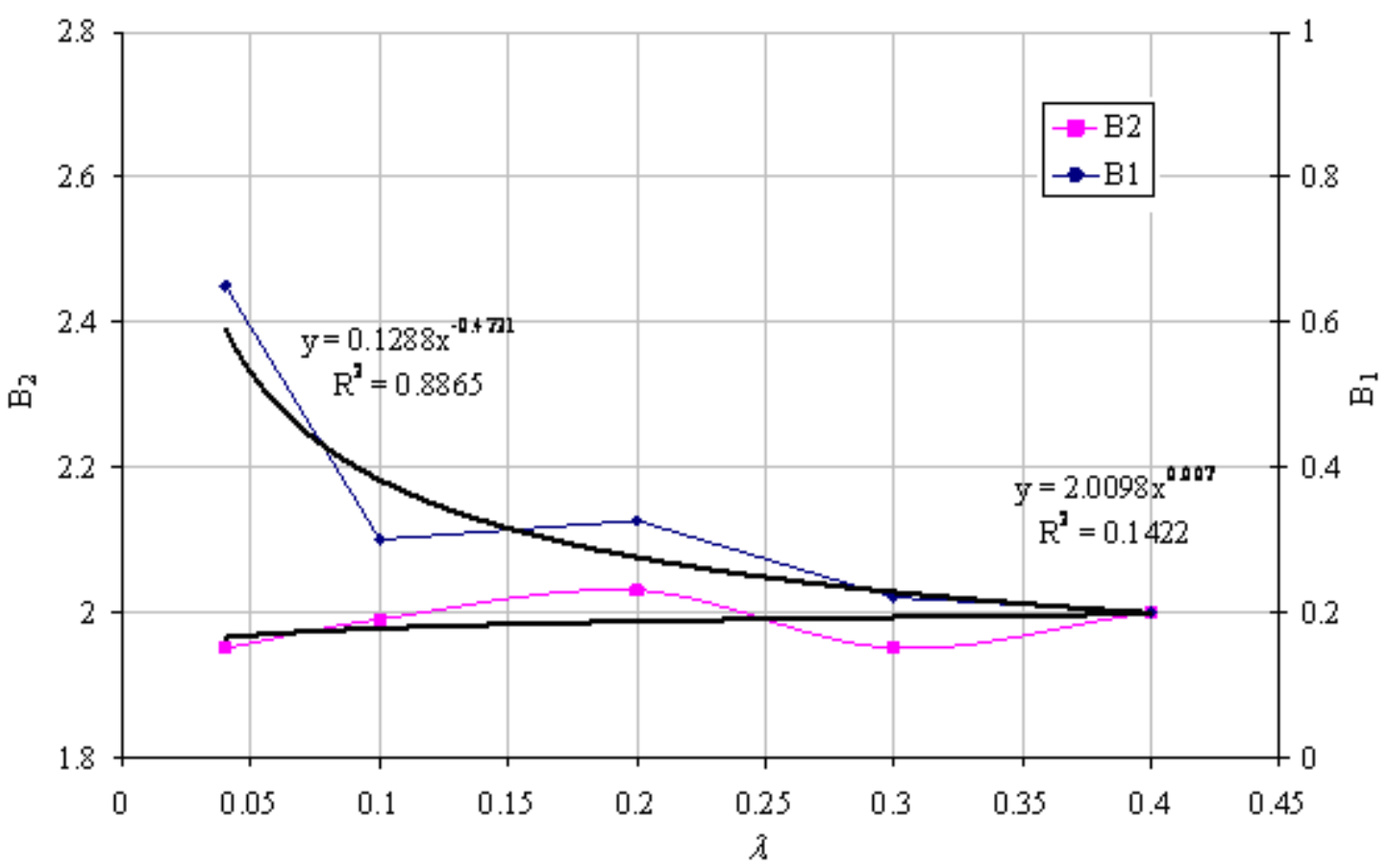

Figure 4-52 Variation of $\mathrm{B}_{1}$ and $\mathrm{B}_{2}$ with $\lambda$

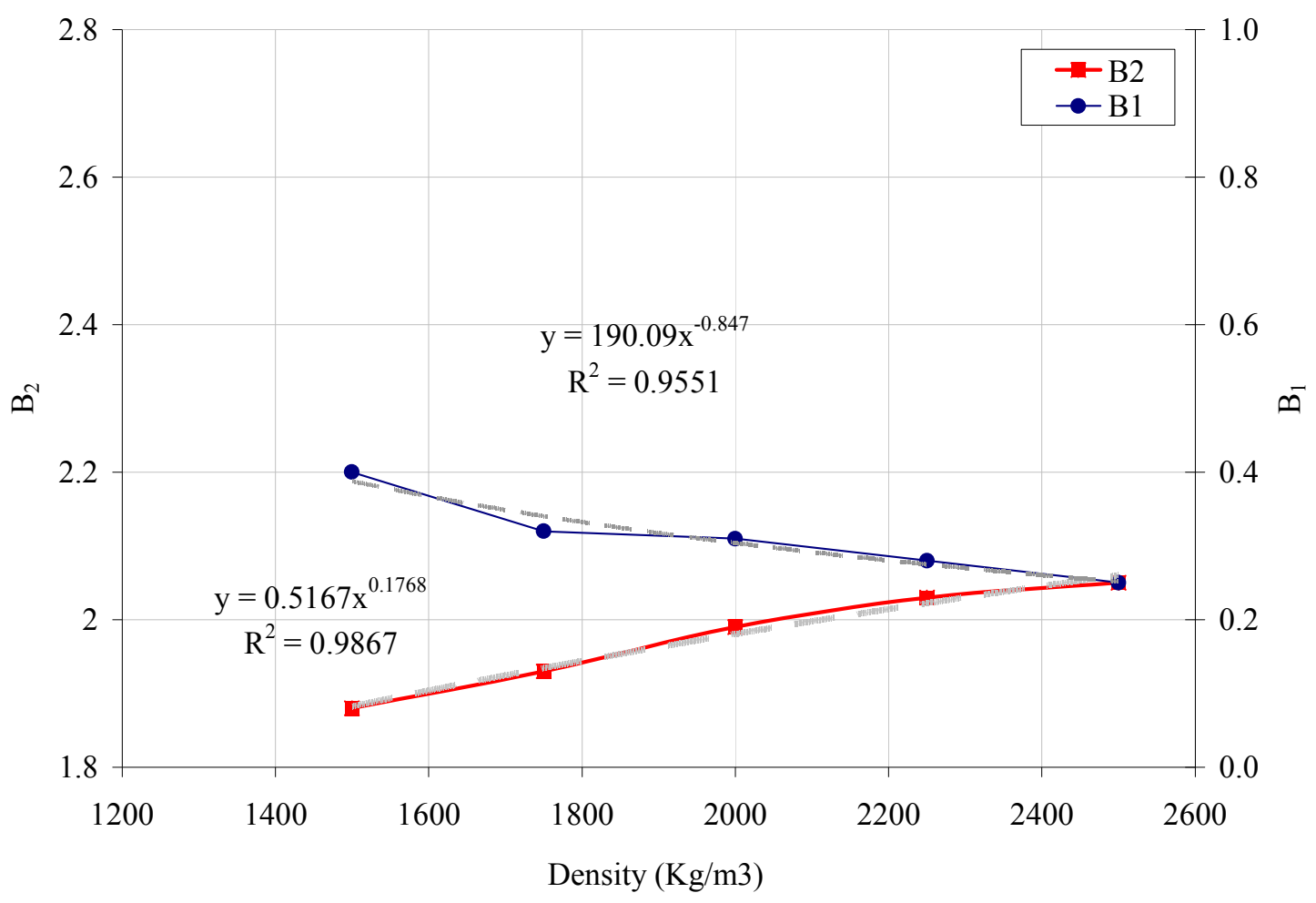

Figure 4-53 Variation of $\mathrm{B}_{1}$ and $\mathrm{B}_{2}$ with Density 
Table 4-6 Importance analysis for $\mathrm{B}_{1}$

\begin{tabular}{|l|c|c|c|c|c|c|}
\cline { 2 - 7 } \multicolumn{1}{c|}{} & $\rho$ & $\ddots$ & $M$ & $a_{o}$ & $e_{o}$ & $\lambda$ \\
\hline Max & 0.403 & 0.344 & 0.379 & 0.405 & 0.405 & 0.680 \\
\hline Min & 0.261 & 0.276 & 0.252 & 0.216 & 0.216 & 0.247 \\
\hline Max-Min & 0.142 & 0.068 & 0.126 & 0.188 & 0.188 & 0.433 \\
\hline Max-Min/ $\sum$ (Max- Min) (\%) & $12.38 \%$ & $5.94 \%$ & $11.01 \%$ & $16.45 \%$ & $16.45 \%$ & $37.78 \%$ \\
\hline
\end{tabular}

Table 4-7 Importance analysis for $\mathrm{B}_{2}$

\begin{tabular}{|l|c|c|c|c|c|c|}
\cline { 2 - 7 } \multicolumn{1}{c|}{} & $\rho$ & $v$ & $M$ & $a_{o}$ & $e_{o}$ & $\lambda$ \\
\hline Max & 2.07 & 0.34 & 2.04 & 2.10 & 2.03 & 2.00 \\
\hline Min & 1.88 & 0.28 & 1.90 & 1.85 & 1.96 & 1.93 \\
\hline Max-Min & 0.18 & 0.07 & 0.13 & 0.26 & 0.07 & 0.07 \\
\hline Max-Min/ $\sum$ (Max-Min) (\%) & $23.45 \%$ & $8.80 \%$ & $17.26 \%$ & $33.07 \%$ & $8.72 \%$ & $8.70 \%$ \\
\hline
\end{tabular}

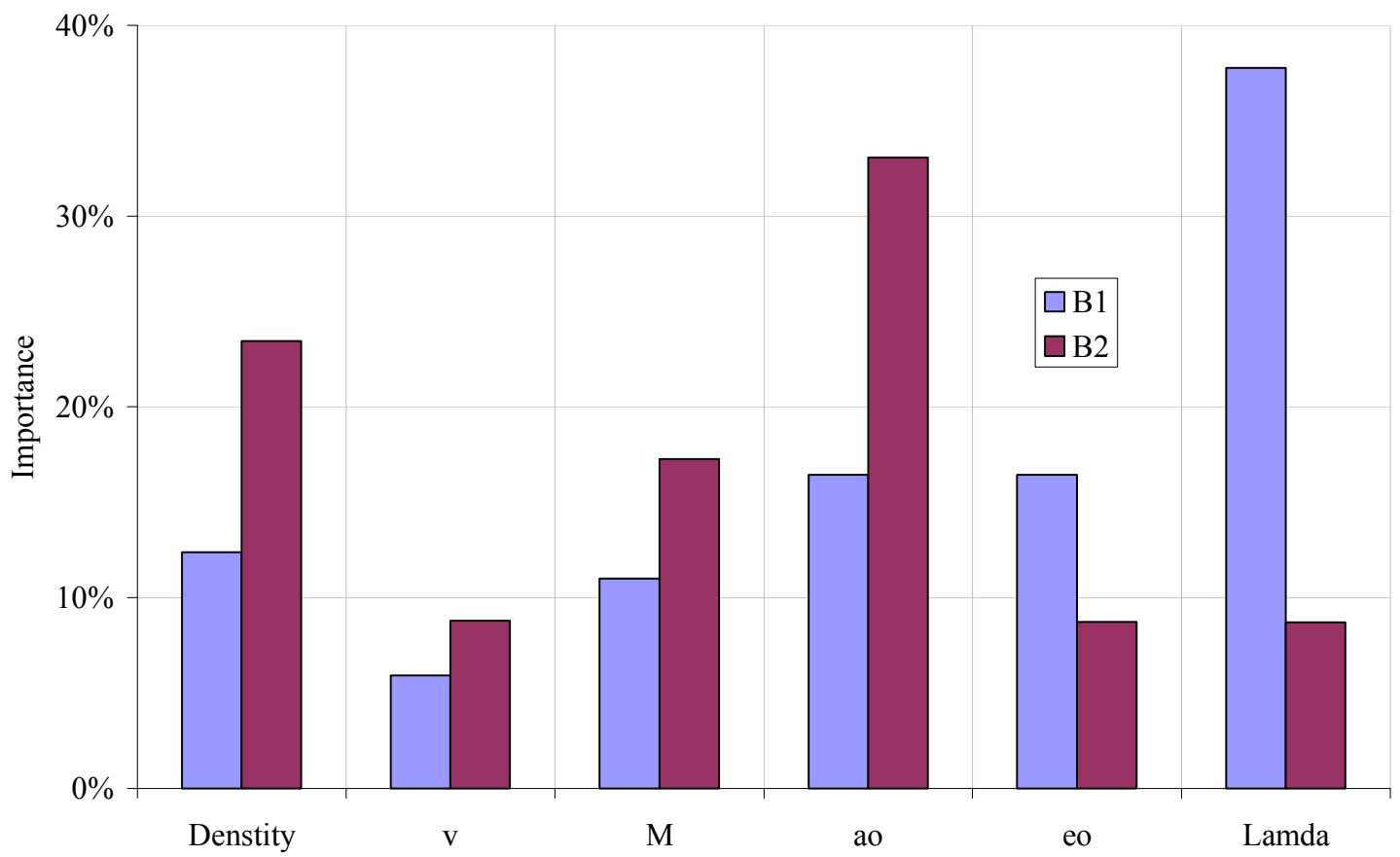

Figure 4-54 Importance analysis For $\mathrm{B}_{1}$ and $\mathrm{B}_{2}$ 


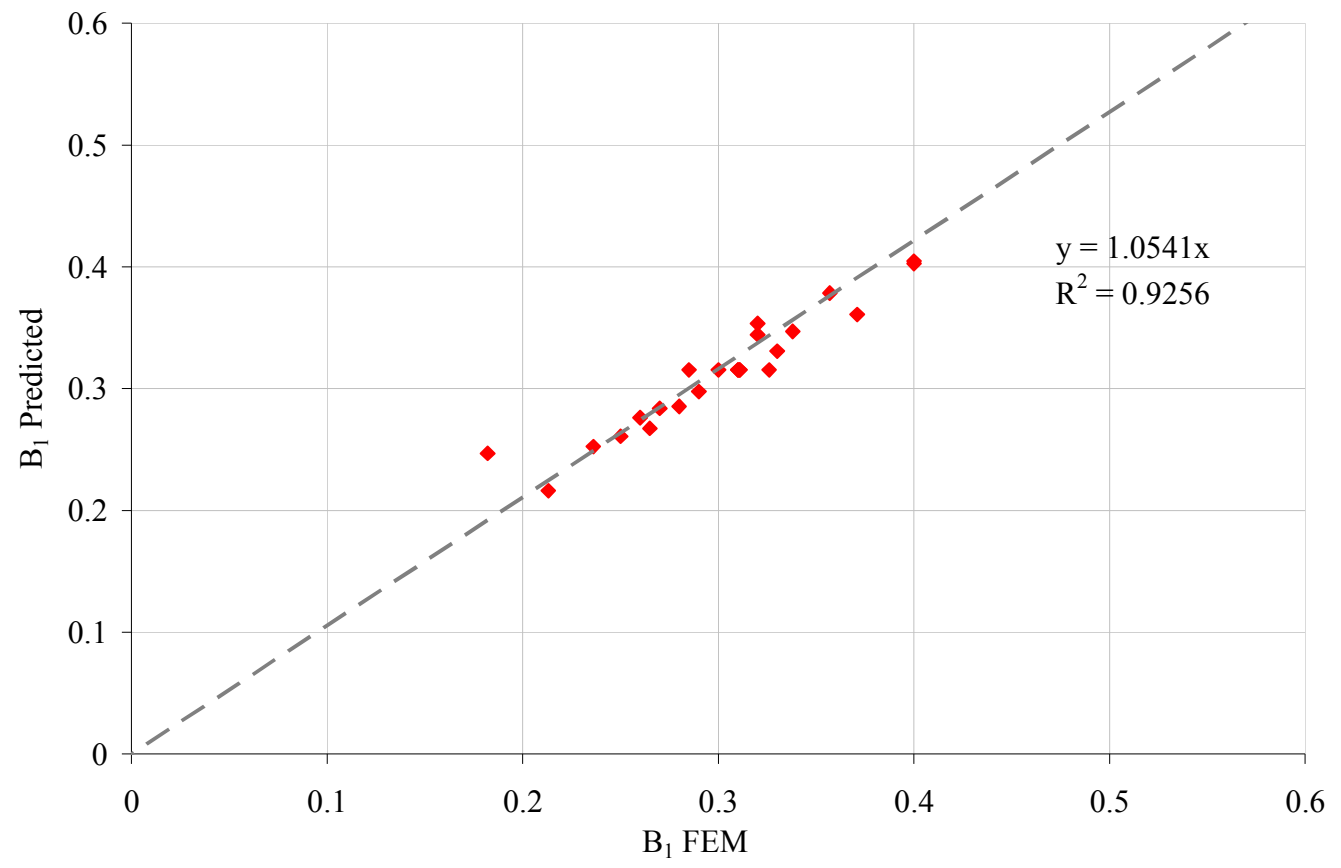

Figure 4-55 Model Validation

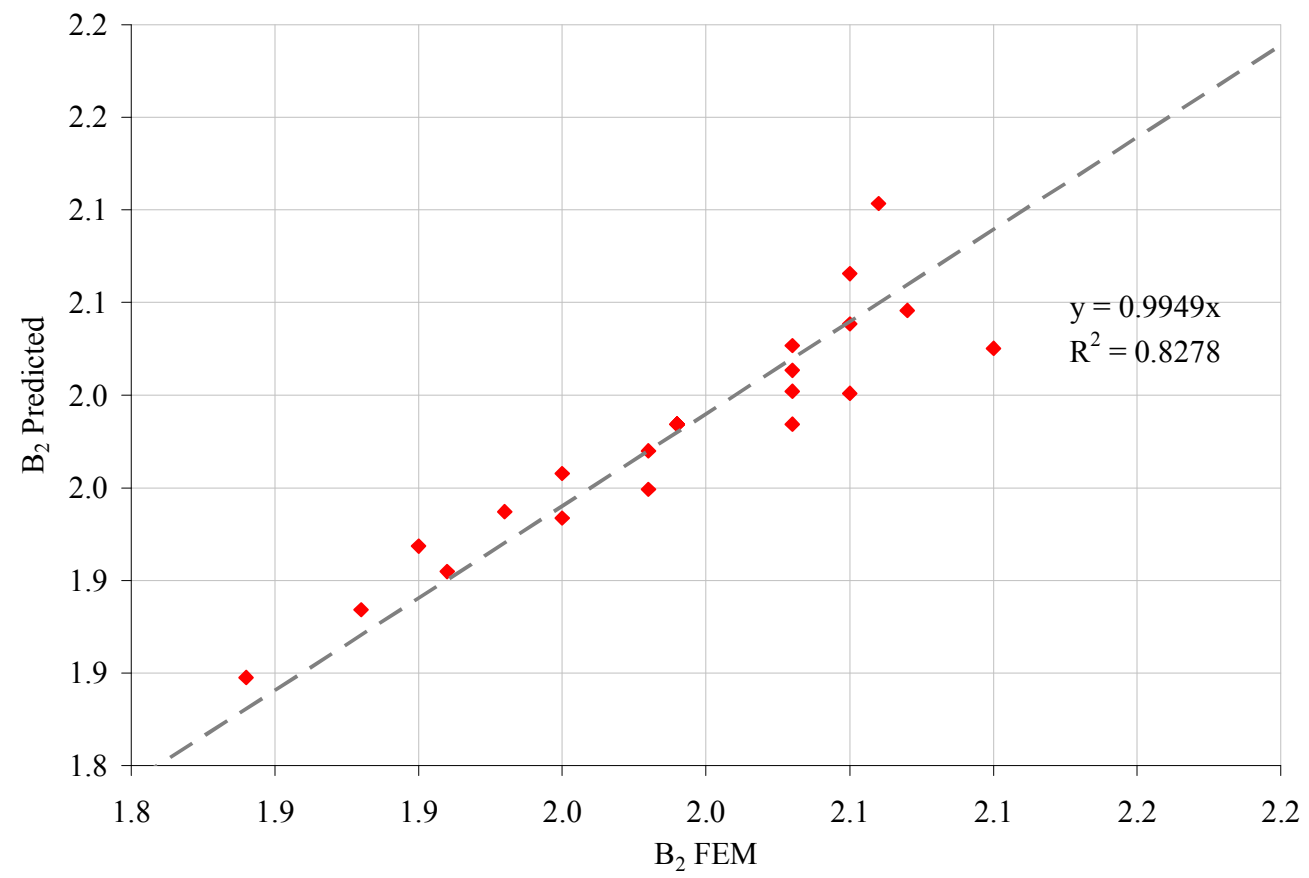

Figure 4-56 Model Validation 


\subsubsection{Frequencies Content of The Associated Waves}

In addition to the magnitude of Peak Particle Velocity (PPV), the frequency content of the waves generated by DC was investigated. The frequency range of the those waves is usually below $12 \mathrm{~Hz}$. Menrad (1975) reported, based on field measurements, that the most noticed frequencies fall between 3-4 Hz.

The frequency content of the associated waves can be investigated by studying the frequency content of the deceleration time function of the tamper mass. Figure 4-57 show the deceleration time function of the tamper mass. The deceleration time function was analyzed using Fast Fourier Transform (FFT) built-in function in MATLAB. The frequency content is shown in Figure 4-58. It can be seen that the frequencies associated with DC in cohesive soils are below $15 \mathrm{~Hz}$, which matches the field measurements presented by Menrad (1975)

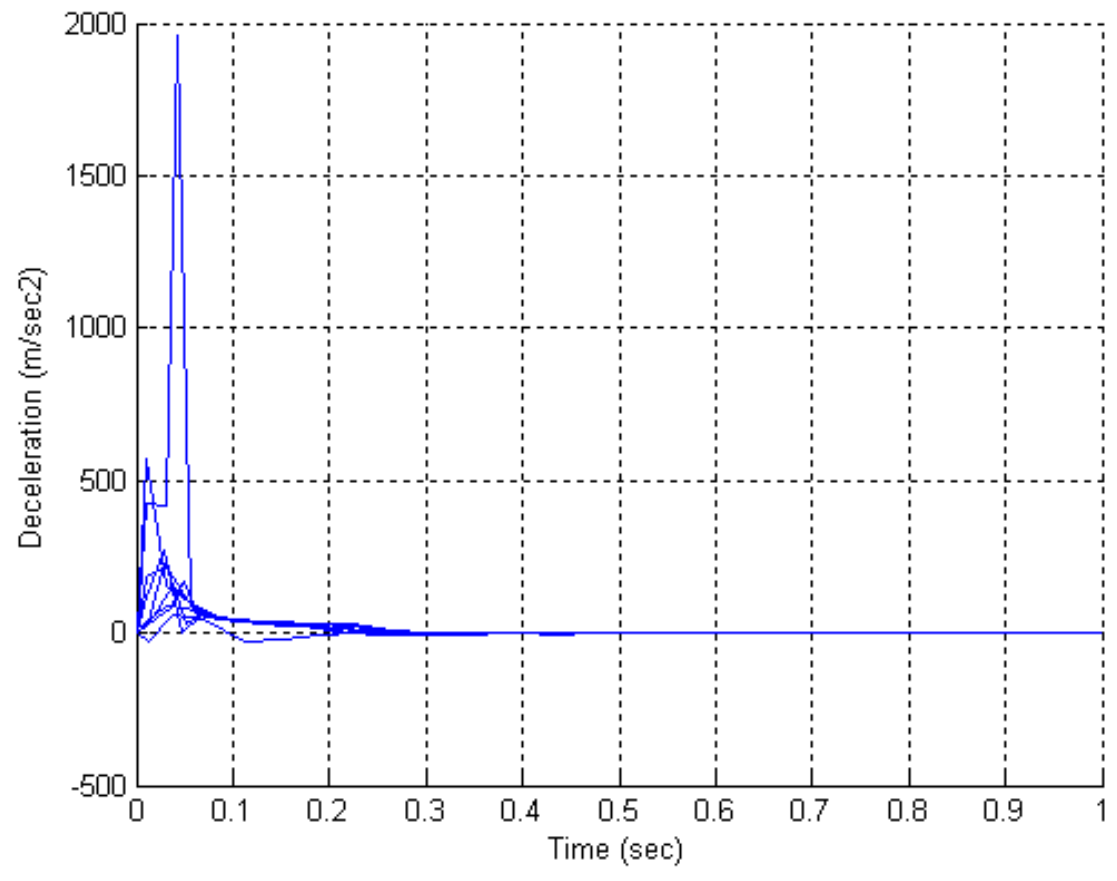

Figure 4-57 Tamper acceleration 


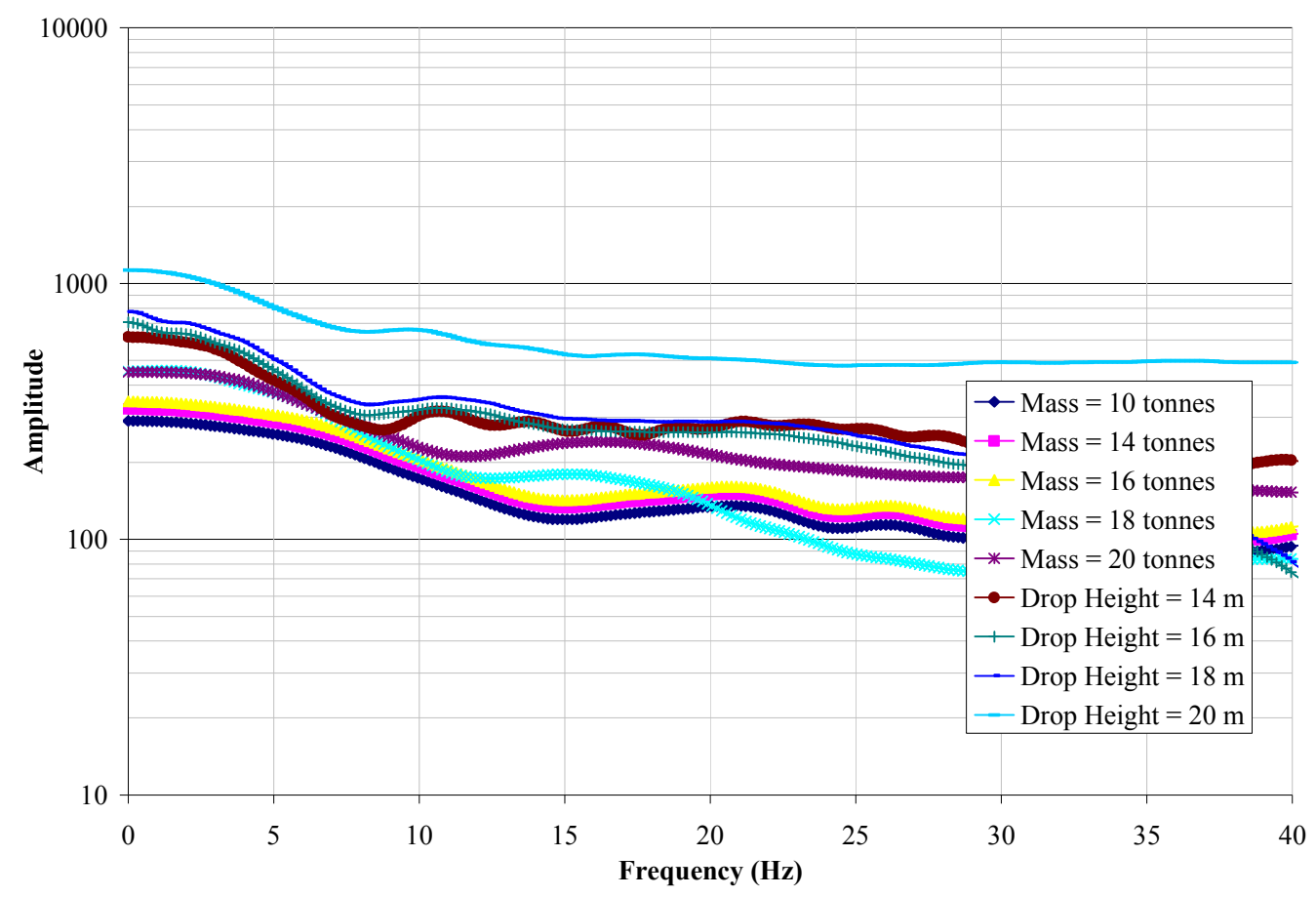

Figure 4-58 Frequency content of the tamper acceleration

\subsection{Summary and Conclusions}

In this chapter, a parametric study using FE model developed and validated in chapter III was conducted. Based on literature review, a range of Cam-Clay constitutive model parameters was identified and used to perform the parametric study. Sensitivity and important analysis was conducted to investigate the influence of tamping energy per blow on the crater depth $(\mathrm{P})$, void ratio $\left(\Delta e_{0}\right)$ distribution and, the Peak Particle Velocity (PPV). Soil properties were found to have significant influence on the estimation of crater depth $(\mathrm{P})$, void ratio $\left(\Delta e_{0}\right)$ distribution, and the Peak Particle Velocity (PPV).

For each model, the influence of tamping energy or momentum was investigated using the base line model results in which soil properties were kept constant at the base line value while changing the tamping energy. At the end of this step, a correlation was 
proposed. Then, more thorough investigation was performed to check the influence of Cam-Clay constitutive model parameters on the proposed correlation constants.

A Correlation equation to estimate the crater depth was developed. The crater depth could be related to the normalized tamper momentum. A parameter $\mathrm{C}$ was used as the proportionality constant. Further investigation on the variation of the parameter $\mathrm{C}$ with soil properties showed that the parameter $\mathrm{C}$ is proportional to the logarithmic plasticity bulk modulus as well as the initial void ratio. Soil density before compaction, slope of CSL line (M) and the pre-consolidation pressure were found to be inversely proportional to the crater depth.

A correlation equation was proposed for estimating the void ratio distribution as function of the normalized tamping energy. Two parameters $\mathrm{C}_{1}$ and $\mathrm{C}_{2}$ were used for the correlations. The sensitivity analysis showed that soil parameters have significant influence on $\mathrm{C}_{1}$ and $\mathrm{C}_{2}$.

Additional correlation equation was developed to estimate the PPV as function of tamping energy, distance from the tamping center and soil properties. Two parameters $\mathrm{B}_{1}$ and $\mathrm{B}_{2}$ were used for the correlations. The sensitivity analysis showed that soil parameters have significant influence on $\mathrm{B}_{1}$ and $\mathrm{B}_{2}$.

The following can be concluded:

FE was successful in modeling the DC operation is cohesive soils using Cam-Clay constitutive model

Crater depth (P), void ratio distribution $\left(\Delta e_{0}\right)$, and Peak Particle Velocity (PPV) are sensitive to soil properties as well as tamping energy.

Statistically reliable design model was developed for DC in cohesive soils. 


\section{CHAPTER V}

\section{EFFECT OF MULTIPLE IMPACTS FROM ADJACENT IMPACT SPOTS}

\subsection{Introduction}

In this chapter, the finite element code ABAQUS was used to develop a threedimensional model for the dynamic compaction in cohesive soils described by a CamClay constitutive model. The objective of this study was to achieve a better understanding about the interaction between the adjacent drops of an impact mass. The 3-D model consists of four tamping locations (spots), with each spot being tamped four times before moving to the next spot. The footprint center to center spacing was modeled as 2.5 and 3.0 times of the tamper radius. Interaction between the nearby tamping was evaluated at the center point of the footprint as well as the middle point of the footprint side. The selection of these two points for study was based on a literature review, which indicates that they are the locations with the least improvement. Void ratio distribution along the depth at the mentioned points were evaluated and compared to the void ratio distribution

directly beneath the tamper. Based on the analysis presented, suitable footprint spacing was recommended for DC operation in cohesive soil. The work presented in this chapter contributes to enhancing existing knowledge of dynamic compaction through a detailed 3D FE simulation study. 


\subsection{Background}

Dynamic Compaction (DC) is a soil improvement technique in which soil is tamped heavily using a large mass dropped from a pre-determined height to densify soil underneath the tamper. DC has been used successfully in improving both cohesive and cohesionless soil deposits. The technique is known to be economic, easy to implement, and environmentally safe. Empirical or semi-empirical models were presented in the literature to estimate the depth of improvement and the degree of improvement as a function of tamping energy.

The current empirical models were based on wealthy field measurements. For example, an empirical expression for the depth of improvement was formulated as $D I=n \sqrt{W H}$, where $\mathrm{n}$ is a multiplier depending on soil type, $\mathrm{W}$ is tamper mass in tons, $\mathrm{H}$ is the drop height in meter. Menrad and Broise (1975) estimated the value of multiplier $\mathrm{n}$ to equal 0.5. Mitchel (1981) and Mayne (1984) summarized field measurements for considerable number of sites and generated charts representing energy of tamper impact versus depth of influences based on which empirical expressions to estimate depth of influence and peak particle velocity (PPV) of the associated waves could be obtained. Mayne et al (1981) concluded that the multiplier $\mathrm{n}$ falls between 0.3-0.8 depending on soil properties.

In a study to investigate the lateral extent of treatment, Poran (1992) derived a correlation between the plastic zone dimension, which is assumed to have a semispheroid shape, and the tamping energy. The study was conducted using small scale laboratory tests in which tamping took place over one spot. The study was conducted 
using a tamper diameter ranges from 15 to $23(\mathrm{~cm})$ and the drop height ranges from 1 to 2 meters. The total energy per below ranged from 440 to $660 \mathrm{~N}-\mathrm{m}$. The highest energy per unit area in Poran's work is $36,000\left(\mathrm{~N}-\mathrm{m} / \mathrm{m}^{2}\right)$. This is similar to a tamper with 10 tones mass and $1.25(\mathrm{~m})$ radius dropped from $\sim 18(\mathrm{~m})$.

Chow (1994) investigated the lateral extent of DC operation at the ground surface by conducting cone penetration test (CPT) for a series of DC operations in the field. Myerhof's correlation between the CPT results and friction angle was utilized. Correlation between the increase in soil friction angle and the distance measured from the center of tamping was developed. Chow also reported that, at distance three times the tamper diameter, the effect of the DC is negligible. Moreover, when compaction is placed over a spaced grid, the center point of the grid and the middle point of the grid sides are points of weakness as they receive the least compaction effects.

Some numerical simulations efforts were made to utilize finite element codes to study dynamic compaction in different types of soils. However, these studies were limited to the use of a simplified elastic perfectly plastic constitutive model. Furthermore, soil density after compaction was not studied. In addition, the effect of multiple impacts from adjacent impact spots has not been studies carefully yet.

The purpose of this chapter is to utilize ABAQUS FE code to study numerically the effect of multiple impacts from adjacent impact spots on the degree of soil improvement after compaction. The ABAQUS built-in Cam-Clay constitutive model was used to represent the stress- strain behavior of the cohesive soils. Based on the FE simulation results, suitable footprint spacing was recommended for DC operation in cohesive soils. 


\subsection{3-D FE Modeling}

Figure 5-1 shows the 3-D FE model. The soil body was modeled as a cylinder of 7 meters in radius and 10 meters in height. The radius of the cylinder was selected after several iterations to avoid interference from the constrained boundary condition on the dimensions of zone of influence. Similarly, the height of the model was selected to avoid any interference from the fixed bottom. 8-node brick node elements were used to model the soil continuum.

It is not possible to avoid the large deformations that occur during compaction, especially with multiple nearby drops case in which the crater depth ranges from 1 to 1.5 meters. Therefore, a simple technique was proposed to avoid severe element distortion. A triangle shaped groove with 0.2 meter width and 0.15 meter depth is placed around the peripheral of each tamping spot. Figure 5-2 shows the location and dimensions of the proposed groove. Several trials were conducted to assure negligible effect of the proposed groove on the development of crater depth and void ratio distribution underneath the tamper resulting from a single drop (10 tons dropped from 10 meters).

The tamper was modeled as circular disc with 1.25 meter in radius. Tamper material (concrete or steel) is often stiffer than soil. Therefore, tamper was modeled using rigid elements. Also, the tamper was constrained in all degrees of freedom (translational and rotational) except the vertical one. A force-time function was applied to the surface of the rigid element pushing it to strike on soil surface. 


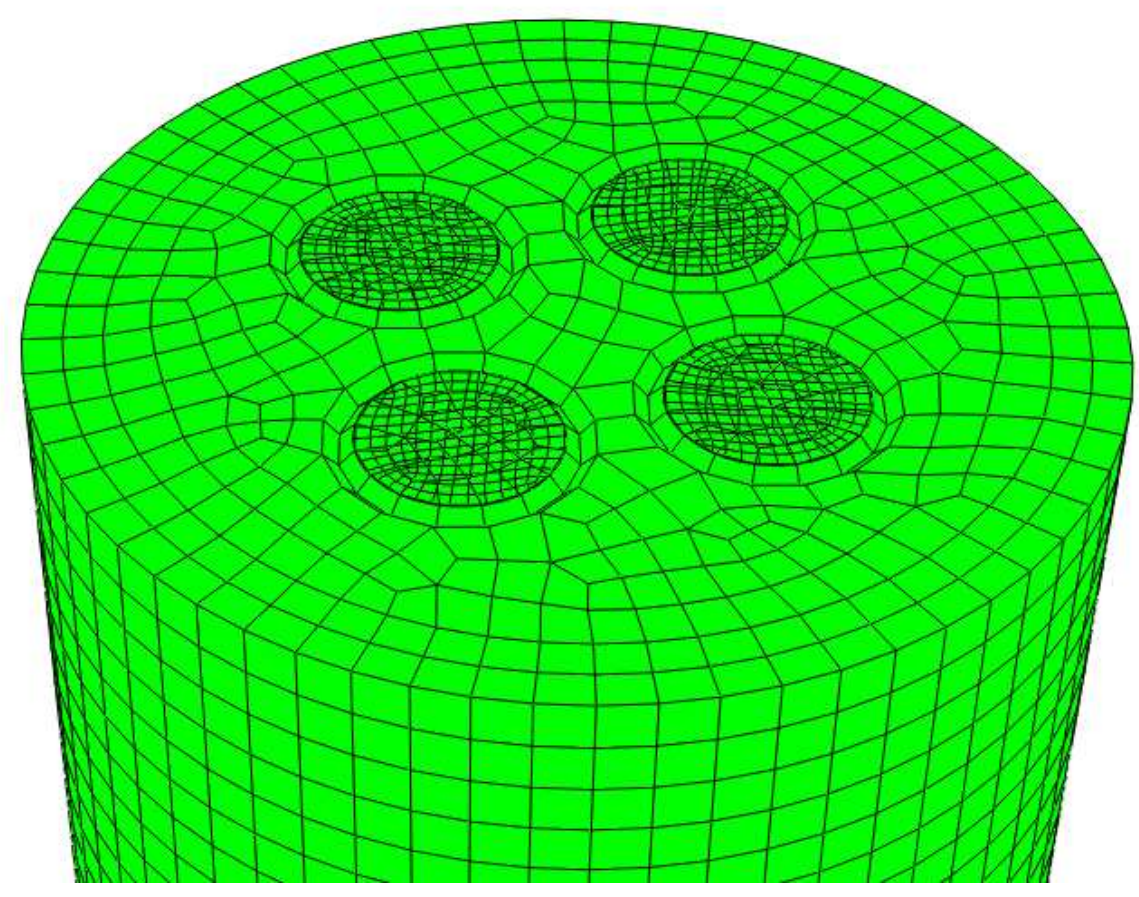

Figure 5-1 FE mesh for dynamic compaction modeling

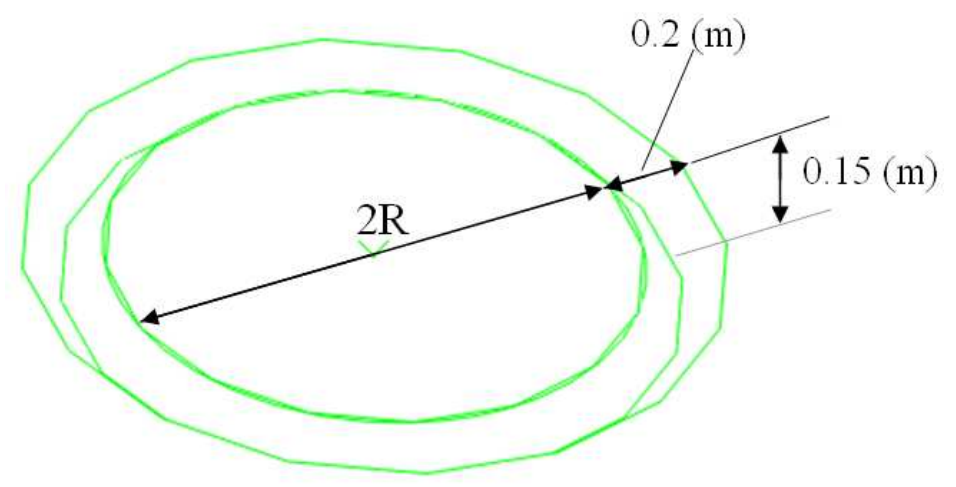

Figure 5-2 Proposed groove

Cam-Clay material constitutive model was used to represent the cohesive soils in the model. The adoption of the Cam-Clay model is based upon its ability to represent soil stress-strain behavior more realistically than other simplified models (Von -Misses or Mohr-Coulomb). In addition, softening and hardening behavior can be captured by the 
model. There are a few Cam-Clay model parameters that need to be obtained by using tri-axial laboratory test results. A survey of the typical Cam-Clay model parameters from the existing in the literature is presented in Table 5-1.

Table 5-1 Cam-Clay model parameters for the 3-D model

\begin{tabular}{l|c|c}
\hline Parameter & Symbol & Value \\
\hline Soil density & $\rho(\mathrm{kg} / \mathrm{m} 3)$ & 2000 \\
\hline Poisson's ratio & $v$ & 0.25 \\
\hline Logarithmic plastic bulk modulus & $\lambda$ & 0.20 \\
\hline Logarithmic elastic bulk modulus & $\kappa$ & 0.02 \\
\hline Initial yield surface & $a_{0}(\mathrm{kPa})$ & 50 \\
\hline Critical state line slope & $M$ & 1.00 \\
\hline Initial void ratio & $e_{0}$ & 1.0 \\
\hline
\end{tabular}

Geostatic analysis is a built in step (analysis) in ABAQUS FE code for modeling gravity induced stresses. The state of equilibrium between the applied gravity load and soil stresses should be reached while resetting the deformation field at zero. The initial conditions are defined by calculating the vertical and horizontal stresses using specific soil weight and coefficient of lateral earth pressure at rest. The coefficient of lateral earth pressure at rest is a function of soil friction angle which is related to the critical state line slope. Initial void ratio distribution is also defined during geostatic step analysis and assumed constant along the model depth.

The force-time function of tamper was used for modeling multiple drops, as shown in Figure 5-3. The method of determining appropriate force-time function will be discussed later in this chapter. A total of sixteen analysis steps (dynamic/implicit) were used to model the multiple drops. At each step, the prescribed force-time was applied. Each tamping spot was tamped four times before moving to the next spot. The model was 
developed to simulate a real life dynamic compaction operation. The model simulation for each step lasts for 0.25 second, which is approximately enough for the surface waves to reach the boundaries.

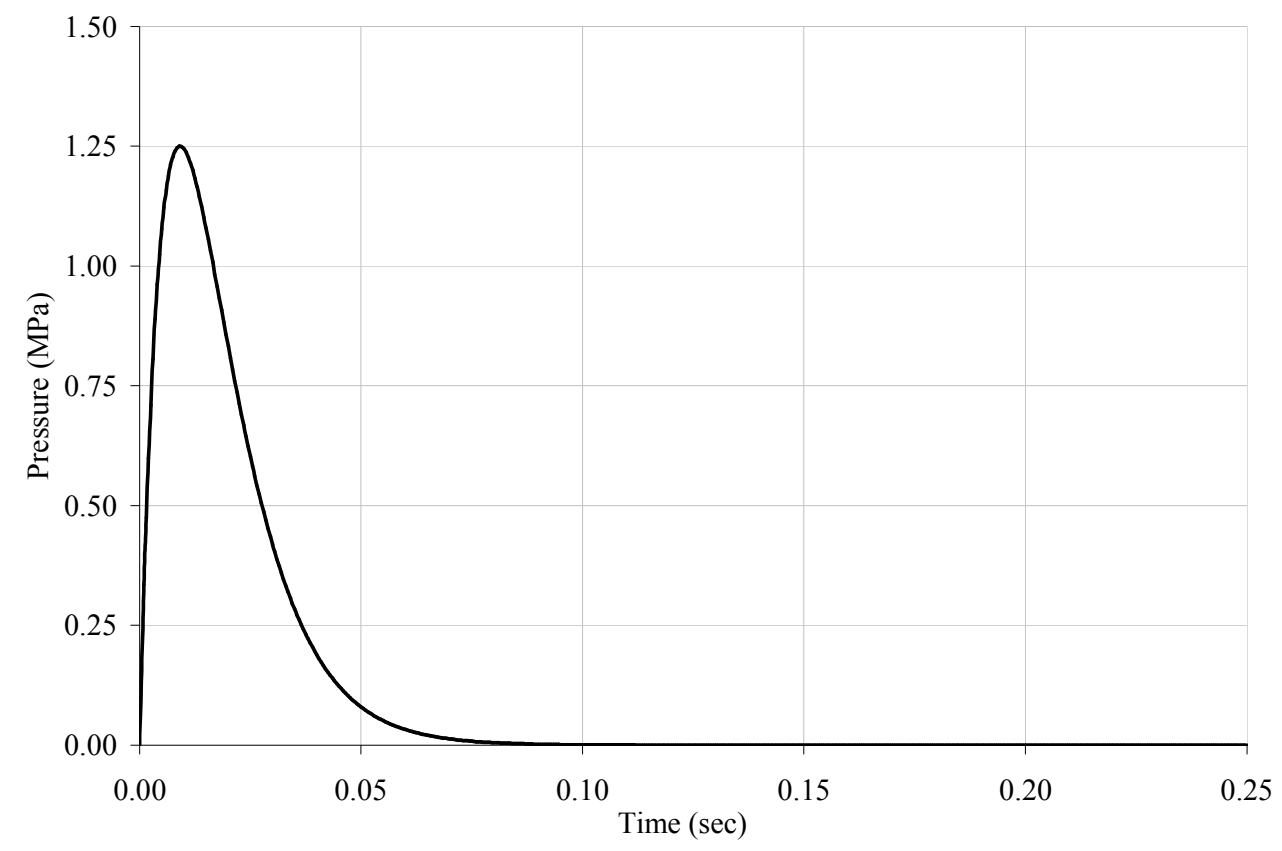

Figure 5-3 Transient pressure load

Element size was selected based on the review presented in section (3-2). According to Vailliappan (1984), 0.725 (m) was enough to model FE. several trails were conducted to check for mesh convergence, which resulted in selecting 0.4 meter element size as appropriate for the present study of DC The corresponding number of 8-node brick element is 22,000 elements.

Modeling of tamping in ABAQUS FE code can be done in two ways. The first is to assign initial velocity to the rigid tamper. The mass of the tamper is modeled as a concentrated mass at the center of the tamper. However, this technique cannot be used for multiple drops modeling since the initial can be assigned initial velocity just once. 
Therefore, an alternative technique was developed in the literature to model multiple drops. The alternative modeling technique replaces the initial velocity approach model by a force-time function as shown in Figure 5-3. The amplitude of the function is the one that produces the same effect as the one produced by the initial velocity model. It was found that applying force-time function with amplitude equal to $1.25 \times 106(\mathrm{~Pa})$ will have the same effect as striking the soil with a 10 ton mass dropped from 10 meter height. Figure 5-4 and Figure 5-5 compare ground response for the two modeling techniques (initial velocity and force-time function). Two criteria were used for the comparison; development of crater depth with time and the void ratio distribution after the first drop. It can be noticed that a good match can be achieved between the two modeling techniques. Therefore, the force time function can be used to model the multiple drops case. 


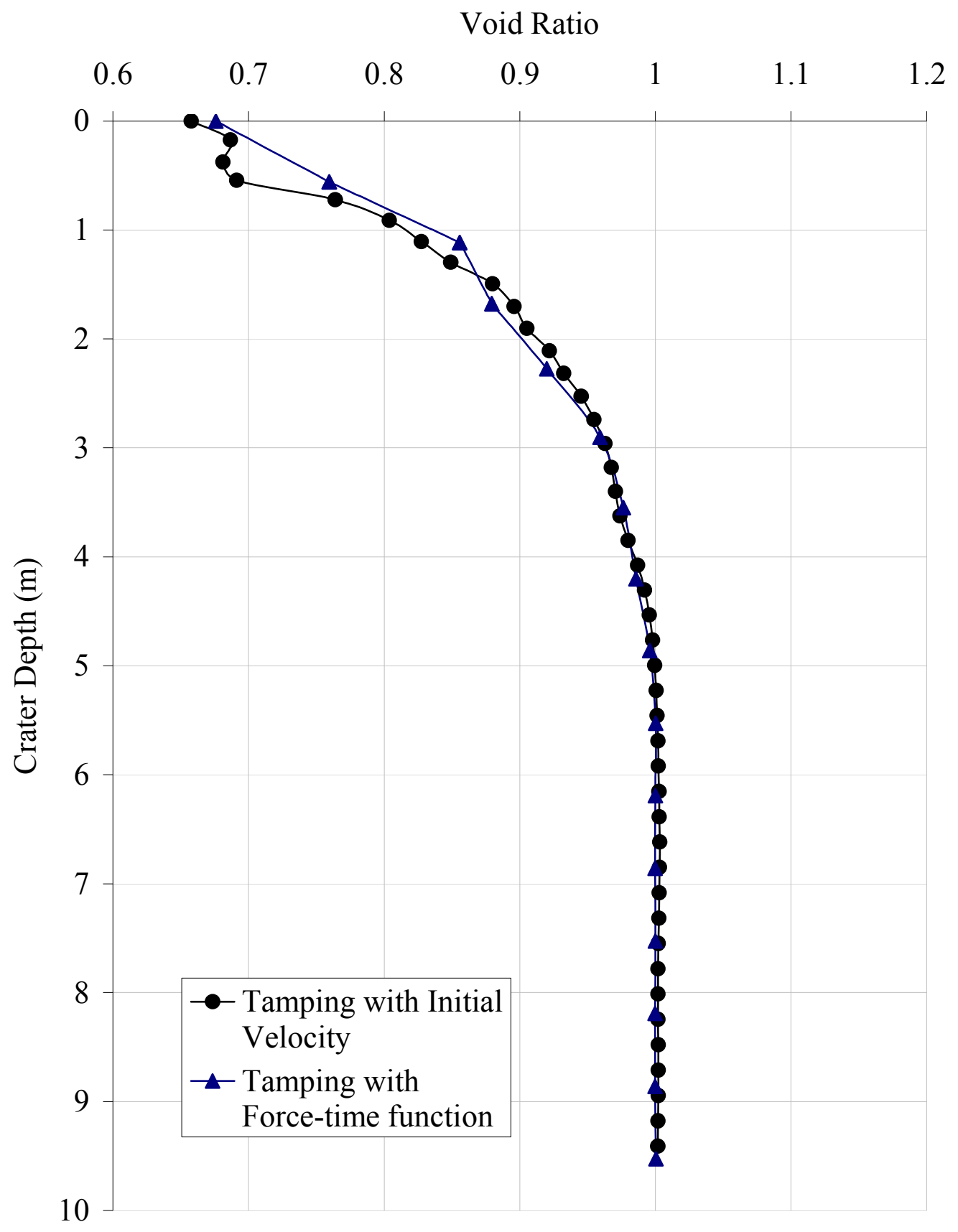

Figure 5-4 Comparison of void ratio distribution 


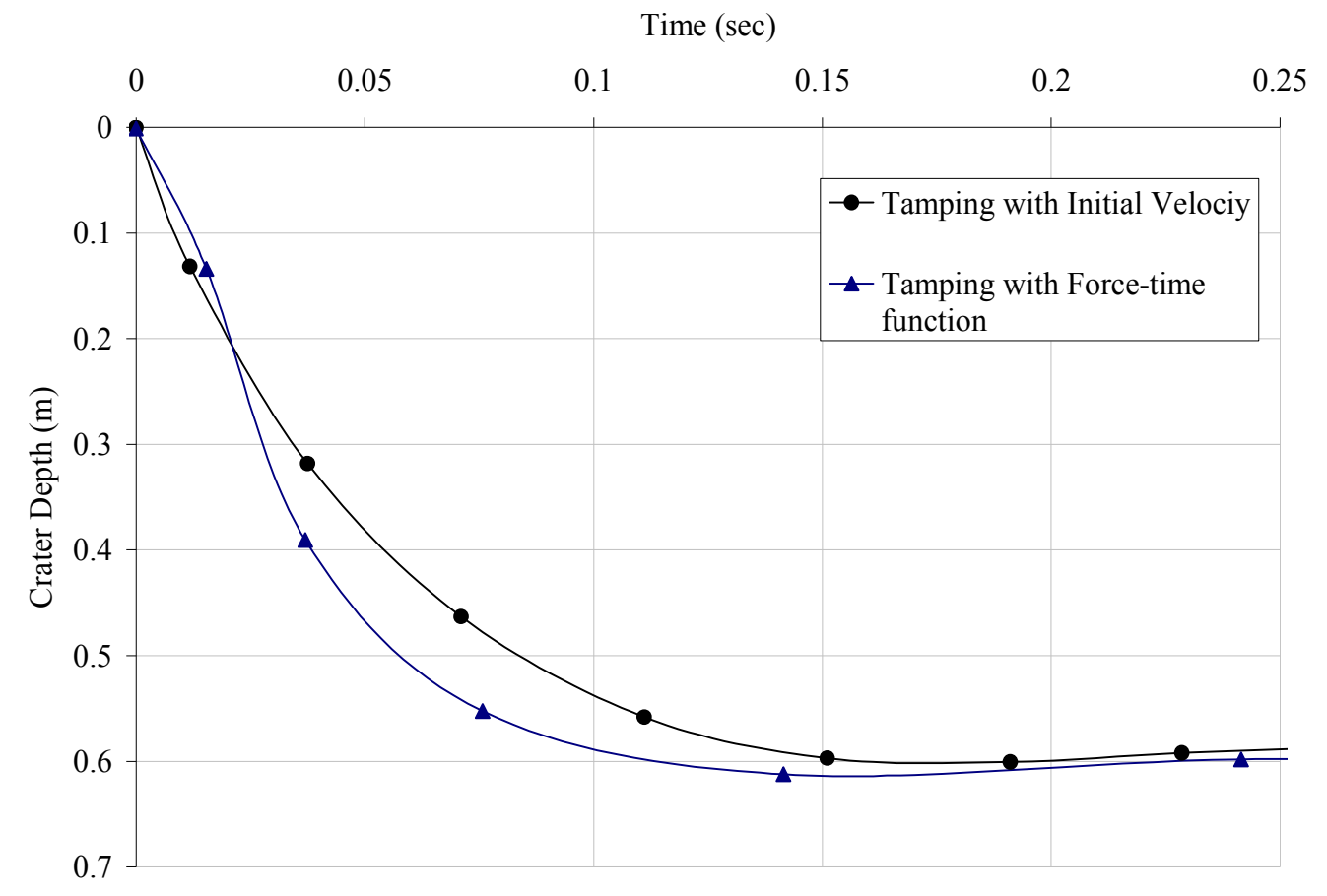

Figure 5-5 Comparison between crater depth

\subsection{Results and Discussions}

In this section, FE simulation results for two cases with different grid spacing are presented. The first case adopts a grid spacing of $3 \mathrm{R}$, where $\mathrm{R}$ is the tamper radius, and is equal to $3.9(\mathrm{~m})$. The second case adopts a grid spacing of $2.5 \mathrm{R}$ which is equal to $3.2(\mathrm{~m})$. Figure 5-6 shows the tamping sequence and the location of the evaluation points. Point A is located at the middle of footprint side, while Point B is located at the center of the footprint. The dashed lines on the schematic diagram are the lines of least treatment effects. Therefore, the subsequent evaluation of dynamic compaction focuses on points A and $\mathrm{B}$. 


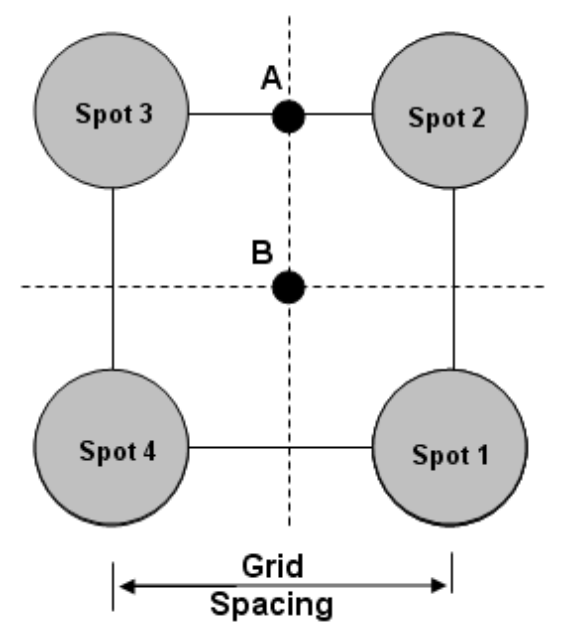

Figure 5-6 Schematic of footprint spacing

\subsubsection{Results for Model of 3R Footprint Spacing $(\mathrm{R}=1.25 \mathrm{~m})$}

As stated previously, a force time function is going to be used to model DC. The force-time function amplitude was selected to simulate tamping using 10 tons mass dropped from 10 meters height. Figure 5-7 shows the sequence of tamping over four spots. Each spot was tamped four times before moving to the other spots. The contour shown in Figure 5-7 is void ratio distribution. It can be noticed that the groove introduced in numerical simulations successfully prevented the formation of the heaves, which usually cause excessive distortion to the elements around the tamper. Figure 5-8 shows cross sections at depth 1, 2, 3 and 5 meters measured from the bottom of the crater. The interaction between the nearby tamping spots can be clearly noticed. The center of the grid receives the least treatment along the depth. The treatment at the footprint side midpoint is strongly influence by the interaction between the adjacent tamping drops. 


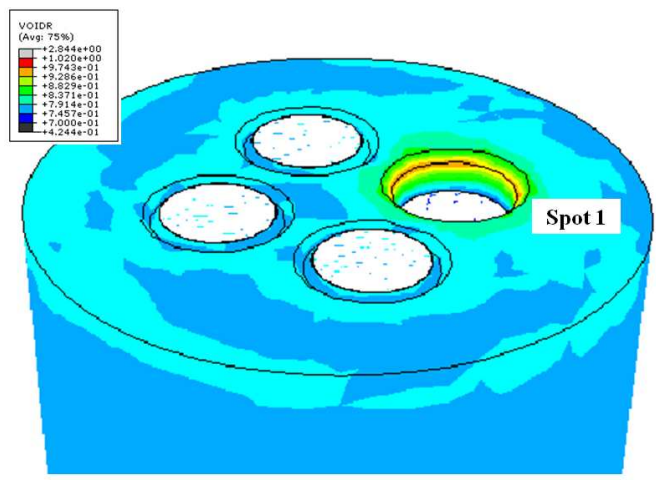

Tamping at spot 1

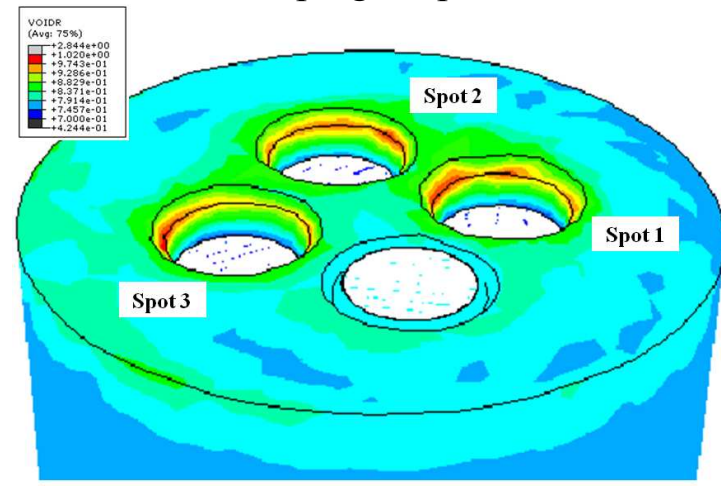

Tamping at spot 3

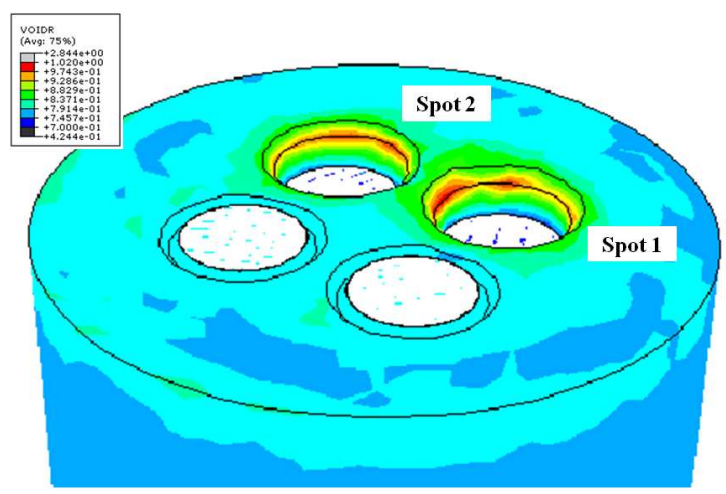

Tamping at spot 2

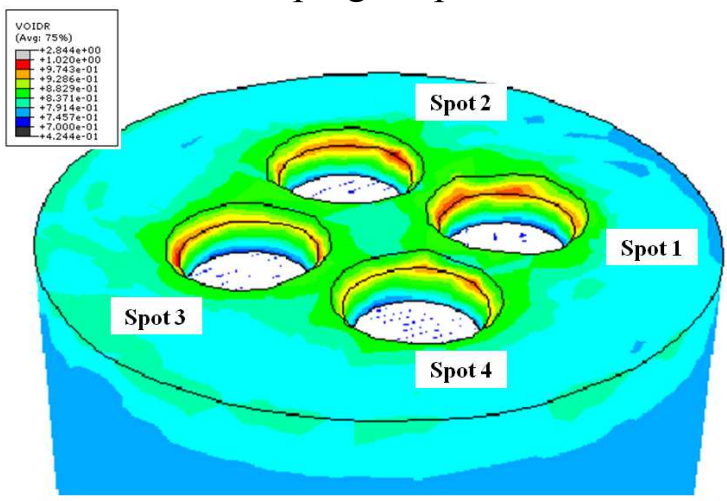

Tamping at spot 4

Figure 5-7 Tamping stages (3R model) 

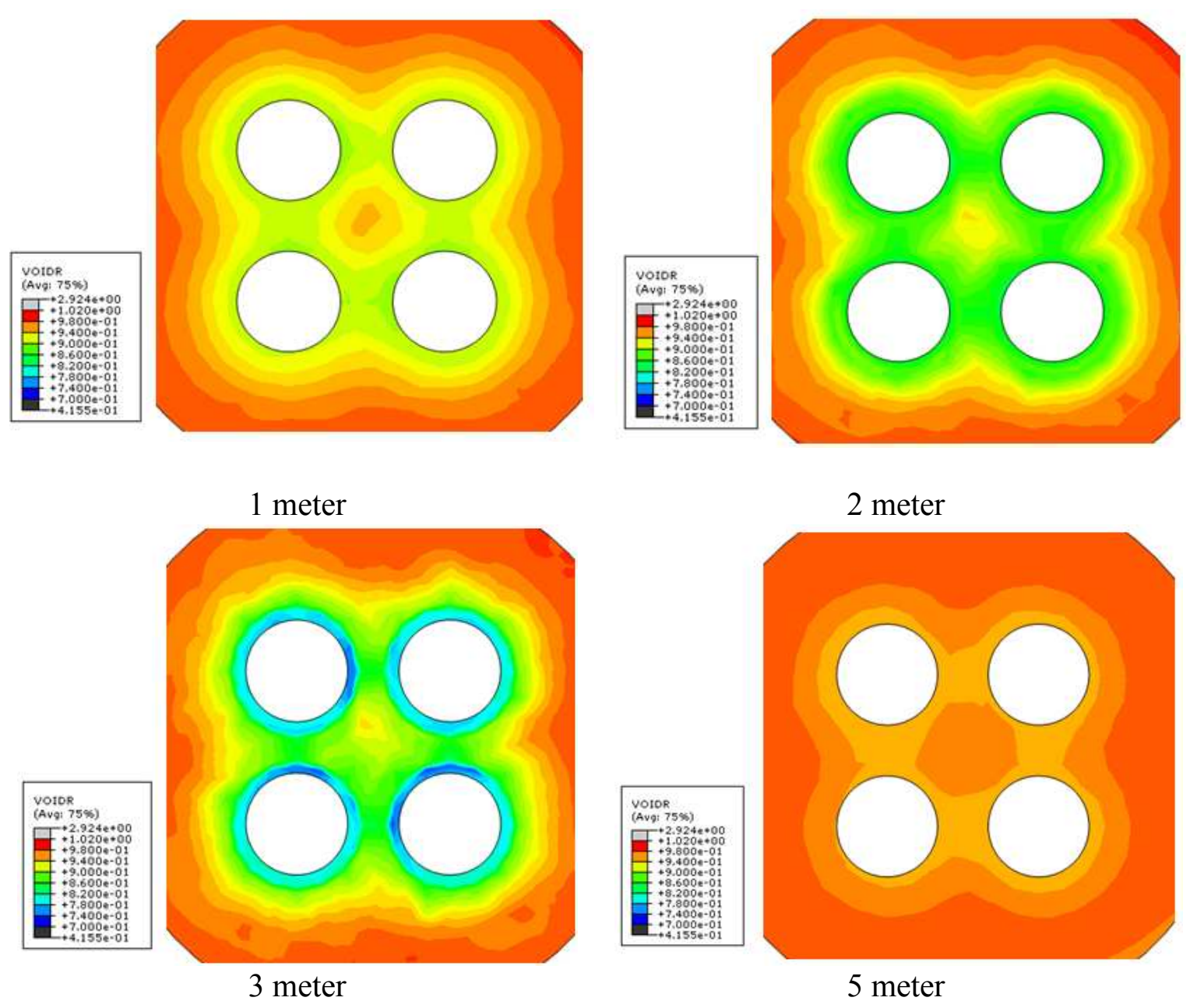

Figure 5-8 Horizontal cross sections (3R model)

Figure 5-9 and Figure 5-10 show two cross sections of the 3-D model results, plotted in terms of contours of void ratio distributions. The following observations can be noticed:

- Along the footprint side cross section, the interference between the nearby tamping is very clear.

- Along the foot print diagonal cross section, the interference is minimum since the diagonal distance is longer than the distance on the side 
- At the end of tamping, soil above the crater bottom level experience increase in void ratio.
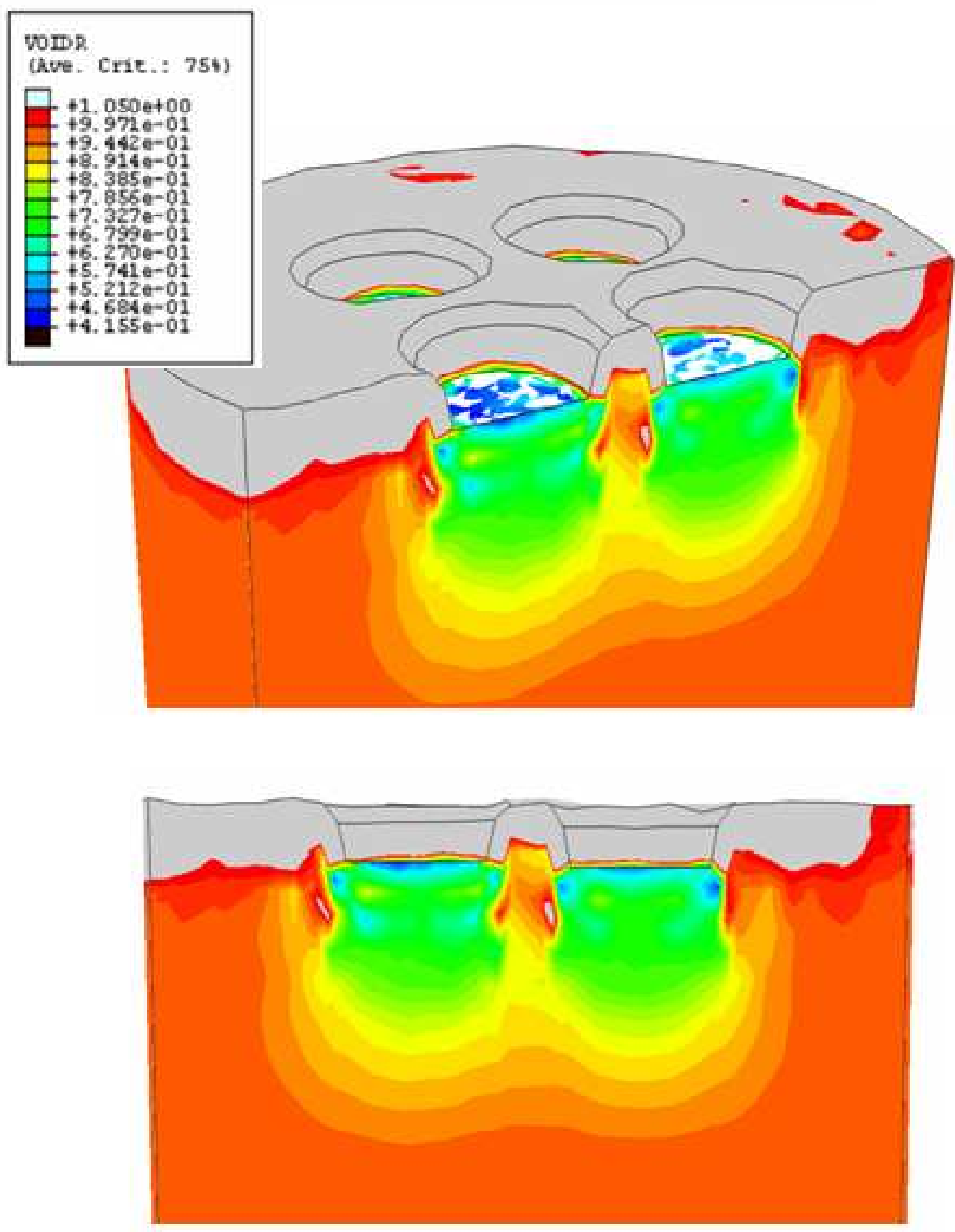

Figure 5-9 Interaction between the nearby tamping along footprint side (3R model) 

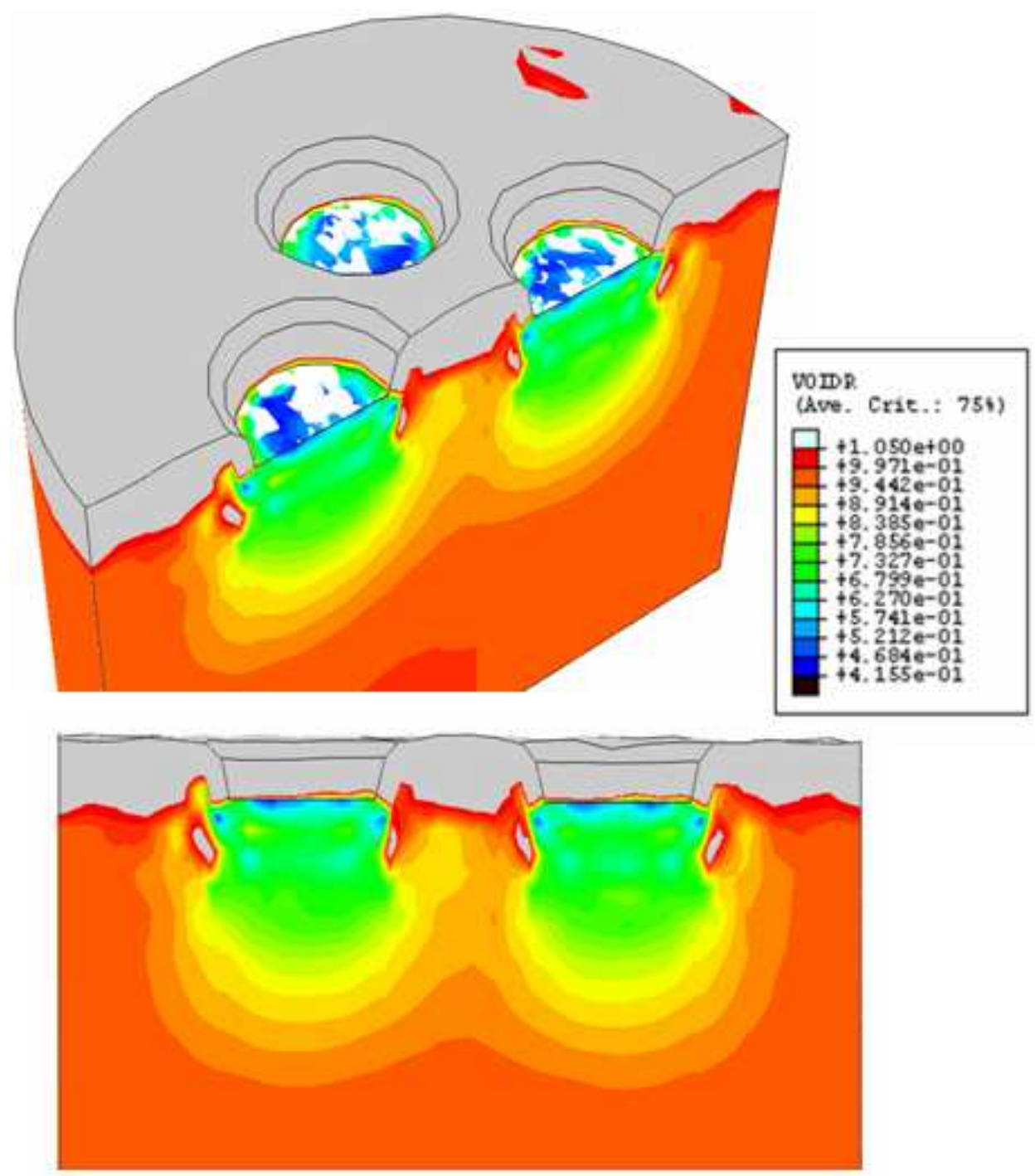

Figure 5-10 Interaction between the nearby tamping along footprint diagonal (3R model)

Void ratio distribution at Point $\mathrm{A}$ after the end of tamping at each spot is plotted and compared in Figure 5-11. The solid line in the plot represents the void ratio distribution (base distribution) directly beneath the tamper center after completing tamping at the four locations. A total of four lines were plotted in the figure to represent the void ratio distribution with depth beneath Point $\mathrm{A}$ at the end of four drops of tamper at each spot. Since the surface level of the soil differed from point to another because of the induced caters, the depth presented in the following plots is a measured from the 
original soil surface before tamping. Therefore, the void ratio beneath the tamper is shifted $1(\mathrm{~m})$ downward, which is equal to the induced crater depth, as can be seen from Figure 5-11.

After tamping at the first spot, an increase in void ratio is noticed. Slight change in void ratio was noticed after tamping at the second spot. Tamping at the third and the fourth spots does not lead to additional change in void ratio variation with depth beneath point A. This phenomenon could be explained as follows. Point A falls between spot 1 and spot 2; therefore, the change in void ratio beneath Point $\mathrm{A}$ is dominated by tamping at spot 1 and spot 2 which are closest to point A. At this grid spacing, tamping at spots 3 and 4 was found to have minimum influence on point $\mathrm{A}$. 


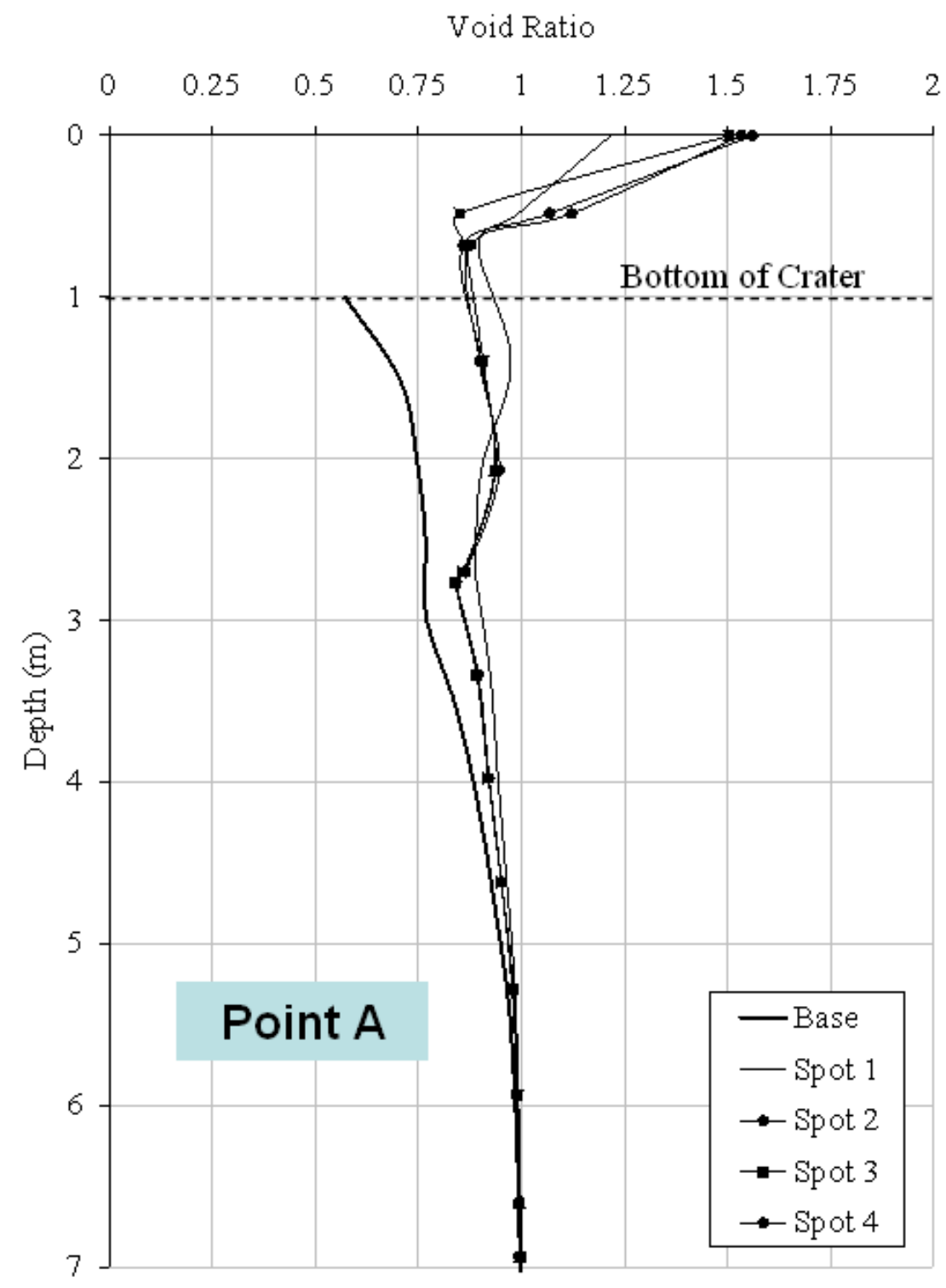

Figure 5-11 Void ratio distribution beneath point A after tamping at each spot (3R model)

Similarly, Figure 5-12 shows the void ratio variation with depth for point B, which is located at the center of the footprint spacing. The solid line represents the void ratio variation with depth beneath the tamper at the end of all tamping. Four other lines represent the void ratio variation with dept. Each line represents void ratio distribution beneath point B at the end of four drop at each spot. Since point B falls at the center of 
the four grid points, the void ratio beneath Point B was influenced by tamping at each of the four locations. It can be noticed at the end of tamping, the final reduction in void ratio beneath point $\mathrm{B}$ is about $30 \%$ of the reduction beneath the tamper. Point $\mathrm{B}$ is the least influence point during DC. Increase Void ratio distribution beneath point B and above the crater level is noticed.

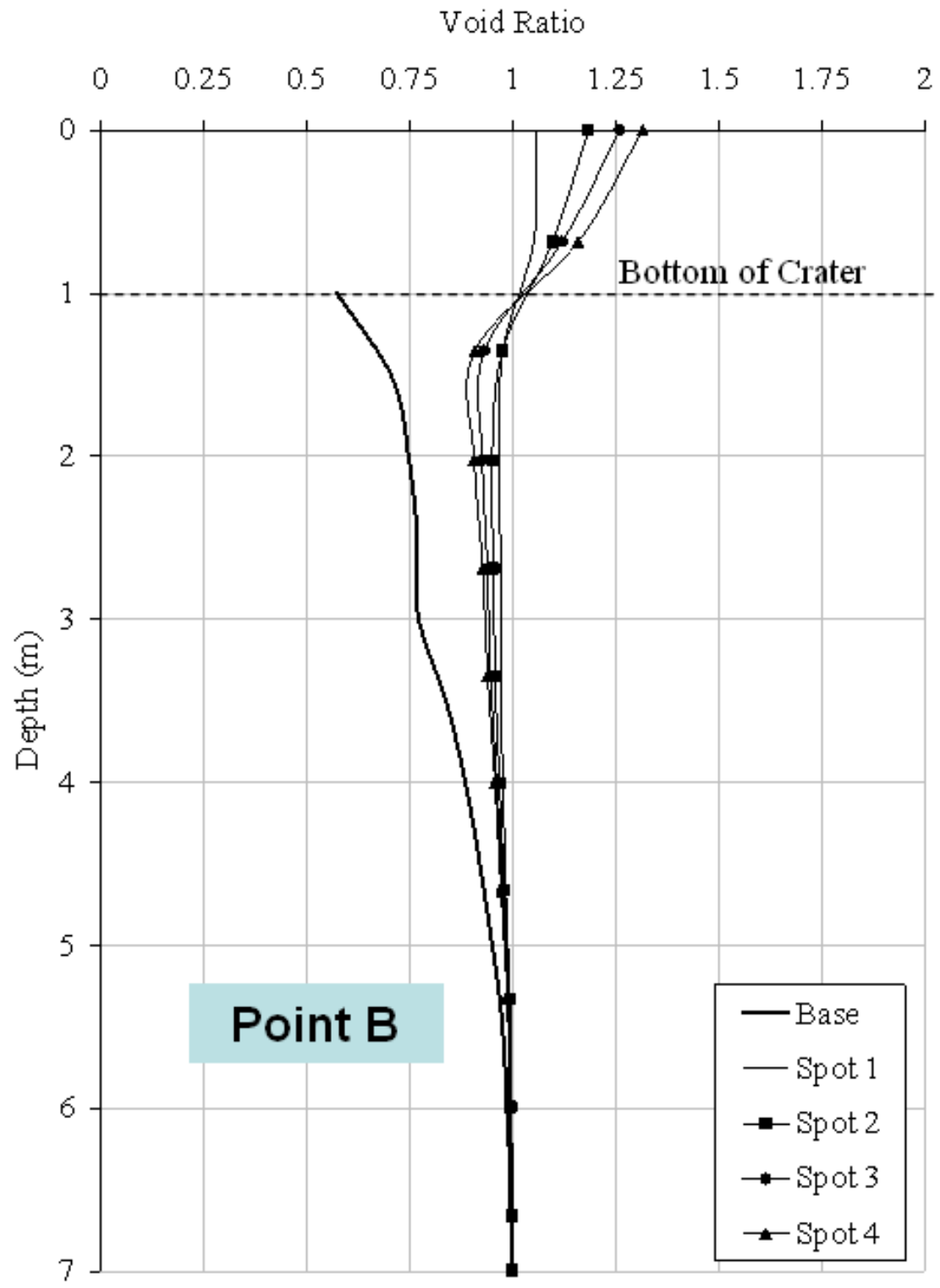

Figure 5-12 Void ratio distribution beneath point $\mathrm{B}$ after tamping at each spot (3R model) 


\subsubsection{Results for Model of 2.5R Spacing $(\mathrm{R}=1.25 \mathrm{~m})$}

In this model the footprint spacing is equal to $2.5 \mathrm{R}$; where $\mathrm{R}$ is the tamper radius.

Figure 5-13 shows cross sections at depth 1, 2, 3 and 5 meters measured from the bottom of the crater. The interaction between the nearby tamping spots can be clearly noticed. The center of the grid receives the least treatment along the depth. The treatment at the footprint side mid-point is strongly influence by the interaction between the adjacent tamping drops. the cross section plots shows the difference in interaction between $2.5 \mathrm{R}$ model and 3R model. For the $2.5 \mathrm{R}$ model, the interaction is higher and more consistent along the depth.

Figure 5-14 shows the variation of void ratio distribution along the footprint side. The interaction for this case is higher compared to the $2.5 \mathrm{R}$ model. Almost, a homogenous layer of the improved zone can be noticed. In addition, Figure 5-15 shows the void ratio distribution along the diagonal of the foot print. The interference is noticed to be higher than the similar case of $3 \mathrm{R}$ mode. 

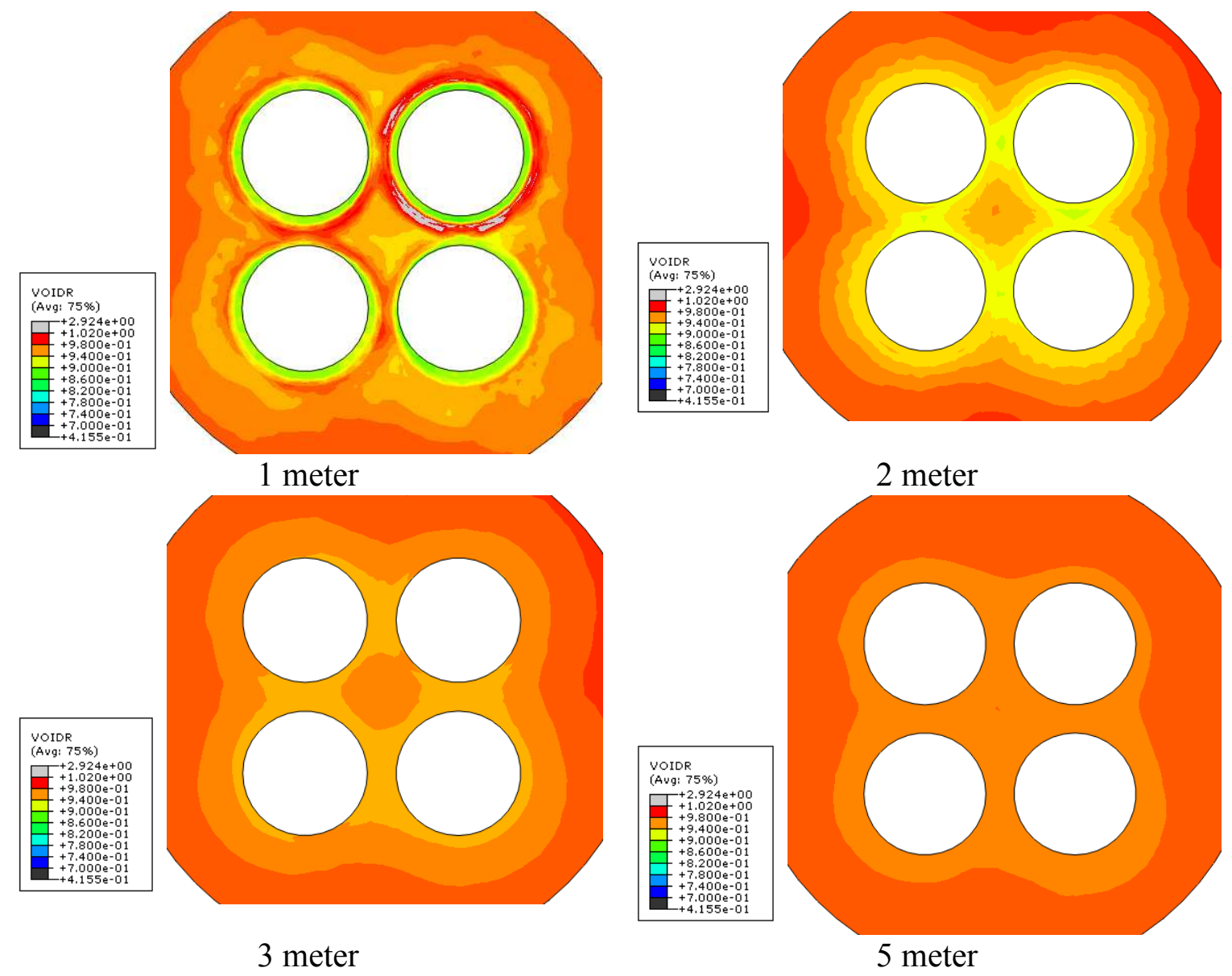

Figure 5-13 Horizontal cross sections (2.5R model) 


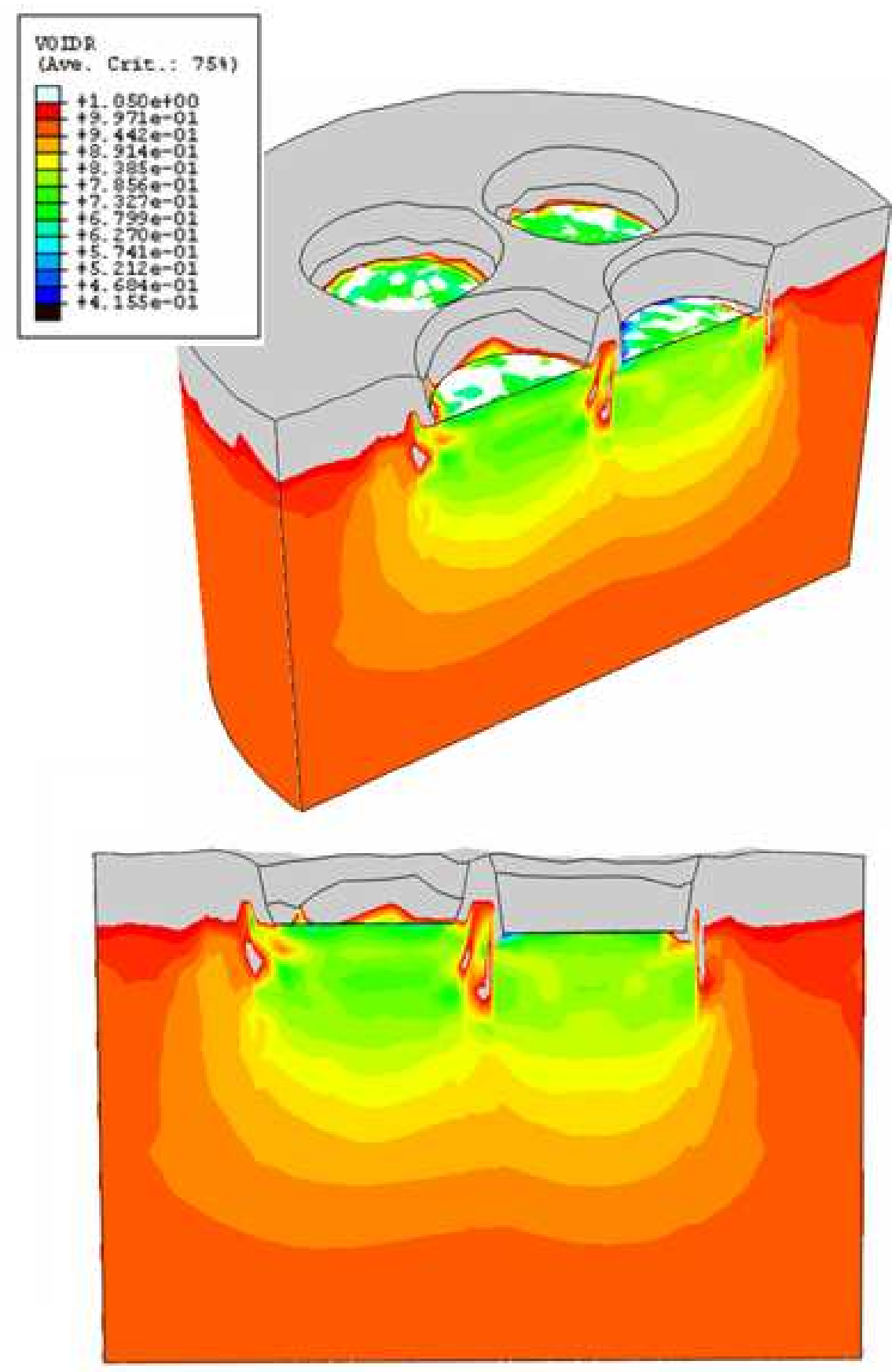

Figure 5-14 Interaction between the nearby tamping along footprint side (2.5R model) 

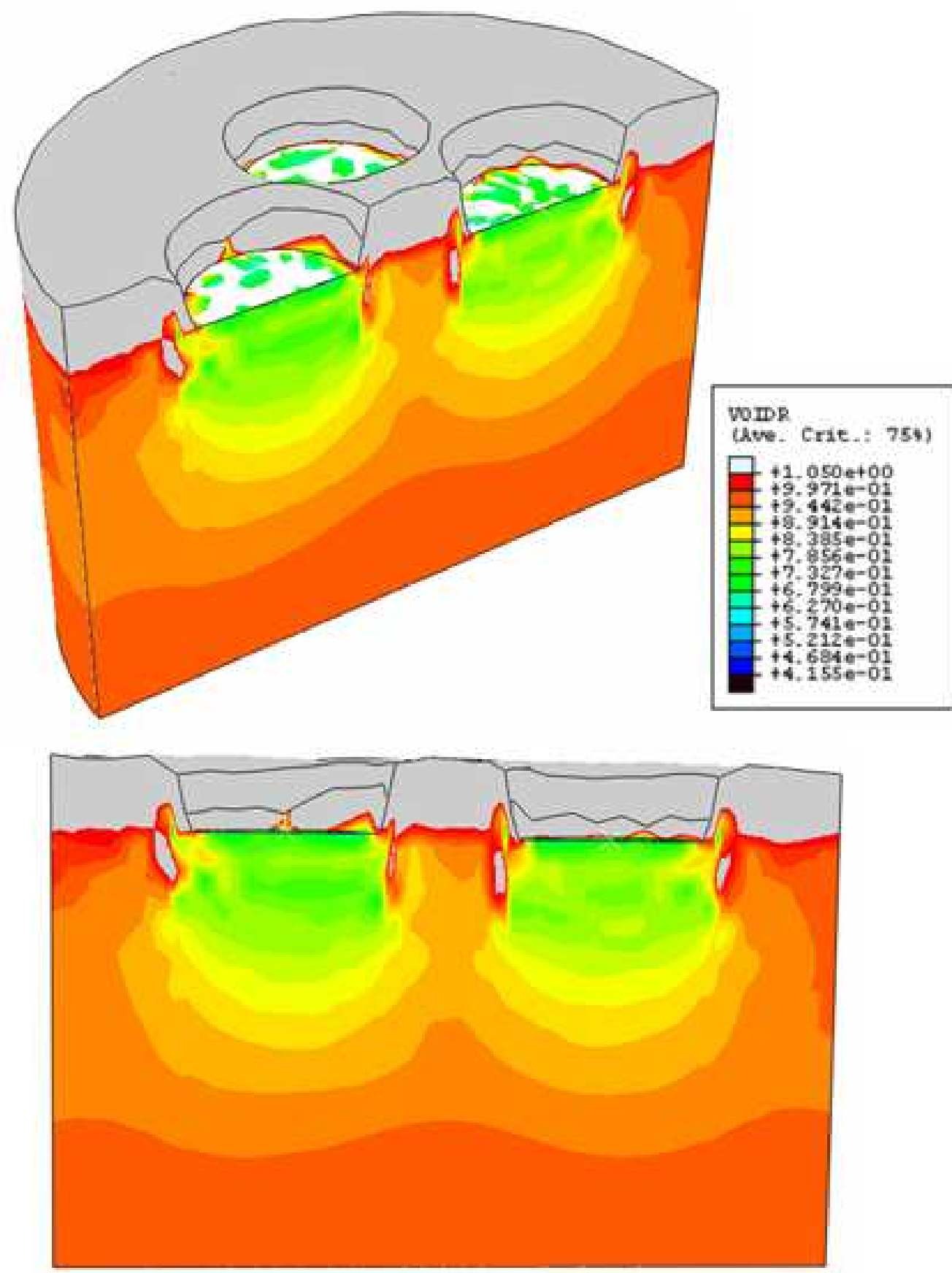

Figure 5-15 Interaction between the nearby tamping along footprint diagonal (2.5R model)

Similar to the plots plotted for $3 \mathrm{R}$ model, the void ratio variation after tamping at each spot for points A and B in the 2.5R Model are plotted in Figure 5-13 and Figure 5- 
14, respectively. Figure 5-16 shows that similar to $3 \mathrm{R}$ model; void ratio variation at Point A was affected by tamping at two spots nearest to the location of Point A. It can be noticed that, the void ratio beneath point $\mathrm{A}$ is close to the base void ratio distribution, which represented void ratio beneath the tamper center. Therefore, a grid spacing equal to $2.5 \mathrm{R}$ will result in treatment at the grid spacing sides equal to the treatment directly beneath the tamper.

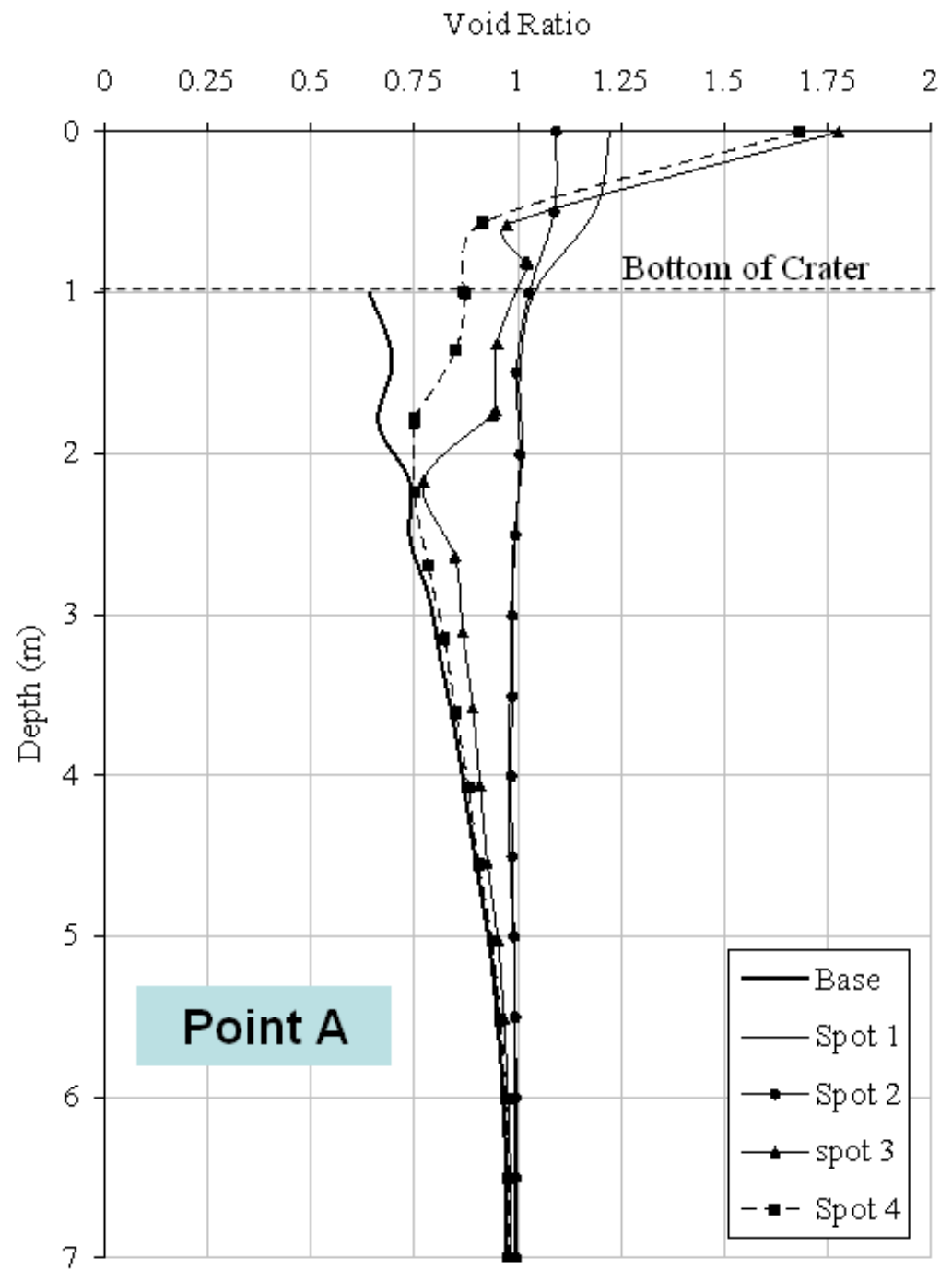

Figure 5-16 Void ratio distribution beneath point A after tamping at each spot (2.5R model) 
Figure 5-17 shows the void ratio variation with depth for point B for 2.5R model. It can be noticed that tamping in each spot results in nearly equal effect on the void ratio variation with depth at point $\mathrm{B}$. The reduction in void ratio at point B is about $50 \%$ for the reduction achieved beneath the tamper.

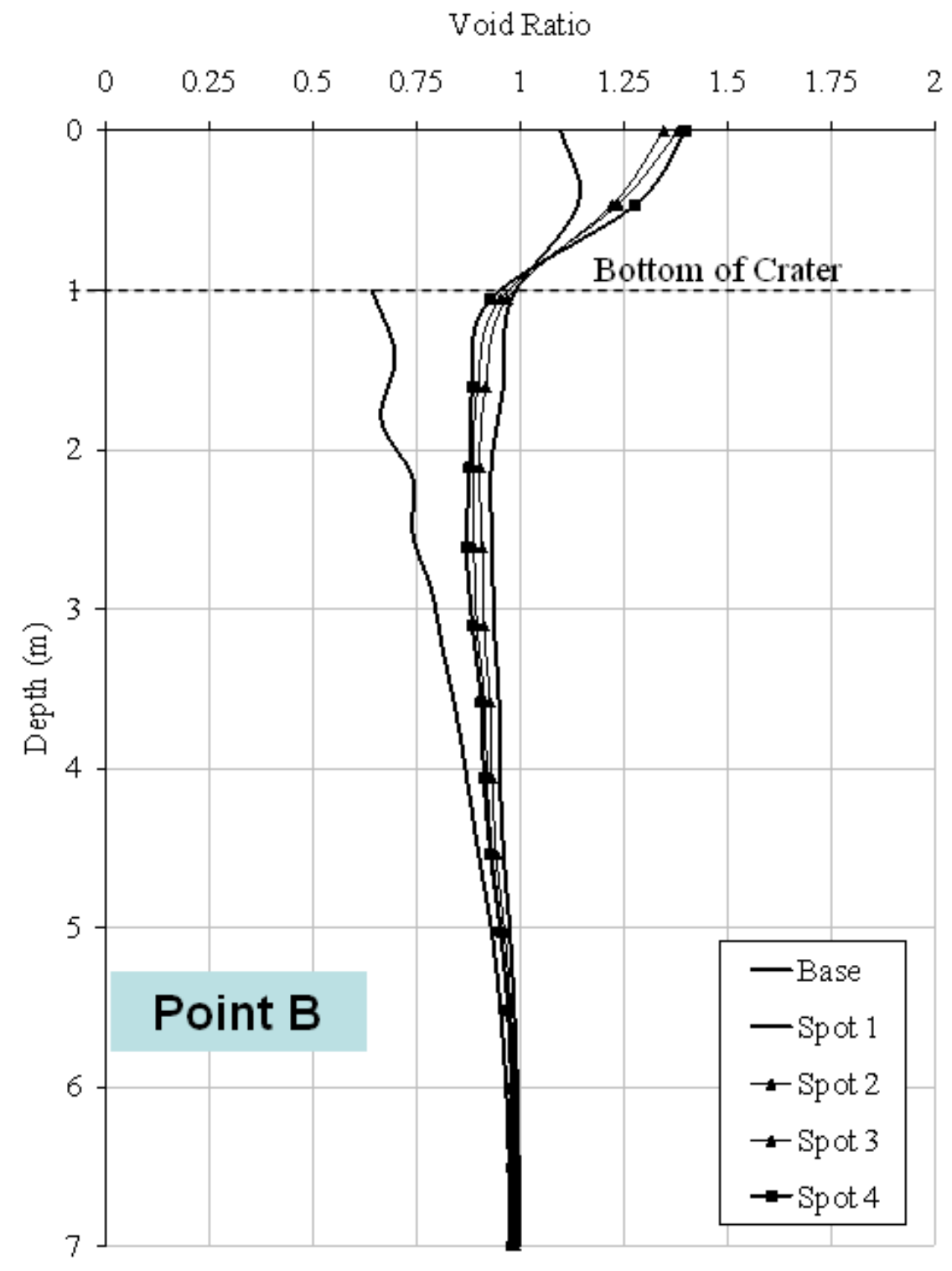

Figure 5-17 Void ratio distribution beneath point B after tamping at each spot (2.5R model) 


\subsection{Conclusion and Summary}

In this chapter, ABAQUS FE code was used to study the effects of multiple tamping from adjacent spots on the void ratio changes at two selected points. The CamClay constitutive model was adopted to represent the cohesive soil stress-strain-volume change behavior. Two 3-D FE models were developed: one represents grid spacing of 3R while the other one represents a grid spacing of $2.5 \mathrm{R}$. For the two model cases, four drops of a tamper weigh 10 ton and with drop height of 10 meter were simulated at each of the four tamping spots. Based on the FE simulation results, the following observations can be made.

- 3-D FE modeling of dynamic compaction in cohesive soils was conducted successfully

- The highly non-linear behavior of clay could be captured using Cam-Clay constitutive model.

- The interference between the adjacent spots was investigated by studying the void ratio distribution after completion of four drops of tamping at each spot.

- Based on the study presented in this chapter, a footprint grid of spacing of $2.5 \mathrm{R}$, where $\mathrm{R}$ is the tamper radius, seems to provide a more uniform treatment than the case of grid spacing of $3 \mathrm{R}$.

- If grid spacing of $2.5 \mathrm{R}$ was used, the resulting void ratio variation with depth at Point A (along the sideline of two connecting spots) will be close to those beneath the tamper. 
- Also, If grid spacing of 2.5R was used, the point at the center of footprint (Point B) will receive the least treatment; approximately $50 \%$ of the treatment beneath the tamper. 


\section{CHAPTER VI}

\section{DESIGN METHODOLGY}

\subsection{Introduction}

The work presented in the previous chapters was concerned with the numerical FE modeling of dynamic compaction in cohesive soil. Intensive parametric study was conducted to develop correlations among DC effects (i.e., crater depth, void ratio variations with depth, and the associated peak particle velocity) and the tamping energy as well as initial soil properties. The statistical analysis of FE simulation results showed that soil properties can exert strong influences on the DC effects. The use of those correlations requires specific soil properties which can be determined by site sampling, laboratory testing. Moreover, knowledge about Cam-Clay constitutive model parameters may become essential for the designer to understand the importance of each parameter. To simplify the use of the proposed correlations, a step-by-step design methodology for optimized DC operation in cohesive soils is presented in this chapter. New design charts to allow estimate of the relative change in soil density after dynamic compaction as a function of the induced crater depth and initial soil properties are presented. The method for estimating cost of DC based on the total number of drops required to treat a gived site was developed. 


\subsection{Crater Depth Effect on Void Ratio Distribution}

In chapter IV, the correlation relationship between the induced crater depth and the normalized tamper momentum as well as the total number of drops was derived. A correlation relationship parameter $\mathrm{C}$ was proposed and was found to be dependent on material properties (Cam -Clay Model parameters). In addition, a correlation relationship between the void ratio variation with depth beneath the tamper and the scaled energy of the tamper as well as the number of tamper drops at each spot was derived. Two correlation parameters, $\mathrm{C}_{1}$ and $\mathrm{C}_{2}$, were proposed to represent the influences of soil properties on the correlations. The use of the derived correlations depends on the ability of the designer to obtain the Cam-Clay constitutive parameters through laboratory testing. However, the mechanism of compaction and the relation between the induced carter depth and the degree of improvement can not be understood from the correlations. Therefore, further manipulation is presented herein to investigate the relation between the induced crater depth and the void ratio variation with depth.

In chapter IV, the following correlation equations were derived (i.e., Equations (4-1) and

$$
P=C\left(\frac{m V_{0}}{A}\right) \cdot \sqrt{N}
$$

And,

$$
\Delta e_{0}=C_{1} e^{-C_{2} y}\left(\frac{\sqrt{W H}}{A}\right) \cdot \sqrt{N}
$$

To investigate the change in void ratio as function of crater depth, Equation (4-4) is divided by (4-1) as follows: 
$\frac{\Delta e_{0}}{P}=\frac{C_{1} e^{-C_{2} y}\left(\frac{\sqrt{W H}}{A}\right) \sqrt{N}}{C\left(\frac{m V_{0}}{A}\right) \sqrt{N}}$

Since $V_{0}$ represents the striking velocity of the tamper mass which is dropped from height $H$, it can be calculated as follows:

$V_{0}=\sqrt{2 g H}$

Then, by simplifying Equation (6-1), one obtains the following equation.

$\frac{\Delta e_{0}}{P}=\frac{C_{1}}{C} \sqrt{\frac{1}{2 m g}} e^{-C_{2} y}$

The relative change in soil density after compaction is related to the void ratio before and after compaction. Since, Mass of the soil is constant before and after compaction:

$m_{1}=m_{2}$

Then,

$\rho_{1} V_{1}=\rho_{2} V_{2}$

$\rho_{1}\left(V_{\text {solid } 1}+V_{\text {void } 1}\right)=\rho_{2}\left(V_{\text {solid } 2}+V_{\text {void } 2}\right)$

where

$\rho_{1}, \rho_{2}$ : Soil density before and after compaction, respectively

$V_{1}, V_{2}$ : Soil volume before and after compaction, respectively

$V_{\text {solid } 1}, V_{\text {solid } 2}$ : Volume of solid in soil before and after compaction, respectively

$V_{\text {void } 1} \cdot V_{\text {void } 2}$ : Volume of voids in soil before and after compaction, respectively 
Since volume reduction occurs due to reduction in void volume, the volume of the solid volume is constant $\left(V_{\text {solid } 1}=V_{\text {solid } 2}\right)$. By dividing equation (6-6) by $V_{\text {solid } 1}$ :

$$
\rho_{1}\left(1+\frac{V_{\text {void } 1}}{V_{\text {solid } 1}}\right)=\rho_{2}\left(1+\frac{V_{\text {void } 2}}{V_{\text {solid } 2}}\right)
$$

Since,

$$
e_{1}=\frac{V_{\text {void } 1}}{V_{\text {solid } 1}} \text {, and } e_{2}=\frac{V_{\text {void } 2}}{V_{\text {solid } 2}}
$$

where

$e_{1}, e_{2}:$ void ratio before and after compaction, respectively

Then,

$$
\begin{aligned}
& \frac{\rho_{2}}{\rho_{1}}=\frac{1+e_{1}}{1+e_{2}} \\
& \frac{\rho_{2}}{\rho_{1}}=\frac{1+e_{1}}{1+e_{1}-\Delta e_{o}} \\
& \frac{\Delta e_{0}}{P}=\frac{C_{1}}{C} \sqrt{\frac{1}{2 m g}} e^{-C_{2} y}
\end{aligned}
$$

Finally, the relative density can be expressed as:

$$
\frac{\rho_{2}}{\rho_{1}}=\frac{1+e_{1}}{1+e_{1}-\frac{C_{1}}{C} \sqrt{\frac{1}{2 m g}} e^{-C_{2} y} \cdot P}
$$

Where $\mathrm{C}, \mathrm{C}_{1}$ and $\mathrm{C}_{2}$ where correlation constants previously defined in chapter IV as 


$$
\begin{aligned}
& C=\frac{1.8 e_{o}^{1.93} \lambda^{0.04}}{\rho^{0.56} M^{0.3} a_{o}^{0.53}} \\
& C_{1}=\frac{0.58 e_{o}^{1.4} \lambda^{0.9}}{M^{0.32} v^{0.2}} \\
& C_{2}=\frac{0.22 M^{.23} \lambda^{0.5} a_{o}^{0.5}}{e_{o}^{0.17}}
\end{aligned}
$$

Equation (6-11) presents the relative change in soil density as a function of soil properties; tamper mass, and the induced crater depth. The increase in crater depth results in decrease in the relative change in soil density; hence, better and deeper improvement is achieved. The importance of this correlation is that it represents a simplified technique to design and monitor DC operation. The degree of improvement and depth of improvement can be estimated for each soil type by monitoring the crater depth which can be simply measured during DC operation.

In order to understand the importance of Equation 6-10, Figure 6-1 shows the reduction in void ratio $\Delta e_{0}$ on the vertical axis versus the crater depth $\mathrm{P}$ on the horizontal axis. The plot was prepared using the baseline soil properties listed in Table (3-1). Each line in the plot represents the possible reduction in void ratio $\Delta e_{0}$ as a function of crater depth P. For example, for this soil parameters, if 1 meter crater depth was induced, this will lead to a reduction in the initial void ratio $\left(\Delta e_{0}\right)$ equal to $0.15,0.22,0.32$ and 0.45 at depths $2.0,1.5,1.0$ and $0.5(\mathrm{~m})$ respectively. It is noted that the depth is measured from the bottom of the crater.

The mechanism of compaction can be explained using Figure 6-1 as follows: 
- Soil before compaction is represented by point A

- Tamping results in a crater depth, from which reduction in void ratio is represented by the line $\mathrm{AB}$

- Additional Tamping causes more reduction in void ratio until $\Delta e_{0}(\max )$ is achieved for that depth which is presented as point B

- The maximum possible treatment has been achieved at point B. additional tamping will result in increase in crater depth and hence increase in the zone which has the maximum possible treatment. This can be explained by point $\mathrm{C}$. The line $\mathrm{BC}$ represented the growth of the zone of maximum possible improvement.

So far the case of multiple rounds of tamping was not discussed. One of the main advantages of Figure 6-1 is that it can also be used to design for a second round of tamping. An example is shown in Figure 6-1. Assume that soil has been improved to $\Delta e_{0}(\max )$ down to certain depth, for example 1 meter, which is represented, by point $\mathrm{B}$; however, additional treatment is required to deepen the maximum depth of improvement to 1.5 meter. A crater depth equal to $\mathrm{BC}$ is required to achieve the objective of the second round of tamping. 


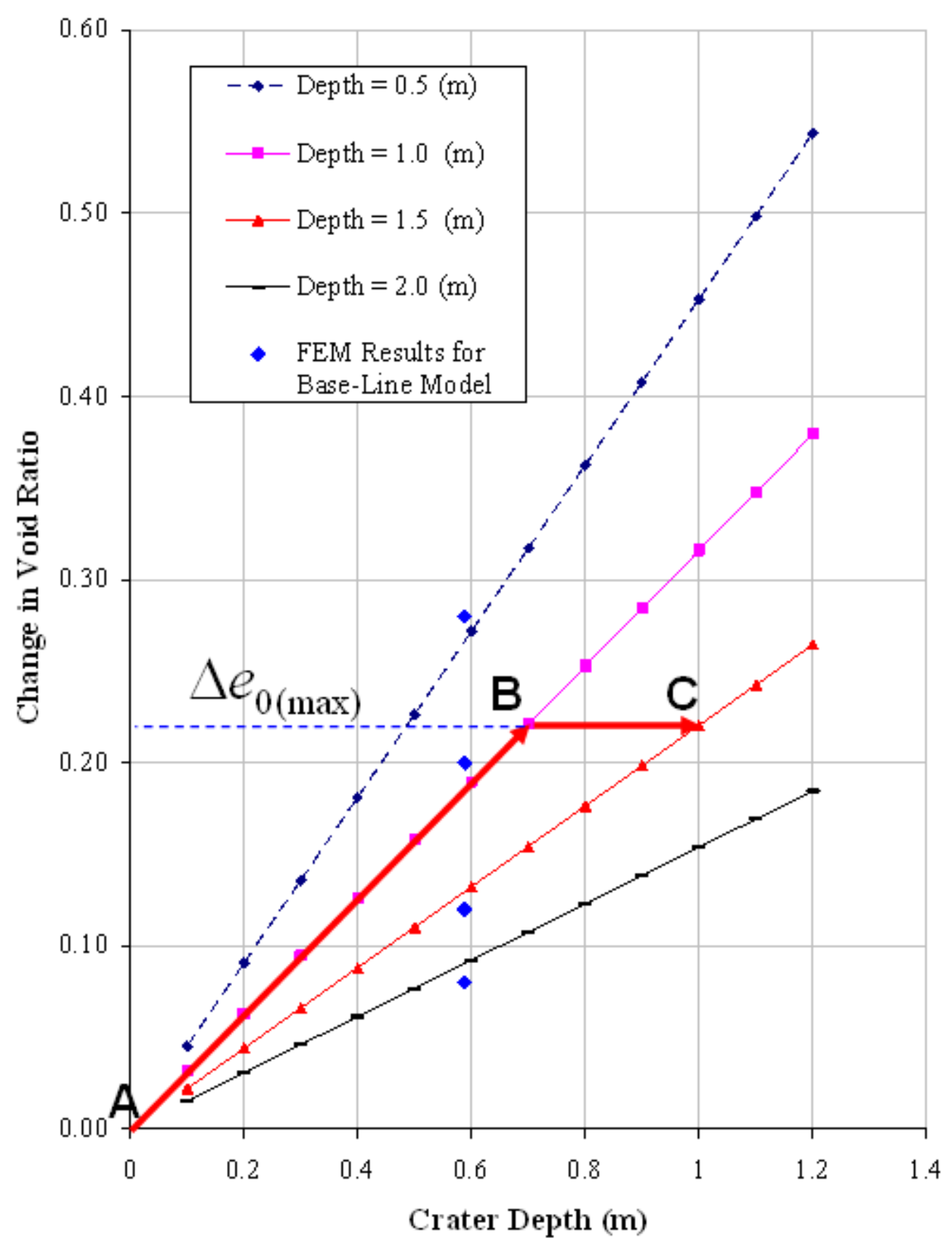

Figure 6-1 Void ratio as a function of crater depth

\subsection{Classification of Soil Suitability for DC}

The correlations presented in this dissertation were based on intensive parametric study in which the constitutive relationship of the cohesive soils is described by Cam- 
Clay model parameters. However, the use of these correlations requires laboratory testing to obtain the values for the six Cam-Clay model parameters $\left(\rho, v, M, e_{o}, \lambda, a_{o}\right)$. Moreover, the design engineer needs to be familiar with soil behavior and the influence of each parameter on the process of DC. To make the work presented in this dissertation more practical, an investigation was made to assess the soil suitability for DC.

In Equation (4-1), correlation between the induced crater depth and the normalized tamper momentum was presented. The Parameter $\mathrm{C}$ was selected to be the proportionality constant and was found to be dependent upon soil properties. Also from the analysis results in Chapter IV, the value of the parameter C falls between 0.01 and 0.034. Soils with high $\mathrm{C}$ value are more suitable for $\mathrm{DC}$, since high values of parameter $\mathrm{C}$ result in deep crater depth while low values of parameter $\mathrm{C}$ result in relatively shallow crater depth. Therefore, the parameter $\mathrm{C}$ can be used to classify the soil suitability for DC. Table 6-1 shows the value of parameter $\mathrm{C}$ and the corresponding values for parameter $\mathrm{C}_{1}$ and $\mathrm{C}_{2}$. One additional point in the middle of the range for $\mathrm{C}$ was added in the table, which represents soils with moderate suitability for treatment using DC. The fourth column is the reciprocal of $\mathrm{C}$ parameter which was found to be easier for manipulation.

Table 6-1 Values of parameters $\mathrm{C}, \mathrm{C}_{1}$ and $\mathrm{C}_{2}$

\begin{tabular}{c|c|c|c|c}
\hline Suitability for DC & $\mathrm{C}$ & $\mathrm{C}_{1}$ & $\mathrm{C}_{2}$ & $\sim 1 / \mathrm{C}$ \\
\hline High & 0.034 & 0.239 & 0.677 & 29 \\
\hline Moderate & 0.020 & 0.173 & 0.771 & 50 \\
\hline Low & 0.010 & 0.096 & 0.757 & 100 \\
\hline
\end{tabular}


The parameter $\mathrm{C}$ can be obtained by conducting a laboratory test to obtain CamClay constitutive parameters correlation Equation (4-3) can then used to calculate the value of the parameter $\mathrm{C}$.

The parameter $\mathrm{C}$ can be also obtained through simple field test. In the field test, tamper with mass $(m)$ and cross section area (A) can be dropped from height $(H)$ to strike soil at velocity $\left(V_{\mathrm{o}}\right)$. The impact may be repeated $(\mathrm{N})$ times. A crater with depth $(\mathrm{P})$ will be induced. Based on Equation (4-1), the parameter 1/C can be calculated as follows:

$$
\frac{1}{C}=\frac{\left(m V_{0}\right) \sqrt{N}}{P A}
$$

By conducting a simple field test the parameter $\mathrm{C}$ can be obtained. Depending on the value of parameter $\mathrm{C}$, soil suitability can be determined using Table 6-1.

It is also possible to utilize the test conducted in the field to estimate the maximum possible improvement. The ratio $\rho_{2} / \rho_{1}$ can be obtained by measuring soil density before and after test. The importance of the density ratio will be explained in the following section.

\subsection{Design Charts}

In this section, values for $\mathrm{C}, \mathrm{C}_{1}$ and $\mathrm{C}_{2}$ that were presented in Table 6-1 are used to develop three design charts for DC in cohesive soils. Since three levels of soil suitability for DC was proposed, three design charts were developed. Figure 6-1 to Figure 6-4 show the relative change in the ratio of $\rho_{2} / \rho_{1}$, where $\rho_{2}$ and $\rho_{1}$ are soil density after and before DC, as a function of crater depth. Each chart is identified by specific 1/C value. Also, Equation (4-6), which was developed to estimate the Peak Particle Velocity 
(PPV) as a function of soil properties and the scaled tamping energy, can be used to find the corresponding PPV for the three values of 1/C. It is interesting to note that low level of PPV is noticed for soils with high suitability for DC.

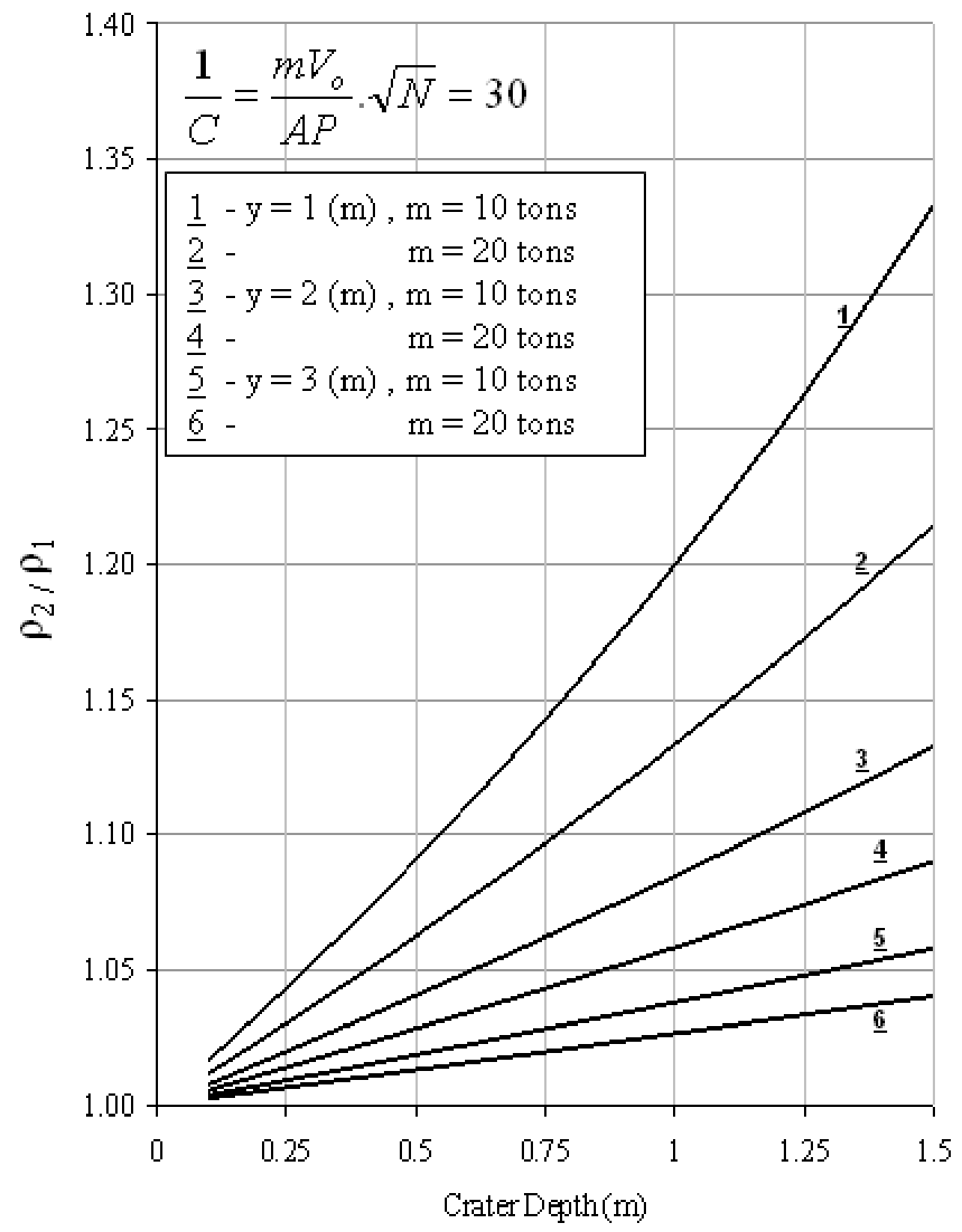

Figure 6-2 Design Chart for $1 / \mathrm{C}=30$ 


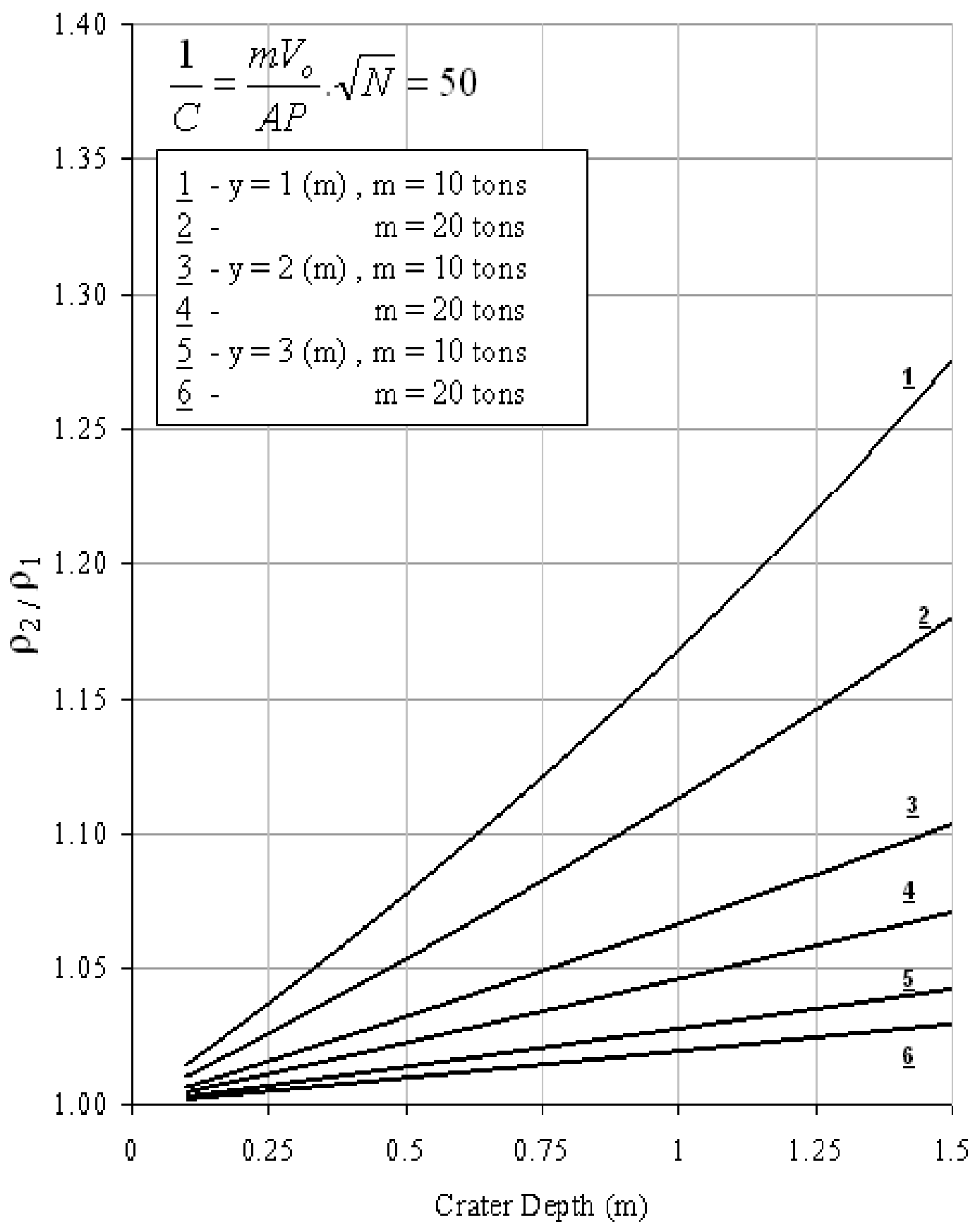

Figure 6-3 Design Chart for $1 / \mathrm{C}=50$ 


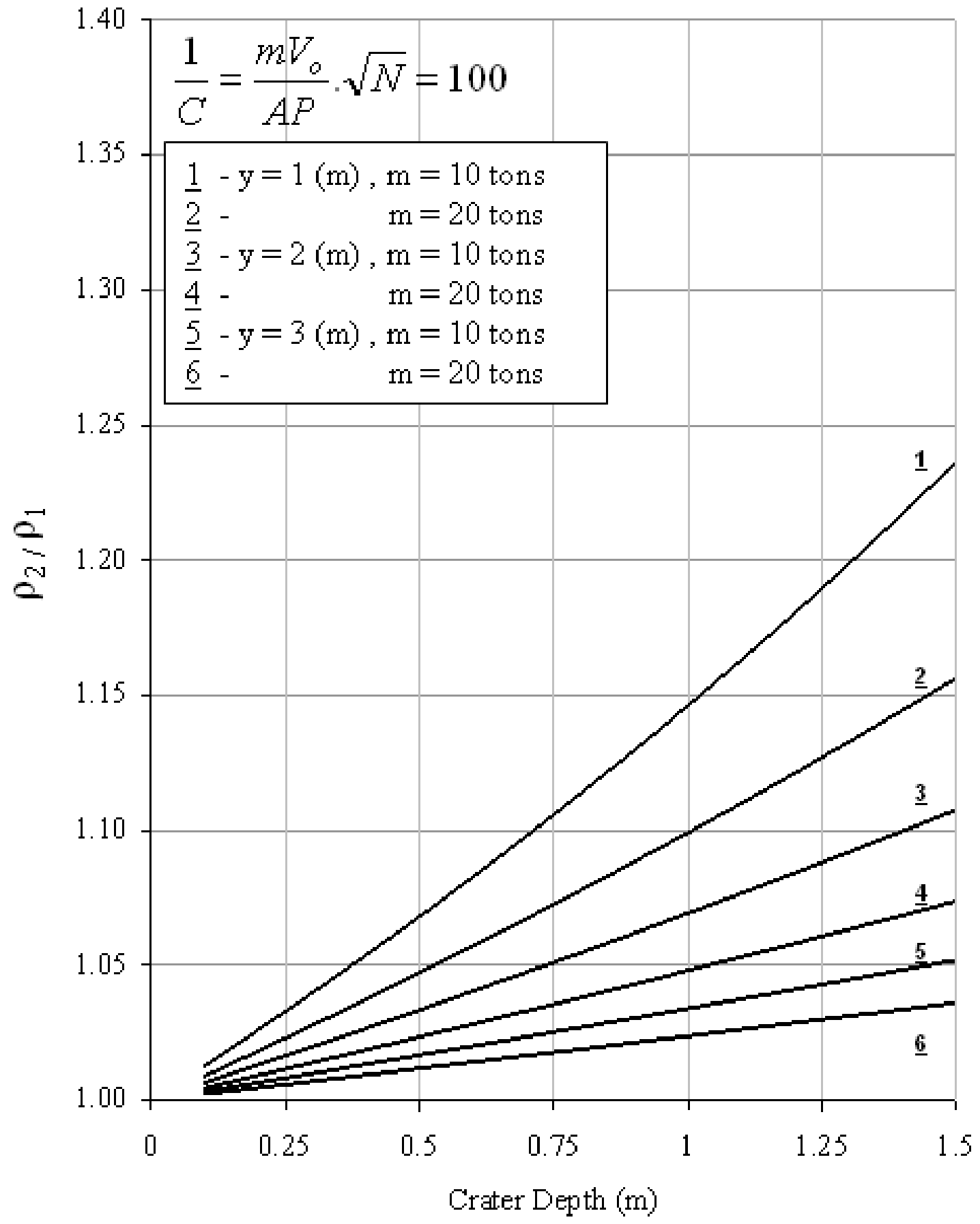

Figure 6-4 Design Chart for $1 / \mathrm{C}=100$ 


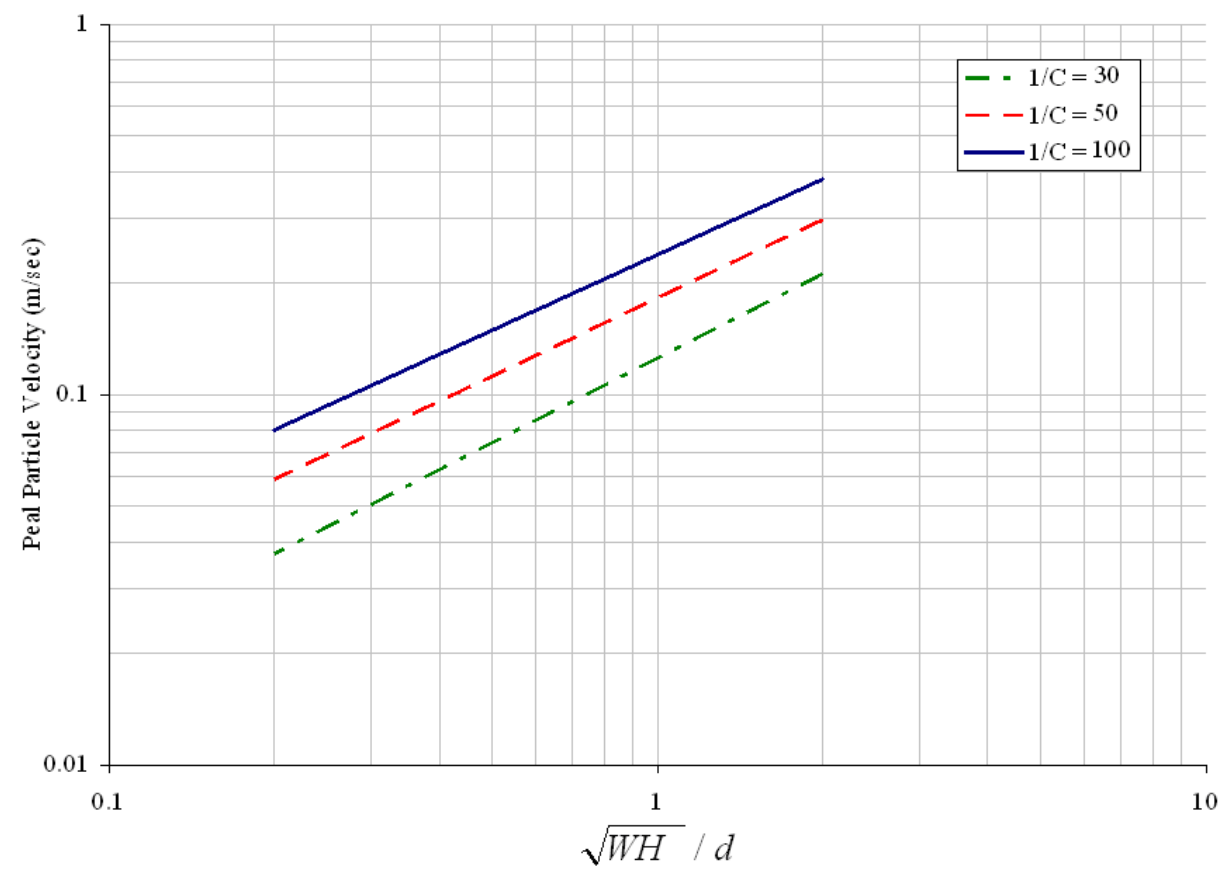

Figure 6-5 PPV design chart

\subsection{Cost of Dynamic Compaction}

The cost of dynamic compaction depends on the initial soil condition and the desired level of improvement. In this section, the correlations derived in pervious sections were used to provide a means for estimating the cost of dynamic compaction as a function of tamper characteristics and soil properties.

Assuming that one round (or one pass) of tamping is used at a site, then the total number of drops that is used to treat a site is equal to

$N_{T}=N_{\text {spots }} \cdot N$

Where

$N_{T}$ : Total Number of drops

$N$ : Number of drops at each spot. 
$N_{\text {spots }}:$ Number of spots at the site

The total number of tamping spots is equal to the site area divide by the footprint spacing grid squared $\left(S^{2}\right)$ :

$$
N_{\text {spots }}=\frac{A_{\text {site }}}{S^{2}}
$$

Also, utilizing Equation (4-1) the number of drops per spot can be calculated as follows:

$$
N=\frac{P^{2} A^{2}}{C^{2}\left(m V_{o}\right)^{2}}
$$

Substitute Equation (6-14) and Equation (6-15) into Equation (6-13)

$$
N_{T}=\frac{A_{\text {site }}}{S^{2}} \frac{P^{2} \cdot A^{2}}{C^{2}\left(m V_{o}\right)^{2}}
$$

The grid spacing for best interaction between the nearby drops occurs when,

$$
S=2.5 R
$$

And, the tamper cross section area can be calculated as.

$$
A=\pi R^{2}
$$

Finally, by substituting into Equation (6-7), the total number of drops per site can be calculated as

$$
N_{T}=A_{\text {site }} \frac{1.6 P^{2} \cdot R^{2}}{C^{2}\left(m V_{o}\right)^{2}}
$$

Equation 6-19 estimates the total number of drops $\left(N_{T}\right)$ needed to treat a site with total area $A_{\text {site }}$ when the grid spacing was selected to be $S=2.5 R$. The cost of treating 
certain site increases linearly with the site area. It also increases with the induced crater depth squared and the tamper radius squared. On the other hand, the cost decreases with the tamper momentum squared and the parameter $C$ squared. It should be mentioned that the parameter $C$ was used earlier to measure soil suitability to treatments. Lower $C$ value indicates high suitability for DC treatment.

\subsection{Design Methodology.}

The following steps summarize the proposed design methodology.

1. Conduct filed test to identify the soil parameter $\mathrm{C}$ and the density ratio $\rho_{2} / \rho_{1}$. where

$\rho_{2}$ : Soil density at the crater bottom after tamping $\rho_{1}$ : Soil density at the crater bottom before tamping

2. Identify the maximum tolerable PPV

- This can be identified by investigating the condition of the surrounding structures. The local codes for maximum allowable PPV should be also reviewed.

3. Use Figure 6-5 to obtain $\sqrt{W H}$, which would correspond to the maximum tolerable PPV identified in Step 2 and C value identified in Step 1.

4. Usually cranes have fixed capacity to lift certain mass; therefore, the tamping energy is controlled by changing the drop height. Consequently, select the drop height that satisfies $\sqrt{W H}$ value obtained from Step 3 . 
5. Using the value of parameter $\mathrm{C}$, identify the appropriate design chart from Figure 6-2 to Figure 6-4. The crater depth at which the maximum relative density can be achieved down to a specified depth is selected.

6. Estimate the total number of drops to treat the site and estimate the cost using Equation 6-19.

7. Conduct the DC operation over grid spacing equal to 2.5 times the tamper radius.

\subsection{Step-by-Step Example}

Assuming a site 100 meter in length and 100 meter in width to be treated using DC. A tamper of mass 10 tons is available for use for the project. The tamper radius is 1.25 meter with the cross section area of $4.9 \mathrm{~m}^{2}$. The following steps demonstrate in details the process of using the proposed design charts.

1. Conduct a field test to identify the soil parameter $\mathrm{C}$ and the density ratio $\rho_{2} / \rho_{1}$.

The crane was used to conduct the field test. Suppose that 3 drops would have resulted in 1 meter crater with the tamper dropping from 10 meter high. Then, the parameter $\mathrm{C}$ can be calculated using Equation 6-12)

$$
\frac{1}{C}=\frac{\left(m V_{0}\right) \sqrt{N}}{P A}=\frac{(10 \text { (tons }) 14(\mathrm{~m} / \mathrm{sec}) \sqrt{3}}{1(\mathrm{~m}) 4.9\left(\mathrm{~m}^{2}\right)}=49.5
$$

Also, field measurement of the relative soil density was assumed as follows:

$$
\frac{\rho_{2}}{\rho_{1}}=\frac{2000\left(\mathrm{~kg} / \mathrm{m}^{3}\right)}{1800\left(\mathrm{~kg} / \mathrm{m}^{3}\right)}=1.11
$$


Based on these calculations from field measurements, Figure 6-3 is the closet chart for design for the soil conditions at this site.

2. Select the mass and drop height combination

Assume that the maximum tolerable $\mathrm{PPV}$ is equal to $0.1 \mathrm{~m} / \mathrm{sec}$ at a distance of 30 meter from the point of impact. DC operation was design not to affect any building at distance 30 meter and beyond. From Figure 66 the scaled energy of the tamper operation for this case is equal to 0.4

$$
\frac{\sqrt{W H}}{d}=\frac{\sqrt{10(\text { tons }) x H}}{30}=0.4
$$

Then,

$$
H=\frac{(0.4 \times 30)^{2}}{10}=14.4(\mathrm{~m})
$$

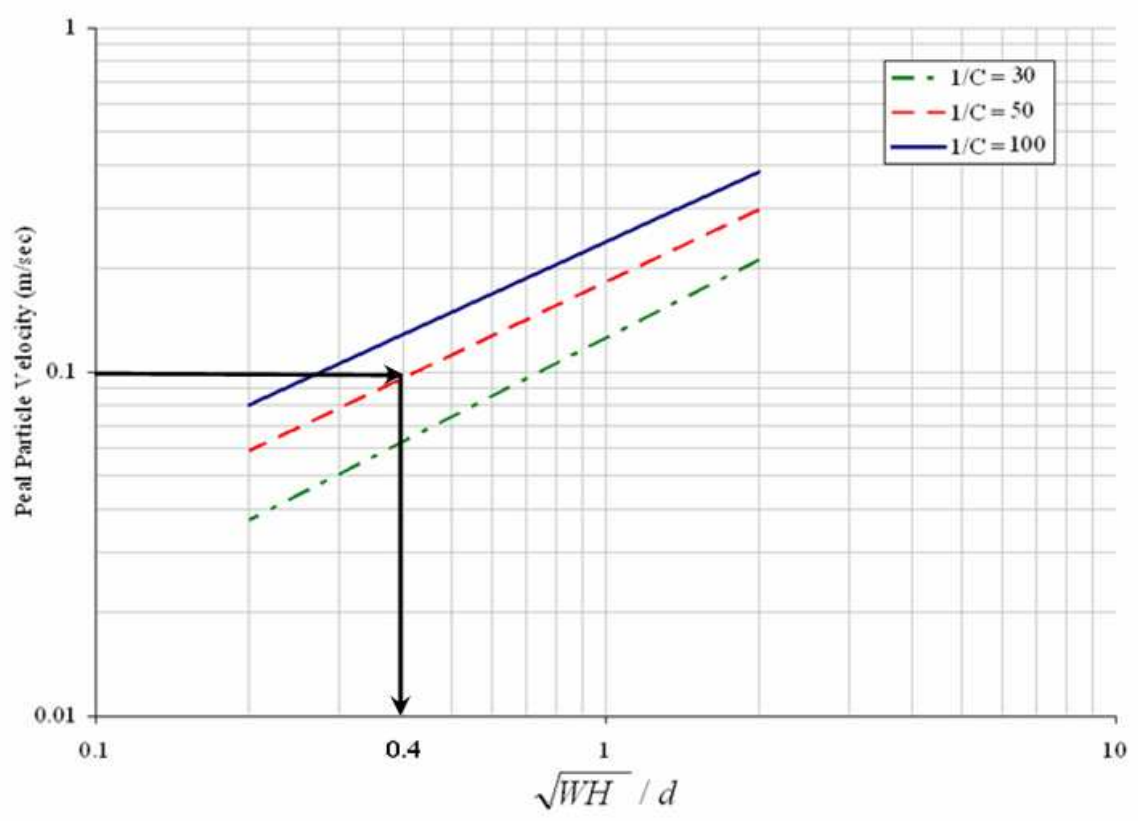

Figure 6-6 Selection of tamper mass and drop height 
3. Identify the required crater depth

Using Figure 6-3, horizontal line at $\rho_{2} / \rho_{1}$ equal to 1.11 is drawn. As illustrated in Figure 6-7, the interactions with line which represents a tamper of 10 tons mass are the points of interest. It can be noticed that to have the maximum possible improvement down to 2 meters, a crater depth of 1.5 should be induced. Also, if a crater depth equal to 0.6 meters, the maximum improvement is achieved down to 1 meter. For the later case, the density ratio at 2 meters is 1.05 compare to 1.11 at depth 1 meter.

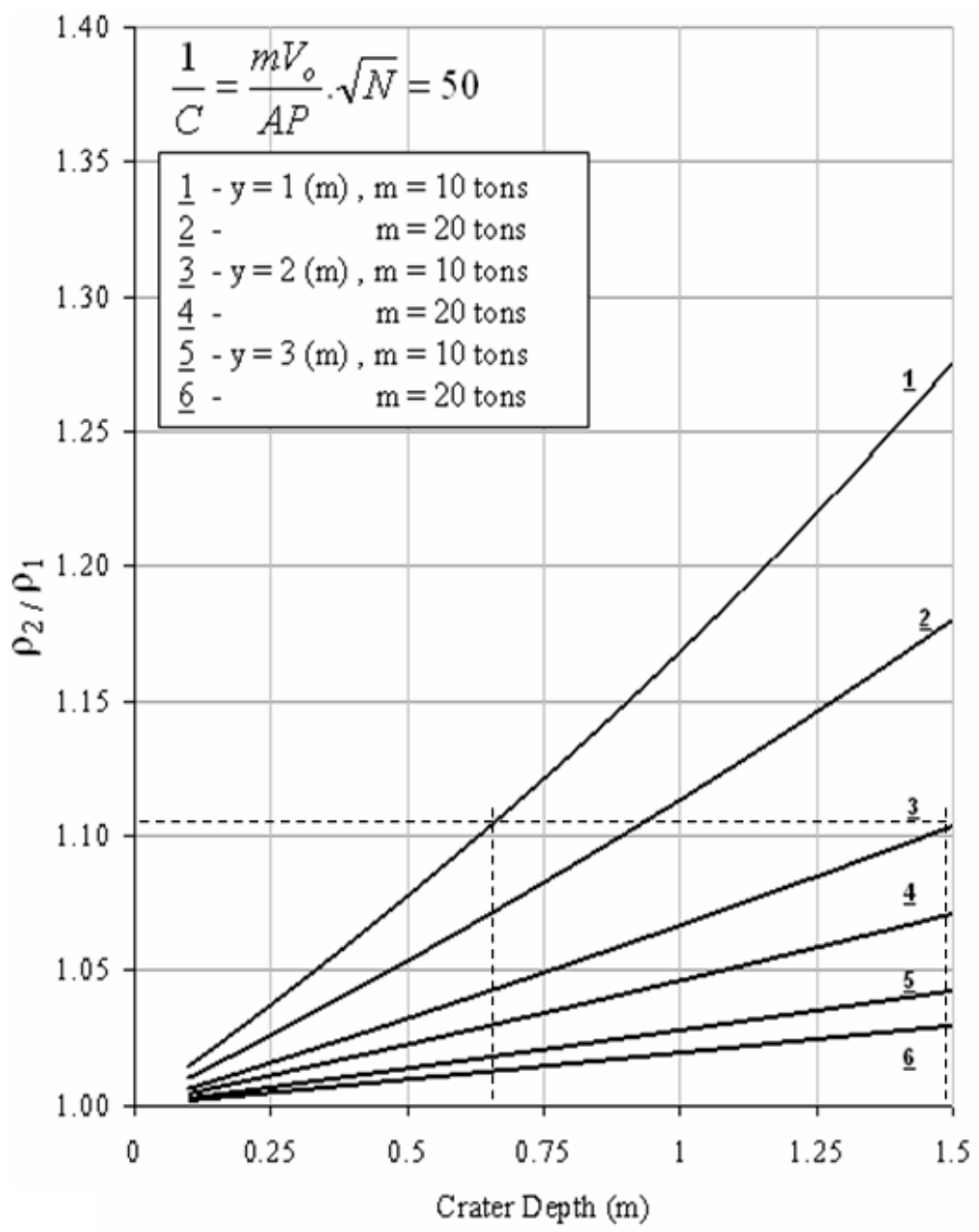

Figure 6-7 Identify the required crater depth 
4. Estimate the total number of drops

The total number of drops for the studied site can be estimated using Equation (6-15) as follows:

$$
N_{T}=A_{\text {site }} \cdot \frac{1.6 P^{2} R^{2}}{C^{2}\left(m V_{o}\right)^{2}}=(100 \times 100) x \frac{(1.6)(1.5)^{2}(1.25)^{2}}{(0.02)^{2}(10 \times 16.8)^{2}}=4984
$$

\subsection{Summary and Conclusions}

Three correlation equations to estimate the crater depth, void ratio variation with depth, and the peak particle velocity were presented in chapter IV. The use of these correlation equations, however, requires laboratory testing to identify the parameters of Cam-Clay constitutive model. Also, the use of these correlations requires computational efforts and good understanding of Cam-Clay constitutive model parameters. Therefore, in this chapter, simple design charts were developed to provide insights on the influences of soil properties on DC process as well as to facilitate more systematic design process for a specific site condition. The contributions of this chapter can be summarized as follows.

1. Correlation between density ratio and the induced crater depth was derived based on the correlations presented in chapter IV

2. A simple field test was proposed to estimate site specific soil parameter $\mathrm{C}$

3. Based on the field measured value of the parameter $C$, site specific soils can be classified for its suitability for DC

4. Design charts for three levels of soil suitability were developed to determine the required crater depth for which the maximum improvement can be achieved down to the desired depth 
5. Additional design charts were developed to estimate the PPV in correspondence with field measured and site specific value of parameter C.

6. The total number of drops for treating a site can be estimated based on the correlation equations presented in this chapter.

7. A practical design methodology was developed to allow for a systematic design approach to ensure effective and safe DC operation for a given site soil condition.

8. Finally, a step-by-step example was presented to illustrate the appropriate use of the developed design charts and to elucidate the selection process of tamper parameters and $\mathrm{QA} / \mathrm{QC}$ measures. 


\section{CHAPTER VI}

\section{SUMMARY AND CONCLUSIONS}

\subsection{Summary}

Toward the objective of developing a design methodology for the dynamic compaction in cohesive soils, techniques for numerical simulation of $\mathrm{DC}$ in cohesive soils characterized by the Cam-Clay constitutive model as well as extensive numerical parametric simulation work have been carried out in this dissertation. Detailed literature review was performed to review the existing empirical design models for dynamic compaction. Literature review indicated that knowledge on DC in cohesive soils was lacking and that further research in to this area was needed. A new design methodology for dynamic compaction in cohesive soils was developed and presented in this dissertation work.

The problem of dynamic compaction was studied numerically using ABAQUS FE code. The built in Cam-Clay constitutive model was used to represent soil continuum in infinite half space. The 2-D axi-symmetric model was developed for DC process to take advantage of the circular shaped tamper mass. The initial (geostatic) state of stress in the soil was defined prior to commencing simulation of tamper impact on the ground surface. The tamper was modeled using rigid elements and the tamper mass was modeled using concentrated mass at the center of the rigid tamper. The FE modeling techniques 
developed in this dissertation was validated using field data in terms of crater depth and PPV values for both cohesive and cohesionless soils.

The influence of tamping energy as well as each parameter of Cam-Clay constitutive model for cohesive soils on the DC effects was studied through an extensive FE parametric study. Furthermore, a new modeling technique involving the use of cutting a groove around the tamper was developed in this dissertation to study the case of multiple drops at the same spot. The model was able to successfully model six consecutive drops at the same spot. Based on the results from the extensive FE parametric study, three correlation equations were proposed to estimate the induced crater depth, the void ratio variation with depth, and the induced wave Peak Particle Velocity (PPV).

The effect of multi impact from the adjacent spots was studied using a 3-D FE model. In this study, soil continuum was modeled as a soil cylinder which has fixed boundaries. The cylinder dimensions were selected carefully to avoid interference from the fixed boundaries. The model consists of four tamping spots, with four drops per spot, resulting in a total of 16 drops in the entire simulation. The model was tamped using rigid disc which simulates the tamper action with force-time function determined from a model using initial velocity. The appropriately defined force-time function was used to simulate the effect of a tamper with 10 tones weight dropping from 10 meters high. Two model cases of grid spacing were studied in this investigation: $3 R$ and $2.5 R$, where $R$ is the tamper radius. Two points within the grid were selected to assess the interference between the nearby drops. Based on the observations from the two FE model cases, a grid 
spacing equal to $2.5 \mathrm{R}$ was considered suitable for generating mode uniform treatment effects for the first round of tamping when treating cohesive soils.

Soils were classified according to the parameter C, which is a function of CamClay model parameters, as high or low in terms of suitability for treatment using DC. Instead of requiring the use of cam-clay constitutive model parameters for the design of DC, a simple field test was proposed to classify the soil suitability for DC. Additional manipulations of the derived correlations have resulted in several design charts which can be used to estimate the relative change in soil density as a function of the observed crater depth. Also, a design chart to estimate the PPV based on the value of parameter $\mathrm{C}$ for various tamper mass and falling height was obtained. Finally, a step-by-step design example was presented.

\subsection{Conclusions}

Based on the research work performed, the following conclusions can be drawn.

- ABAQUS FE code could successfully model the initial state of stress exists in the soils.

- Cam-Clay constitutive model was used to describe soil behavior in which the highly non-linear stress-strain behavior of soils could be captured.

- Two 2-D axi-symmetric FE models were developed to model single and multiple drops. To avoid the severe element distortion, a groove with 0.2 meter width and 0.15 meter depth is placed around the peripheral of the tamper for the multiple drop model. 
- The models were validated using actual field measurements for soft clays. Since DC data for sand is more available in the literature, additional FE model was developed to simulate DC in sands. Mohr-coulomb was used. The FE models were considered to be accurate if the results falls in the range of the values obtained from the literature.

- The parametric study conducted using the 2-D models showed that Crater depth $(\mathrm{P})$, void ratio distribution $\left(\Delta e_{o}\right)$, and Peak Particle Velocity (PPV) are sensitive to soil properties as well as tamping energy.

- The propagation of effective zone of influence could be explained using the resulting of the multiple drops 2-D axi-symmetric model.

- Information about soil properties and soil profile before compaction was not provided in the reported data, thus making empirical equations for qualitative estimate.

- Statistically reliable design model was developed for DC in cohesive soils

- The interference between the adjacent spots was investigated using the 3-D FE model by studying the void ratio distribution after compaction of four drops of tamping at each spot.

- Based on the study presented in this chapter, a footprint grid of spacing of $2.5 \mathrm{R}$, where $\mathrm{R}$ is the tamper radius, seems to provide a more uniform treatment than the case of grid spacing of $3 R$.

- If grid spacing of $2.5 \mathrm{R}$ was used, the resulting void ratio variation with depth at Point A (along the sideline of two connecting spots) will be close to those beneath the tamper. 
- Also, If grid spacing of $2.5 \mathrm{R}$ was used, the point at the center of footprint (Point B) will receive the least treatment; approximately $50 \%$ of the treatment beneath the tamper.

- Correlation between density ratio and the induced crater depth was derived based on the correlations presented in chapter IV

- A simple field test was proposed to estimate site specific soil parameter C

- Based on the field measured value of the parameter $\mathrm{C}$, site specific soils can be classified for its suitability for DC

- Design charts for three levels of soil suitability were developed to determine the required crater depth for which the maximum improvement can be achieved down to the desired depth

- Additional design charts were developed to estimate the PPV in correspondence with field measured and site specific value of parameter C.

- The total number of drops for treating a site can be estimated based on the correlation equations presented in this chapter.

- A practical design methodology was developed to allow for a systematic design approach to ensure effective and safe DC operation for a given site soil condition.

- Finally, a step-by-step example was presented to illustrate the appropriate use of the developed design charts and to elucidate the selection process of tamper parameters and $\mathrm{QA} / \mathrm{QC}$ measures 


\subsection{Recommendations for Future Studies}

- More field measurements to validate the proposed design methodology are needed. Collection of detailed information about site conditions and soil properties before compaction will be helpful, since most of the current field measurements are only concerned with the properties after compaction.

- With the increasing computational power, the 2-D simulation work could be replaced by true 3-D simulations.

- Additional investigation should be carried out on the interference between the nearby drops or overlapping drops to allow for more detailed understanding of the interference or interacting mechanisms between the adjacent tamping spots.

- Investigation on the induced pore water pressure in saturated or partially saturated cohesive soils should be a focus of future study, as they will provide more indepth understanding of post-treatment settlement. 


\section{REFERENCES}

1. Abaqus (2007), "Manuals (Benchmark, Analysis and Theory manuals) ", on-line documentation, ABAQUS, Inc, Providence, RI, USA.

2. Das, B. M. (1997). “Advanced Soil Mechanics”, Taylor \& Francis Group, New York.

3. Bai, B. (2006). "Consolidation characteristics and undrained strength of saturated soft clay under repeated impact loading”. Geotechnical Testing Journal; 29(4), Paper ID: GTJ100273.

4. Barat, A. "Study of dynamic compaction method by physical model tests", M.Sc. Thesis, Sharif University of Technology (in Farsi), Tehran, Iran (2001).

5. Bement RAP, Selby AR. (1997) "Compaction of granular soils by uniform vibration equivalent to vibrodriving of piles". Geotech Geolog Engng;15(2):12143. ISSN 0960-3182.

6. Charles, J. A., Burford, D., and Watts, K.S. (1981) " Field studies on the effectiveness of dynamic consolidation", Proceedings of the Tenth International Conference of Soil Mechanics and Foundation Engineering, Stockholm, Sweden, p 617-622

7. Chow, Y.K. Yong, D.M. Yong, K.M., and Lee, S.L. (1994). "Dynamic compaction of loose granular soils effect of print spacing." J Geotech Eng, ASCE. 120(7):1115-33.

8. Chow, Y.K. Yong, D.M. Yong, K.Y., and Lee, S.L. (1992). "Dynamic compaction analysis". Journal of Geotechnical Engineering. 118(8):1141-1157.

9. Chu, J., Yan S.W., and Zheng YR. (2006). "Three soil improvement methods and their applications to road construction". Ground Improvement; 10(3):103-112.

10. Diebels, S. and Ehlers, W. (1996) "Dynamic analysis of fully saturated porous medium accounting for geometrical and material non-linearities". International Journal for Numerical Methods in Engineering; 39:81-97. 
11. Fang, H.Y. and Ellis, G.W. (1983) "Laboratory study of ground response to dynamic densification". Frity Engineering Laboratory Report No. 462-06, Lehigh University, Bethlehem.

12. Fellenius, B. H. and Massarsch, K. R. (2001). "Deep compaction of coarsegrained soils - A case history". A Geotechnical Odyssey: The 54th Annual Canadian Geotechnical Conference. Paper submitted for publication, $8 \mathrm{p}$.

13. Graff, K. F. (1975), "Wave motions in elastic solids", Dover publications, INC., New York, 649 pp.

14. Gu, Q. and Lee, F.H. (2002) "Ground response to dynamic compaction of dry sand". G'eotechnique; 52(7):481-493.

15. Gunaratne, M., Ranganath, M., Thilakasiri, S., Mullins, G., Stinnette, P., and Kuo, C. (1996). "Study of pore pressure induced in laboratory dynamic consolidation". Computers and Geotechnics; 18(2):127-143.

16. Helwany, S. (2007). "Applied Soil Mechanics ABAQUS applications", John Wiley, New York, 400 pp.

17. Terzaghi, K., Peck, R.B., and Mersi, G. (1996). "Soil Mechanics in Engineering Practice", Wiley-Interscience,.

18. Krogh, P. and Lindgren, A. (1997). "Field measurements during deep compaction at Changi Airport", Singapore. Royal Institute Technology (KTH) Stockholm, Sweden;.

19. Lamb, H. (1904). "On the propagation of tremors over the surface of an elastic solid". Philos Trans R Soc Lond 1904;A203:1-42.

20. Lee, F.H., and Gu, Q. (2004) "Method for estimating dynamic compaction effect on sand". Journal of Geotechnical and Geoenvironmental Engineering; 139(2):139-152.

21. Leonards, G.A., Cutter, W.A., and Holtz, R.D. (1981). "Dynamic compaction of granular soils". J Geotech Engng Div, ASCE;106:35-44.

22. Lo, K.W., Ooi, P.L., and Lee, S.L. (1990). "Unfild approach to ground improvement by heavy tamping". J Geotech Eng, ASCE;116(3):514-27.

23. Lopers-Querol, S. Fernandex-Merdod, J.A. Mirap, P., and Pastor, M. (2008). "Numerical modelling of dynamic consolidation on granular soils", Int. J. Numer. Anal. Meth. Geomech.; 32:1431-1457 
24. Lukas, R. G. (1986). "Dynamic compaction of highway construction, design and construction guidlines". Report No. FHWA/RD-86/133, Fedral Highway Administration, Washington D.C

25. Lukas, R.T. (1995). "Dynamic compaction geotechnical engineering circular"., No. 1, Publication no. FHWA-SA-95-037.

26. Budhu, M. (2006). "Soil Mechanics and Foundations", Wiley, New York.

27. M'enard, L. and Broise, Y. (1975) "Theoretical and practical aspects of dynamic consolidation". G'eotechnique; 25(1):3-18.

28. Mayne, P. and Jones, J. (1993) "Impact stresses during dynamic compaction". Journal of Geotechnical Engineering; 109(10):1342-1346.

29. Mayne, P.W., Jones, J.S., and Dumas, J.C. (1984) "Ground response to dynamic compaction". J Geotech Eng, ASCE;110(JT6):757-73.

30. Mayne, P.W. (1985) "Ground vibrations during dynamic compaction. In: Gazetas G, Selig ET", editors. Vibration problems in geotechnical engineering. Special publication of ASCE;p. 247-65.

31. Mcdonal, F. G. and Angona. F. A., Mills, R. L., Sengbush, R. L., VanNostard, R. G., and White, J. E. (1983)"Attenuation of shear and compressional waves in pierre shale" Geophysics, Vol. 23(3), pp. 421-439

32. Mitchell, J.K. and Solymar, Z. V. (1984) "Time-dependent strength gain in fresh deposited or densified sand", ASCE, Journal of Geotechnical Engineering, Vol. 110(11), p 1559-1576.

33. Nashed, R. (2005). "Liquefaction Mitigation of silty soils using dynamic compaction ", Ph.D. Thesis, Department of Civil Engineering, The University of New York at Buffalo.

34. Orrje, O. (1996). "The use of dynamic plate load tests in determining deformation properties of soil". Royal Institute Technology (KTH) Stockholm, Sweden.

35. Oshima, A. and Takada, N. (1994). "Effect of ram momentum on compaction by heavy tamping", International Conference on Soil Mechanics \& Foundation Engineering, 13(3), pp 1141-1144

36. Oshima, A. and Takada, N. (1994). "Relation between compacted area and ram momentum by heavy tamping", International Conference on Soil Mechanics \& Foundation Engineering, 13(4), pp 1641-1194. 
37. Oshimam, A. and Takada, N. (1994). "Comparison between field and centrifuge model test of heavy tamping. In: Proceedings of the centrifuge", Singapore; p. $337-42$.

38. Oshimam, A. and Takada, N. (1998) "Evaluation compacted area of heavy damping by cone point resistance. In: Proceedings of the centrifuge", Tokyo; $p$. $813-8$.

39. Pan, J. and Hwang, Z.M. (1995). "Soil densification by dynamic consolidation for Formosa heavy industries corp". Sino Geotech;51:35-50.

40. Pan, J.L and Selby A.R. (2002) "Simulation of dynamic compaction of loose granular soils". Advances in Engineering Software; 33:631-640.

41. Perucho, A. and Olalla, C. (2006) "Dynamic consolidation of a saturated plastic clayey fill". Ground Improvement; 10(2):55-68.

42. Poran, C.J. and Rodr'1guez J.A. (1992) "Design of dynamic compaction". Canadian Geotechnical Journal; 29:796-802.

43. Poran, C.J., Heh, K.S. and Rodriguez, J.A. (1992)."Impact behavior of sand", Soils and Foundations, 32(4), pp 81-92

44. Richart, F. E., Hall, J. R., and Woods, R. D. (1970) "Vibrations of soils and foundations", Prentice-Hall, Englewood Cliffs, NJ.

45. Robertson, P.K. and Campanella, R.E. (1983). "Interpretation of cone penetration tests". Can Geotech J;20(4):718-33.

46. Roesset, E., Kausel, J.M., Cu'ellar, V., Monte, J.L., and Valerio, J. (1994). "Impact weight falling onto the ground". Journal of Geotechnical Engineering; 120(8):1394-1412.

47. Rollins, K. M. and Kim, J. H. (1994) "U.S. experience with dynamic compaction of collapsible soils'. In-situ deep soil improvement, ASCE, Geotechnical special publication, No. 45,p 2+6-43

48. Lee, S.R. and Kim, Y.S. (2000). "Analysis of sedimentation/consolidation by finite element method", Computers and Geotechnics 27 (2) 141-160

49. Scott, A. and Pearce, W. (1975). "Soil compaction by impact". G'eotechnique; 25(1):19-30. 
50. Skipp, B. O. and Barkley, J. S. (1977). "Ground vibration from impact. In: Proceedings of the ninth international conference on soil mechanics and foundation engineering", Tokyo, Japan, vol. 2; p. 397-401.

51. Slocombe, B. C. (1993) "Dynamic compaction. In" Chapter 2 in Ground improvement, Moseley, M. P., Editor. CRC Press, Florida

52. Smits, M. Th.J.H, and De Quelerij, L. (1989). " the effect of dynamic compaction on dry granular soils". Proceedings, $12^{\text {th }}$ international Conference of Soil Mechanics and Fundations Enginering, Rio de Janeiro, vol. 2, pp. 1419-1422

53. Thilakasiri, H. S., Gunaratne, M., Mullins, G., Stinnette, P., and Jory, B. (1996). "Investigation of impact stresses induced in laboratory dynamic compaction on soft soils". International Journal for Numerical and Analytical Methods in Geomechanics; 20:753-767.

54. West, J. M., Slocombe, B.C. (1973). "Dynamic consolidation as an alternative foundation". Ground Engng; 6(6):52-4.

55. woods, R. D., and Jedele, L. P. (1985) "Energy attenuation relationships from construction vibration", Vibration problems in geotechnical engineering, Gazetas and selig, editors, ASCE , pp. 247-265

56. Zerwer, A., Cascante, G., and Hutchinson, J. (2002), "Parameter Estimation in Finite Element Simulations of Rayleigh Waves", ASCE, Journal of Geotechnical and Geoenvironmental Engineering, 250-261 pp.

57. Zienkiewicz, O. C., Chang, C.T., and Bettes, P. (1980). "Drained, undrained, consolidating and dynamic behaviour assumptions in soils". G'eotechnique; 30(4):385-395.

58. Zienkiewicz, O. C., Emson, C., Bettess, P. (1983) "A novel boundary infinite element". Int J Numer Meth Engng; 19:393-404. 